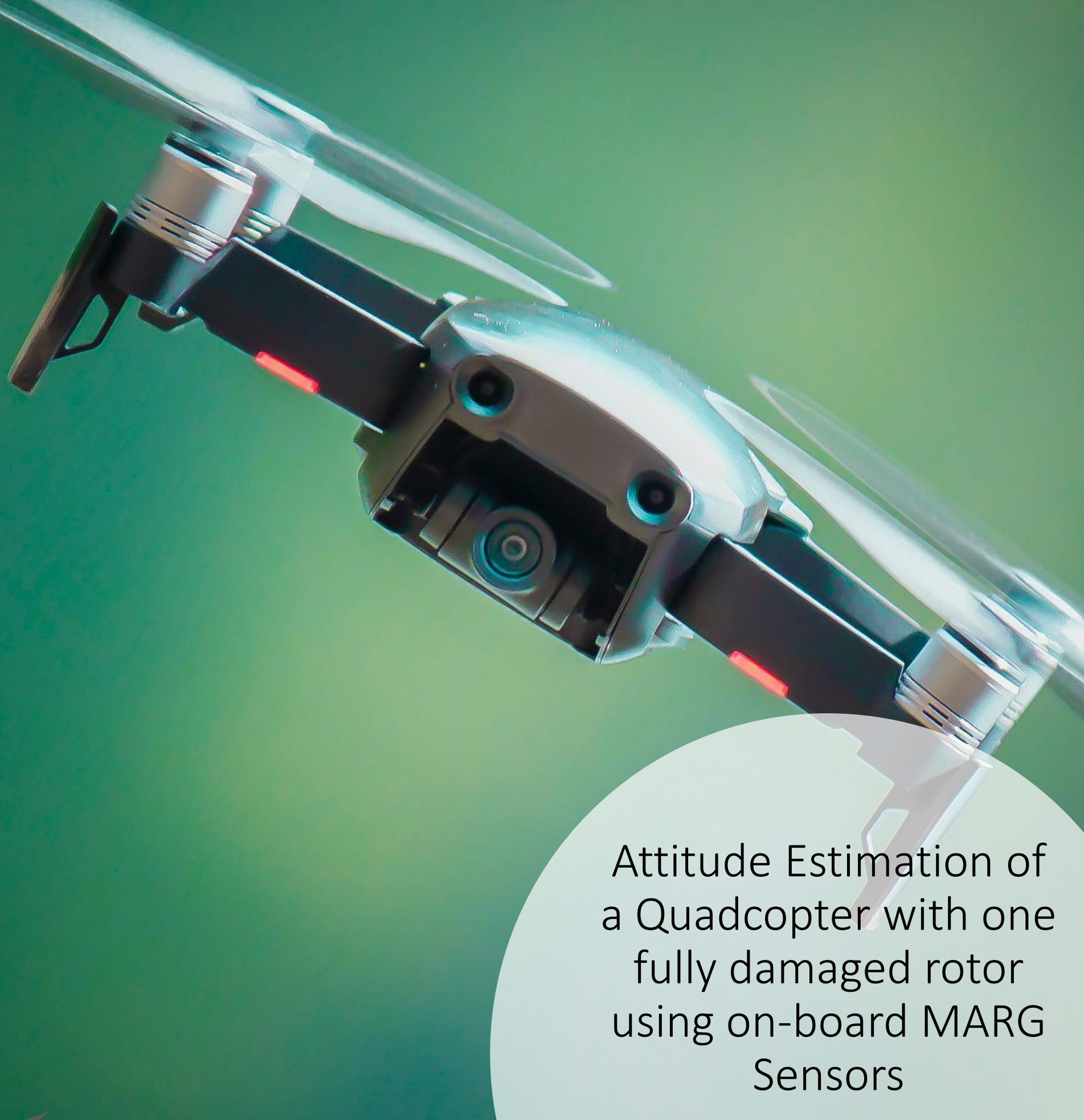

Prashant Solanki 



\title{
Attitude Estimation of a Quadcopter with one fully damaged rotor using on-board MARG Sensors
}

\author{
Submitted by \\ Prashant Solanki (4794761)
}

in partial fulfilment of the requirements for obtaining the

degree of

MASTER OF SCIENCE IN AEROSPACE ENGINEERING

Supervisors:

Dr ir C. C. de Visser

Sihao Sun

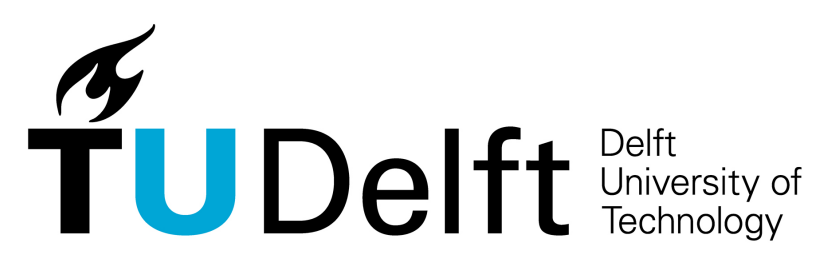





\section{Acknowledgements}

I would like to acknowledge my thesis supervisors Dr ir C.C. de Visser and Sihao Sun. This thesis was only possible because of their immense support and extremely helpful guidance. Their insight and expertise were crucial for this research. From the very fist step of the literature review to the final step of validating the filter, their supportive nature made this journey enjoyable. Furthermore, I am very grateful to my parents, my partner Josephine, and my brother for their constant support and multiple rounds of proofreading. As the famous Indian saying goes, "One can achieve anything if surrounded by the right people" - and I believe that I have found the right people. 



\section{Summary}

Quadcopters are becoming increasingly popular across diverse sectors such as mapping, photography, or surveillance. Since rotor damages occur frequently, it is essential to improve the attitude estimation and thus ultimately the ability to control a damaged quadcopter. The Control and Simulation group of TU Delft developed a quadcopter controller for the case of a single damaged rotor in an inside environment, where the attitude and position of the quadcopter are provided by an external system. In the present research, a novel attitude estimator called Adaptive Fuzzy Complementary Kalman Filter (AFCKF) has been developed and validated that works independently of any external systems. It is able to estimate the attitude of a quadcopter with one fully damaged rotor while only relying on the on-board sensors. The results show that the AFCKF is able to provide significantly better attitude estimates for flights with a damaged rotor than mainstream filters, estimating the roll and pitch of the quadcopter with an RMS error of less than 1.7 degrees and a variance of less than 2 degrees. It is the only filter that is able to track the quadcopter's yaw angle for the case of a damaged rotor, while showing only a comparatively small rise in the computational cost. 


\section{Contents}

Summary

List of Figures $\quad$ v

List of Algorithms vi

List of Tables vii

List of Acronyms viii

I Preliminary Report 1

1 Introduction $\quad 2$

2 An overview of sensors $\quad 4$

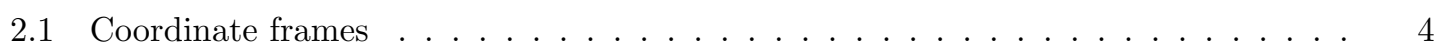

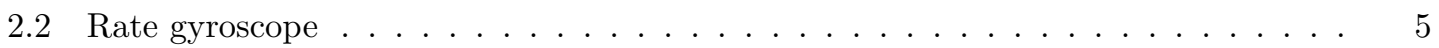

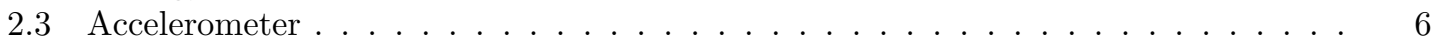

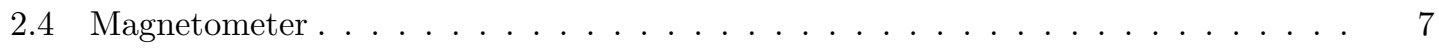

3 Attitude parameterization schemes $\quad 8$

3.1 Direction cosine matrix $\ldots \ldots \ldots \ldots \ldots \ldots$

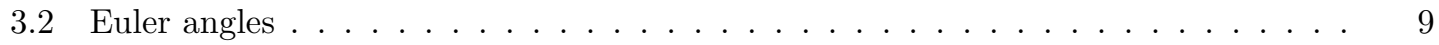

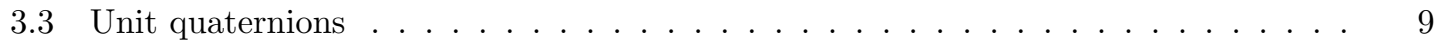

3.4 Discussion . . . . . . . . . . . . . . . . . . . . . . . 11

4 The mathematical model of a quadcopter 12

4.1 A quadcopter's kinematics model . . . . . . . . . . . . . . . . . . . . . . . . . 12

4.2 A quadcopter's dynamic model . . . . . . . . . . . . . . . . . . . . . . . . . . . 12

4.3 Measurement Model . . . . . . . . . . . . . . . . . . . . . . . . . . . . 15

5 A classification of state estimation methods $\quad \mathbf{1 7}$

5.1 Data driven-based estimation . . . . . . . . . . . . . . . . . . . . . . . . . 17

5.2 Vehicle model-based estimation . . . . . . . . . . . . . . . . . . 18

5.3 Dynamic-model based method . . . . . . . . . . . . . . . . . . . . . . . 18

5.4 Observer-based methods . . . . . . . . . . . . . . . . . . . . . . . . . 19

6 Deterministic Approach 21

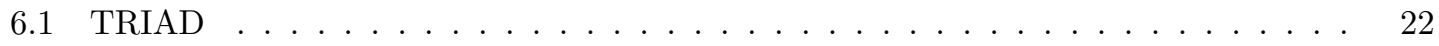

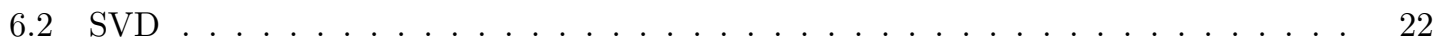

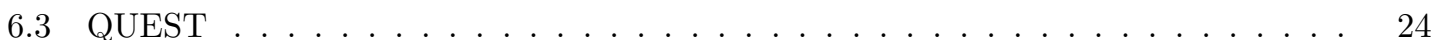

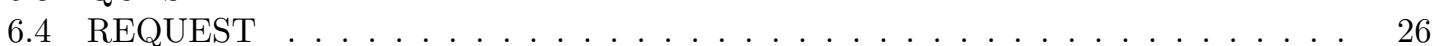

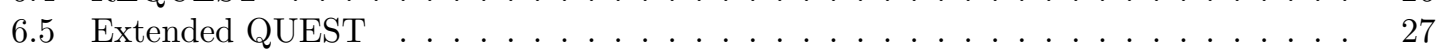

6.6 Discussion . . . . . . . . . . . . . . . . . . . . . . . . . . . . 29

7 Complementary filters $\quad 30$

7.1 Mahony Filter . . . . . . . . . . . . . . . . . . . . . . . . . 31

7.2 Madgwick filter . . . . . . . . . . . . . . . . . . . . . . . . . . . . 33

7.3 Adaptive-gain complementary filter . . . . . . . . . . . . . . . . . . 38

7.4 Geometrically intuitive complementary filter . . . . . . . . . . . . . . . . 39

7.5 Fast complementary filter . . . . . . . . . . . . . . . . . . . . . . 42

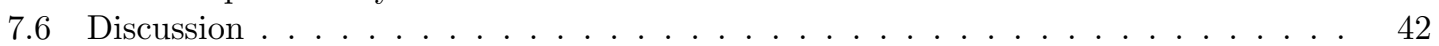


8 Stochastic Estimation $\quad 43$

8.1 Bayesian Filters . . . . . . . . . . . . . . . . . . . . . . . 43

8.2 Extended Kalman Filter . . . . . . . . . . . . . . . . . . . . . . . . . . . . . . . . 45

8.3 Unscented Kalman Filter . . . . . . . . . . . . . . . . . . . . . . . . . . . . . 47 47

8.4 Attitude estimation . . . . . . . . . . . . . . . . . . . 50

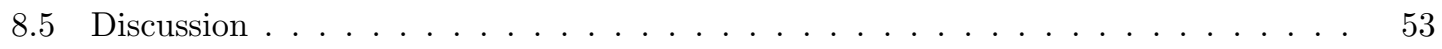

9 Conclusions $\quad \mathbf{5 4}$

9.1 Preliminary Filter Design . . . . . . . . . . . . . . . . . . . . . 55

9.2 Research Plan Summary . . . . . . . . . . . . . . . . . . . . . 58

$\begin{array}{lr}\text { References } & 60\end{array}$

II Scientific Paper $\quad 66$ 


\section{List of Figures}

2.1 Pictorial representation of the frames of reference . . . . . . . . . . . . . 5

5.1 Classification of vehicle attitude estimation methodologies . . . . . . . . . . . 17

5.2 The schematic of ANN estimation process . . . . . . . . . . . . . . 18

7.1 The schematic of the complementary filter by Baerveldt and Klang (1997) . . . . . 30

7.2 Realisation of a complementary filter . . . . . . . . . . . . . . . . . 31

7.3 Mahony filter Schematics . . . . . . . . . . . . . . . . . . . . . 33

7.4 Madgwick filter Schematics (Madgwick et al. (2011)) . . . . . . . . . . . . . . . 35

7.5 Filter schematics (Tian et al. (2013)) . . . . . . . . . . . . . . . . . 38

7.6 Adaptive-gain complementary filter schematics (Calusdian et al. (2011)) . . . . . . 38

7.7 Adaptive filter by Marantos et al. (2016) . . . . . . . . . . . . . . . . . . 39

7.8 Geometrically intuitive approach complementary filter schematics (Del Rosario et al.

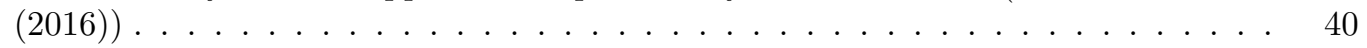

7.9 Fast complementary filter schematics (Wu et al. (2016)) . . . . . . . . . . . . 42

8.1 Kalman filter illustration . . . . . . . . . . . . . . . . . . 46

8.2 illustration of the difference between EKF and UKF . . . . . . . . . . . . . . . . . 49

8.3 The schematic of EFK in Marins et al. (2001): Method 1 . . . . . . . . . . . 50

8.4 The schematic of Kalman filter in Marins et al. (2001): Method 2 . . . . . . . . . 50

8.5 The schematics of the Kalman filter in Xiaoping et al. (2005) . . . . . . . . . . . . 51

8.6 The schematic of EFK in the paper Sabatini (2006) . . . . . . . . . . . . . 51

8.7 The schematic filter presented by De Marina et al. (2012) . . . . . . . . . . . . . 52

8.8 The schematic filter presented in the paper Yuan et al. (2015) . . . . . . . . . 53

9.1 Schematics of current proposed filter . . . . . . . . . . . . . . . . 56 


\section{List of Algorithms}

1 Mahony filter algorithm . . . . . . . . . . . . . . . . . . . . . . . 32

2 Madgwick filter algorithm . . . . . . . . . . . . . . . . . . . . . 36

3 Geometrically intuitive complementary filter algorithm . . . . . . . . . . . . 41

4 Linear Kalman filter algorithm . . . . . . . . . . . . . . . . . . . . 45

5 Extended Kalman filter algorithm _. . . . . . . . . . . . . . . . . 47

6 Unscented Kalman filter algorithm . . . . . . . . . . . . . . . . . . . . . . 48 


\section{List of Tables}

4.1 Estimated values for the parameters (Sun et al. (2018a)) . . . . . . . . . . . . . 14

4.2 Estimated values for the parameters (Sun et al. (2018a)) . . . . . . . . . . . . . 14

4.3 Estimated values for the parameters (Sun et al. (2018a)) . . . . . . . . . . . 15

4.4 Estimated values for the parameters (Sun et al. (2018a)) . . . . . . . . . . . 15

7.1 Performance comparison . . . . . . . . . . . . . . . . . . 37

7.2 Computational cost comparison . . . . . . . . . . . . . . . . 37 


\section{List of Acronyms}

AEKF - Additive Extended Kalman Filter

AFCKF - Adaptive Fuzzy Complementary Kalman Filter

ANFIS - Adaptive neuro fuzzy inference system

DCM - Direction Cosine Matrix

EKF - Extended Kalman Filter

ESOQ - Estimation of Optimal Quaternion

FOAM - Fast Optimal Attitude Matrix

GRNN - General Regression Neural Network

IEKF - Iterated Extended Kalman Filter

INS - Inertial Navigation System

IUKF - Iterated Unscented Kalman Filter

LMI - Linear Matrix Inequalities

MARG - Magnetometer, Accelerometer, and Rate Gyroscope

MEKF - Multiplicative Extended Kalman Filter

MEMS - Microelectromechanical systems

MMF - Multiple Model Filter

NLO - Nonlinear Observer

QUEST - Quaternion Estimation

REQUEST - Recursive Quaternion Estimation

SMO - Sliding Mode Observers

SOAR - Sequential Optimal Attitude Recursion

SVD - Singular Value Decomposition

UIO - Unknown Input Observer

UKF - Unscented Kalman Filter 
Part I

Preliminary Report 


\section{Introduction}

By virtue of mechanical simplicity, quadcopters have become very popular in multiple industries such as delivery, surveillance, photography, mapping, and more. Although cheap and versatile in nature, the quadcopter suffers from the inherent problem of a lack of redundancy in the control surface (rotors). A wide range of research has been conducted to develop a fault-tolerant controller for a quadcopter subjected to rotor failure (Mueller and D'Andrea (2014), Mueller and D'Andrea (2016), Sun et al. (2018b)). In the scenario of a complete single rotor failure, the quadcopter has to abandon yaw control and spin at a high angular rate. This motion improves the stability of the system due to gyroscopic effect, but deteriorates the position and attitude estimation.

The C\&S group of TU Delft has managed to develop a fault-tolerant controller and successfully conduct an indoor flight of a quadcopter subjected to a complete single rotor failure (Sun et al. (2018b)). The attitude of the quadcopter was attained using a motion-capture system that provides the system's/vehicle's attitude in order to test the controller. Unfortunately, no such motion capture system exists for the outside environment. Thus, an attitude estimation method is required to be developed that can estimate the states of the quadcopter with sufficient accuracy using only the on-board sensors (magnetometer, rate gyroscope, and accelerometer). This is the stated aim of this present research. However, these on-board sensors have a variety of problems that makes the task of attitude estimation more complicated.

The accelerometer measures the specific force that is applied to the sensor. In an ideal situation, the accelerometer is situated at the center of mass of the quadcopter, but in reality it is not possible to place the accelerometer directly at the center of mass. Furthermore, the sensor suffers from high noise and time-varying bias issues. The rate gyroscope measures the rotation velocity of the sensor, which is the same as the quadcopter angular velocity under the rigid body assumption, but, just as the accelerometer, the rate gyroscope readings have a lot of noise and are also effected by time-varying bias. The magnetometer measures the local magnetic field. In an ideal situation, the local magnetic field is the same as the earth's local magnetic field, which is known, but in reality the measured reading is corrupted with local magnetic disturbance, noise and time-varying bias.

The main research objective of this thesis is:

"To develop an attitude estimation method that can estimate the states of a quadcopter with one fully damaged rotor with sufficient accuracy, while only using the on-board sensors (magnetometer, rate gyroscope, and accelerometer)".

In order to address the problems of each sensor and develop an attitude estimation technique, the following sub-research questions need to be addressed:

- What are the sources of errors and shortcomings of the magnetometer, rate gyroscope, and accelerometer?

- Which are the most popular attitude estimation methods in the field of online quadcopter attitude estimation?

- Are any of these techniques suitable for the three-dimensional attitude estimation of a quadcopter in a high-speed yaw rate spinning condition while using only on-board sensors (accelerometer, gyroscope, magnetometer)?

- If not, which shortcomings prevent these techniques from being suitable for estimating the states of a quadcopter in a high-speed yaw rate spinning condition while only using the listed on-board sensors?

- How can these shortcomings be overcome by developing a new attitude estimator that can estimate the states of a quadcopter in a high-speed yaw rate spinning condition while only using the listed on-board sensors? 
To develop a satisfactory attitude estimation algorithm, this preliminary thesis report surveys the existing literature to find the most suitable method. The second chapter provides an overview over the on-board sensors, their respective mathematical model, and their issues. The third chapter provides an overview and a discussion of the different parameterization schemes of attitude representation that are most widely used in the aerospace industry. It addresses their drawbacks and provides reasons for choosing one particular parameterization scheme. In the fourth chapter, two quadcopter mathematical models are discussed, namely the reduced model and the complex model (Sun et al. (2018a)). Chapter 5 introduces a classification of the available state estimation methods. After briefly discussing the less suitable methods in the second part of chapter 5 , chapter 6,7 , and 8 concentrate on discussing the more promising approaches.

Chapter 6 discusses the deterministic approach, which uses mainly single point algorithms, i.e., algorithms that determine the attitude of the system at the point of time when the observation of a known vector (inertial frame) is available in the body reference frame without considering any past information or observation. After that, chapter 7 surveys the complementary filter approach, which works by combining the readings of multiple complementary sensors to obtain a better attitude estimate. Chapter 8 gives an overview of stochastic estimation methods and its most prominent methods, i.e., the Kalman filter and its variants. Chapter 9 comes to the conclusion that none of the methods are directly applicable for the stated aim of the research. Thus, in the final chapter, a preliminary filter design will be provided that draws on a mixture of the best-suited filters from the previous literature study. 


\section{An overview of sensors}

\section{Introduction}

This chapter focuses on the sensors that are available on board of the quadcopter. The three sensors that will be used for attitude estimation of the quadcopter will be discussed in-depth, as well as their sensor measurement models and drawbacks. The chapter starts by introducing different reference frames that are used for the development of the measurement model, followed by a detailed explanation of the measurement model of the sensors.

The available on-board sensors that will be discussed are:

- Rate gyroscope

- Accelerometer

- Magnetometer

To understand the workings and shortcomings of these sensors, firstly a discussion of multiple coordinate frames is necessary.

\subsection{Coordinate frames}

In order to discuss the data measured by the sensors, mainly accelerometer, gyroscope and magnetometer, four coordinate frames are required to be introduced. Figure 2.1 shows the pictorial representation of the reference frames.

\section{The body frame $B$}

The first frame is called the body frame. The body frame is fixed to the system's body and moves along with it. All measurements taken are taken inside the body frame. Usually, the accelerometer and the gyroscope are situated on the origin of the body frame, which is where most calculations assume them to be. However, this assumption may prove to be problematic, as will be explained later. In respect to the current report, the body reference frame is assumed to be a NED frame (North, East and Down). If the $x$-axis of the body frame is pointing towards north, then the $y$-axis is pointing towards east and is perpendicular to the $x$-axis. The $z$-axis is pointing downwards and is perpendicular to the $x$ and $y$-axis. The unit vector of the body frame is represented as $\hat{E}_{B}$ and a vector in the body frame is depicted via subscript $B$ as $\vec{A}_{B}$ is a vector in body frame.

\section{The navigation frame $N$}

The second frame is called the navigation frame, which gives us the position and orientation of the body frame respective to the navigation frame. Typically, the navigation frame is defined stationary with respect to the earth, but if the body is traveling over a long distance, then the navigation frame also movies along the surface of the earth. For the current paper it is assumed that the quadcopter is flying locally and the navigation frame can be assumed to be stationary over the earth surface. This implies that the navigation frame and inertial frame can be used interchangeably. The unit vector in the navigation frame is depicted by $\hat{E}_{N}$ and a vector in the navigation frame is represented with a subscript $N$ as $\vec{A}_{N}$.

\section{The inertial frame $I$}

The third frame is called the inertial frame. This frame is stationary, which means that it does not move. The measurements made by the accelerometer and the gyroscope are with respect to the inertial frame, i.e., the linear acceleration measured by the accelerometer and the angular velocity measured by the rate gyroscope are made with respect to the inertial frame of reference. The centre of the earth constitutes the inertial frame's origin and its axes are being aligned with the stars. The unit vector in the inertial frame is depicted by $\hat{E}_{I}$ and a vector in the inertial frame is represented with a subscript $I$ as $\vec{A}_{I}$. 


\section{The earth frame $E$}

The last coordinate frame is the earth frame. Different from the inertial frame, the earth frame moves along with the earth's rotation. Its origin is also constituted by the centre of the earth, but its axes are fixed respective to the earth. For short flight times (less than an hour), the inertial frame and the earth reference frame can be used interchangeably. The unit vector in the earth frame is depicted by $\hat{E}_{E}$ and a vector in the earth frame is represented with a subscript $I$ as $\vec{A}_{E}$.

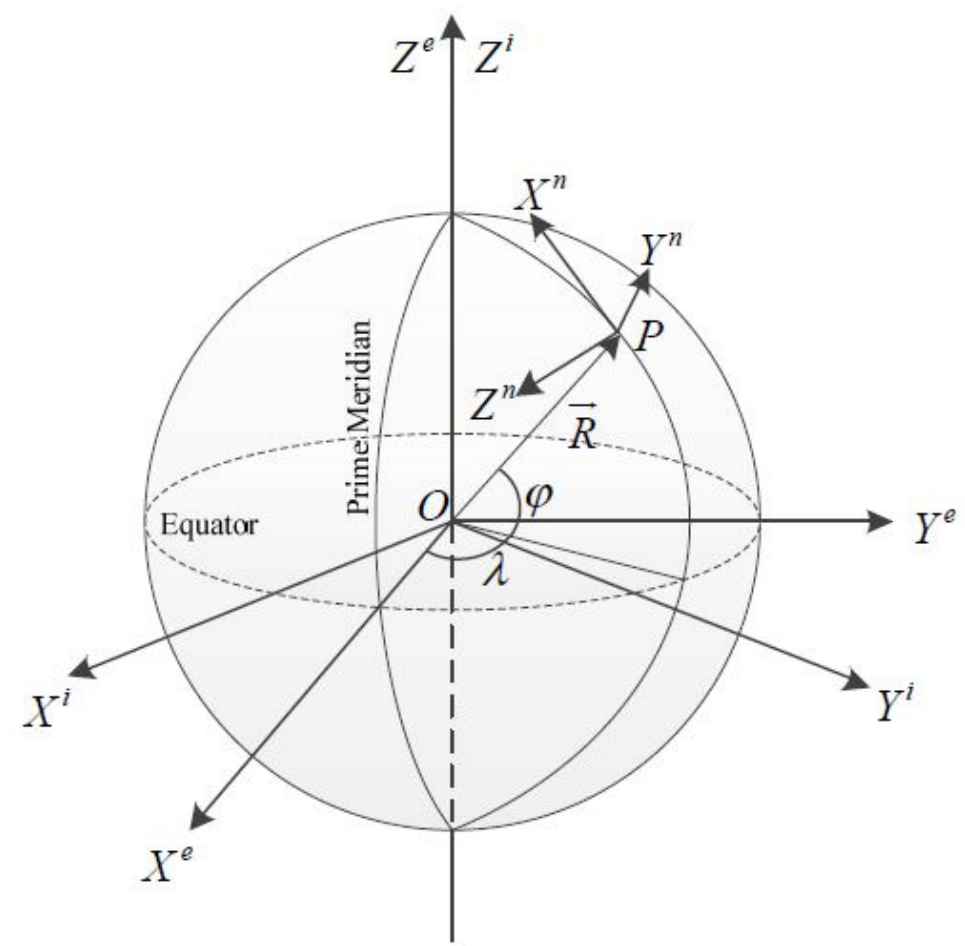

Figure 2.1: Pictorial representation of the frames of reference

\subsection{Rate gyroscope}

The rate gyroscope measures the quadcopter's angular rate (angular velocity) with respect to the inertial frame represented in the body frame of reference. Let the angular rate be denoted as $\omega_{B I}$ or $\omega_{B}$. The relationship of the angular velocity in different frames is given by equation 1 , where $\omega_{E I}$ is the angular velocity of the earth with respect to the inertial frame, which is equal to $7.29 \cdot 10^{-5} \mathrm{rad} / \mathrm{sec}$ (Williams $(2020)$ ). $\omega_{N E}$ is the angular velocity of the navigation frame with respect to the earth frame which is equal to zero (due to assumption 2.1), $\omega_{B N}$ is the angular velocity of the quadcopter with respect to the navigation frame, and $R_{B / N}$ is the rotation matrix from navigation frame to body frame of reference.

$$
\omega_{B I}=R_{B / N}\left(\omega_{E I}+\omega_{E N}\right)+\omega_{B N}
$$

The angular velocity of the earth with respect to the inertial frame is negligible with respect to the angular velocity of a quadcopter, i.e., $\omega_{E I}=0$. Equation 1 can be approximated to equation 2

$$
\omega_{B I} \approx \omega_{B N}
$$

MEMS gyroscopes that are used in quadcopters are not very high-quality rate gyroscopes, and their readings are corrupted with random noise and a bias which is variable and changes with time. The readings of the MEMS rate gyroscope are given in equation 3 , where $e_{\omega}$ is the random 
noise of the sensor and $\delta_{\omega, t}$ is the time-varying bias of the rate gyro. It is assumed that the random noise of the rate gyro $\left(e_{\omega}\right)$ is Gaussian white noise with a zero mean and having a standard deviation $\Sigma_{\omega}$. As it can be seen from the definition of the $\Sigma_{\omega}$, it is assumed that there is no correlation between the three axes.

$$
\begin{gathered}
\Sigma_{\omega}=\left(\begin{array}{ccc}
\sigma_{\omega, x} & 0 & 0 \\
0 & \sigma_{\omega, y} & 0 \\
0 & 0 & \sigma_{\omega, z}
\end{array}\right) \\
y_{\omega}=\omega_{B I}+e_{\omega}+\delta_{\omega, t}
\end{gathered}
$$

From equation 3 and 2

$$
y_{\omega}=\omega_{B N}+e_{\omega}+\delta_{\omega, t}
$$

Consequently, in regards to this research, it is assumed that the rate gyroscope measures the quadcopter angular velocity with respect to the inertial/navigation frame along with bias and noise.

\subsection{Accelerometer}

The accelerometer is also an inertial sensor and it measures the specific force in the body fame of reference with respect to the inertial frame, which can be expressed as equation 5 . Here $f_{B}$ is the specific force, $a_{I}$ is the linear acceleration of the quadcopter in the navigation frame, $g_{N}$ is the gravity vector in the navigation frame and $R_{B / N}$ is the rotation matrix from navigation to the body frame of reference.

$$
\begin{gathered}
\overrightarrow{f_{b}}=R_{B / N}\left(\vec{a}_{N}-\vec{g}_{N}\right) \\
\vec{a}_{N}=R_{N / E} R_{E / I} \vec{a}_{I}
\end{gathered}
$$

Due to assumption 2.1 , the $\vec{a}_{N}$ can be derived as equation 6 , where $\omega_{A / B}$ is the angular velocity of frame $B$ with respect to frame $A$ expressed in frame $A$.

$$
\vec{a}_{I}=\vec{a}_{N}+\vec{\omega}_{N / I} \times \vec{\omega}_{N / I} \times \vec{p}_{N}+2 \vec{\omega}_{N / I} \times \vec{v}_{N}
$$

For navigation purposes, $\vec{a}_{N}$ is the required acceleration. The terms $2 \vec{\omega}_{N / I} \times \vec{v}_{N}$ and $\vec{\omega}_{N / I} \times \vec{\omega}_{N / I} \times$ $\vec{p}_{N}$ represent Coriolis acceleration and centrifugal acceleration respectively. The centrifugal acceleration is absorbed by the gravity vector and Coriolis acceleration is very small value compared to the gravity vector, and thus it can be neglected.

$$
\vec{a}_{I} \approx \vec{a}_{N}
$$

Similar to the rate gyroscope, the accelerometer used in IMU are of poor quality and are plagued by random noise and time-varying bias. Thus an accelerometer reading can be described by equation 8 .

$$
y_{a}=R_{B / N}\left(\vec{a}_{N}-\vec{g}_{N}\right)+e_{a}+\delta_{a, t}
$$

where $e_{a}$ is the random noise of the sensor and $\delta_{a, t}$ is the time varying bias of the accelerometer. It is assumed that the random noise of the accelerometer $\left(e_{a}\right)$ is Gaussian white noise with zero mean and having a standard deviation $\Sigma_{a}$. As it can be seen from the definition of $\Sigma_{a}$, it is assumed that there is no correlation between the three axis. 


$$
\Sigma_{a}=\left(\begin{array}{ccc}
\sigma_{a, x} & 0 & 0 \\
0 & \sigma_{a, y} & 0 \\
0 & 0 & \sigma_{a, z}
\end{array}\right)
$$

\subsection{Magnetometer}

The magnetometer measures the local magnetic field, which consists of both the local earth's magnetic field and the magnetic field due to any nearby magnetic surface. The ratio of the horizontal to the vertical magnetic field is expressed in terms of the local dip angle denoted by $\delta$. Geophysical studies provide an accurate data of the earth's magnetic field around the earth (NCEI (2020)) Due to assumption 2.1 we can model the earth's magnetic field as a constant. The model (a constant vector in our case) is given in equation 9, where $|m|$ is the local earth's magnetic field strength and $\vec{m}_{N}$ is the earth's magnetic field vector in the navigation frame.

$$
\vec{m}_{N}=|m|\left[\begin{array}{lll}
\cos \delta & 0 & -\sin \delta
\end{array}\right]_{N}^{t}
$$

The earth's magnetic field can also be modeled in terms of the vertical component of earth's magnetic field, as shown in equation 10 .

$$
\vec{m}_{N}=\frac{1}{|m|}\left[\sqrt{1-m_{N_{Z}}^{2}} \quad 0 \quad m_{N_{z}}\right]^{T}
$$

Similar to the previous sensors, the magnetometer also has noise and a time-varying bias. Thus a magnetometer reading can be described by equation 11 .

$$
y_{m}=R_{b n}\left(m_{n}\right)+e_{m}+\delta_{m, t}
$$

where $e_{m}$ is the random noise of the sensor and $\delta_{m, t}$ is the time-varying bias of the magnetometer. It is assumed that the random noise of the magnetometer $\left(e_{m}\right)$ is Gaussian white noise with zero mean and having a standard deviation $\Sigma_{m}$. As can be seen from the definition of $\Sigma_{m}$, it is assumed that there is no correlation between the three axis.

$$
\Sigma_{a}=\left(\begin{array}{ccc}
\sigma_{m, x} & 0 & 0 \\
0 & \sigma_{m, y} & 0 \\
0 & 0 & \sigma_{m z}
\end{array}\right)
$$




\section{Attitude parameterization schemes}

\section{Introduction}

In order to develop an attitude filter for a quadcopter, its orientation with respect to a certain frame needs to be known. The attitude or orientation of a system can be represented in multiple ways. There are many different parameterization schemes available, such as Euler angles, quaternions, Rodrigues parameters, modified Rodrigues parameters, etc. All the different schemes have their own benefits and drawbacks. which will be discussed in this chapter. In the end, a conclusion will be drawn about which scheme is the most suitable for an application on the quadcopter. Stuelpnagel (1964), Shuster (1993) and Hughes (2012) provide a more detailed insight into various ways of representing attitude. Among all the available representation schemes the following schemes are the major attitude representation schemes used in the the field of aerospace:

- Direction cosine matrix

- Euler angles

- Quaternions

\subsection{Direction cosine matrix}

The direction cosine matrix is one of the most basic orientation representation schemes. If there are two orthogonal frames of reference, $A$ and $B$, defined by orthogonal unit vectors $\vec{a}_{1}, \vec{a}_{2}, \vec{a}_{3}$ and $\vec{b}_{1}, \vec{b}_{2}, \vec{b}_{3}$ (both the frames need to be either right hand or left hand), then one frame of reference can be represented in terms of the other as shown in equation 12 . Here, $C_{i j}$ is equal to cosine of the angle in between $\vec{a}_{i}$ and $\vec{b}_{j}$, given by $\vec{a}_{i} \cdot \vec{b}_{j}$.

$$
\begin{aligned}
& \vec{b}_{1}=C_{11} \vec{a}_{1}+C_{12} \vec{a}_{2}+C_{13} \vec{a}_{3} \\
& \vec{b}_{2}=C_{21} \vec{a}_{1}+C_{22} \vec{a}_{2}+C_{23} \vec{a}_{3} \\
& \vec{b}_{3}=C_{31} \vec{a}_{1}+C_{32} \vec{a}_{2}+C_{33} \vec{a}_{3}
\end{aligned}
$$

The above equation can be written as

$$
\begin{gathered}
{\left[\begin{array}{l}
\vec{b}_{1} \\
\vec{b}_{2} \\
\vec{b}_{3}
\end{array}\right]=C^{A / B}\left[\begin{array}{l}
\vec{a}_{1} \\
\vec{a}_{2} \\
\vec{a}_{3}
\end{array}\right]} \\
C^{A / B}=\left[\begin{array}{lll}
C_{11} & C_{12} & C_{13} \\
C_{21} & C_{22} & C_{23} \\
C_{31} & C_{32} & C_{33}
\end{array}\right]
\end{gathered}
$$

where $C^{A / B}$ is the rotation matrix and $I$ is the identity matrix (3x3). Such rotation matrices have the properties defined by equation 13 . These matrices are called special orthogonal group $S O(3)$.

$$
\begin{gathered}
C^{A / B} * C^{B / A}=I \\
\left|C^{A / B}\right|=1
\end{gathered}
$$

If the rotation matrix between frame $A$ and $B$ is known, a vector of one frame can be converted into the other frame using equation 14.

$$
\vec{y}^{A}=C^{A / B} \vec{y}^{B}
$$




\subsection{Euler angles}

Euler angle is the attitude representation scheme in which one frame can be transformed into the desired frame by three consecutive rotations about three orthogonal axes of the first frame. The first rotation can be about any of the three axes, the second rotation can be made about any two axes not used in first rotation, and the final rotation can be made about any two axes not used in the second rotation. This allows a total of twelve different ways in which one reference frame can be transformed into the desired frame (both the frames must follow either right or left hand notation). Euler angles are among the most widely used attitude representation schemes. This is due to the fact that the scheme is easy to understand and is intuitive in nature but it sufferers from singularity issues. The singularity issue is the common problem for all three-dimension representation schemes. Furthermore it is base on calculation if sine and cosine function which are often approximated, this approximation leads to approximation errors.

Equation 15 gives the rotation matrix obtained via 2-3-1 rotation.

$$
\begin{gathered}
C^{B / A} \equiv C_{1}\left(\theta_{1}\right) C_{3}\left(\theta_{3}\right) C_{2}\left(\theta_{2}\right)=\left[\begin{array}{ccc}
c_{2} c_{3} & s_{3} & -s_{2} c_{3} \\
-c_{1} c_{2} s_{3}+s_{1} s_{2} & c_{1} c_{3} & c_{1} s_{2} s_{3}+s_{1} c_{2} \\
s_{1} c_{2} s_{3}+c_{1} s_{2} & -s_{1} c_{3} & -s_{1} s_{2} s_{3}+c_{1} c_{2}
\end{array}\right] \\
\text { where: } s_{1}=\sin \left(\theta_{1}\right), s_{2}=\sin \left(\theta_{2}\right), s_{3}=\sin \left(\theta_{3}\right), c_{1}=\cos \left(\theta_{1}\right), c_{2}=\cos \left(\theta_{2}\right), c_{3}=\cos \left(\theta_{3}\right)
\end{gathered}
$$

If $C^{A / B}$ is known, then a vector in frame $A$ can be converted to a frame $B$ vector using equation 14.

\subsection{Unit quaternions}

A quaternion is a four-dimensional complex number. There are multiple representaion schemes with a such as Cayley-Klein parameters and the Rodrigues parameters that have a redundant member. The survey by Shuster (1993) provide the their connection to each other and definition of such representation schemes. Quaternion are used to describe the orientation of a frame with respect to another frame. A reference frame $A$ can be rotated about an axis (unit axis) in frame $A\left(r^{A}\right)$ by an angle theta $(\theta)$ to transform the frame $A$ into frame $B$, provided both the frames follow either left hand or right hand notation. Thus $q_{A / B}$ gives the orientation of the frame $B$ with respect to frame $A$. The quaternion is defined as shown in equation 16 . The quaternions do not suffer from the singularity problem and are easier to interpolate.

$$
\begin{gathered}
q_{A / B}=\left[\begin{array}{l}
q_{1} \\
q_{2} \\
q_{3} \\
q_{4}
\end{array}\right]=\left[\begin{array}{c}
r_{x} \sin (\theta / 2) \\
r_{y} \sin (\theta / 2) \\
r_{z} \sin (\theta / 2) \\
\cos (\theta / 2)
\end{array}\right] \\
r_{x}^{2}+r_{y}^{2}+r_{z}^{2}=1
\end{gathered}
$$

Quaternions are further constrained by equation 17, where $q^{T}$ is the quaternion transpose. Quaternion conjugate, denoted with ${ }^{\prime} *^{\prime}$ on the superscript, describes the orientation of the frame $A$ with respect to frame $B$ and is given by equation 18 .

$$
\begin{gathered}
q q^{T}=q_{1}^{2}+q_{2}^{2}+q_{3}^{2}+q_{4}^{2}=1 \\
q_{B / A}=q_{A / B}^{*}=\left[\begin{array}{c}
-q_{1} \\
-q_{2} \\
-q_{3} \\
q_{4}
\end{array}\right]
\end{gathered}
$$


The quaternion product is used to find the compound orientation. If two quaternions $q_{B / C}$ and $q_{A / B}$ are known, then the $q_{A / C}$ can be found using equation 19.

$$
q_{A / C}=q_{B / C} \otimes q_{A / B}
$$

The quaternion product is not commutative, i.e., $q_{B / C} \otimes q_{A / B} \neq q_{A / B} \otimes q_{B / C}$. In matrix form, equation 19 can be written as 20 (using the Hamilton theorem).

$$
\left[\begin{array}{l}
q_{1} \\
q_{2} \\
q_{3} \\
q_{4}
\end{array}\right]_{A / C}=\left[\begin{array}{cccc}
q_{4} & q_{3} & -q_{2} & -q_{1} \\
-q_{3} & q_{4} & q_{1} & -q_{2} \\
q_{2} & -q_{1} & q_{4} & -q_{3} \\
q_{1} & q_{2} & q_{3} & q_{4}
\end{array}\right]_{B / C}\left[\begin{array}{l}
q_{1} \\
q_{2} \\
q_{3} \\
q_{4}
\end{array}\right]_{A / B}
$$

If $q_{A / B}$ is known, then a three-dimensional vector in frame $A\left(\vec{v}^{A}\right)$ can be converted into frame $B$ using equation 21, while an additional zero is added as the fourth element of the vector in frame $A\left(\vec{v}^{A}\right)$ and in frame $B\left(\vec{v}^{B}\right)$. Equation 21 can be represented in the form of a rotation matrix as shown in equation 22 .

$$
\begin{gathered}
\vec{v}^{B}=q_{A / B} \otimes \vec{v}^{A} \otimes q_{A / B}^{*} \\
{\left[\begin{array}{l}
\vec{b}_{1} \\
\vec{b}_{2} \\
\vec{b}_{3}
\end{array}\right]=\left[\begin{array}{lll}
1-2\left(q_{2}^{2}+q_{3}^{2}\right) & 2\left(q_{1} q_{2}+q_{3} q_{4}\right) & 2\left(q_{1} q_{3}-q_{2} q_{4}\right) \\
2\left(q_{1} q_{2}-q_{3} q_{4}\right) & 1-2\left(q_{1}^{2}+q_{3}^{2}\right) & 2\left(q_{2} q_{3}+q_{1} q_{4}\right) \\
2\left(q_{1} q_{3}+q_{2} q_{4}\right) & 2\left(q_{2} q_{3}-q_{1} q_{4}\right) & 1-2\left(q_{1}^{2}+q_{2}^{2}\right)
\end{array}\right]\left[\begin{array}{l}
\vec{a}_{1} \\
\vec{a}_{2} \\
\vec{a}_{3}
\end{array}\right]} \\
C^{B / A}=\left[\begin{array}{lll}
1-2\left(q_{2}^{2}+q_{3}^{2}\right) & 2\left(q_{1} q_{2}+q_{3} q_{4}\right) & 2\left(q_{1} q_{3}-q_{2} q_{4}\right) \\
2\left(q_{1} q_{2}-q_{3} q_{4}\right) & 1-2\left(q_{1}^{2}+q_{3}^{2}\right) & 2\left(q_{2} q_{3}+q_{1} q_{4}\right) \\
2\left(q_{1} q_{3}+q_{2} q_{4}\right) & 2\left(q_{2} q_{3}-q_{1} q_{4}\right) & 1-2\left(q_{1}^{2}+q_{2}^{2}\right)
\end{array}\right]
\end{gathered}
$$

The rotation matrix shown in equation 22 can also be represented as equation 23 .

$$
\begin{gathered}
C^{B / A}=\left(q_{4}^{2}-\hat{q}^{T} \hat{q}\right) I+\hat{q} \hat{q}^{T}-2 q_{4} \hat{Q} \\
\text { Where }: \hat{q}=\left[\begin{array}{l}
q_{1} \\
q_{2} \\
q_{3}
\end{array}\right], \hat{Q}=\left[\begin{array}{ccc}
0 & -q_{3} & q_{2} \\
q_{3} & 0 & -q_{1} \\
-q_{2} & q_{1} & 0
\end{array}\right]
\end{gathered}
$$

The relationship between the Euler angles and the quaternion for the 2-3-1 Euler rotation is given by equation 24 .

$$
\begin{gathered}
{\left[\begin{array}{l}
q_{1} \\
q_{2} \\
q_{3} \\
q_{4}
\end{array}\right]=\left[\begin{array}{c}
c\left(\theta_{1} / 2\right) s\left(\theta_{2} / 2\right) s\left(\theta_{3} / 2\right)+s\left(\theta_{1} / 2\right) c\left(\theta_{2} / 2\right) c\left(\theta_{3} / 2\right) \\
c\left(\theta_{1} / 2\right) s\left(\theta_{2} / 2\right) c\left(\theta_{3} / 2\right)+s\left(\theta_{1} / 2\right) c\left(\theta_{2} / 2\right) s\left(\theta_{3} / 2\right) \\
c\left(\theta_{1} / 2\right) c\left(\theta_{2} / 2\right) s\left(\theta_{3} / 2\right)-s\left(\theta_{1} / 2\right) s\left(\theta_{2} / 2\right) c\left(\theta_{3} / 2\right) \\
c\left(\theta_{1} / 2\right) c\left(\theta_{2} / 2\right) c\left(\theta_{3} / 2\right)-s\left(\theta_{1} / 2\right) s\left(\theta_{2} / 2\right) s\left(\theta_{3} / 2\right)
\end{array}\right]} \\
\text { where, } c\left(\theta_{i} / 2\right)=\cos \left(\theta_{i} / 2\right) \\
s\left(\theta_{i} / 2\right)=\sin \left(\theta_{i} / 2\right) \\
q_{1}^{2}+q_{2}^{2}+q_{3}^{2}+q_{4}^{2}=1
\end{gathered}
$$


Furthermore, the inverse relationship is given by equation 26 .

$$
\begin{aligned}
& \theta_{1}=\tan ^{-1}\left\{\frac{2\left(q_{1} q_{4}-q_{2} q_{3}\right)}{1-2 q_{1}^{2} 2 q_{3}^{2}}\right\} \\
& \theta_{2}=\tan ^{-1}\left\{\frac{2\left(q_{2} q_{4}-q_{1} q_{3}\right)}{1-2 q_{2}^{2} 2 q_{3}^{2}}\right\} \\
& \theta_{3}=\sin ^{-1}\left\{2\left(q_{1} q_{2}-q_{3} q_{4}\right)\right\}
\end{aligned}
$$

\subsection{Discussion}

Among the three attitude representation schemes, the quaternion is highly preferred. Because they do not suffer from singularity problems, the quaternions are easy to interpolate, which leads to lower computational costs. Furthermore, the matrix representation and Euler angles leads to a large floating point error due to the rounding off of the sine and cosine functions. Restoring a rotation matrix is computationally more expensive than normalising the quaternion. Although quaternions are not intuitive and might feel mathematically complex, their advantages outweigh their disadvantages for practical applications such as the quadcopter, due to the resulting high maneuverability of the vehicle. Thus, the quadcopter model that will be used and the attitude determination filter that will be developed in this thesis will be using the quaternion representation scheme to represent the attitude of the quadcopter. 


\section{The mathematical model of a quadcopter}

\section{Introduction}

In this chapter, the dynamic and kinematic models of the quadcopter are discussed. The kinematic model is represented in terms of quaternion. It describes the mathematical model of the quadcopter without considering the cause of the motion, i.e., the applied forces on the system. The kinematic model provides us with the relationship between the angular velocity, measured in the body frame of reference, and the quaternion rate in the inertial reference frame. Two dynamic models of the quadcopter are discussed in the chapter: first the simple or reduced model that is derived by making the assumption that the aerodynamic forces acting on the quadcopter are negligible, i.e., the velocity of the quadcopter is very low. The reduced model is obtained from Benić et al. (2016). The second model is the more advance model, based on the research by Sun et al. (2018a). The quadcopter used in this research is the same quadcopter whose model has been developed by Sun et al. (2018a). Furthermore, the chapter discusses the accelerometer measurement model, assuming that it is not situated in the center of gravity of the quadcopter, and explains the variation in the reading of the accelerometer for this case.

\subsection{A quadcopter's kinematics model}

The kinematic model proves the relationship between the body frame transitional and angular velocities and the inertial frame transitional and angular velocities (for local flights, the inertial frame is the same as the navigation frame). $[\dot{x}, \dot{y}, \dot{z}]^{T}$ represent the linear velocity along the $x, y$ and $z$ axis of the inertial frame and $[\dot{\phi}, \dot{\theta}, \dot{\psi}]^{T}$ represent the angular velocity along the $x, y$ and $z$ axis of the inertial frame respectively. $[u, v, w]^{T}$ represent the linear velocity along the $x, y$ and $z$ axis of the body frame and $[p, q, r]^{T}$ represent the angular velocity along the $x, y$ and $z$ axis of the body frame respectively.

The kinematic equation for the linear velocity is given by equation 27 .

$$
\begin{gathered}
\vec{V}_{I}=C_{I / B} \vec{V}_{B} \\
\text { where }: C_{I / B}=\left[\begin{array}{ccc}
1-2\left(q_{2}^{2}+q_{3}^{2}\right) & 2\left(q_{1} q_{2}+q_{3} q_{4}\right) & 2\left(q_{1} q_{3}-q_{2} q_{4}\right) \\
2\left(q_{1} q_{2}-q_{3} q_{4}\right) & 1-2\left(q_{1}^{2}+q_{3}^{2}\right) & 2\left(q_{2} q_{3}+q_{1} q_{4}\right) \\
2\left(q_{1} q_{3}+q_{2} q_{4}\right) & 2\left(q_{2} q_{3}-q_{1} q_{4}\right) & 1-2\left(q_{1}^{2}+q_{2}^{2}\right)
\end{array}\right], \vec{V}_{I}=\left[\begin{array}{c}
\dot{x} \\
\dot{y} \\
\dot{z}
\end{array}\right], \vec{V}_{B}=\left[\begin{array}{c}
u \\
v \\
w
\end{array}\right]
\end{gathered}
$$

The kinematic equation for the angular velocity is given by equation 28 .

$$
\dot{q}_{I}=\frac{1}{2} q_{I} \otimes\left[\begin{array}{ll}
\vec{\omega}_{B} & 0
\end{array}\right]^{T}
$$

Equation 28 can also be represented as equation 29 in matrix form.

$$
\left[\begin{array}{l}
\dot{q}_{1} \\
\dot{q}_{1} \\
\dot{q}_{1} \\
\dot{q}_{1}
\end{array}\right]_{I}=\frac{1}{2}\left[\begin{array}{cccc}
q_{4} & -q_{3} & q_{2} & q_{1} \\
q_{3} & q_{4} & -q_{1} & q_{2} \\
-q_{2} & q_{1} & q_{4} & q_{3} \\
-q_{1} & -q_{2} & -q_{3} & q_{4}
\end{array}\right]_{I}\left[\begin{array}{c}
p \\
q \\
r \\
0
\end{array}\right]_{B}
$$

\subsection{A quadcopter's dynamic model}

Using Newton's law, equation 30 can be derived.

$$
m\left(\dot{\vec{V}}_{B}+\vec{\omega}_{B} \times \vec{V}_{B}\right)=\vec{F}_{B}
$$

where $\vec{F}_{B}$ is the net force vector in the body frame. Similarly, the movement equation for the quadcopter can be derived as equation 31 .

$$
I \cdot \dot{\vec{\omega}}_{B}+\vec{\omega}_{B} \times\left(I \cdot \vec{\omega}_{B}\right)=\vec{M}_{B}
$$


where $\vec{M}_{B}$ is the moment vector in the body frame of reference and $I$ is the inertia matrix of the quadcopter. Equation 30 and 31 can be expanded as equation 32.

$$
\begin{gathered}
f_{x_{B}}=m(\dot{u}+q w-r v) \\
f_{y_{B}}=m(\dot{v}-p w+r u) \\
f_{z_{B}}=m(\dot{w}+p v-q u) \\
L=m_{x_{B}}=\dot{p} I_{x}-I_{x z} \dot{r}+q r\left(I_{z}-I_{y}\right)-I_{x z} p q \\
M=m_{y_{B}}=\dot{q} I_{y}+r p\left(I_{x}-I_{z}\right)+I_{x y}\left(p^{2}-q^{2}\right) \\
N=m_{z_{B}}=-\dot{p} I_{x z}+I_{z} \dot{r}+p q\left(I_{y}-I_{x}\right)+I_{x z} q r
\end{gathered}
$$

This equation assumes that the origin of the body reference frame is located at the center of gravity of the quadcopter and that the quadcopter is symmetric about $x_{B} y_{B}$ plane, i.e., $I_{x y}=I_{y x}=0$.

\section{Forces and moments}

The vector $\vec{F}_{B}$ in equation 30 is the net external force acting on the quadcopter in the body frame of reference. This force is given as equation 33 .

$$
\vec{F}_{B}=m \vec{g}_{B}-\vec{F}_{T_{B}}+\vec{F}_{W_{B}}
$$

where $\vec{g}_{B}$ is the gravity vector in the body frame of reference, $\vec{F}_{T_{B}}=F_{T}\left[\begin{array}{lll}0 & 0 & 1\end{array}\right] \hat{E}_{B}, \hat{E}_{B}$ is the unit vector in the body frame of reference and $\vec{F}_{W_{B}}$ is the aerodynamics force in the body frame of reference.

If the rotation matrix in known $\left(R_{B / I}\right)$, the force due to gravity can be represented as 34 , where $\left[\hat{E}_{I}\right]$ is the unit vector in the inertial frame.

$$
\begin{gathered}
\vec{F}_{g B}=m \vec{g}_{B}=m|g|\left[\begin{array}{lll}
0 & 0 & 1
\end{array}\right] R_{B / I}\left[\hat{E}_{I}\right] \\
{\left[\hat{E}_{I}\right]=\left[\begin{array}{lll}
\hat{x}_{i} & \hat{y}_{i} & \hat{z}_{i}
\end{array}\right]^{T}}
\end{gathered}
$$

The thrust and aerodynamic forces are modeled in two ways, namely simple and complex. A simple model is based on the assumption that quadcopters have a very small velocity and thus all the aerodynamic forces are neglected (assumed to be zero). Equation 35 gives the simple model.

$$
\begin{gathered}
\vec{F}_{T_{B}}=K\left[\begin{array}{ccc}
0 & 0 & \sum_{i=1}^{4} \omega_{i_{\text {rot }}}^{2}
\end{array}\right]^{T}\left[\hat{E}_{B}\right] \\
\vec{F}_{W_{B}} \approx 0
\end{gathered}
$$

where $\hat{E}_{B}$ is the unit vector in the body reference frame, $\omega_{\text {irot }}$ is the angular velocity of the $i_{t h}$ rotor and $\mathrm{K}$ is a constant whose value depends on the design of the rotor. The more accurate force model obtained in the paper by Sun et al. (2018a) is given in equation 36 .

$$
\begin{gathered}
F_{B_{Z}}=K_{0} \sum \omega_{i}^{2}+F_{W_{Z}} \\
F_{W_{Z}}=Z_{0}+Z_{1} w \sum \omega_{i}+Z_{2} u^{2}+Z_{3} v^{2}+Z_{4} u^{2} w+Z_{5} v w^{2}+Z_{6} u^{2} \sum \omega_{i} \\
F_{B_{X}}=X_{0}+X_{1} u \sum \omega_{i}+X_{2}|v| w+X_{3}|v| u+X_{4} u w^{2}+X_{5} u w \sum \omega_{i}+X_{6} u w^{2}+X_{7}|v|^{2} u
\end{gathered}
$$

The values of the parameters are given in table 4.1 and 4.2 . 


\begin{tabular}{l|l|l|}
\multicolumn{1}{l}{ Parameter } & \multicolumn{1}{c}{ Value } & \multicolumn{1}{c|}{$\sigma$} \\
\hline$K_{0}$ & $1.58 e^{-6}$ & \\
\hline$Z_{0}$ & $-2.55 e^{-1}$ & $5.79 e^{-6}$ \\
\hline$Z_{1}$ & $9.41 e^{-5}$ & $1.05 e^{-6}$ \\
\hline$Z_{2}$ & $7.39 e^{-3}$ & $8.40 e^{-6}$ \\
\hline$Z_{3}$ & $6.56 e^{-3}$ & $1.20 e^{-6}$ \\
\hline$Z_{4}$ & $2.74 e^{-3}$ & $6.37 e^{-6}$ \\
\hline$Z_{5}$ & $-1.58 e^{-3}$ & $6.37 e^{-6}$ \\
\hline$Z_{6}$ & $2.76 e^{-6}$ & $3.02 e^{-6}$ \\
\hline
\end{tabular}

Table 4.1: Estimated values for the parameters (Sun et al. (2018a))

\begin{tabular}{|l|l|l|}
\multicolumn{1}{l}{ Parameter } & \multicolumn{1}{c}{ Value } & \multicolumn{1}{c}{$\sigma$} \\
\hline$X_{0}$ & $7.47 e^{-2}$ & $9.82 e^{-5}$ \\
\hline$X_{1}$ & $-3.59 e^{-5}$ & $1.26 e^{-8}$ \\
\hline$X_{2}$ & $-6.88 e^{-4}$ & $4.17 e^{-6}$ \\
\hline$X_{3}$ & $-2.00 e^{-3}$ & $1.87 e^{-5}$ \\
\hline$X_{4}$ & $7.79 e^{-9}$ & $3.10 e^{-9}$ \\
\hline$X_{5}$ & $1.83 e^{-6}$ & $8.18 e^{-9}$ \\
\hline$X_{6}$ & $1.15 e^{-3}$ & $1.34 e^{-5}$ \\
\hline$X_{7}$ & $1.62 e^{-4}$ & $2.29 e^{-6}$ \\
\hline$X_{8}$ & $3.28 e^{-5}$ & $6.66 e^{-7}$ \\
\hline
\end{tabular}

Table 4.2: Estimated values for the parameters (Sun et al. (2018a))

Similarly, external moments in the body frame are given by equation 37 , where $\vec{M}_{\text {motor }_{B}}$ is the control moment due to the speed of the rotors, $\vec{M}_{g_{y r o}}$ is the gyroscopic moment due to the rotating rotor and the quadcopter and $\overrightarrow{M_{W_{B}}}$ is the moment due to the wind. These moments are also given by two models, namely the simple and the complex model.

$$
\vec{M}_{B}=\vec{M}_{\text {motor }_{B}}+\vec{M}_{\text {gyro }_{B}}+\vec{M}_{W_{B}}
$$

In the simple model, the moment due to the gyroscopic principle and wind are ignored, as the weight of the rotor is very low and the wind speed is considered small. The simple model's external moment is given by equation 38 .

$$
\begin{gathered}
\vec{M}_{B}=\left[\begin{array}{l}
m_{B_{x}} \\
m_{B_{y}} \\
m_{B_{z}}
\end{array}\right]=\left[\begin{array}{c}
b l\left(\omega_{\text {rot }_{3}}^{2}-\omega_{\text {rot }_{1}}^{2}\right) \\
b l\left(\omega_{\text {rot }_{4}}^{2}-\omega_{\text {rot }_{2}}^{2}\right) \\
d\left(\omega_{\text {rot }_{2}}^{2}+\omega_{\text {rot }_{4}}^{2}-\omega_{\text {rot }_{3}}^{2}-\omega_{\text {rot }_{1}}^{2}\right)
\end{array}\right] \\
b=C_{T} \rho A r^{2} \\
d=C_{P} \rho A r^{3}
\end{gathered}
$$

where $b$ is thrust coefficient measured in $N s^{2}, l$ is the distance between the center of gravity and the propeller axis, $C_{T}$ is the thrust factor, $C_{P}$ is the power factor, $\rho$ is the air density, $A$ is the area of propeller disc, $r$ is the radius of the propeller and $d$ is the drag coefficient. The complex model moments equation is given by equation 39 .

$$
\begin{gathered}
M_{B_{y}}=b K_{0} U_{q}+M_{0}+M_{1} u_{2} U_{q} M_{3} w U_{q}+M_{4} u+M_{5} u_{3}+M_{6} w+M_{7} u w U_{q}+ \\
M_{8} q+M_{9} u \sum \omega_{i}+M_{10} u^{2} \sum \omega_{i}+M_{11} u w \\
M_{B_{z}}=M_{h}+N_{0}+N_{1} v+N_{2} u U_{r}+N_{3} u^{3} U_{r}+N_{4} v^{2} U_{r}+N_{5} u v
\end{gathered}
$$


where:

$$
\begin{gathered}
M_{h}=\tau_{0} U_{r}+I_{r, z}\left(-\dot{\omega}_{1}+\dot{\omega}_{2}-\dot{\omega}_{3}+\dot{\omega}_{4}\right)+\gamma r \\
U_{q}=\omega_{1}^{2}+\omega_{2}^{2}-\omega_{3}^{2}-\omega_{4}^{2} \\
U_{r}=\omega_{1}^{2}-\omega_{2}^{2}+\omega_{3}^{2}-\omega_{4}^{2}
\end{gathered}
$$

The values of the parameters are given in tables 4.3 and 4.4.

\begin{tabular}{|l|l|l|}
\multicolumn{1}{l}{ Parameter } & \multicolumn{1}{c}{ Value } & \multicolumn{1}{c}{$\sigma$} \\
\hline$M_{0}$ & $1.05 e^{-3}$ & $6.47 e^{-5}$ \\
\hline$M_{1}$ & $-6.53 e^{-9}$ & $8.73 e^{-11}$ \\
\hline$M_{2}$ & $-5.72 e^{-10}$ & $1.03 e^{-11}$ \\
\hline$M_{3}$ & $-3.05 e^{-8}$ & $1.92 e^{-10}$ \\
\hline$M_{4}$ & $-8.42 e^{-3}$ & $8.93 e^{-5}$ \\
\hline$M_{5}$ & $3.59 e^{-5}$ & $6.41 e^{-7}$ \\
\hline$M_{6}$ & $8.67 e^{-3}$ & $1.36 e^{-4}$ \\
\hline$M_{7}$ & $3.08 e^{-9}$ & $2.41 e^{-11}$ \\
\hline$M_{8}$ & $-3.74 e^{-3}$ & $2.40 e^{-5}$ \\
\hline$M_{9}$ & $8.78 e^{-6}$ & $4.29 e^{-8}$ \\
\hline$M_{10}$ & $-4.67 e^{-7}$ & $4.21 e^{-9}$ \\
\hline$M_{11}$ & $6.69 e^{-4}$ & $1.82 e^{-5}$ \\
\hline
\end{tabular}

Table 4.3: Estimated values for the parameters (Sun et al. (2018a))

\begin{tabular}{|l|l|l|}
\multicolumn{1}{l}{ Parameter } & \multicolumn{1}{c}{ Value } & \multicolumn{1}{c}{$\sigma$} \\
\hline$\tau_{0}$ & $1.89 e^{-8}$ & $3.83^{-11}$ \\
\hline$I_{r, z}$ & $3.34^{-6}$ & $5.51^{-9}$ \\
\hline$\gamma$ & $-9.57^{-4}$ & $1.45^{-5}$ \\
\hline$N_{0}$ & $5.67^{-4}$ & $1.02^{-5}$ \\
\hline$N_{1}$ & $-2.18^{-4}$ & $5.59^{-6}$ \\
\hline$N_{2}$ & $-1.71^{-9}$ & $1.45^{-11}$ \\
\hline$N_{3}$ & $1.52^{-11}$ & $1.69^{-13}$ \\
\hline$N_{4}$ & $-8.53^{-11}$ & $5.52^{-13}$ \\
\hline$N_{5}$ & $-1.10^{-4}$ & $1.30^{-6}$ \\
\hline
\end{tabular}

Table 4.4: Estimated values for the parameters (Sun et al. (2018a))

\subsection{Measurement Model}

Measurements made by the accelerometer are equal to the specific force applied on the point where the accelerometer is situated. Thus, if the accelerometer is placed in the center of mass and the center of rotation, then the specific force is equal to equation 40 .

$$
\vec{a}_{s p_{B}}=R_{B / I}\left(\vec{a}_{I}-\vec{g}_{I}\right)
$$

where $\vec{a}_{s p_{B}}$ is the specific acceleration in the body frame of reference, $\vec{a}_{I}$ is the acceleration of the center of mass of the quadcopter in the inertial frame and $\vec{g}_{I}$ is the gravity vector in the inertial frame of reference. On the other hand, if the accelerometer is not situated at the center of mass, then the specific acceleration measured is given by equation 41 .

$$
\vec{a}_{s p_{B}}=\vec{a}_{B}+\dot{\vec{\omega}}_{B} \times \vec{r}_{B}+2 \vec{\omega}_{B} \times \dot{\vec{r}}_{B}+\vec{\omega}_{B} \times\left(\vec{\omega}_{B} \times \vec{r}_{B}\right)+\vec{g}_{B}
$$


where $\vec{r}_{B}$ is the distance of the accelerometer from the center of mass in the body frame of reference, $\vec{\omega}_{B}$ is the body frame angular velocity with respect to the inertial frame expressed in the body reference frame, $g_{B}$ is the acceleration due to gravity in the body frame of reference and $\vec{a}_{B}$ is the acceleration of the center of mass in the body frame of reference.

Thus, under the assumption that the position of the accelerometer is constant and the quadcopter's angular acceleration is small or very short-lived, the equation 41 reduces to equation 42 .

$$
\vec{a}_{s p_{B}} \approx \vec{a}_{B}+\vec{\omega}_{B} \times\left(\vec{\omega}_{B} \times \vec{r}_{B}\right)+\vec{g}_{B}
$$

Using this measurement model, the accelerometer measurements given in equation 9 can be updated to equation 43 .

$$
y_{\text {accel }_{B}}=R_{B I}\left(a_{I}+g_{I}\right)+\vec{\omega}_{B} \times\left(\vec{\omega}_{B} \times \vec{r}_{B}\right)+e_{\text {accel }}+\delta_{\text {accel }, t}
$$

where $e_{a}$ is the random noise of the sensor and $\delta_{a, t}$ is the time-varying bias of the accelerometer. It is assumed that the random noise of the accelerometer $\left(e_{a}\right)$ is Gaussian white noise with zero mean and having a standard deviation $\Sigma_{a}$. As can be seen from the definition of the $\Sigma_{a}$, it is assumed that there is no correlation between the three axes.

$$
\Sigma_{a}=\left(\begin{array}{ccc}
\sigma_{a, x} & 0 & 0 \\
0 & \sigma_{a, y} & 0 \\
0 & 0 & \sigma_{a, z}
\end{array}\right)
$$

Furthermore, using the same logic, equation 30 can be modified for the position where the accelerometer is situated as equation 44 .

$$
m\left(\dot{\vec{V}}_{B}+\vec{\omega}_{B} \times \vec{V}_{B}\right)+\vec{\omega}_{B} \times\left(\vec{\omega}_{B} \times \vec{r}_{B}\right)=\vec{F}_{B}
$$




\section{A classification of state estimation methods}

\section{Introduction}

In this chapter, a broad classification of the state estimation methods is provided. The classification is loosely based on survey papers by Jin et al. (2019), Crassidis et al. (2007b) and Guo et al. (2018). The classification is done in such a way that it provides a broad overview of all the different types of vehicle state estimation methodologies. Figure 5.1 shows the classification in a chart format.

Vehicle state estimation methodologies can be divided into two main categories, namely vehicle model-based estimation and data-driven-based estimation. The vehicle model-based estimation can further be divided into two main subcategories, namely kinematic model-based estimation methods and dynamic model-based estimation methods. The dynamic model-based estimation methods can further be divided into filter-based estimation methods and observer-based estimation methods. Filter-based estimation is further classified in multiple categories such as deterministic approach, complimentary filter based approach and stochastic approach. These three are explored in detail in chapters 5, 6 and 7 respectively. While a brief overview of on data-driven based methods and observer based methods is provided in this chapter along with a discussion of their respective advantages and disadvantage, drawing from the literature, as well as the reasons for selecting a particular branch of methods for this thesis.

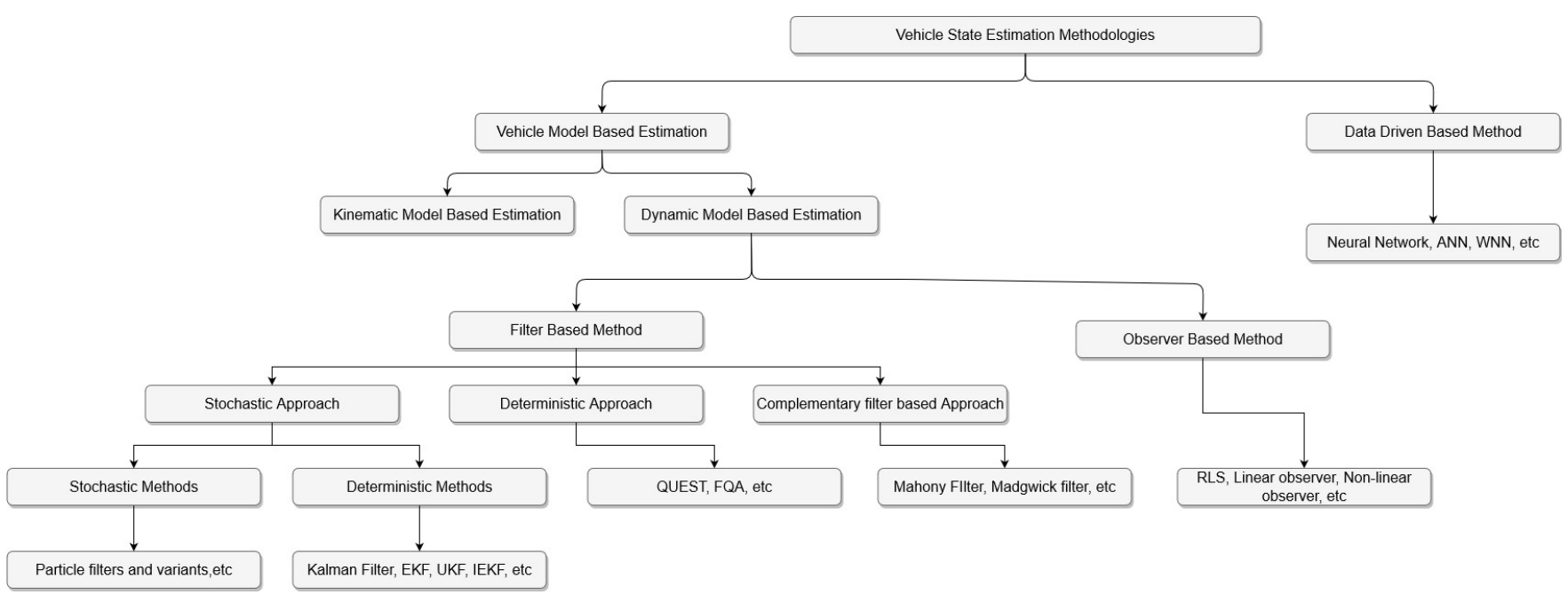

Figure 5.1: Classification of vehicle attitude estimation methodologies

\subsection{Data driven-based estimation}

The data-driven-based estimation approach uses the already available input/output data to develop an estimator that is able to estimate the states of the system. These techniques obtain the knowledge about a system's dynamic by processing the already available input/output data sheets. Thus, these technique do not depend on the reference system, but are mostly black box or gray box estimators. Among the available data driven approaches, the ANN (Artificial neural network)-based approach is the most famous for estimating the vehicle states. These techniques are a mixture of the new intelligent approaches and conventional estimation methods. ANN is a technique that is inspired by animals' nervous systems. A wide number of these techniques are used in state estimation of ground vehicles such as cars. 


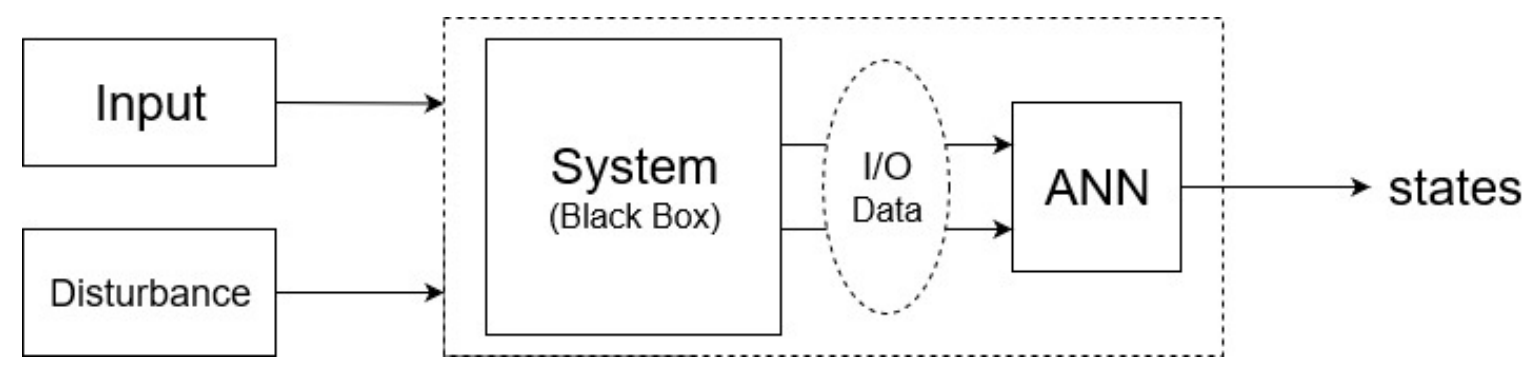

Figure 5.2: The schematic of ANN estimation process

The data-driven state estimation methods have shown promising results in various fields, such ground vehicles (Dong et al. (2015)), UAVs (Hatamleh et al. (2015)), and under-water vehicles (Chang et al. (2017)). In (Saadeddin et al. (2013b)), an adaptive neuro-fuzzy inference system with an input delay technique $(A N F I S)$ was designed to estimate the system's velocity and position. This was achieved via data-set fission of the GPS and inertial navigation system. Neural networks are also used for multiple sensor fusion, in (Nguyen-H and Zhou (2010)) INS and GPS integration was performed using neural networks, while neural-network-based MMF was developed by (Gwak et al. (2013)) to obtain the accurate position estimate using the on-board sensors and GPS. Multiple ANN techniques have been developed to estimate the vehicle side-slip angle. In (Boada et al. (2015b), Boada et al. (2016a)), (ANFIS) was used to estimate a pseudo-side slip angle from the measurements of the steering wheel and inertial sensors.

Multiple data-driven-based estimation techniques have been developed in recent years, such as (ANFIS) (Artificial neural fuzzy inference system) estimator (Saadeddin et al. (2013a), Boada et al. (2015a), Boada et al. (2016b)), GRNN (general regression neural network) estimator (Wei et al. (2016)), deep-learning based estimator (Kim and Bae (2019)), and many more. Although these estimators are very promising, they require a lot of processing power and in many cases the estimator needs to be trained beforehand by using preexisting data. Furthermore, it is very hard to predict the reliability of the estimators, especially for untrained cases.

The aim of this thesis is to develop an estimator to estimate the states of a quadcopter in failure mode. Mostly quadcopters do not posses a high processing power. Furthermore, there is not much data available for the training of the estimator. Thus, a data-driven-based estimator is not the best estimator for this case.

\subsection{Vehicle model-based estimation}

As mentioned in the previous section, vehicle model-based estimation is further classified into kinematic model-based estimation methods and dynamic model-based estimation methods. Kinematic model-based estimation uses kinematic relationships between the estimated states and the measured sensor data to estimate the dynamic states. Some studies relating to this concept can be found. For example, in Hac and Simpson (2000) the relationship between the yaw rate, lateral acceleration and lateral velocity derivative is used to obtain the lateral velocity of the vehicle via integration. Kinematic model-based estimation uses mainly the integration of the sensor measurements to obtain the states of the system. Since sensor readings are always corrupted with noise, time-varying bias and other errors and since the kinematic model-based estimation is based on sensor reading integration, the estimate obtained is usually very distorted and far from the the real estimate. Thus, the majority of the studies tend to study the dynamic model-based estimation methods, as they use the system model along with other sensor readings to obtain the system state estimate.

\subsection{Dynamic-model based method}

Dynamic model-based estimation methods are divided into two categories, namely filter-based and observer-based methods. These techniques use the available dynamic system model information 
in one way or another to estimate the states of the system. The dynamic model of the system describes the physical and mathematical model characteristics of the system.

\subsection{Observer-based methods}

If a system is deterministic in nature, then the system's dynamic state estimation can be expressed as an observer design problem. A correction term is introduced to cater for the various errors (modelling error, sensor and system noise, etc.), which is based on the relationship between the states and the output of the system. One type of observer-based method is the Luenberger observer. In order to estimate the system's roll and pitch angle, an IMU and two kinematics-based Luenberger observers are used (Eric Tseng et al. (2007)).

This observer is suited for linear systems, but when the system turns nonlinear, the estimated states do not converge to the true values. Further research has been done on nonlinear observerbased methods. They are based on the Luenberger observer. Imsland et al. (2006) use a nonlinear observer in order to estimate a vehicle's velocity and yaw rate. Another similar nonlinear filter is introduced by Zhao et al. (2011). There is no completely clear boundary between the complementary filter based methods and the observer-based methods, which means that many authors consider filters such as the Mahony filter to be an observer-based method. Some of the observerbased methods were used in state estimation of ground vehicles and are not directly applicable to quadcopters. They will be briefly summed up in the following paragraphs. The few methods that are sometimes classified as observer-based and are relevant for aerial vehicles will be classified as complimentary filter based methods for the purpose of this thesis, and will be discussed in the respective chapter.

Observers based on the Luenberger observer use a method with a non-linear observer to estimate a car's velocity and yaw rate, thus providing the usually unstable ISS estimation results. Rajamani et al. (2011) use an observer that is based on a roll dynamic model in order to estimate the roll angle. Furthermore, Grip et al. (2009) and Gao et al. (2010) consider the varying road friction coefficient as discuss an adaptive NLO as a solution to the sideslip angle problem. Imsland et al. (2007) introduces a similar solution, providing an NLO to estimate a car's velocity that also considers the road bank angle. The advantage of the NLO is on the one hand its high flexibility and the fact that it produces various observations based on different models of cars, and on the other hand a much lower computational cost than the EKF while providing a performance of similar quality. When applied to an actual car, it provides a satisfactory real-time performance, unlike the optimal estimation method, and is furthermore much simpler in structure than the EKF.

The drawbacks of the NLO are its high fragility when parameter condition vary, which however can be mitigated by using an unknown input observer (UIO), such as described by Imsland et al. (2007) for the case of lateral velocity estimation on banked roads. The authors of that paper draw the conclusion that the structure of error dynamics in the nonlinear UIO are similar to a nonlinear observer which does not have known inputs.

Another frequently used method for the estimation of vehicle dynamic states is SMO, which has the advantage of a low number of calculations and an easy design procedure. It is also able to force a system into a sliding mode surface and thus reconstruct the state. Stephant et al. (2004) use it for the estimation of a sideslip angle and argues in favour of combining a validated simulator and experimental data gathered under lab-conditions. Furthermore, Oudghiri et al. (2007) estimate the lateral velocity with a structure that introduces a sliding mode term, which proves to be robust against sensor noise, and, for a change in the condition of road adhesion, subsequently representing the nonlinear vehicle model with an uncertain T-S fuzzy model.

Linear Matrix Inequalities (LMI) are used in these types of observers in order to express their stability conditions. Another advantage of SMO is that, through varying the structure control, it suppresses the model error's parameter uncertainty. The issue is to choose a suitable filter time because one needs to find a balance between minimizing the filtering time and making it long enough 
to be able to suppress the system's high-frequency noise. Finally, a high amount of repeated regulations are necessary for choosing the observer in order to meet the car's safety system's accuracy requirements, which is similar to the nonlinear observer. 


\section{Deterministic Approach}

\section{Introduction}

The deterministic approach is a purely mathematical approach to obtain the attitude of a system. The attitude of the system is considered as a quaternion or a matrix that transforms a vector in one frame into a vector in another frame. This attitude estimation algorithm uses the vector observed in the body reference frame to numerically obtain the matrix or quaternion that converts the observed vector into a known vector (vector in the inertial frame). Thus, the obtained matrix or quaternion gives the attitude of the system. This approach eliminates the need to consider the system's kinematics and dynamics and the attitude is determined using an optimising technique.

These algorithms are also called deterministic solutions. They are mainly single point algorithms, i.e., they determine the attitude of the system at the point of time when the observation of a known vector (inertial frame) is available in the body reference frame without considering any past information or observation. Although the computational cost of such a technique is low, it comes with a decreased accuracy, since past observations or system dynamics are not considered in determining the attitude. However, the more recently developed algorithms that are also recursive in nature consider the body dynamics in the attitude determination process and are very similar to Kalman filters.

\section{Wahba's problem}

Grace Wahba (Wahba (1966)) formulated the problem of obtaining a rotation matrix (SO3) between two reference frames, given the observation of weighted vectors in both the frames. The deterministic approach methods are solutions to Wahba's problem.

It is assumed that at least two sets of weighted observation vectors are available simultaneously in both frames, i.e., $\vec{a}_{I_{1}}, \vec{a}_{I_{2}}, \ldots \vec{a}_{I_{n}}$ and $\vec{a}_{B_{1}}, \vec{a}_{B_{2}}, \ldots \vec{a}_{B_{n}}$ with $n \geq 2$. Where $\vec{a}_{I_{i}}$ is the $i_{t h}$ vector in reference frame $I$ (inertial frame), and $\vec{a}_{B_{i}}$ is the $i_{\text {th }}$ vector in reference frame $B$ (body frame). The solution to Wahba's problem is also called the "batch attitude determination problem". The rotation matrix $(R)$ can be obtained by minimising the cost function given in equation 45 .

$$
L=\frac{1}{2} \sum_{i=1}^{n} w_{i}\left|\vec{a}_{B_{i}}-R \vec{a}_{I_{i}}\right|^{2}
$$

where $L$ is the cost function, $w_{i}$ is the non-negative weight and $n$ is the total number of simultaneously observed vectors. If normalised weights are used, i.e., $\sum_{i=1}^{n} w_{i}=1$, then equation 45 can be written as 46 .

$$
L=1-\sum_{i=1}^{n} w_{i}\left(\vec{a}_{B_{i}}\right)^{T} R \vec{a}_{I_{i}}=1-\operatorname{tr}\left(R Q^{T}\right)
$$

where $\operatorname{tr}$ is the trace operator and the $Q$ matrix is defined by equation 47 .

$$
Q=\sum_{i=1}^{n} w_{i} \vec{a}_{B_{i}}\left(\vec{a}_{I_{i}}\right)^{T}
$$

Thus, the problem is reduced to a maximization problem, where the term $\operatorname{tr}\left(Q^{T}\right)$ is maximised by finding the rotation matrix $R$. Wahba's problem discusses the attitude matrix determination in closed form, while a generalised problem is addressed by Psiaki (2010) and Shuster (2006).

Multiple methods such as in Farrell et al. (1966) and Keat (1977) have been developed to solve Wahba's problem. The most popular and most frequently used methods are discussed in the following sections of the chapter. 


\subsection{TRIAD}

TRIAD, which was first proposed by Lerner (1978) is one of the earlier methods to obtain a solution for Wahba's problem. The method requires two non-co-linear vectors in frame one and their respective observation vector in frame two. In the context of attitude determination, frame one is the inertial frame and the two non-co-linear vectors are called reference vectors, while frame two is the body reference frame. The reference vector and the observation vector are represented by $\vec{a}_{I_{1}}, \vec{a}_{I_{2}}$ and $\vec{a}_{B_{1}}, \vec{a}_{B_{2}}$ respectively. The vectors are used to obtain two ortho-normal frames with the bases $\hat{f}_{1}, \hat{f}_{2}, \hat{f}_{3}$ and $\hat{g}_{1}, \hat{g}_{2}, \hat{g}_{3}$, as shown in equation 48 .

$$
\begin{gathered}
\hat{f}_{1}=\vec{a}_{I_{1}} \\
\hat{f}_{2}=\frac{\vec{a}_{I_{1}} \times \vec{a}_{I_{2}}}{\left|\vec{a}_{I_{1}} \times \vec{a}_{I_{2}}\right|} \\
\hat{f}_{3}=\frac{\vec{a}_{I_{1}} \times\left(\vec{a}_{I_{1}} \times \vec{a}_{I_{2}}\right)}{\left|\vec{a}_{I_{1}} \times \vec{a}_{I_{2}}\right|} \\
\hat{g}_{1}=\vec{a}_{B_{1}} \\
\hat{g}_{2}=\frac{\vec{a}_{B_{1}} \times \vec{a}_{B_{2}}}{\left|\vec{a}_{B_{1}} \times \vec{a}_{B_{2}}\right|} \\
\hat{g}_{3}=\frac{\vec{a}_{B_{1}} \times\left(\vec{a}_{B_{1}} \times \vec{a}_{B_{2}}\right)}{\left|\vec{a}_{B_{1}} \times \vec{a}_{B_{2}}\right|}
\end{gathered}
$$

Then the rotation matrix is given by equation 49 .

$$
R=\sum_{i=1}^{3} \hat{f}_{i} \hat{g}_{i}^{T}
$$

The method is quite simple in nature, but a part of the observation is discarded, and thus optimal attitude determination methods usually perform better. The TRIAD algorithm furthermore works only with two vectors.

\subsection{SVD}

The Singular Value Decomposition (SVD) method was proposed as a solution to Wahba's problem by Markley (1988). Similar to the previously explained deterministic approach, SVD is also a point-to-point algorithm. Consequently, the algorithm only utilises the current observed vector to determine the attitude of the system while disregarding the system's model information. The $Q$ matrix in equation 47 is subjected to SVD as shown in equation 50.

$$
Q=U S V^{t}
$$

where $V$ and $U$ are orthogonal matrices and matrix $S$ is a diagonal matrix given by equation 51 .

$$
S=\left[\begin{array}{ccc}
s_{1} & 0 & 0 \\
0 & s_{2} & 0 \\
0 & 0 & s_{3}
\end{array}\right]
$$

The terms $s_{1}, s_{2}, s_{3}$ are the singular values of matrix $Q$ and follow equation 52 .

$$
s_{1} \geq_{2} \geq_{3}
$$

The author defines the proper orthogonal matrix and the diagonal matrix as given by equation 53 .

$$
U_{+}=U[\operatorname{diag}(1,1, \operatorname{det}(U))]
$$




$$
\begin{gathered}
V_{+}=V[\operatorname{diag}(1,1, \operatorname{det}(V))] \\
S^{\prime}=\operatorname{diag}\left(s_{1}, s_{2}, s_{3} \operatorname{det}(U) \operatorname{det}(V)\right)
\end{gathered}
$$

where the operator det is the determinant of the respective matrix. Furthermore, $\operatorname{det}(U), \operatorname{det}(V)=$ \pm 1 . Using this matrix, $Q$ can be decomposed as equation 54 and the optimal rotation matrix is given by equation 55 .

$$
\begin{gathered}
Q=U_{+} S^{\prime} V_{+}^{T} \\
R=U_{+} V_{+}=U[\operatorname{diag}(1,1, \operatorname{det}(U) \operatorname{det}(V))] V^{T}
\end{gathered}
$$

A similar algorithm called as FOAM (fast optimal attitude matrix) developed by Markley (1993) utilises the $\mathrm{Q}$ matrix properties to obtain the rotation matrix as given in equation 56 .

$$
R=\frac{\left(\kappa+\|Q\|^{2}\right) Q+\lambda(\operatorname{adj}(Q))^{T}-Q Q^{T} Q}{\xi}
$$

Where the operator adj denotes the ad-joint of a matrix, $\|Q\|^{2}, \kappa, \lambda$ and $\xi$ are given by equation 57 ( $\kappa, \lambda$ and $\xi$ are scalar).

$$
\begin{gathered}
\|Q\|^{2}=s_{1}^{2}+s_{2}^{2}+s_{3}^{2} \\
\kappa=s_{2} s_{3}+s_{1} s_{2}+s_{3} s_{2} \\
\lambda=s_{1}+s_{2}+s_{3} \\
\xi=\left(s_{2}+s_{3}\right)\left(s_{3}+s_{1}\right)\left(s_{1}+s_{2}\right)
\end{gathered}
$$

The FOAM algorithm, which is based entirely on SVD, is an iterative algorithm. Instead of calculating the diagonal elements of the $S$ matrix $\left(s_{1}, s_{2}, s_{3}\right)(51)$, the FOAM algorithm calculates the three scalars $(\kappa, \lambda$ and $\xi$ ) in an iterative manner. The coefficients $\kappa$ and $\xi$ can be expressed as equation 58.

$$
\begin{gathered}
\kappa=\frac{1}{2}\left(\lambda^{2}\|Q\|^{2}\right) \\
\xi=\kappa \lambda-\operatorname{det}(Q)
\end{gathered}
$$

Furthermore, $\lambda$ can be expressed as equation 59 .

$$
\lambda=\operatorname{tr}\left(R Q^{T}\right)
$$

Using equation 59 and equation $56, \lambda$ can be obtained via solving equation 60 recursively.

$$
\left(\lambda-\|Q\|^{2}\right)^{2}-8 \lambda \operatorname{det}(Q)-4\|\operatorname{adj} B\|^{2}=0
$$

After $\lambda$ is found, the other two scalars are computed using equation 58.

Compared to the previously explained methods, FOAM is the fastest and the most robust. However, just like the others, FOAM also has a problem when the system rotates $180^{\circ}$ (Markley and Mortari (2000), Mortari (1997)). 


\subsection{QUEST}

The rotation matrix can be written in the form of a quaternion, as shown in equation 61 .

$$
R=\left(q_{4}^{2}-q_{T} q\right) I_{3 \times 3}+2 q q^{T}-2 q_{4}[q \times]
$$

where

$$
\begin{gathered}
\bar{q}=\left[\begin{array}{ll}
q^{T} & q_{4}
\end{array}\right]^{T}, q=\left[\begin{array}{l}
q_{1} \\
q_{2} \\
q_{3}
\end{array}\right] \\
{[q \times]=\left[\begin{array}{ccc}
0 & -q_{3} & q_{2} \\
q_{3} & 0 & -q_{1} \\
-q_{2} & q_{1} & 0
\end{array}\right]}
\end{gathered}
$$

Equation 46 can be written as equation 62 .

$$
L=1-g(R)
$$

where $g(R)$ is the gain function. It is observable that $g(R)$ needs to be maximised to minimise $L$ (equation 46).

$$
g(R)=\sum_{i=1}^{n} w_{i}\left(\vec{a}_{B_{i}}\right)^{T} R \vec{a}_{I_{i}}
$$

Using equations 61 and 62 , the gain function can be written in quaternion representation as equation 63.

$$
g(\bar{q})=\bar{q}^{T} K \bar{q}
$$

where

$$
\begin{gathered}
K=\left[\begin{array}{cc}
S-\sigma I & Z \\
Z^{T} & \sigma
\end{array}\right] \\
Q=\sum w_{i}\left(a_{B_{i}} a_{I_{i}}^{T}\right) \\
S=Q+Q^{T} \\
Z=\sum w_{i}\left(a_{B_{i}} \times a_{I_{i}}\right) \\
\operatorname{tr}(Q)
\end{gathered}
$$

Furthermore, in quaternion representation $\bar{q}^{T} \bar{q}=1$. To maximise equation 63 one can show, using the Lagrangian multiplier method (subsequently differentiated with respect to $\bar{q}$ ), that the eigenvector of the largest positive eigenvalue of matrix $K$ is the one that is associated with the quaternion that maximises equation 63 .

$$
K \bar{q}_{o p}=\lambda_{\max } \bar{q}_{o p}
$$

where $\bar{q}_{o p}$ is the optimal quaternion that maximises equation 63 . Thus, the attitude of the system can be estimated by solving for the largest eigenvalue of matrix $K$. This converts the minimisation problem into an eigenvalue problem.

Multiple algorithms have been developed that aim to find the largest eigenvector of matrix $K$ and the associated eigenvalue. The algorithms such as "Estimation of Optimal quaternion" (ESOQ) 
(Markley and Mortari (2000), Mortari (1997)) and the most famous QUEST algorithm (Quaternion estimation) developed by Shuster and Oh (1981) fall into this category. QUEST is used for many real-time applications as in the QUEST algorithm there is no need to minimise the cost function to obtain the optimal quaternion $\left(\bar{q}_{o p}\right)$. There are multiple ways to obtain the eigenvector of a given matrix, but they are usually computationally expensive, and the QUEST algorithm provides a cheaper alternative to the eigenvalue problem. In the $Q U E S T$ algorithm, the CaylayHamilton theorem is applied on the $S$ Matrix (given in equation 63) to obtain equation 65.

$$
S^{3}=\operatorname{tr}(S) S^{2}-\operatorname{tr}(\operatorname{adj} S) S+\operatorname{det}(S) I_{3 \times 3}
$$

where $I_{3 \times 3}$ is the identity matrix of three dimensions.

Using equation $64, \vec{Y}$ can be obtained as:

$$
\vec{Y}=X / \gamma
$$

where

$$
\begin{gathered}
\gamma=\left(\lambda_{\max }+\frac{1}{2} \operatorname{tr}(S)\right) \alpha-\operatorname{det}(S) \\
X=Z\left(\alpha I+\left(\lambda_{\max }-\frac{1}{2} \operatorname{tr}(S)\right) S+S^{2}\right) \\
\alpha=\lambda_{\max }^{2}-\left(\frac{1}{2} \operatorname{tr}(S)^{2}\right)-\operatorname{tr}(\operatorname{adj}(S))
\end{gathered}
$$

The attitude quaternion is given by equation 67 .

$$
\bar{q}_{o p}=\frac{1}{\sqrt{\gamma^{2}+|X|^{2}}}\left[\begin{array}{l}
\gamma \\
X
\end{array}\right]
$$

It can be observed that in order to solve the equations above, $\lambda_{\max }$ needs to be computed. This value is computed by solving equation 68 .

$$
\lambda^{4}-(a+b) \lambda^{2}-c \lambda+\left(a b+\frac{c}{2} \operatorname{tr}(S)-d\right)
$$

where

$$
\begin{gathered}
a=\left(\frac{1}{2} \operatorname{tr}(S)\right)^{2}-\operatorname{tr}(\operatorname{adj}(S)) \\
b=\left(\frac{1}{2} \operatorname{tr}(S)\right)^{2}+Z^{T} Z \\
c=\operatorname{det}(S)+Z^{T} S Z \\
d=Z^{T} S^{2} Z
\end{gathered}
$$

To solve equation 67 , the author uses the iterative scheme Newton-Raphson method to obtain the value of $\lambda_{\max }$. Since the QUEST algorithm utilises iterative methods, it is considered to be less robust than the methods that were explained earlier. Furthermore, the $\vec{Y}$ is similar to the Gibbs vector but the author makes a point of not using the latter for defining the quaternion, because the Gibbs vector runs into singularity problems when the system is rotated by $180^{\circ}$.

Another similar method, ESOQ, developed by Mortari (1997), utilises the same scheme as QUEST to obtain the $\lambda_{\max }$ but has a slightly different approach to calculate the optimal quaternion. According to Markley and Mortari (2000) and Cheng and Shuster (2007a), out of the point-to-point attitude determination algorithms TRIAD, FOAM, SVD, QUEST, ESOQ and ESOQ2, the QUEST algorithm preforms the fastest in terms of execution time. The major benefit of these algorithms is that they are independent of the linearised body dynamics and are not required to be initialised. 


\subsection{REQUEST}

One of the shortcomings of the previously explained algorithms is that they are pure point-to-point algorithms, i.e., the algorithms obtain attitude of the system by using the simultaneously observed vectors (at least two vectors) in one time step. Due to this, the point-to-point algorithms do not utilise the system dynamics and the previously obtained observation data. This shortcoming is overcome by methods such as REQUEST (Bar-Itzhack (1996)) and Filter QUEST (Cheng and Shuster (2007b)).

The $Q$ matrix given in equation 47 is redefined as equation 69 .

$$
Q_{k}=\sum_{i=1}^{k} \frac{1}{\sigma^{2}} \vec{a}_{B_{i}}\left(\vec{a}_{I_{i}}\right)^{T}
$$

The term " $B_{k} "$ is calculated as shown in equation 70 .

$$
\begin{gathered}
Q_{0}=0 \\
Q_{k}=Q_{k-1}+\Delta Q_{k}, k=1 \ldots n
\end{gathered}
$$

where

$$
\Delta Q_{k}=\frac{1}{\sigma^{2}} \vec{a}_{B_{i}}\left(\vec{a}_{I_{i}}\right)^{T}, k=1 \ldots n
$$

If the system dynamics is changing in accordance with equation 71, then the matrix $Q$ must be developing in a similar way. Using the same logic, the associated co-variance matrices can be obtained as a function of $k$, as shown in equation 72 .

$$
\begin{gathered}
C_{k}=\Phi_{k-1} C_{k-1} \\
Q_{0,0}=0 \\
Q_{k, k-1}=\Phi Q_{k-1, k-1}, k=1, \ldots, n \\
Q_{k, k}=Q_{k, k-1}+\Delta Q_{k}
\end{gathered}
$$

where $Q$ is called attitude profile matrix.

If a priori information about the initial estimate $\left(C^{*}(-)\right)$ and the respective error co-variance matrix $\left(P_{0,0}(-)\right)$ is available, then the attitude profile matrix can be initialised as 73 .

$$
Q_{0,0}=\left[\frac{1}{2} \operatorname{tr}\left(P_{0,0}^{-1}(-)\right) I_{3 \times 3}-P_{0,0}^{-1}(-)\right] A^{*}(-)
$$

Furthermore, in order to include process noise, equation 72 is replaced by 74 , where $\alpha$ is the forgetting factor.

$$
Q_{k, k-1}=\alpha \Phi_{k-1} B_{k-1, k-1}, k=1, \ldots, n
$$

If the system model is simple, then the filter QUEST performance is similar to that of the Kalman filter, but for more pragmatic data, the estimation error of the Kalman filter outperforms the filter QUEST method.

An alternative to the filter $Q U E S T$ is the REQUEST algorithm, which emphasises on the propagation of Davenport's $K$ matrix (equation 75 instead of the propagation of the matrix $Q_{k}$.

$$
K_{k, k}=K_{k, k-1}+\Delta K_{k}, k=1, \ldots \ldots, n
$$


with

$$
\Delta K_{k}=\frac{1}{\sigma_{k}^{2}}\left[\begin{array}{cc}
\Delta S_{k}-\Delta S_{k} I_{3 \times 3} & \Delta z_{k} \\
\Delta Z_{k}^{T} & \Delta S_{k}
\end{array}\right]
$$

To obtain the quaternion transition matrix, the angular velocity is assumed to be constant $\left(\omega_{k}\right)$ for the incremental time step $\left(\delta t_{k}\right)$, and the quaternion transition matrix is obtained as shown in equation 76 .

$$
\bar{\Phi}_{k}=\exp \left(\operatorname{Omega}_{k} \Delta t_{k}\right)
$$

where

$$
\Omega_{k}=\frac{1}{2}\left[\begin{array}{cc}
-S\left(\omega_{k}\right) & \omega_{k} \\
-\omega_{k}^{T} & 0
\end{array}\right]
$$

After obtaining Davenport's $k$ matrix for each step, the REQUEST algorithm estimates the optimal quaternion using exactly the same eigenvalue-based method as the QUEST algorithm. Computationally, REQUEST is more expensive than Filter $Q U E S T$, but filter $Q U E S T$ is not adopted in many estimation processes because of its high estimation error compared to the Kalman filter. Both REQUEST and filter $Q U E S T$ have been shown to be mathematically equivalent by Shuster (2009) and Crassidis et al. (2007a). Furthermore, adaptive versions of REQUEST have been developed by Choukroun et al. (2004) and Choukroun (2007). Moreover, REQUEST and Filter $Q U E S T$ cannot estimate gyro biases, which is essential because they can be a significant source of error and need to be estimated especially where low-cost sensors are used in attitude estimation.

\subsection{Extended QUEST}

Wahba's cost function is only concerned with the attitude of the given systems. Thus, all the techniques that are trying to minimise the cost function only attain the attitude of the system. But in majority of the real time applications, along with the attitude of the system multiple other parameters need to be estimated. Thus, an extended version of Wahba's problem was proposed by Markley (1989) and Markley (1991), as shown in equation 77.

$$
L(R)=\frac{1}{2} \sum_{i=1}^{N} w_{i}\left|\vec{a}_{B_{i}}-R \vec{a}_{I_{i}}\right|^{2}+\frac{1}{2}\left(\beta-\beta^{-} W\left(\beta-\beta^{-}\right)\right)
$$

where $\beta^{-}$is the a priori parameter estimates information, $\beta$ is the estimated parameter, and $W$ is the weight matrix, which is semi-definite positive in nature and symmetric.

The extended QUEST algorithm presented by Psiaki (2000) falls in the class of algorithms that can extend the loss function to cater for the other states and parameters. The algorithm is build on the iterative $Q U E S T$ algorithm and utilises linearised system dynamics and square-root filtering techniques. The algorithm redefines Wahbas's loss function as equation 78 .

$$
\begin{gathered}
J=\frac{1}{2} \sum_{i=1}^{N} \frac{1}{\sigma_{i(k)}^{2}}\left|\vec{a}_{B_{i}}-R\left(\bar{q}_{k}\right) \vec{a}_{I_{i}}\right|^{2}+\frac{1}{2}\left|R_{\bar{q} \bar{q}_{k-1}}\left(\bar{q}_{k-1}-\hat{\bar{q}}_{k-1}\right)\right|^{2}+ \\
\frac{1}{2}\left|R_{w w_{k-1}} w_{k-1}\right|^{2}+\frac{1}{2}\left|R_{x \bar{q}_{k-1}}\left(\bar{q}_{k-1}-\hat{\bar{q}}_{k-1}\right)+R_{x x_{k-1}}\left(x_{k-1}-\hat{x}_{k-1}\right)\right|^{2}
\end{gathered}
$$

where $\bar{q}_{k}$ is the attitude quaternion, $x_{k}$ are the auxiliary states (additional states such as gyro bias, etc), $w_{k}$ is the process noise vector, $\hat{\bar{q}}_{k-1}$ is the estimated attitude quaternion at time step $k-1, \hat{x}_{k-1}$ is the estimated auxiliary state vector at time step $k-1, R_{x x_{k-1}}, R_{\bar{q} \bar{q}_{k-1}}, R_{x_{\bar{q}}-1}$ 
are the respective weight matrices and $R_{w w_{k-1}}$ is the random noise information matrix (standard deviation). Furthermore, the loss function is constrained by equation 79 .

$$
\begin{gathered}
\bar{q}=\frac{\bar{q}}{|\bar{q}|} \\
\bar{q}_{k}=\Phi\left(t_{k}, t_{k-1}, x_{k-1}, w_{k}\right) \bar{q}_{k-1} \\
x_{k}=h_{x}\left(t_{k}, t_{k-1}, \bar{q}_{k-1}, x_{k-1}, w_{k}\right)
\end{gathered}
$$

where $h$. is the auxiliary state transition function and $\Phi$ is the attitude transition function (kinematic and dynamic model of the system). The author assumes that the system noise is zero mean white noise. Equation 79 is used to obtain the attitude and auxiliary state prediction as given in equation 80 .

$$
\begin{gathered}
\overline{\bar{q}}_{k}=\Phi\left(t_{k}, t_{k-1}, \hat{x}_{k-1}, 0\right) \hat{\bar{q}}_{k-1} \\
\bar{x}_{k}=h_{x}\left(t_{k}, t_{k-1}, \hat{\bar{q}}_{k-1}, \hat{x}_{k-1}, 0\right)
\end{gathered}
$$

where $\overline{\bar{q}}_{k}$ and $\bar{x}_{k}$ are the predicted attitude quaternion and auxiliary state vectors respectively. The dynamic system equations are further linearised about the previous time step estimate (k-1).

The loss function given in equation 78 can be reformulated in terms of predicted values as shown in equation 81 .

$$
\begin{gathered}
J=\frac{1}{2} \sum_{i=1}^{N} \frac{1}{\sigma_{i(k)}^{2}}\left|\vec{a}_{B_{i}}-R\left(\bar{q}_{k}\right) \vec{a}_{I_{i}}\right|^{2}+\frac{1}{2}\left|R_{\bar{q} \bar{q}_{k}}\left(\bar{q}_{k}-\overline{\bar{q}}_{k}\right)\right|^{2}+ \\
\frac{1}{2}\left|R_{x \bar{q}_{k}}\left(\bar{q}_{k}-\overline{\bar{q}}_{k}\right)+R_{x x_{k}}\left(x_{k}-\bar{x}_{k-1}\right)\right|^{2}
\end{gathered}
$$

It is assumed that the auxiliary state do not effect the quaternion norm constraint. Thus, by taking the partial derivation of the loss function with respect to the auxiliary states and setting the derivative equal to zero, the optimal value of the auxiliary state vector is obtained as shown in equation 82 .

$$
x_{k_{o p t}}=\bar{x}_{k}-R_{x x_{k}}^{-1} R_{x \bar{q}_{k}}\left(\bar{q}_{k}-\overline{\bar{q}}_{k}\right)
$$

Substituting equation 82 and equation 63 in cost function 81 gives the loss function as shown in equation 83. The obtained function is minimised in a similar way to recursive QUEST to obtain the estimated quaternion $(\hat{\bar{q}})$. Since the estimated quaternion is the best available estimate of the attitude equation, the auxiliary state equation (82) gets updated to equation 84 .

$$
\begin{gathered}
J=\frac{1}{2} \bar{q}_{k}^{T} \sum_{i=1}^{N} \frac{1}{\sigma_{i(k)}^{2}} K_{i} \bar{q}_{k}+\frac{1}{2}\left|R_{\bar{q} \bar{q}_{k}}\left(\bar{q}_{k}-\overline{\bar{q}}_{k}\right)\right|^{2} \\
x_{k_{o p t}}=\bar{x}_{k}-R_{x x_{k}}^{-1} R_{x \bar{q}_{k}}\left(\hat{\bar{q}}_{k}-\overline{\bar{q}}_{k}\right)
\end{gathered}
$$

The more recently developed algorithm SOAR (Sequential Optimal Attitude Recursion, Christian and Lightsey (2010)) is also a static attitude determination algorithm and is based on recursive QUEST. It is highly similar to MEFK (Multiplicative Extended Kalman Filter). SOAR is computationally expensive, as multiple matrix inverse are required to be calculated. 


\subsection{Discussion}

Deterministic methods such as QUEST, SVD and others are designed to obtain the system's attitude when at least two simultaneously observable non-colinear vectors are available. These methods, that were initially developed to solve Wahba's problem, were only concerned with optimising for the attitude, without considering the dynamics of the system or any other parameters. Later, however, more advanced recursive methods such as SOAR and recursive QUEST were developed that are inclusive of other parameters. They are very similar to Kalman filters in structure and in computation cost. All these methods are optimisation-based and thus computationally heavy. Furthermore, they are highly sensitive to noise.

To determine the attitude of systems such as satellites, star trackers are used, which provide simultaneous observations of multiple non-colinear vectors. The star sensors are of very high quality, so their sensor and process noise is very low. Thus, the algorithms considered in this chapter work very well for satellites, but they are problematic for cheap systems such as micro-UAVs, which have low computational capabilities and very high system and sensor noise. Since this is the type of system under study, the algorithms considered in this chapter are not suitable for the attitude determination. 


\section{Complementary filters}

\section{Introduction}

Another widely used class of attitude estimation methods in the field of UAV is the complementary filter. The main advantage of complementary filters over the other methods is the lower computational cost. Complementary filter are in general computationally cheaper, compared to other attitude estimation methods. There are multiple types or variations of complementary filters available, but the main principle according to which all of them work is to combine the reading of multiple complementary sensors to obtain a better attitude estimate.

Complementary sensors are the sensors having complementary characteristics, such as one measuring relative information, while the other measures absolute information. For example, the accelerometer and rate gyroscope have complementary characteristics. The accelerometer measures the gravity vector along with a lot of noise, but the measurement is absolute in nature. It is not possible to trust the accelerometer reading alone, due to the reasons mentioned in section 2.3, but in a long-term view, the accelerometer does not drift off, as it is always measuring the gravity vector and the vehicle accelerations. The rate gyro measures the rotation rate that is integrated in order to obtain the attitude, but during this process the gyro bias also gets integrated and thus there is an error build-up over time. This error cannot be corrected by using only the gyro measurements. Thus, in order to compensate for each other's individual weaknesses, the two sensor are fused together using complementary filters.

Baerveldt and Klang (1997) present one of most basic complementary filters. The schematics are shown in figure 7.1. The filter utilises two sensors, namely an inclinometer and a rate gyroscope, to obtain a reading of the attitude of the system. The inclinometer gives the absolute inclination of the system, but the readings are filled with noise, usually of high frequency. Thus the inclinometer readings are filtered in such a way that the branch of the filter and the inclinometer in total act as a low-pass filter. On the other hand, the estimate done by the rate gyro is plagued by the gyro drift, thus the rate gyro readings are filtered in such a way that the branch of the rate gyro acts as a high-pass filter. This is where the name 'complementary filter' comes from.

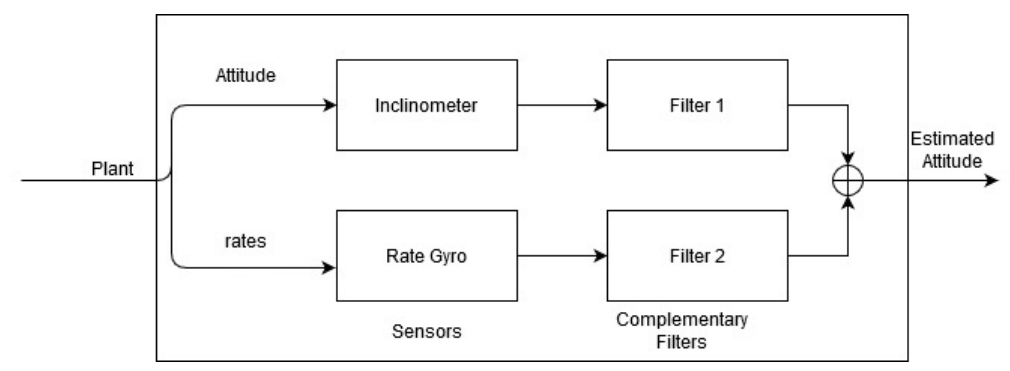

Figure 7.1: The schematic of the complementary filter by Baerveldt and Klang (1997)

The filters chosen by the author are given by equation 85 .

$$
\begin{aligned}
& F_{i}(s)=\frac{2 \tau s+1}{(\tau s+1)^{2}} \\
& F_{g}(s)=\frac{\tau s^{2}}{(\tau s+1)^{2}}
\end{aligned}
$$

where $F_{i}(s)$ is the filter for the inclinometer and $F_{g}(s)$ is the filter for the rate gyro. It can be observed that $F_{i}(s)+F_{g}(s)=1$. The tuning variable $\tau$ is obtained via the bode plot of each individual sensor.

Figure 7.2 shows another very simple realisation of a complementary filter (discrete form), where 
$t$ is the gain that is usually tuned depending upon the sensor noise.

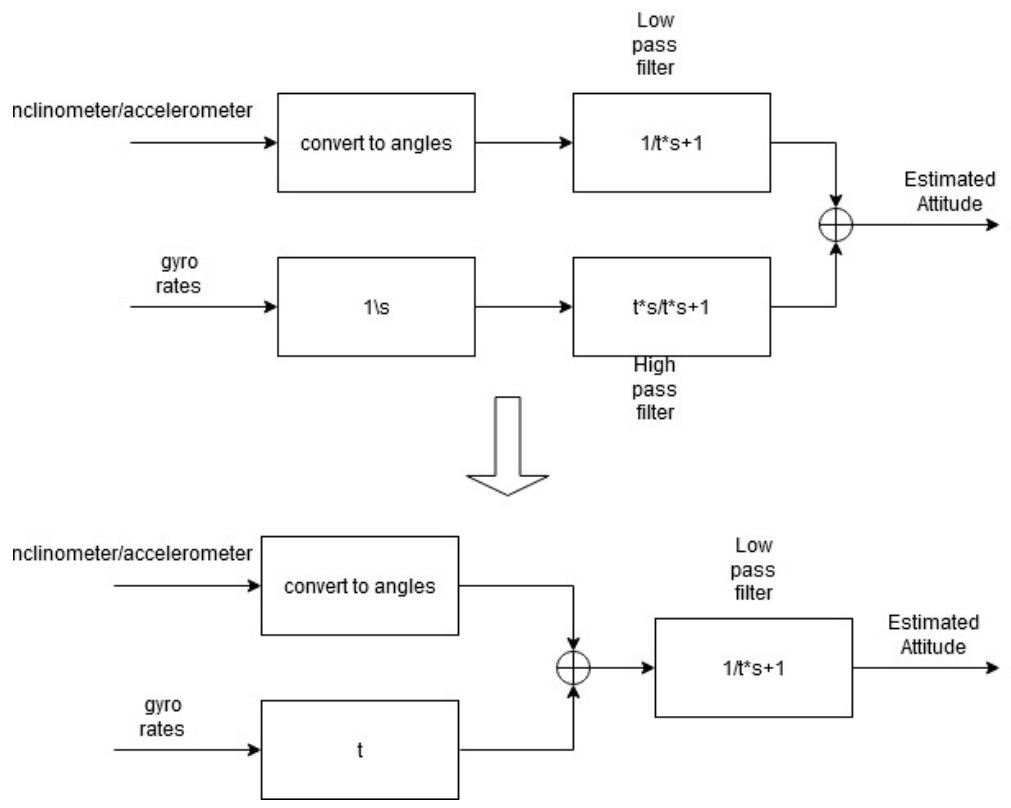

Figure 7.2: Realisation of a complementary filter

\subsection{Mahony Filter}

A complementary filter popularly known as Mahony filter (named after Mahony et al. (2008)) is one of the most often used complementary filters in the field of attitude estimation of a UAV. The author developed three complementary filters, namely the Direct complementary filter, Passive complementary filter and Explicit complementary filter. The most significant filter among the three filters is the Explicit complementary filter. All the three filters are based on the assumption that there exist at least two known non-co-linear vectors in the inertial frame and that the observation of these vectors is available in the body frame. The filter is given by equation 86 .

$$
\begin{gathered}
\dot{\hat{R}}=\hat{R}\left(\left(\omega_{\text {gyro }}-\hat{b}\right)+k_{p} \omega_{m}\right), \hat{R}(0)=\hat{R}_{0} \\
\dot{\hat{b}}=-k_{I} \omega_{m} \\
\omega_{m}=\sum_{i=1}^{n} k_{i} v_{i} \times \hat{v}_{i}
\end{gathered}
$$

where $\hat{v}_{i}:=R_{T} \vec{a}_{i}, \vec{a}_{i}$ is the known vector in the inertial frame, $\hat{v}_{i}$ is the estimated known vector in the body frame, $k_{I}$ is the positive integral gain, $k_{p}$ is the positive proportional gain and $k_{i}$ is the weight associated with the observed vector, which is usually set as unity or selected in such a way that matrix $M_{0}$ given by equation 87 is positive semi-definite.

$$
M_{0}=R_{T} k_{i} \vec{a}_{i} \vec{a}_{i}^{T}
$$

The matrix $M_{0}$ being semi-positive definite is required to prove the stability of the filter. Although the filter requires a minimum of two non-co-linear vectors to estimate the complete attitude of the system, under persistent excitation condition, i.e, when the direction of the observed vector is continuously changing, only one vector observation is enough for an orientation estimation, as shown by Mahony et al. (2009). Jensen (2011) posed a generalised form of the Mahony filter by replacing the positive proportional and integral with time-varying positive-definite gain matrix $K_{p}$ and $K_{I}$. Furthermore, the author proves that bias-free MEFK and constant-gain MEFK are special cases 
of the generalised form of the Mahony filter.

Although the Mahony filter is developed for the $S O(3)$ group (special orthogonal 3 group), it can easily be translated into quaternions, as shown in algorithm 1 .

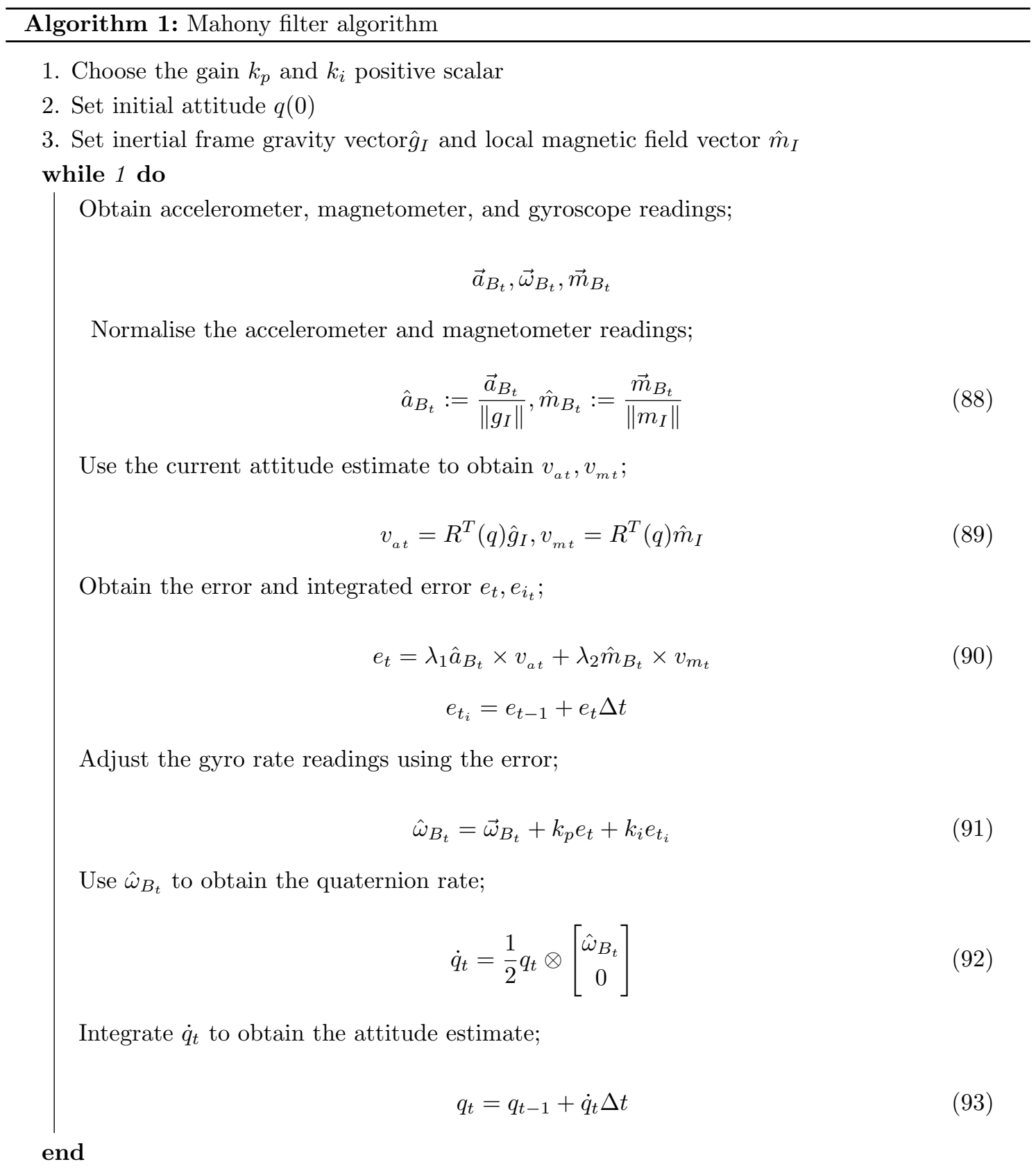

where $\vec{a}_{B_{t}}$ is the current accelerometer reading, $\vec{m}_{B_{t}}$ is the current magnetometer reading, $\vec{\omega}_{B_{t}}$ is the current rate gyroscope reading, $\vec{g}_{I}$ is the gravity vector in the inertial frame, $\vec{m}_{I}$ is the local earth magnetic vector in the inertial frame, $R_{B / I}$ is the rotation matrix, $\lambda_{1}$ is the weight for the accelerometer error, $\lambda_{2}$ is the weight for the magnetometer error (usually both are unity), $k_{p}$ is the error-proportional gain and $k_{i}$ is the integrated error gain.

The gains $\lambda_{1}$ and $\lambda_{2}$ are unity, provided the net external acceleration and magnetic distortion are close to zero. The gains $k_{p}$ and $k_{i}$ are required to be tuned by the user. The Mahony filter shoes that the gyro drift and bias can be compensated using a simple ordination filter through the PI (proportional integrator) feedback of the error in the rate of change of the attitude. The 
Mahony filter provides a significant improvement in the accuracy of the attitude estimation without a significant increase in the computational cost compared to the traditional complementary filter. This is evident from the paper by Jouybari et al. (2017). The approach assumes that the accelerometer readings are dominated by gravity and there is not much magnetic distortion. Figure 7.3 shows the schematics of the filter.

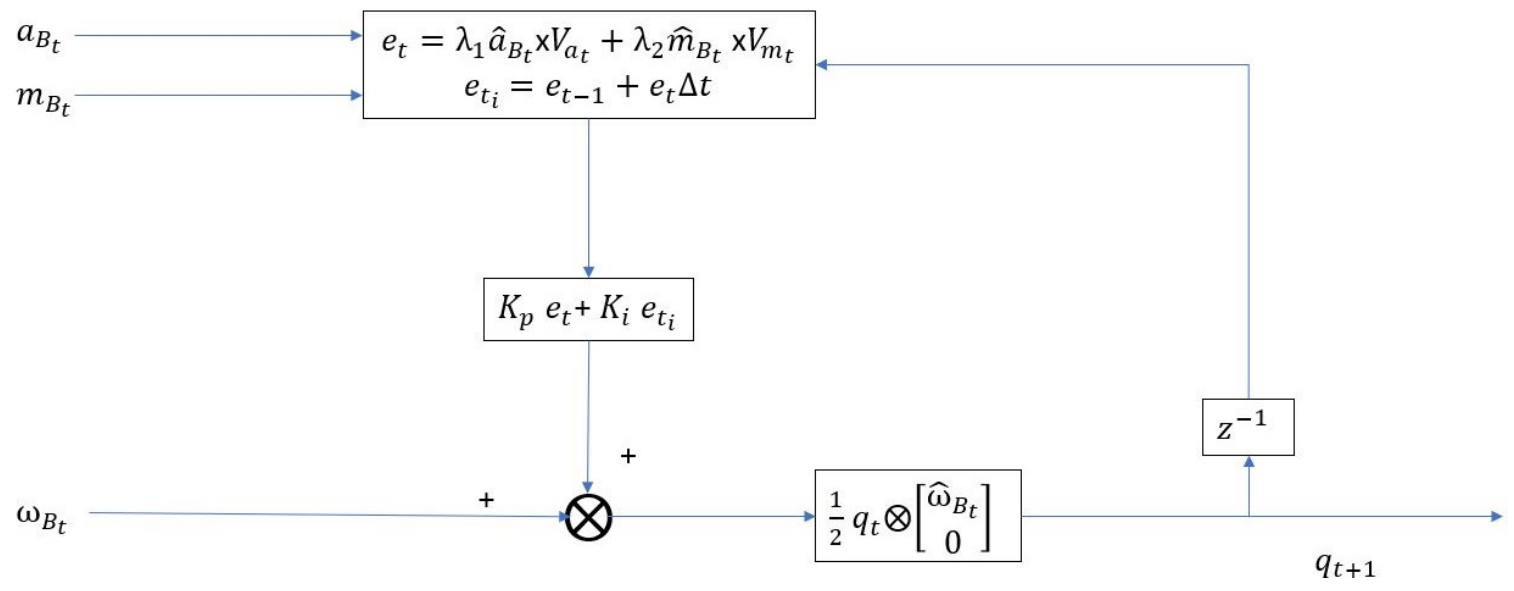

Figure 7.3: Mahony filter Schematics

\subsection{Madgwick filter}

Another famous complementary filter popularly known as Madgwick filter, developed by Madgwick et al. (2011) is used to address the problem of system attitude estimation at low computational cost. The author utilises quaternion representation of orientation to avoid the singularity problems encountered in the Euler angle representation. The principle of the filter is to obtain the attitude quaternion rate from the rate gyro readings and fuse the obtained quaternion rate with the one obtained via magnetometer and accelerometer. An optimisation algorithm is formulated to align the inertial frame vectors (gravity and local earth's magnetic field) with the sensor measurements (magnetometer and accelerometer readings) to obtain the attitude quaternion rate. The author uses gradient-descent algorithm for optimisation. Furthermore, the gyroscope drift bias is corrected by an orientation filter through the integral feedback of the error in the gyroscope readings.

The quaternion rate is obtained as shown in equation 94.

$$
\dot{q}_{t_{\omega}}=\frac{1}{2} \hat{q}_{t-1} \otimes \omega_{t}
$$

where $\dot{q}_{t}$ is the quaternion rate, $\hat{q}_{t-1}$ is the previous time-step attitude estimate and $\omega_{t}$ is the current rate gyro reading.

The optimisation algorithm used in the development of the filter, i.e., the gradient descent algorithm, is depicted in equation 95. Gradient descent is an iterative optimisation algorithm which minimises the function by moving in steepest descent, defined by the gradient. There are multiple versions of gradient descent, the most widely known versions are general gradient descent, such as stochastic gradient descent and mini-batch gradient descent. The method used in the paper by Madgwick is the general gradient descent.

$$
\begin{gathered}
\theta_{j}:=\theta_{j}-\alpha \frac{\partial J(\bar{\theta})}{\partial \theta_{j}}, j=1,2, \ldots ., n \\
\bar{\theta}=\theta_{0}, \theta_{1} \ldots \ldots, \theta_{n}
\end{gathered}
$$

Using the gradient descent algorithm, the Madgwick filter is given as shown in equation 96 . 


$$
\begin{aligned}
& \dot{\hat{q}}_{t}=\dot{q}_{t_{\omega}}+\beta \dot{q}_{t_{s}} \\
& \dot{q}_{t_{s}}=\frac{\Delta f}{\|\Delta f\|} \\
& \Delta f=\left\{\begin{array}{c}
J_{g}^{T}\left(\hat{q}_{t-1}\right) f_{g}\left(\hat{q}_{t-1}, \hat{a}_{t}\right) \\
J_{b}^{T}\left(\hat{q}_{t-1}, \hat{b}\right) f_{b}\left(\hat{q}_{t-1}, \hat{b}, \hat{m}_{t}\right)
\end{array}\right\} \\
& f_{g}(\hat{q}, \hat{a})=\left[\begin{array}{l}
2\left(q_{2} q_{4}-q_{1} q_{3}\right)-a_{x} \\
2\left(q_{1} q_{2}+q_{3} q_{4}\right)-a_{y} \\
2\left(\frac{1}{2}-q_{2}^{2}-q_{3}^{2}\right)-a_{z}
\end{array}\right] \\
& J_{g}(\hat{q})=\left[\begin{array}{cccc}
-2 q_{3} & 2 q_{3} & -2 q_{1} & 2 q_{2} \\
2 q_{2} & 2 q_{1} & 2 q_{4} & 2 q_{3} \\
0 & -4 q_{2} & -4 q_{3} & 0
\end{array}\right] \\
& f_{b}(\hat{q}, \hat{b}, \hat{m})=\left[\begin{array}{c}
\left.2 b_{x}\left(0.5-q_{3}^{2}-q_{4}^{2}\right)+2 b_{z}\left(q_{2} q_{4}-q_{1} q_{3}\right)-m_{x}\right) \\
2 b_{x}\left(q_{2} q_{3}-q_{1} q_{2}\right)+2 b_{z}\left(q_{1} q_{2}+q_{3} q_{4}\right)-m_{y} \\
2 b_{x}\left(q_{1} q_{3}+q_{2} q_{4}\right)+2 b_{z}\left(0.5-q_{2}^{2}-q_{3}^{2}\right)-m_{z}
\end{array}\right] \\
& J_{b}(\hat{q}, \hat{b})=\left[\begin{array}{cccc}
-2 b_{z} q_{4} & 2 b_{z} q_{4} & -4 b_{z} q_{3}-2 b_{z} q_{1} & -4 b_{x} q_{4}+2 b_{z} q_{2} \\
-2 b_{x} q_{4}+2 b_{z} q_{2} & 2 b_{x} q_{3}+2 b_{z} q_{1} & 2 b_{x} q_{2}+2 b_{z} q_{4} & -2 b_{x} q_{1}+2 b_{z} q_{3} \\
2 b_{x} q_{3} & 2 b_{x} q_{4}-4 b_{z} q_{2} & 2 b_{x} q_{1}-4 b_{z} q_{3} & 2 b_{x} q_{2}
\end{array}\right]
\end{aligned}
$$

where $\beta$ is the constant gain, $q$ is the attitude quaternion, $\hat{a}_{t}$ is the normalised accelerometer reading, $\hat{b}_{t}$ is the normalised local earth's magnetic field, $\hat{h}_{t}$ is the normalised magnetometer reading, $\dot{q}_{t_{\omega}}$ is the rate gyro reading and $\dot{\hat{q}}_{t}$ is the estimated quaternion rate that is integrated to obtain the system's attitude.

The magnetometer readings can suffer from distortions because of ferromagnetic material. This can lead to two types of errors: Horizontal plane error, i.e., the error in the magnetometer readings along the plane horizontal to the earth surface (declination error), which cannot be corrected without another reference vector. The other error is the vertical plane error, which can be corrected, as the accelerometer readings provide an additional vector (assuming that the accelerometer measures only gravity). The author computes the earth's magnetic field vector $\left(\hat{b}_{t}\right)$ as shown in equation 97. Due to this, the local earth's magnetic field is represented only along the $x$ and $z$ inertial axes.

$$
\begin{gathered}
\hat{b}=\left[\begin{array}{llll}
0 & b_{x} & 0 & b_{z}
\end{array}\right] \\
\hat{m}=\left[\begin{array}{llll}
0 & m_{x} & m_{y} & m_{z}
\end{array}\right]
\end{gathered}
$$

Since the earth's gravity is assumed to be along the $x$ axis of the inertial frame, the inclination error can be compensated. Thus the local magnetic field's distortion does not effect the pitch and roll estimated. Furthermore, in order to cater for the gyro bias drift, the gyro readings are corrected using an orientation filter through an integral feedback as shown in equation 98.

$$
\omega_{c}=\omega_{t}-\omega_{b}
$$




$$
\begin{aligned}
& \omega_{b}=\tau \sum_{t} \omega_{e} \Delta t \\
& \omega_{e}=2 q_{t-1} \otimes \dot{q}_{t_{s}}
\end{aligned}
$$

where $\omega_{c}$ is the omega corrected and $\tau$ is the integral tuning gain. The algorithm for the Madgwick filter is given in algorithm 2. Figure 7.4 gives the Madgwick filter schematics.

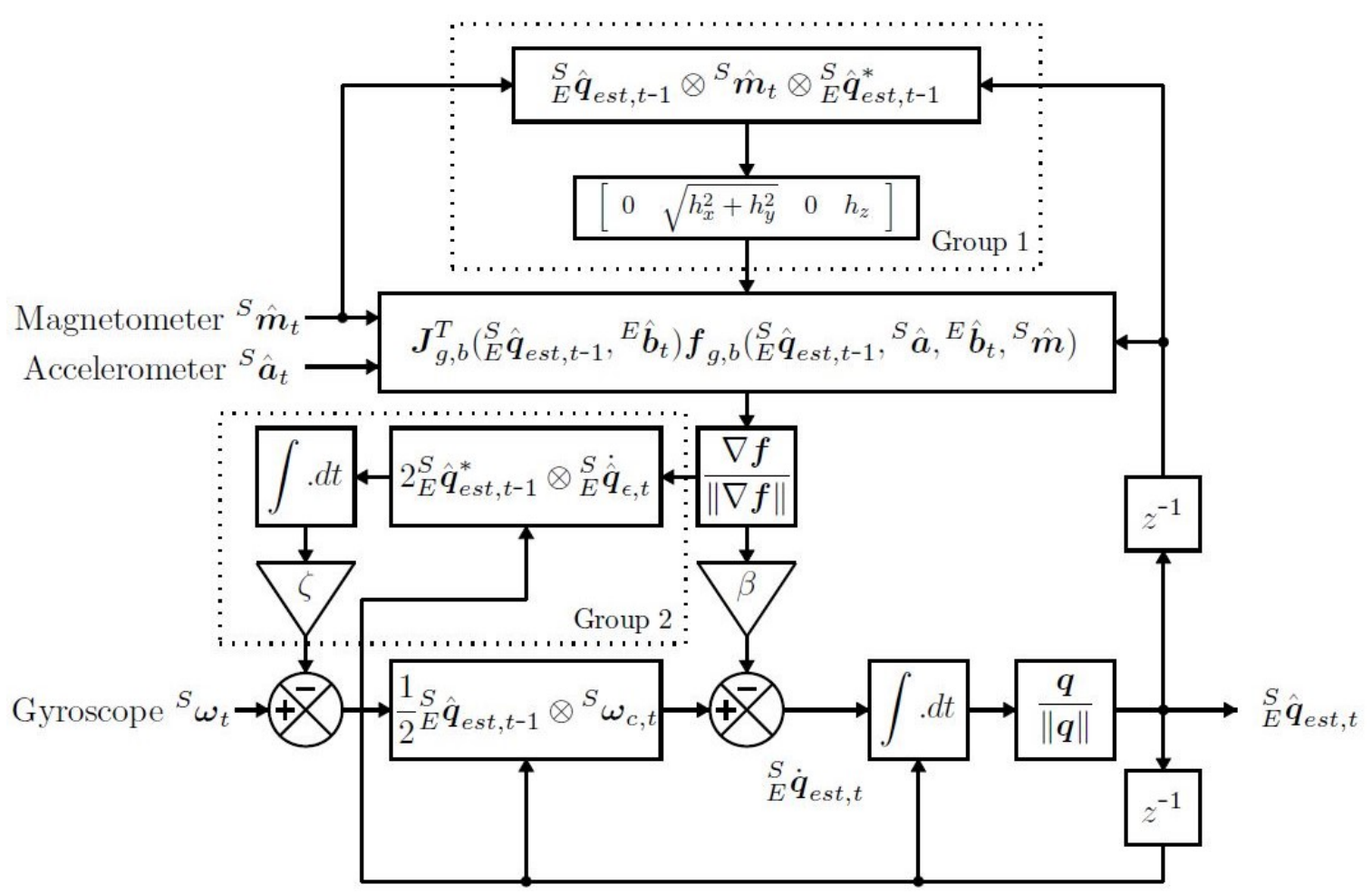

Figure 7.4: Madgwick filter Schematics (Madgwick et al. (2011)) 


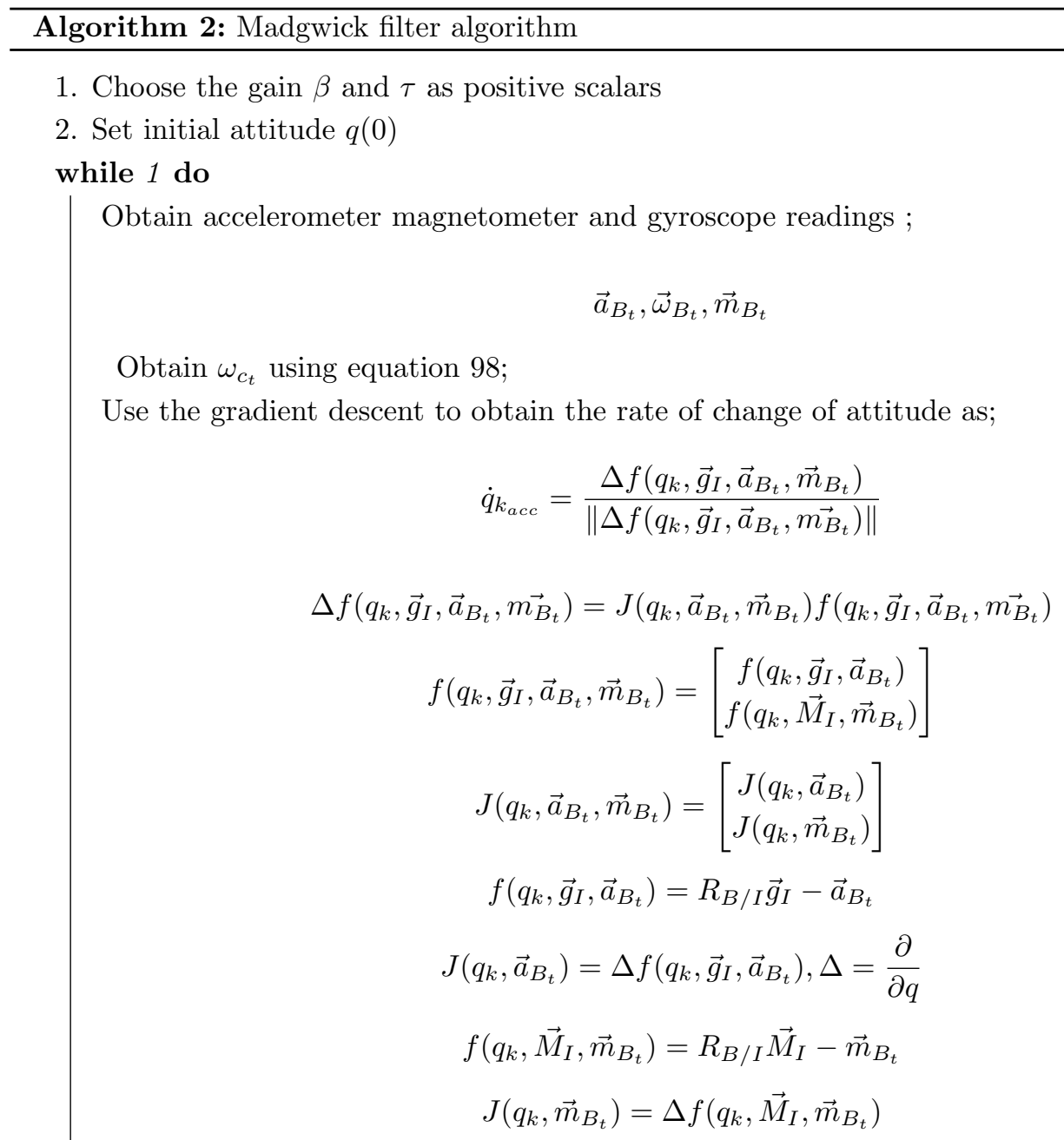

Obtain the quaternion rate using gyro readings and previous optimal attitude estimate;

$$
\dot{q}_{k_{\text {gyro }}}=\frac{1}{2} q_{k-1} \otimes \omega_{c_{t}}
$$

Obtain the optimal estimated quaternion rate ;

$$
\dot{q}_{k_{e s t}}=\dot{q}_{k_{g y r o}}+\beta \dot{q}_{k_{a c c}}
$$

Integrate the obtained rate to get the attitude;

$$
q_{k}=q_{k-1}+\dot{q}_{k_{e s t}} d t
$$

Normalize the quaternion

$$
q_{k}=\frac{q_{k}}{\left\|q_{k}\right\|}
$$

end

where $\vec{a}_{B_{t}}, \vec{m}_{B_{t}}, \vec{\omega}_{B_{t}}$ are the accelerometer, magnetometer and rate gyro current readings, $R_{B / I}$ is the rotation matrix (eq:23), $\vec{g}_{I}$ is the gravitation vector in the inertial frame, and $\vec{M}_{I}$ is the local magnetic field vector in the inertial frame. The tuning gains $\beta$ and $\tau$ are either tunes or can be 
obtained using equation 104.

$$
\begin{gathered}
\beta=\left\|\frac{1}{2} \otimes\left[\begin{array}{llll}
0 & \tilde{\omega}_{\beta} & \tilde{\omega}_{\beta} & \tilde{\omega}_{\beta}
\end{array}\right]\right\|=\sqrt{\frac{3}{4}} \tilde{\omega}_{\beta} \\
\tau=\sqrt{\frac{3}{4}} \tilde{\dot{\omega}}_{\tau}
\end{gathered}
$$

where $\tilde{\omega}_{\beta}$ is the estimated zero mean gyroscope error and $\tilde{\dot{\omega}}_{\tau}$ is the gyro drift bias rate.

The Madgwick filter performs better than the simple complementary filter and the Mahony filter, with a a negligible increase in the computation cost, as shown by Ludwig et al. (2018). Furthermore, Ludwig and Burnham (2018) compare Madgwick, Mahony and the Extended Kalman filter performance on the quadcopter raw sensor data to estimate the attitude, and show that all the three filters obtain similar accuracy while the computation cost of Mahony is lowest, followed by the Madgwick filter (albeit being nearly the same as the Mahony filter), and EKF being the most computationally heavy.

Another paper (Cavallo et al. (2014)) compares the three filters and shows that in terms of accuracy, EKF performs the best, while the other two filters show similar results. In terms of computation, the results are similar to the previous comparison (Ludwig and Burnham (2018)). The difference in the results might be due to the formulation of the Kalman filter in the two papers, but the comparison of the other two filters (Mahony and Madgwick) shows the same results. Tables 7.1 and 7.2 summarise the results of the papers (1 indicating a higher performance than 2 ).

\begin{tabular}{|l|l|l|l|}
\hline Performance Rating & Ludwig et al. (2018) & Ludwig and Burnham (2018) & Cavallo et al. (2014) \\
\hline EKF & - & 1 & 1 \\
\hline Madgwick & 1 & 1 & 2 \\
\hline Mahony & 1 & 1 & 2 \\
\hline Complementary & 2 & - & - \\
\hline
\end{tabular}

Table 7.1: Performance comparison

\begin{tabular}{|l|l|l|l|}
\hline Computational Cost & Ludwig et al. (2018) & Ludwig and Burnham (2018) & Cavallo et al. (2014) \\
\hline EKF & - & 2 & 2 \\
\hline Madgwick & 1 & 1 & 1 \\
\hline Mahony & 1 & 1 & 1 \\
\hline Complementary & 2 & - & - \\
\hline
\end{tabular}

Table 7.2: Computational cost comparison

Although the Madgwick filter shows highly satisfactory results, there are a few drawbacks of the filter. It is assumed that the accelerometer measurements are dominated by the gravity vector and the local magnetic field does not vary with time. Thus the filter estimates deteriorate if the system experiences a prolonged high magnitude of external acceleration or there is a magnetic field disturbance.

A complementary filter that is very much identical to the Madgwick filter (Madgwick et al. (2011)) is presented by Tian et al. (2013). The main difference between both the filters is the algorithm used to obtain the attitude quaternion from the magnetometer and accelerometer readings. In the Madgwick filter, the gradient descent algorithm is used, while Tian et al. (2013) uses the GaussNewton algorithm as the minimisation scheme. The part that the author claims to be adaptive is not actually adaptive in nature, since the fusion gain stays constant. The author argues that the Gauss-Newton algorithm has a better convergence than the gradient descent algorithm, but it requires more computational power. According to the author, it is acceptable to have one iteration per time-step by the Gauss-newton algorithm, as long as the converge rate, which is governed by 
a constant, is equal or grater than the physical rate of change of the state. Figure 7.5 shows the schematics of the filter. Al-Fahoum and Abadir (2018) wrote a similar paper, in which the the gradient descent algorithm is replaced by an optimized Lavenberg-Marquardt algorithm.

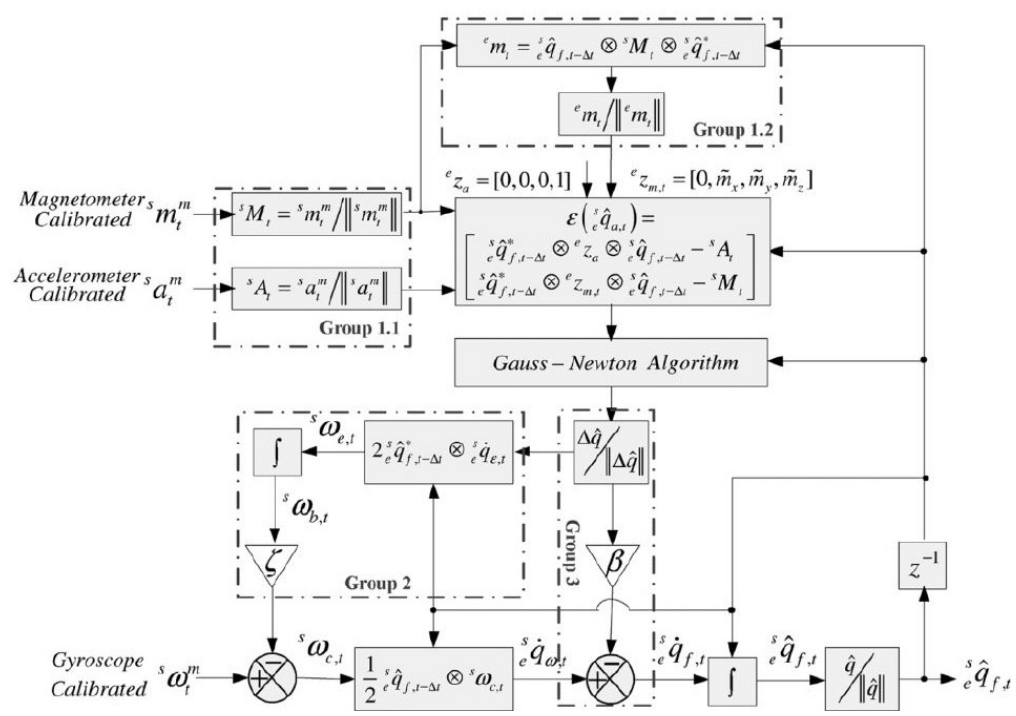

Figure 7.5: Filter schematics (Tian et al. (2013))

\subsection{Adaptive-gain complementary filter}

Calusdian et al. (2011) presents another adaptive-gain complementary filter. The schematics of the filter are shown in figure 7.6. The filter is very straightforward. It utilises the Factored quaternion algorithm to obtain the current attitude quaternion through the magnetometer and accelerometer readings. Then the previous time-step optimum estimates are subtracted from the obtained estimate (from the magnetometer and accelerometer readings) to obtain an error. The obtained error is multiplied to a static gain and then summed to the rate quaternion obtained from the rate gyro (using equation 29). Then the estimated rate is integrated to obtain the estimated attitude for the current time step.

Calusdian provides an insight that the methods such as QUEST and FAQ can be used in determining the attitude, but the paper has several problems. The author assumes that the accelerometer readings are dominated by the gravity vector and there is no magnetic distortion. Furthermore, the problem of sensor time-varying bias has not been addressed. The author furthermore estimates the quaternion error by just subtracting the quaternions, which is a wrong, as the quaternion error should be obtained via equation 20. Furthermore, as stated in chapter 6, point-to-point methods such as FQA and QUEST are very sensitive to system noise and thus not suitable for low-cost sensors that are used in UAVs.

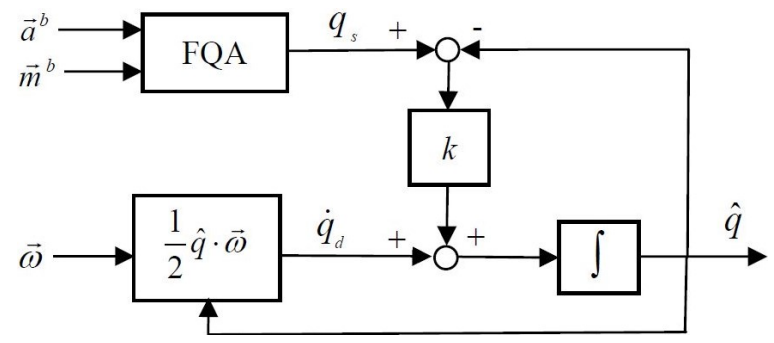

Figure 7.6: Adaptive-gain complementary filter schematics (Calusdian et al. (2011))

Another paper by Marantos et al. (2016) presents a different approach to fuse the obtained sensor readings. The filter utilises SVD (one of the Wahba's optimisation algorithms) to find the 
fused estimates. Wahba's optimisation algorithm needs two different observation vectors in order to estimate the attitude in the least square sense, which are obtained via the magnetometer and the accelerometer. But to filter out the sensor noise, local magnetic field distortion and external accelerations, gyroscope measurements are utilised. The gyroscope data is propagated through a set of reference vectors (local gravity vector and local magnetic field vector). These propagated measurements are added to the respective sensor measurements (magnetometer and accelerometer) along with a weight. The weight is adaptive in nature and depends on the error between the constant angle (between the local gravity vector and the local magnetic field vector) and the disturbed angle. Furthermore, the weight also depends on the error between the sensor measurements (magnetometer and accelerometer) and the gyroscope's propagated measurements. The weight for the accelerometer part is given by equation 105 .

$$
w_{a, k}=w_{a, 0}\left(1-\frac{c_{3} e_{a}^{k}}{\sqrt{1+\left(c_{3} e_{a}^{k}\right)^{2}}}\right)
$$

where

$$
\begin{gathered}
e_{a}^{k}=e_{\theta}^{k} \cdot e_{n a}^{k} \\
e_{\theta}^{k}=\left|a_{B} \cdot m_{B}-a_{I} \cdot m_{I}\right| \\
e_{n a}^{k}=c_{1}\left\|a_{b}-R a_{I}\right\|+c_{2}\left(\left\|a_{B}\right\|-\left\|a_{I}\right\|\right)
\end{gathered}
$$

where $w_{a, 0}$ is the undisturbed weight, $a_{B}$ is the accelerometer measurement, $m_{B}$ is the magnetometer measurement, $a_{I}$ is the local gravity vector and $m_{I}$ is the local magnetic field vector. $c_{*}$ are the gains with condition $c_{1}+c_{2}=1$ and $c_{1}, c_{2}>0$. Figure 7.7 gives the schematics of the filter.

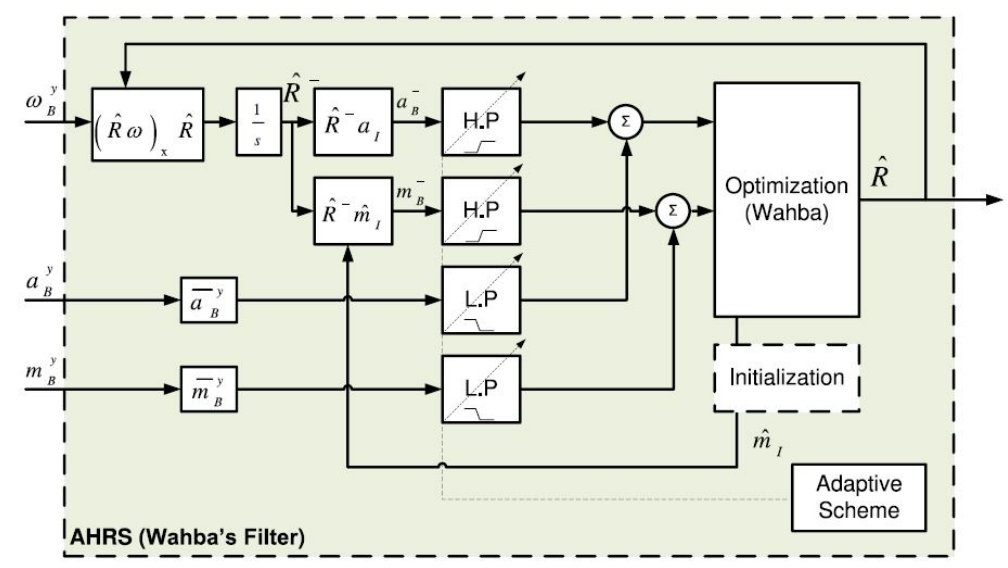

Figure 7.7: Adaptive filter by Marantos et al. (2016)

\subsection{Geometrically intuitive complementary filter}

Another recent complementary filter by Del Rosario et al. (2016) introduces a geometrically intuitive approach to fuse the sensor measurements. This approach is also based on quaternion representation, as it does not suffer from singularity problems. The filter is designed to obtain the attitude of the system by integrating gyro readings and then project the accelerometer readings in the inertial frame using the obtained attitude, so that the error between the projected accelerometer readings and the gravity vector is calculated. The attitude obtained from the gyro is further corrected by rotating it about the axis obtained from the cross product of the projected accelerometer readings and the gravity vector. The magnitude of rotation is equal to the obtained error multiplied by a scaling constant. The new attitude estimate is obtained. This attitude estimate is further corrected using magnetometer readings in the same manner to obtain the final attitude estimation.

The author has used the Madgwick filter explained above as a benchmark to test the filter. The 
filter assumes that the accelerometer readings are dominated by the gravity vector, hence if the system experiences prolonged external acceleration, the estimates worsen. The filter is more capable of handling magnetic distortion compared to the Madgwick filter. The filter also has a higher accuracy compared to the Madgwick filter in the presence of short-term magnetic distortion, as the filter has two separate correction loops which allow it to respond to the measurements from the magnetometer and accelerometer independently, thus decoupling the correction of the roll and pitch from the correction of the yaw. The filter performs marginally better than the Madgwick filter in terms of computational cost. The approach assumes that the system acceleration will average out to be zero over time, implying that accelerometer readings average out to be dominated mainly by the gravity vector and that the magnetometer does not suffer from magnetic distortion for an extended period of time. The schematics of the filter are given in figure 7.8. Algorithm 3 provides the algorithm of the filter.

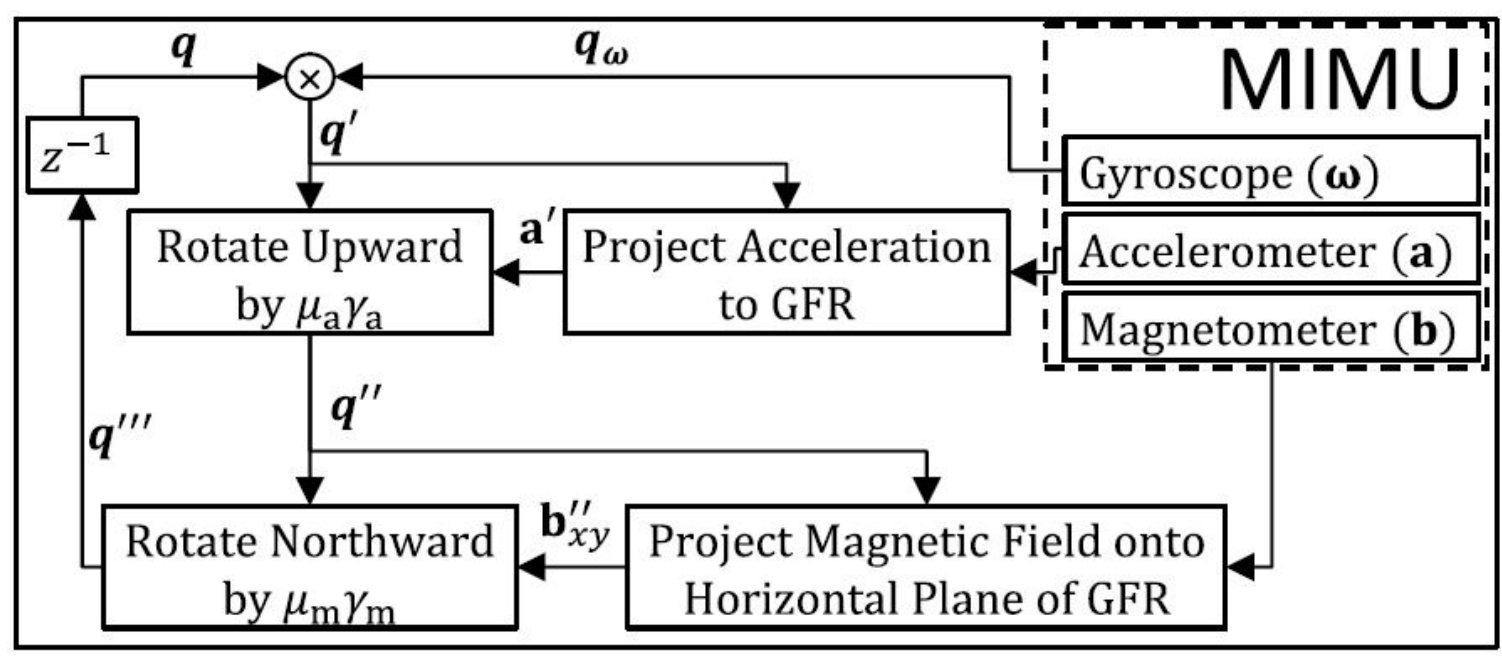

Figure 7.8: Geometrically intuitive approach complementary filter schematics (Del Rosario et al. (2016)) 


Algorithm 3: Geometrically intuitive complementary filter algorithm

1. Choose the gain $\mu_{a}$ and $\mu_{m}$ as positive scalars

2. Set the local magnetic field vector $e_{m}$

3. Set initial attitude $q(0)$

\section{while 1 do}

Obtain accelerometer magnetometer and gyroscope readings ;

$$
\vec{a}_{t}, \vec{\omega}_{t}, \vec{m}_{t}
$$

Obtain the current attitude quaternion using the gyro reading and previous attitude estimate

$$
\begin{gathered}
\dot{q}_{t}=\frac{1}{2} q_{t-1} \otimes\left[\begin{array}{ll}
\vec{\omega}_{t} & 0
\end{array}\right] \\
q_{t}^{\prime}=q_{t-1}+\dot{q}_{t} \Delta t
\end{gathered}
$$

Improve the estimate by projecting the accelerometer readings;

$$
\begin{gathered}
q_{t}^{\prime \prime}=q_{u p} \otimes q_{t}^{\prime} \\
q_{u p}=\left[\begin{array}{cc}
\cos \left(\frac{\mu_{a} \gamma_{a}}{2}\right) n_{u p} \sin \left(\frac{\mu_{a} \gamma_{a}}{2}\right) & 0
\end{array}\right] \\
n_{u p}=\vec{a}_{t}^{\prime} \times e_{u p} \\
\gamma_{a}=\cos ^{-1}\left(\frac{a_{t}^{\prime} \cdot e_{u p}}{\left\|a_{t}^{\prime}\right\|}\right) \\
{\left[\begin{array}{ll}
\vec{a}_{t}^{\prime} & 0
\end{array}\right]=q_{t}^{\prime} \otimes\left[\begin{array}{ll}
\vec{a}_{t} & 0
\end{array}\right] \otimes\left(q_{t}^{\prime}\right)^{*}} \\
e_{u p}=\left[\begin{array}{lll}
0 & 0 & 1
\end{array}\right]
\end{gathered}
$$

Further improve the reading by projecting the magnetometer readings;

$$
\begin{gathered}
q_{t}^{\prime \prime \prime}=q_{m} \otimes q_{t}^{\prime \prime} \\
q_{m}=\left[\begin{array}{cc}
\cos \left(\frac{\mu_{m} \gamma_{m}}{2}\right) n_{m} \sin \left(\frac{\mu_{m} \gamma_{m}}{2}\right) & 0
\end{array}\right] \\
n_{m}=\vec{m}_{t}^{\prime} \times e_{m} \\
\gamma_{m}=\cos ^{-1}\left(\frac{m_{t}^{\prime} \cdot e_{m}}{\left\|m_{t}^{\prime}\right\|}\right) \\
{\left[\begin{array}{ll}
\vec{m}_{t}^{\prime} & 0
\end{array}\right]=q_{t}^{\prime \prime} \otimes\left[\begin{array}{ll}
\vec{m}_{t} & 0
\end{array}\right] \otimes\left(q_{t}^{\prime \prime}\right)^{*}}
\end{gathered}
$$

Normalise the quaternion to obtain the best attitude estimate

$$
q_{t}=\frac{q_{t}^{\prime \prime \prime}}{\left\|q_{t}^{\prime \prime \prime}\right\|}
$$

\section{end}

The tuning parameters $\mu_{a}$ and $\mu_{m}$ are tuned depending upon the accuracy of the respective sensor and the gyro accuracy. The author assumes that the acceleration of the system and the magnetic interference will average out to be zero over time. 


\subsection{Fast complementary filter}

Another complementary filter by Wu et al. (2016) called 'fast complementary filter' uses the accelerometer readings and the gyroscope readings to obtain the estimates for roll and pitch of the system. In case there is no magnetic distortion, the magnetometer readings are fused with the obtained estimates using Markley's algorithm (one of the solution to Wahba's problem, SVD method) to correct for the yaw. Figure 7.9 shows the schematics of the filter. This approach prevents the local magnetic distortion from interfering with the roll and pitch estimates. The filter assumes that the accelerometer readings are dominated by the gravity vector which leads to wrong roll and pitch estimates if the system is projected to prolonged accelerations. Furthermore, in absence of magnetic distortion, the filter shows reduced accuracy compared to the Kalman filter-based methods, but has a lower computational cost compared to the Kalman filter-based methods. The filter has a better accuracy than the Madgwick filter in the presence of short-term magnetic distortion due to the separate correction of yaw.

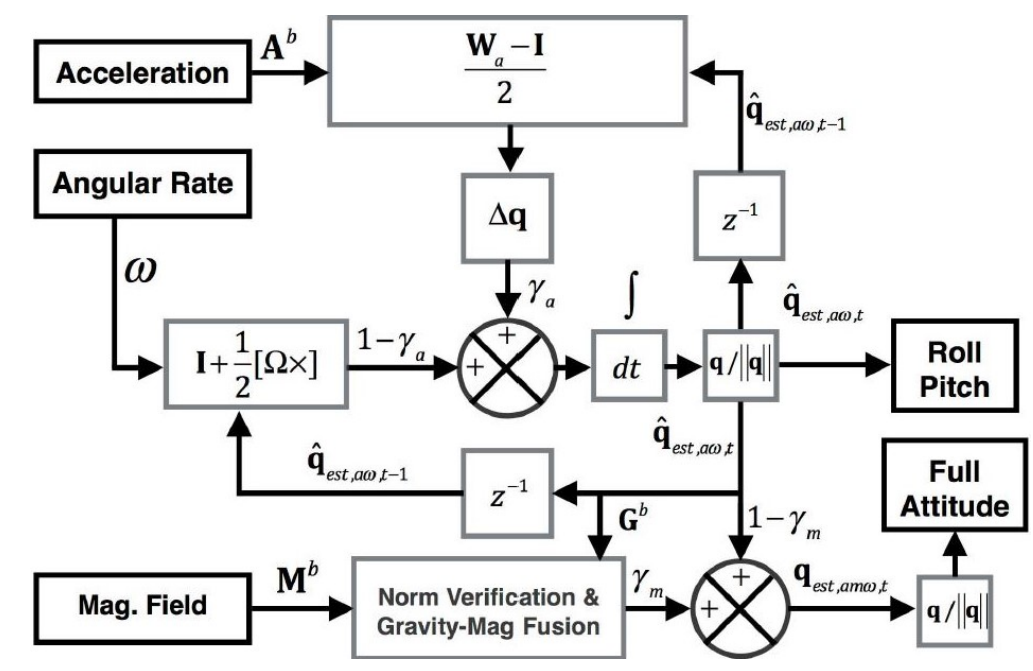

Figure 7.9: Fast complementary filter schematics (Wu et al. (2016))

\subsection{Discussion}

In this chapter, the most widely used complementary filters approaches were introduced and their strength and weaknesses have been discussed. The major advantage of complementary filters over the deterministic method is the complementary filters' ability to deal with sensor noise. Moreover, these filters operate on a very low computational cost compared to Kalman filters and are very easy to implement. But all the complementary filters rely on a few basic assumptions. Firstly, it is assumed that the accelerometer measurements are dominated by gravity measurements, i.e., the system's external accelerations are either small, short-lived or average out be zero. Under normal operation of the quadcopter, this assumption is valid, but in case of a failed rotor, the validity of the assumption is under doubt.

Secondly, most of the methods assume that in outdoor flights, there is not much local magnetic field distortion. Thus, in case there is actually a distortion in the local magnetic field, the estimates made by the filters will be effected. Some filters such as Madgwick et al. (2011) and Wu et al. (2016) represent the local magnetic field vector in such a way that if there is magnetic disturbance, then it only effects the yaw estimate and does not effect the roll and pitch estimate. It can be be observed that the estimation algorithm such as by Marantos et al. (2016) and Calusdian et al. (2011) utilises the previously explained deterministic methods as a part of the complete filter. Moreover, it can be seen that the filter developed by Madgwick et al. (2011) shows the best performance while having a relatively low computational cost. 


\section{Stochastic Estimation}

\section{Introduction}

Stochastic estimation is a type of estimation technique that is used to estimate the probability distribution function of a variable (system states) based on one or more observations of the system output. Stochastic estimation is primarily based on the Bayesian filter, which is a general layout for stochastic estimation filters. This chapter begins with a brief description of Bayesian filters. Then the most widely known forms of Bayesian filter, Kalman filter and its nonlinear version, EKF, are explained along with their respective shortcomings. Sussequently, a more sophisticated extension of the Kalman filter, namely the unscented Kalman filter (UKF), is explained briefly. Kalman Filter, EKF and UKF form the base of various attitude estimation techniques that are explored in the subsequent part of this chapter. They will be evaluated based on their usefulness for the purpose of this research, i.e., the application for state estimation in a quadcoptor subjected to rotor failure.

\subsection{Bayesian Filters}

In estimation theory, the Bayesian approach has the ability to include the prior information (already available information) about the system or the distribution in the estimation process. In Bayesian estimation, the primary goal is to estimate the system states $\left(x_{t}\right)$ given observations $\left(z_{1: t}\right)$, and control $\left(u_{1: t}\right)$ given to the system in a recursive manner. The Bayesian filters make the following primary assumptions:

- The first Markov assumption:

$$
p\left(y_{n} \mid x_{0: n}\right)=p\left(y_{n} \mid x_{n}\right)
$$

This implies that if we know the states at time $n$, then no additional information is obtained from the previous states or the previous input.

- The second Markov assumption: commands executed in future do not effect the current state of the system

According to Bayes theorem (110)

$$
p(x, y)=p(x \mid y) p(y)=p(y \mid x) p(x)
$$

The belief of $x_{t}$ is defined as

$$
\left.\operatorname{bel}\left(x_{t}\right)=p\left(x_{(} t\right) \mid z_{1: t}, u_{1: t}\right)
$$

using Bayes theorem

$$
\operatorname{bel}\left(x_{t}\right)=p\left(z_{t} \mid x_{t}, z_{1: t-1}, u_{1: t}\right) p\left(x_{t} \mid z_{1: t-1}, u_{1: t}\right)
$$

Then, according to Markov, the first assumption equation 112 can be written as equation 113.

$$
=p\left(z_{t} \mid x_{t}\right) p\left(x_{t} \mid z_{1: t-1}, u_{1: t}\right)
$$

The law of total probability states that (114)

$$
P(A)=\int_{B} P(A \mid B) P(B) \mathrm{d} B
$$


By using the law of total probability equation, 113 can be written as equation 115 .

$$
=p\left(z_{t} \mid x_{t}\right) \int_{x_{t-1}} p\left(x_{t} \mid x_{t-1}, z_{1: t-1}, u_{1: t}\right) p\left(x_{t-1} \mid z_{1: t-1}, u_{1: t}\right) \mathrm{d} x_{t-1}
$$

Using Markov's first assumption, equation 115 can be written as equation 116 .

$$
=p\left(z_{T} \mid x_{t}\right) \int_{x_{t-1}} p\left(x_{t} \mid x_{t-1}, u_{t}\right) p\left(x_{t-1} \mid z_{1: t-1}, u_{1: t}\right) \mathrm{d} x_{t-1}
$$

Using Markov's second assumption, equation 116 can be written as equation 117.

$$
=p\left(z_{T} \mid x_{t}\right) \int_{x_{t-1}} p\left(x_{t} \mid x_{t-1}, u_{t}\right) p\left(x_{t-1} \mid z_{1: t-1}, u_{1: t-1}\right) \mathrm{d} x_{t-1}
$$

As per the definition of $\operatorname{bel}\left(x_{t}\right)(111)$

$$
=p\left(z_{T} \mid x_{t}\right) \int_{x_{t-1}} p\left(x_{t} \mid x_{t-1}, u_{t}\right) \operatorname{bel}\left(x_{t-1}\right) \mathrm{d} x_{t-1}
$$

The equation 118 consist of two steps:

- Prediction step

$$
\overline{\operatorname{bel}}\left(x_{t}\right) \int_{x_{t-1}} p\left(x_{t} \mid x_{t-1}, u_{t}\right) \operatorname{bel}\left(x_{t-1}\right) \mathrm{d} x_{t-1}
$$

- Correction step

$$
\operatorname{bel}\left(x_{t}\right)=p\left(z_{T} \mid x_{t}\right) \overline{b e l}\left(x_{t}\right)
$$

Stochastic estimation techniques aim to estimate some kind of central tendency of the probability distribution of the variable (state) that is being estimated along with some other factors that are required to define the distribution. For example, the Kalman filter, which is a stochastic estimator, estimates the mean and the variance of the distribution.

Many of the stochastic estimation methodologies are aimed at the estimation of the mean of a given distribution, but that is not the only choice available. There are other measures of central tendency such as mean, median, mode, root mean square, geometric mean, or harmonic mean. The central tendency that is required to be estimated depends on the type of problem. For example, if there are many outliers in the system data, then median is a better measure of the true estimate than the mean. Similarly, if the distribution of the data is multi-modal, then estimating the mode of the distribution is a better option than estimating the mean. Although, at the same time, the mode estimation of a distribution can be difficult both mathematically and computationally. The advantages and disadvantages of the mean, median and mode estimation are given below:

- Mean

- Minimizes the mean square error

- Mathematically easiest to implement

- Usually computationally efficient

- Highly sensitive to outliers

- Median

- Minimizes the mean absolute error

- Can be difficult to implement computationally and mathematically

- Less sensitive to outliers than mean 
- Mode

- Minimizes the the most probable error

- Well-suited for multi-modal distributions

- Might be highly inaccurate

For symmetric distributions with the highest mode at the point of symmetry, all the three estimates are the same.

The Bayesian filter is framework of recursive stochastic state estimation. It has multiple realisations. Among the most popular ones are the Kalman filter, the unscented Kalman filter and the particle filter. Different realizations have different properties, such as

- different distribution

- parametric or non-parametric filter

- linear or nonlinear models (observation and system models)

\subsection{Extended Kalman Filter}

The Kalman Filter is the most well-known and most widely used realisation of the Bayesian filter explained above. Algorithm 4 gives the linear Kalman filter algorithm. Kalmanfitering is used widely in the aerospace industry.

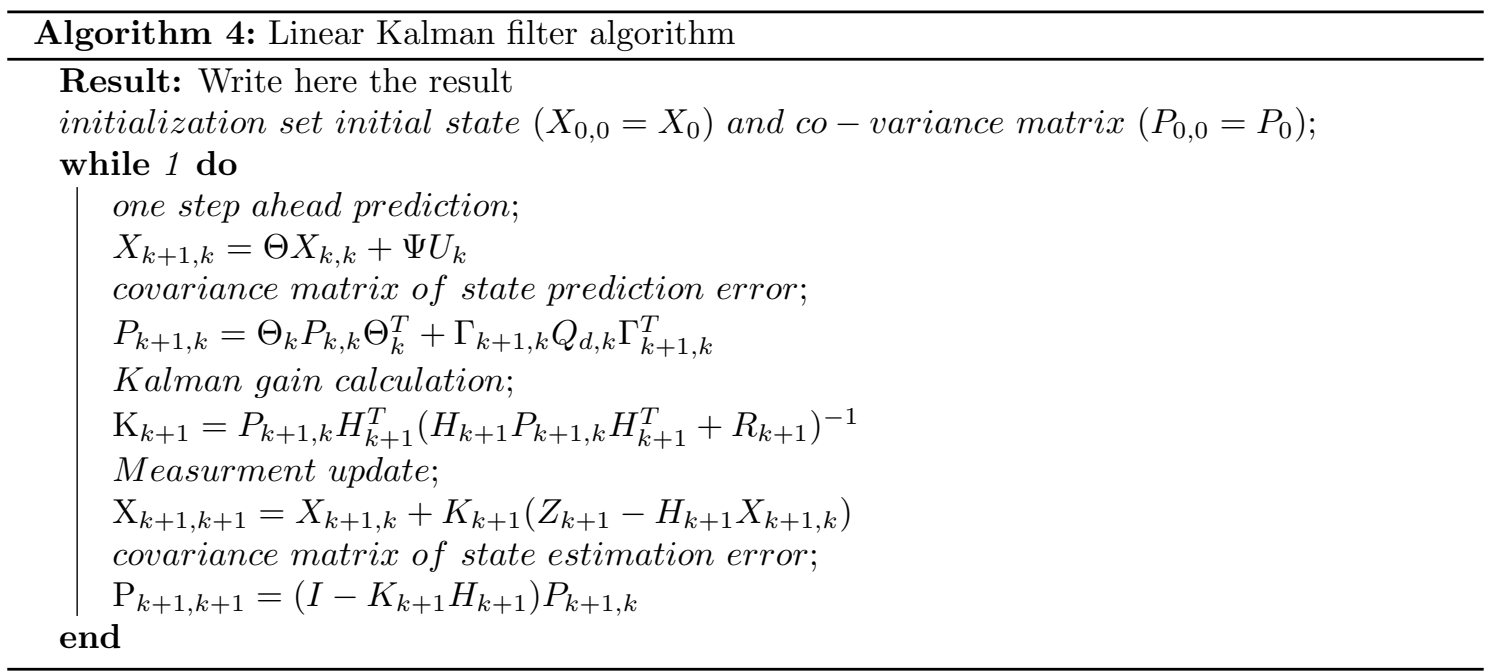

The linear discrete system is given by equation 119, where $X_{k, k}$ is the current optimal estimated state vector, $X_{k+1, k}$ is the one step ahead predicted state vector, $X_{k+1, k+1}$ is the one step ahead optimal state estimation, $w_{d, k}$ is the system noise, $U_{k}$ is the system input, $Z_{k+1}$, is the system output, $v_{k+1}$ is the sensor noise $\Theta$ is the system state matrix, $\Psi$ is the system input matrix, $\Gamma$ is the system noise matrix and $H_{k+1}$ is the sensor measurement matrix.

$$
\begin{gathered}
X_{k+1}=\Theta_{k+1, k} X_{k}+\Psi_{k+1, k} U_{k}+\Gamma_{k+1, k} w_{d, k} \\
Z_{k+1}=H_{k+1} X_{k+1}+v_{k+1}
\end{gathered}
$$

The Kalman filter is only applicable to linear Gaussian systems. To be precise, the Kalman filter is only applicable to continuous systems, but with a minor change it is also applicable to discrete systems (discrete Kalman filter). The following assumptions must hold for a Kalman filter to work.

- Both the Markov assumptions must hold.

- State transition probability must be linear in nature. This means that the system (process model) must be linear as well. 
- Measurement probability must be linear in nature. This implies that the measurement model must be linear as well.

- All the distributions (system noise, sensor noise, etc.) should be Gaussian distributions.

A Kalman filter estimate is the best optimum estimate, provided all the assumptions hold true. Figure 8.1 illustrates the estimation process of a Kalman filter.

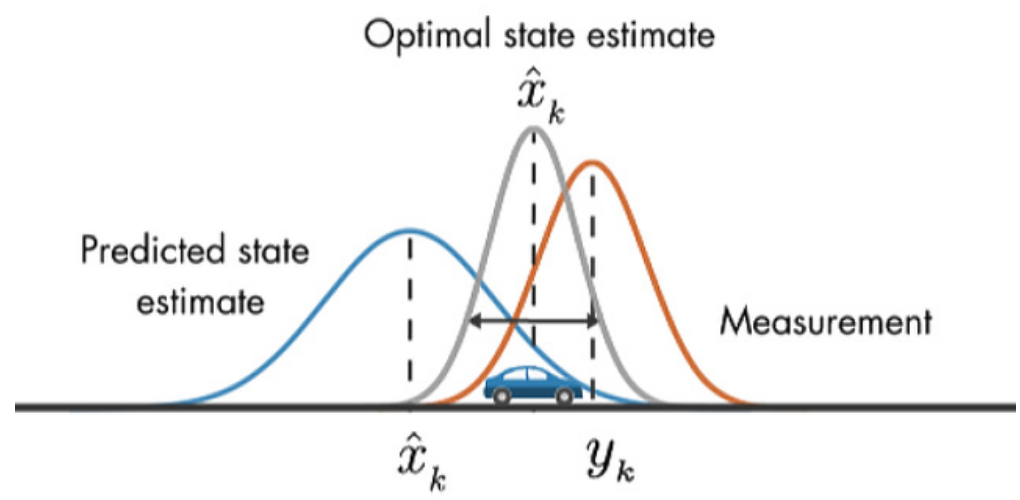

Figure 8.1: Kalman filter illustration

In reality, hardly any system is linear in nature and thus the traditional Kalman filter cannot be used for estimation. For nonlinear systems, the extended Kalman filter can be used for the state estimation, It is an extension of the original Kalman filter. Since the Kalman filter is only applicable to linear systems, a nonlinear system is linearized about the previous optimal states by using the Taylor series expansion (Jacobean matrix). For discrete cases, the obtained linear system is discretized further. The EKF algorithm is presented in algorithm 5, the equation 120 provides the system equation.

$$
\begin{gathered}
\dot{X}(t)=f(X(t), U(t), t)+G(X(t), t) \\
Z_{t}(t)=h(X(t), U(t), t)
\end{gathered}
$$

EKF utilises the first-order Taylor series expansion for linearising the nonlinear systems, but more accurate versions of EKF have been developed which use the second-order Taylor series expansion for the linearisation, also called EKF2 (Vathsal (1987) and Roth and Gustafsson (2011)). These methods have been shown to have a higher co-variance domain. Although $E K F 2$ is more accurate, it is not used a lot in practice due to its high computational cost, slow execution time and high complexity. It is not possible to prove the global convergence of any EKF variants. Thus, if the initial estimate has a large error, the algorithm may diverge or provide a poor quality of estimates, 
as shown by Crassidis et al. (2007a), Song and Grizzle (1995), and Psiaki (2005).

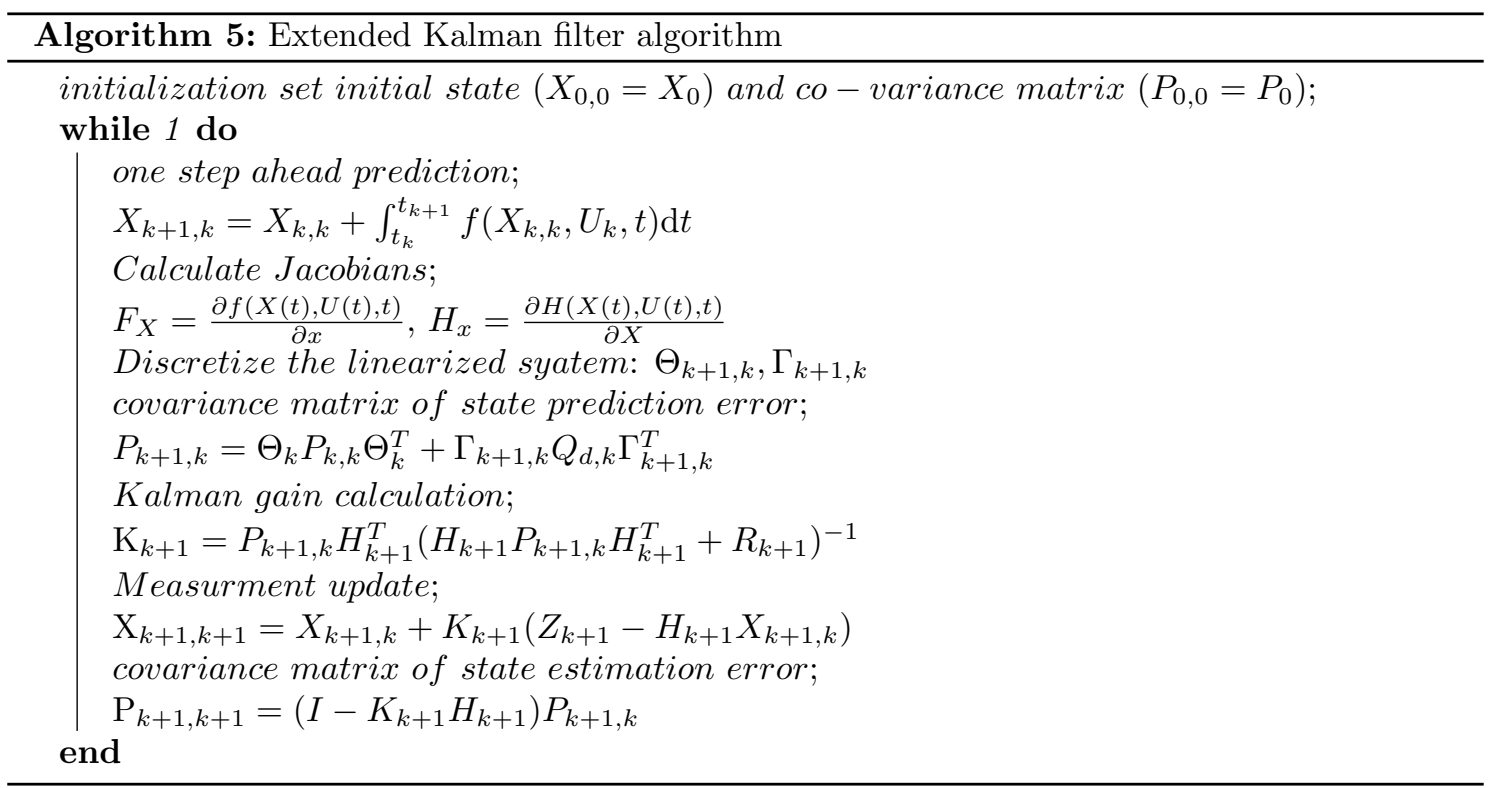

Majorly, two forms of EKF are used in the attitude estimation of aerospace vehicles: MEKF (Multiplicative Extended Kalman Filter) and AEKF (Additive Extended Kalman Filter). Both the methods are based on quaternion representation of the attitude. The major difference between the two schemes is that in MEKF, the attitude quaternion is considered as a rotation vector and the error between the quaternions is given by equation 19. However, under AEFK each member of the quaternion is treated as a separate independent parameter and the error is given by simple algebric subtraction. Bonnabel et al. (2009b) and Martin and Salaün discuss extensions of MEKF for GPS-aided attitude estimations systems.

As all forms of EKF require linearisation of the system, the estimate is plagued by linearisation errors. These errors are significant if the system is highly nonlinear. The Taylor series expansion is a poor approximation of the nonlinear function. Furthermore, for a highly nonlinear system, calculating the Jacobians is a very cumbersome task. There exist multiple variants of EKF, such as the iterative extended Kalman filter, the adaptive extended Kalman flter, the dual extended Kalman filter, etc., but for low-cost attitude estimation systems, mostly the simple EKF and MEKF are used, because other methods have higher computational costs.

\subsection{Unscented Kalman Filter}

The unscented Kalman Filter is another realisation of the Bayesian filter. It is a variant of the Kalman filter that is capable of state estimation of nonlinear systems similar to the EKF. The method has been advocated as an alternative to the popular EKF in various papers (e.g., Julier and Uhlmann (2004), Crassidis (2006), Crassidis et al. (2007b)). The method parametrizes the states in Eucledian states, and the mean and co-variance are recalculated after the transformation of the parameters through the nonlinear function. The underlying assumption is that calculation of mean and co-variance of a nonlinear function utilizing the transformed parameters is easier than linearizing the system. UKF uses sigma points, which are the specially selected points on the original distribution that are transformed through the nonlinear function (system model). Then the mean and the variance of the transformed sigma points is calculated to obtain a better Gaussian approximation of the real transformed distribution.

There are multiple forms of UKF, which mainly differ from each other in the way the sigma points are selected. The algorithm of UKF is given below 6 . Let the equation 121 represent the $\mathrm{n}$ state discrete time nonlinear system. Li (2014) provides a brief overview about the UKF algorithm. The algorithms, as well as the advantages and disadvantages of other forms of UKF such as the general unscented Kalman filter, the simplex unscented Kalman filter and the spherical unscented 
Kalman filter, can be found in detail in Simon (2006).

$$
\begin{gathered}
X_{k+1}=f\left(X_{k}, U_{k}, t_{k}\right)+w_{k} \\
Y_{k}=h\left(X_{k}, t_{k}\right)+v_{k} \\
w_{k} \sim\left(0, Q_{k}\right) \\
v_{k} \sim\left(0, R_{k}\right)
\end{gathered}
$$

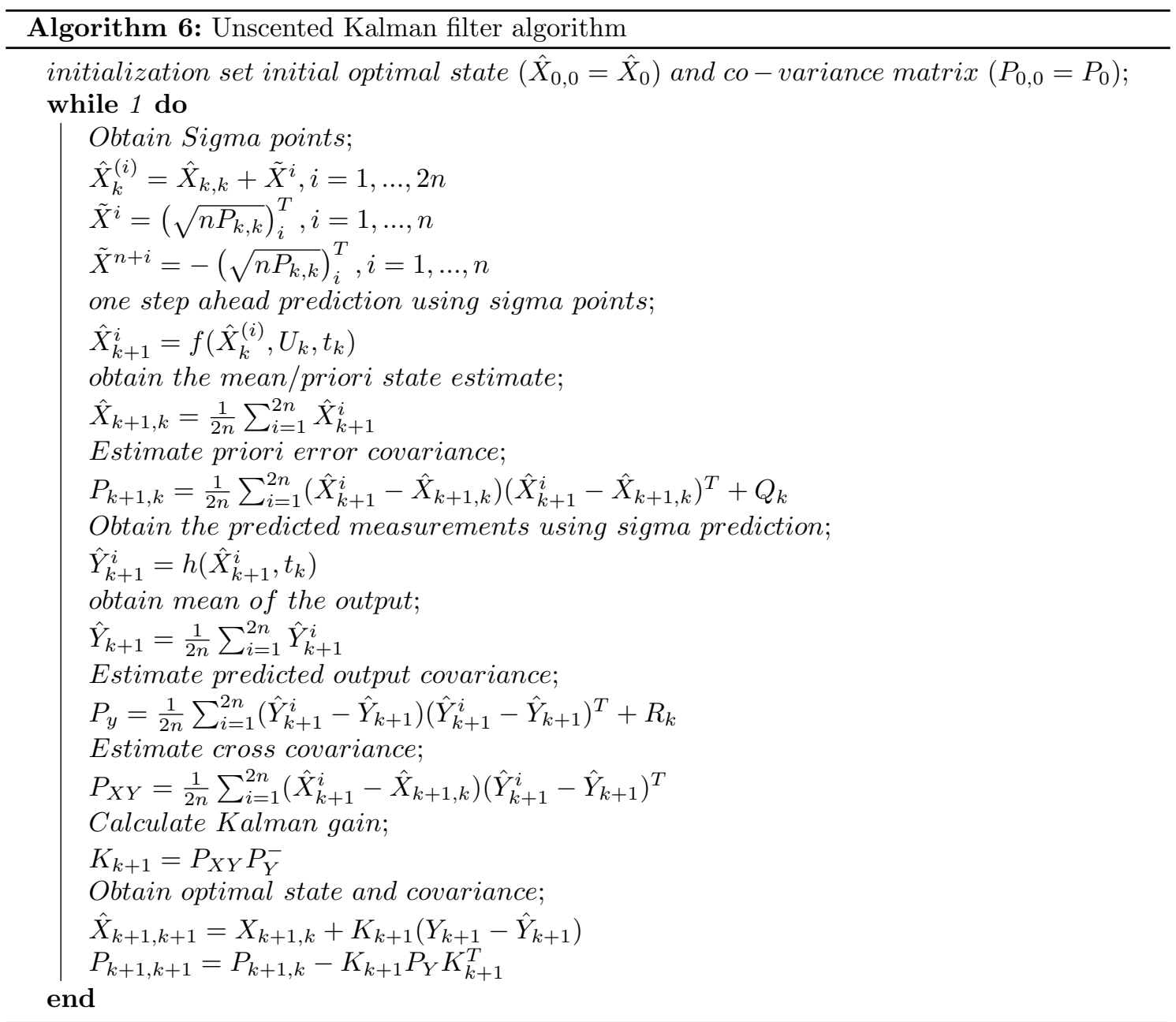

When the system is linear, the original distribution after transformation (system model) stays the same type of distribution. Thus, if the original state was described by a Gaussian distribution, the transformed distribution is also Gaussian. But in case of a nonlinear system, the shape and the properties of the original distribution change after the nonlinear system transforms. Thus, a Gaussian approximation of the transform is needed to get the mean and variance (co-variance) of the transformed distribution. In EKF, the transformation function (system model) is made linear by using a Taylor series expansion and in the case of UKF, multiple points (sigma points) are chosen, which are used after the transformation to obtain the Gaussian approximation of the transformed distribution. 


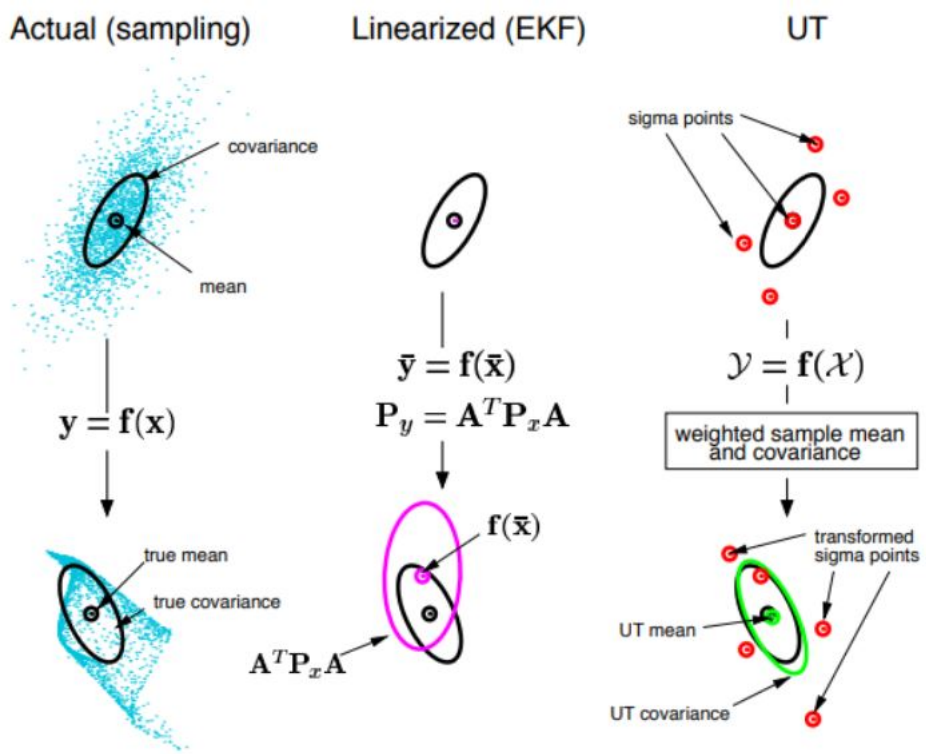

Figure 8.2: illustration of the difference between EKF and UKF

Figure 8.2 graphically illustrates the difference between EKF and UKF.

Särkkä (2007) derives a continuous-discrete time UKF and uses it to estimate the states of a nonlinear system whose process model (continuous time) and sensor model (discrete time) are available. Singer (2006) compares the continuous-discrete time UKF with a EKF. Banani and Masnadi-Shirazi (2007) introduces IUKF (Iterated UKF) and compares it to the existing EKF, IEKF, and UKF. According to the author's results, as the nonlinearity of the system increases, UKF and IUKF perform better than IEKF and EKF, while IUKF has a faster convergence than UKF. For a linear system or a system with a small nonlinearity, IEKF performs best, followed by IUKF, EKF and UKF in the respective order, although the absolute difference in error performance is negligible.

Khalaf et al. (2017) introduces a novel adaptive UKF. The filter is based on adaptive filtering and UKF. The filter is able to estimate the states of the system subjected to time-varying and unknown measurement noise. The filter utilises a real-time statistic noise tracker, based on the forgetting factor concept. The filter is implemented on a UAV and provides better results than a simple UKF. Furthermore, Wan et al. (2004) state that the UKF is more accurate and robust than the EKF, while having comparable computation costs.

Recently, another filter called 'Particle Filter' has been developed, based on Gordon et al. (1993). It is a generalised form of the UKF. In particle filtering, instead of using sigma points to recreate the probability density function, a desired number of random points are taken, which are used to obtain the probability density function after the nonlinear transformation. A method similar to particle filters is very computationally heavy (Crassidis et al. (2007b)), although it provides a very high accuracy of results. Cheng and Crassidis (2004), Liu et al. (2007) and Carmi and Oshman (2009) have developed particle-filter based attitude estimation techniques, but as stated above, they are computationally costly.

Furthermore, Bonnabel et al. (2009b) suggested a new filter called Invariant Extended Kalman Filter (IEKF). It is based on the natural symmetry in the system dynamics and uses the symmetry to design the filter. Unfortunately, the novel method is not suitable for low-cost attitude estimation because of high computational costs. 


\subsection{Attitude estimation}

The different types of Kalman filters explained above are widely used in vehicle attitude estimation, especially in the field of UAVs.

Marins et al. (2001) proposes two methods to use an EKF for state estimation of the system (in the particular case, a human walking) using a MARG sensor. The method is based on quaternions, but can be translated into other attitude representation such as a rotation matrix (Euler angle based). In the first method, a process model consists of a simple single order system driven by white noise (this is the model assumed for a human walking) and the states of the system are three angular rates and four quaternions. The sensor model consists of the readings from the accelerometer, magnetometer and rate gyro.

Formulating an EKF using such a setup leads to highly nonlinear output sensor equations (for accelerometer and magnetometer), which in turn leads to high linearisation errors and high computational costs.

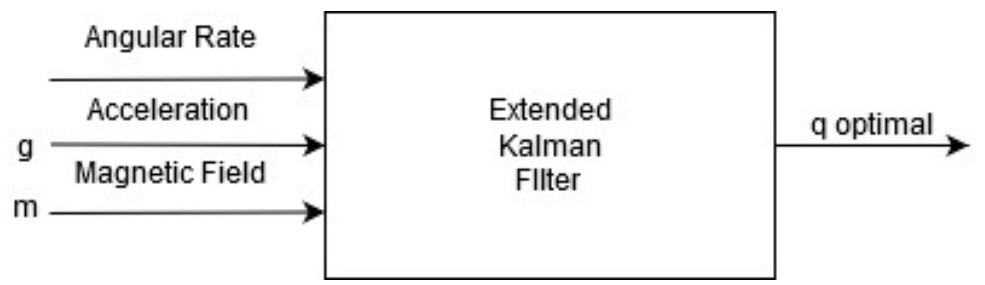

Figure 8.3: The schematic of EFK in Marins et al. (2001): Method 1

In the second proposed method, a Gauss-Newton method is used to compute the quaternion form of the accelerometer and magnetometer, which is then fed to a Kalman filter. It has the same process model as the first method, but the sensor model is linear at a direct angular rate and the quaternion is available to the Kalman filer. The second method is computationally more effective, but the success of the filter is completely dependent on the convergence of the Gauss-Newton method. The author argues that the Gauss-Newton method converges to a sufficient accuracy in a few iterations. Both the methods assume that the accelerometer reading is dominated by the gravity vector pointing downward in the inertial frame. Thus, if the system experiences a large and prolonged external acceleration, then these methods might fail.

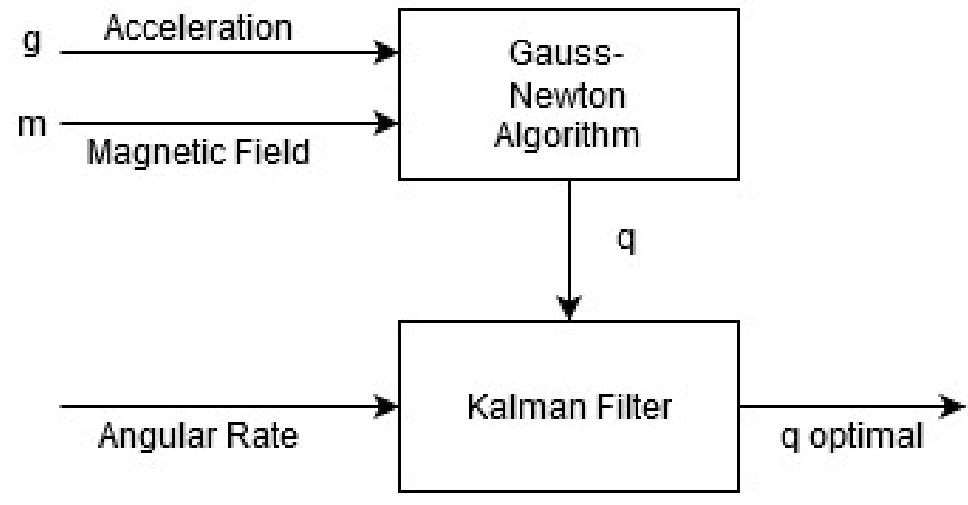

Figure 8.4: The schematic of Kalman filter in Marins et al. (2001): Method 2

The approach mentioned by Marins et al. (2001) is improved by Xiaoping et al. (2005) using a QUEST algorithm instead of the Gauss-Newton method. The benefit of QUEST over the GaussNewton algorithm is that it is a single frame/non-iterative algorithm, although $Q U E S T$ requires a $4 X 4$ matrix inverse. This approach also assumes that that the system has a zero external acceleration over an extended period of time. 


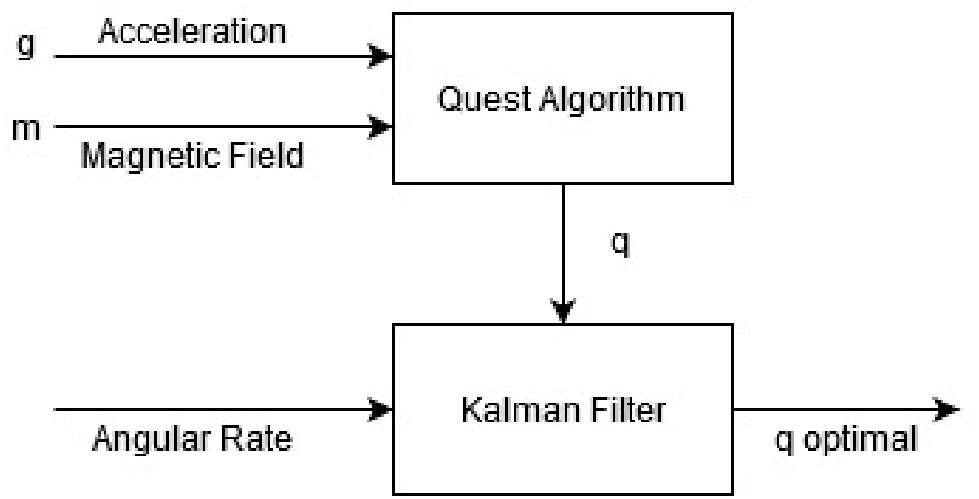

Figure 8.5: The schematics of the Kalman filter in Xiaoping et al. (2005)

Sabatini (2006) introduces another approach based on EKF. The main principal of the approach is to obtain the attitude quaternion via the rate gyroscope reading integration and then fuse them with the attitude quaternion obtained via accelerometer and magnetometer readings. The approach used by Sabatini is similar to that of the sensor fusion. The rate gyroscope readings are used in the process model and the accelerometer and magnetometer readings are a part of the sensor model. To reduce the impact of the external accelerations and magnetic disturbance, the sensor noise co-variance matrix $(R)$ is adapted based on the difference in the modulus of the sensor readings from the gravity and local earth's magnetic field.

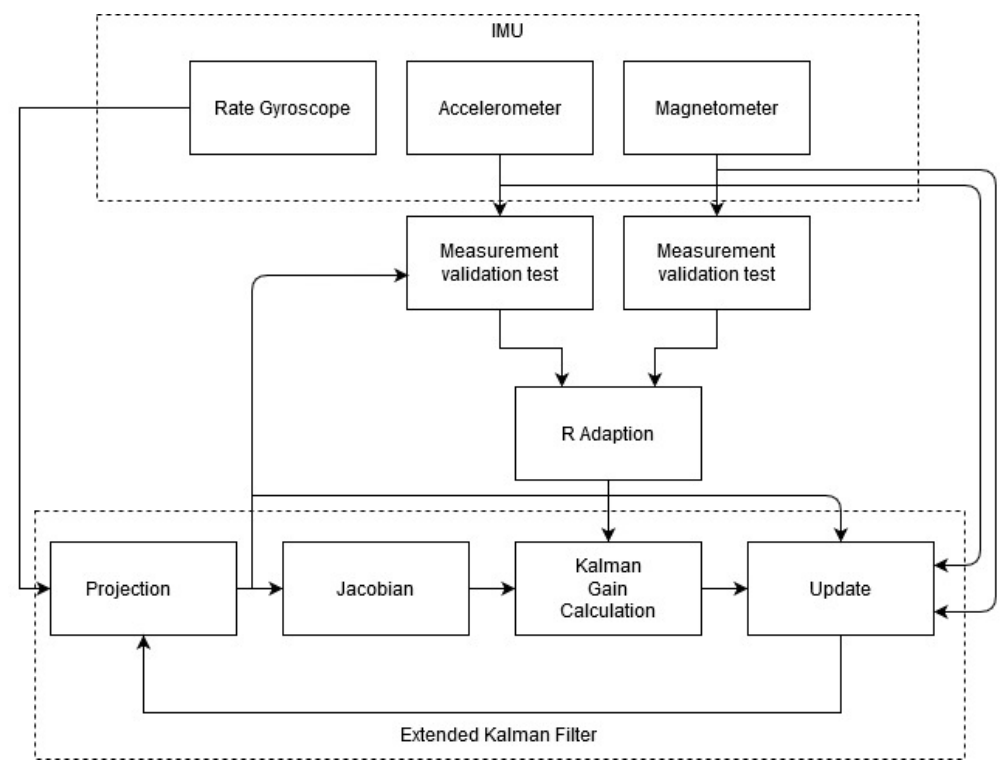

Figure 8.6: The schematic of EFK in the paper Sabatini (2006)

Zhang et al. (2012) designed a Kalman filter-based attitude estimation algorithm. The attitude is estimated using a sensor fission based on a linear Kalman filter. The rate gyroscope readings are used as a linear process model (gyro readings are integrated to obtain the attitude) and accelerometer and magnetometer readings are manipulated to create pseudo-readings. Furthermore, in order to check for external acceleration and magnetic disturbance, the modulus of accelerometer and magnetometer readings are checked against gravity and earth's magnetic field vector, and based on the error, the readings are manipulated. Although the algorithm shows satisfactory experimental results in cases of no or temporary external disturbance (acceleration or magnetic), the filter fails in case long-term interference exist.

Feng et al. (2017) use a two-step geometrically-intuitive correction algorithm to obtain the at- 
titude quaternion from the accelerometer and magnetometer by vector-matching of the readings obtained from the accelerometer and magnetometer (resolved in body reference frame) to the earth's gravity and magnetic field respectively. The obtained attitude quaternions are then fed to the Kalman filter as sensor readings, while the process model of the Kalman filter consist of the quaternions obtained via gyro readings (via numerical integration of the gyro readings). The main contribution of the paper to the field is the two-step geometrically-intuitive correction algorithm (TGIC). The TGIC method is explained in section 7.4. The major drawback of the technique is that the author assumes that the net external acceleration on the body over a period of time will tend to zero. This may not be always true in the case of the a failure of the state quadcopter.

Widodo and Wada (2016) use an external acceleration compensation scheme to remove the effects of the external acceleration from the attitude estimation. The author's approach is based on EKF and uses the acceleration compensation scheme to overcome the problem of external acceleration. The approach uses the system model to extract the external acceleration from the readings and uses it to adjust the measurement noise co-variance matrix. The method provides an insight that the system model can be used to compensate for the external acceleration, but the model and methods chosen by the author do not provide satisfactory results. Furthermore, since the author uses EKF, the computational cost compared to many aforementioned methods is higher.

De Marina et al. (2012) use a combination of the UKF and TRIAD algorithm to estimate the attitude of a UAV. The filter uses the TRIAD algorithm to obtain the DCM (direction cosine matrix), given the reference gravity and local magnetic field vector and the measured accelerometer and magnetometer readings. For the UKF, gyroscope readings are used for the process model and the DCM obtained earlier is used as the observation model. The designed filter is tested on a UAV and it shows to be able to deal with the nonlinearity of the system. However, when the filter utilises the TRIAD algorithm to obtain the observed attitude (the attitude given by observation/sensor model), the observed attitude is plagued by the shortcoming of the TRIAD algorithm (as explained in section 6.1). Figure 8.7 shows the schematics of the filter.

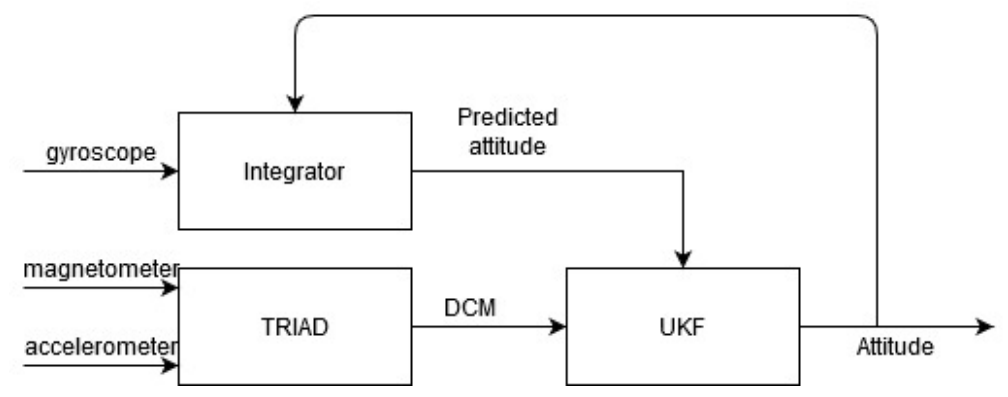

Figure 8.7: The schematic filter presented by De Marina et al. (2012)

Similarly, another technique utilising UKF for the purpose of attitude estimation is presented by Yuan et al. (2015). The estimator uses gyro readings as process model and the predicted attitude is obtained by simply integrating the gyro readings. The sensor model consist of nonlinear equation of accelerometer and magnetometer relations. The sensor noise co-variance matrix is adaptive in nature. The adaption is done using the innovation and co-variance matching approach (given by Mehra (1972)). The filter gives very satisfactory results according to the author. The major drawback of the filter is that the author assumes that there is not prolonged acceleration of the system. The schematics of the the filter are given in figure 8.8. 


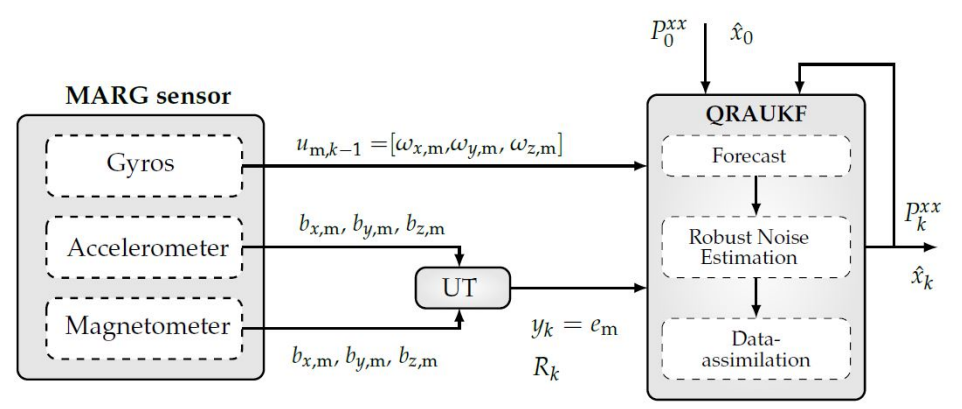

Figure 8.8: The schematic filter presented in the paper Yuan et al. (2015)

\subsection{Discussion}

This chapter surveyed the existing stochastic estimators. The most prominent filter is the Kalman filter. Since Kalman filtering is only applicable to linear systems, multiple extensions of the Kalman filter exist that can deal with non-linear systems (IEKF, EKF, UKF). The Kalman filter and its extensions are widely used in the aerospace industry for the purpose of attitude estimation across the field, including for quadcopters. These filters provide very satisfactory results for the task of attitude estimation, given that the quadcopter is in normal flight mode. However, the attitude estimate obtained by these filters worsens as the quadcopter accelerates and there is local magnetic field distortion. Some filters such as Yuan et al. (2015), Sabatini (2006), Feng et al. (2017), Widodo and Wada (2016), and Sabatini (2011) provide different schemes to cater for the problems of external acceleration and local magnetic field disturbance, but none of them provides satisfactory results if the disturbance lasts for a long period of time. Furthermore, as the number of estimated states increases, so does the computational cost, which is the biggest drawback of the Kalman based filters. Thus, it is not advisable to directly apply the filters without any modifications. But with the right modifications, the explained issues can be resolved, as I will discuss in the following chapter. 


\section{Conclusions}

The aim of this research is to develop an attitude estimation method for the outdoor environment that can estimate the states of a quadcopter with a single rotor failure with sufficient accuracy using only the on-board sensors. Thus, this preliminary report reviewed the available methods for the stated problem.

As was observed in chapter 6, all the deterministic approach methods such as FOAM, SVD, QUEST, and others are able to estimate the attitude of the system with sufficient accuracy only if the sensors are of good enough quality, i.e., if the sensor noise is comparatively low and the sensor readings are not corrupted due to external factors. However, in the case of low-cost quadcopters, low-cost sensors are used, which have a high sensor noise. Furthermore, the sensors used in the quadcopter are accelerometer and magnetometer. As explained in chapter 2, the accelerometer measures the specific force, but all the deterministic approach methods assume that accelerometer measurements are dominated by the gravity vector. It follows that the deterministic approach methods aim at obtaining a rotation matrix or a quaternion that rotates the accelerometer measurements such that they coincide with the gravity vector. These methods will work if the accelerometer measurements consist only of the gravity vector, but this assumption does not hold true in case of a quadcopter with a failed rotor, due to the fact that a quadcopter with a failed rotor spins rapidly about an axis and this leads to the accelerometer measurements being effected by the centrifugal force. In a system like a quadcopter, the accelerometer measurements consist of a superposition of gravity vector and the acceleration of the quadcopter.

A similar case is observed for the magnetometer: If there is no local magnetic field disturbance, the deterministic approach methods are a valid choice, even in the case of a quadcopter, as the motion of the quadcopter does not effect the reference local magnetic field. But in reality, it is hardly possible to ensure that there is no magnetic disturbance. The deterministic approach methods works well for a system like a satellite, as it utilises star sensors or star trackers, which track the stars (which, in this case, are assumed to be stationary or have an unknown motion) and thus a constant reference is always available that is not effected by external unknown disturbance. Since in the case of a quadcopter it is not possible to make sure that the accelerometer and magnetometer measurements are not effected by unknown quadcopter acceleration and unknown local magnetic field disturbance, the deterministic approach methods on their own are not able to estimate the attitude of the quadcopter with sufficient accuracy.

In chapter 7, complementary filters were discussed. Complementary filter-based attitude estimation techniques are among the most widely used attitude estimation techniques for a quadcopter. These algorithms are designed for low-cost systems, thus the computational cost of these algorithms is very low. All the filter designs made a few similar assumptions: Firstly, it is assumed that the accelerometer measurements are highly dominated by the gravity vector. This assumption holds true in the normal working of a quadcopter, as a quadcopter is not accelerating all the time and even then the acceleration is usually very low compared to the acceleration due to gravity. But in the case of a failed rotor, this assumption cannot be validated. Secondly, the majority of the filters assume that after a certain period of heating up, the gyro bias changes very slowly and thus no correction is implemented for the gyro bias. This problem is addressed in a few complementary filters (e.g., Madgwick et al. (2011), Mahony et al. (2008), Tian et al. (2013), Al-Fahoum and Abadir (2018)). Furthermore, the problem of local magnetic field disturbance is addressed using different schemes in different methods and all the methods have certain drawbacks associated with it. The complementary filter-based attitude estimation methods can be used for the stated research problem, but they need some modifications. The most promising candidate is the filter developed by Madgwick et al. (2011), due to its fast execution time and very accurate estimates.

In chapter 8 , stochastic attitude estimation methods are surveyed. The Kalman filter and its extentions (EKF, IEKF, UKF) are the most widely used stochastic filter and thus the back bone of the state estimation in the majority of systems. Although the Kalman filter and its extensions are computationally more expensive than the previous two branches of methods, the Kalman filter 
and its extinctions can accommodate both the system dynamics and the noise characteristic (of model and sensor) for state estimation. This gives the stochastic filter an edge over the other methods. Multiple algorithms have been developed using some form of Kalman filter for the purpose of the attitude estimation of different vehicles. For a system like the quadcopter, Kalman filters have proved to provide a highly satisfactory attitude estimation. The major problem, however, that is observed in most of the Kalman filter-based methods are the assumptions made while developing the filter. Most authors made the assumption that accelerometer measurements are highly dominated by the gravity vector. As mentioned previously, this assumption does not hold true in case of a quadcopter with a failed rotor. Multiple algorithms utilise different schemes to address this problem, but in the case of a prolonged quadcopter acceleration, they all might fail. There is a similar case for the magnetic field distortion. While this can be addressed through modifications, the biggest drawback of the Kalman filter is still the computational cost.

Thus, on its own any of the discussed attitude estimation algorithms cannot address the problem of a quadcopter with a failed rotor. However, a combination of them can be used in conjugation with a quadcopter system model that can be used to remove the external accelerations from the accelerometer so that the readings are dominated by the gravity vector. Although the EKF and UKF provide the most accurate estimation, their computational costs are higher than the other two methods. However, their costs can be decreased by decreasing the number of states of the filter, and gyro biases can be corrected using complementary filtering as done by Madgwick et al. (2011). To remove the magnetic field distortions, different methods have been explored by various authors, which combined with the other suggestions should render effective.

\subsection{Preliminary Filter Design}

It was observed that on its own, none of the already existing methods can be used to address the problem of quadcopter with a failed rotor, but a combination can be effective. A Preliminary Filter Design is introduced here that can estimate the attitude of a quadcopter with a failed rotor. A rough schematics of the filter is shown in the figure 9.1.

The filter consists of five parts. Part one takes the accelerometer readings and the quadcopter model's (simple model) predicted readings and subtracts them in order to obtain the gravity vector in the body frame of reference. Then the obtained vector is checked by utilising the modulus operation and the noise variance is adjusted accordingly (either using an adaptive technique or a parabolic equation). In the second part of the filter, the obtained obtained vector is used to predict the attitude quaternion using a gradient descent algorithm.

Since only the corrected accelerometer reading is used to obtain the attitude, the yaw estimate is not reliable (it will be corrected in the later steps). In the third part of the filter, a simple linear Kalman filter is used to obtain the optimised attitude estimate using the rate gyro readings and the predicted attitude quaternion. In the fourth part, the gyro bias is corrected using the integral gain correction introduced in the Mahony filter. In the fifth part, the yaw estimate obtained using the magnetometer reading is fused with the yaw estimate obtained via the gyroscope yaw rate reading by using a linear complimentary filter. The gain of the complimentary filter is obtained using fuzzy logic. The magnetometer readings are compensated using the ellipse hypothesis compensation, and then the compensated readings are used to obtain the yaw estimate. 


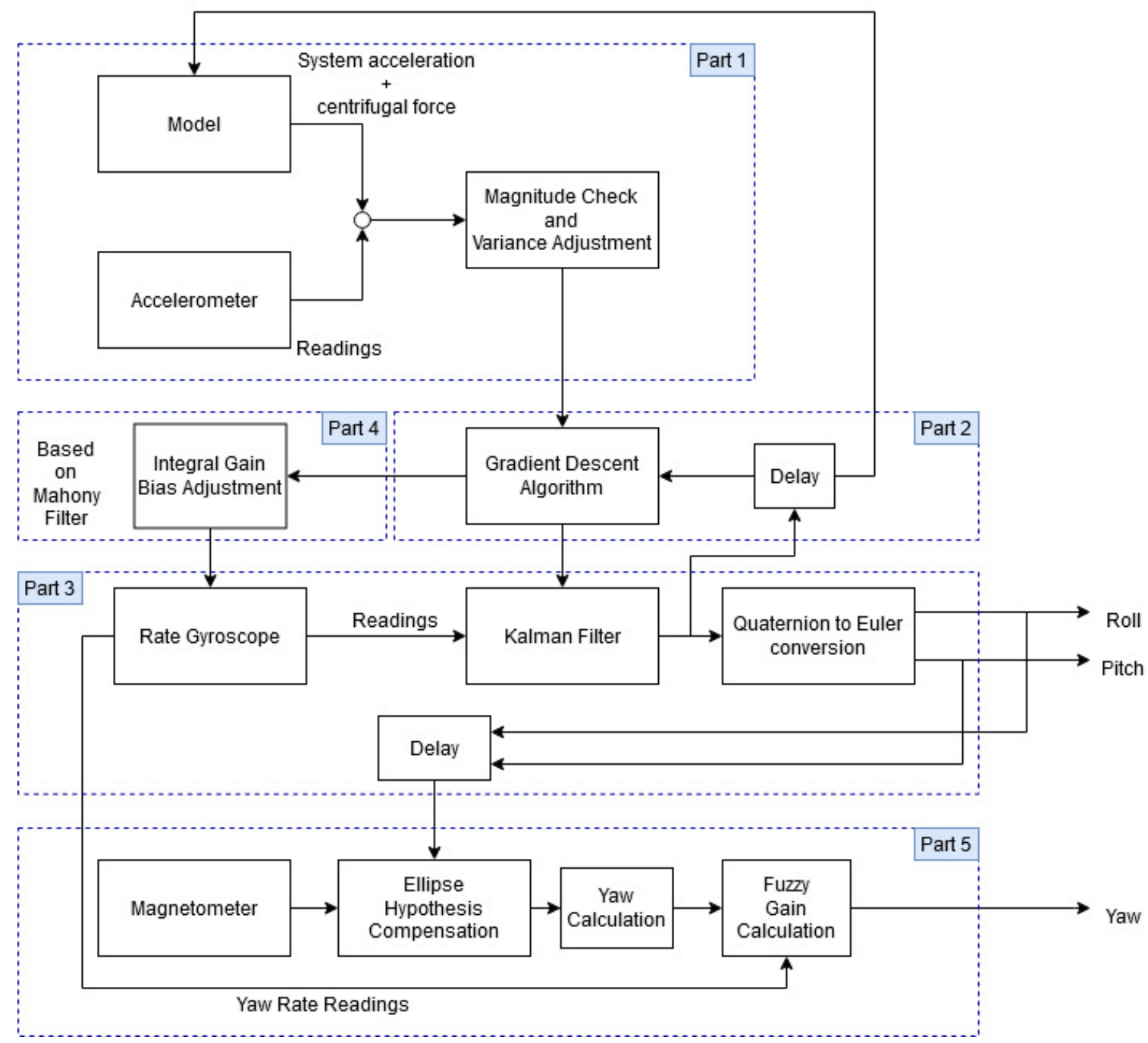

Figure 9.1: Schematics of current proposed filter

Part one of the filter utilises a reduced quadcopter model to obtain the linear accelerations of the quadcopter. The reduced model is given in the equation 122

$$
m \vec{a}_{B}=m \vec{g}_{B}+\vec{F}_{T_{B}}
$$

where

$$
\begin{gathered}
m \vec{g}_{B}=m|g|\left[\begin{array}{lll}
0 & 0 & g
\end{array}\right] R_{B / I} \hat{E}_{I} \\
{\left[\hat{E}_{I}\right]=\left[\begin{array}{lll}
\hat{x}_{i} & \hat{y}_{i} & \hat{z}_{i}
\end{array}\right]^{T}} \\
\vec{F}_{T_{B}}=K\left[\begin{array}{lll}
0 & 0 & \sum_{i=1}^{4} \omega_{i_{\text {rot }}}^{2}
\end{array}\right]^{T} \hat{E}_{B}
\end{gathered}
$$

where $\hat{x}_{i}, \hat{y}_{i}, \hat{z}_{i}$ are the unit vectors along the $x, y, z$ axis of the inertial frame respectively. Furthermore, the centrifugal force measured by the accelerometer is given by equation 123:

$$
\vec{a}_{\omega}=\vec{\omega}_{B} \times\left(\vec{\omega}_{B} \times \vec{r}_{B}\right)
$$

where $\vec{\omega}_{B} \times$ is the rate gyro reading of the current time step and $\vec{r}_{B}$ is the displacement that is to be identified beforehand. Thus, the total external acceleration $\vec{a}_{T}$ is given by equation 124

$$
: \vec{a}_{T_{B}}=\vec{a}_{\omega}+\vec{a}_{B}
$$


The obtained acceleration is subtracted from the accelerometer reading of the current time step.

$$
\vec{a}_{\text {corr }}=\vec{a}_{a c c}-\vec{a}_{T_{B}}
$$

In part two of the filter, the obtained $\vec{a}_{c o r r}$ is fed to the gradient descent algorithm to obtain the $q_{\text {meas }}$.

$$
\begin{aligned}
& q_{\text {meas }}=q_{t-1}-\beta \dot{q}_{\text {meas }} \\
& \dot{q}_{\text {meas }}=\frac{\Delta f}{\|\Delta f\|} \\
& \Delta f=J_{g}^{T}\left(\hat{q}_{t-1}\right) f_{g}\left(\hat{q}_{t-1}, \hat{a}_{t}\right) \\
& f_{g}(\hat{q}, \hat{a})=\left[\begin{array}{l}
2\left(q_{2} q_{4}-q_{1} q_{3}\right)-a_{\operatorname{corr}_{x}} \\
2\left(q_{1} q_{2}+q_{3} q_{4}\right)-a_{\text {corr }_{y}} \\
2\left(\frac{1}{2}-q_{2}^{2}-q_{3}^{2}\right)-a_{\text {corr }_{z}}
\end{array}\right] \\
& J_{g}(\hat{q})=\left[\begin{array}{cccc}
-2 q_{3} & 2 q_{4} & -2 q_{1} & 2 q_{2} \\
2 q_{2} & 2 q_{1} & 2 q_{4} & 2 q_{3} \\
0 & -4 q_{2} & -4 q_{3} & 0
\end{array}\right]
\end{aligned}
$$

where $q_{t-1}$ is the optimum attitude quaternion from the last time step.

In part three of the filter, the obtained $q_{\text {meas }}$ is fed to a linear Kalman filter as the sensor model. The process model is given by the rate gyro readings.

$$
\begin{gathered}
\dot{q}_{\text {predicted }}=\frac{1}{2} q_{t-1} \otimes\left[\begin{array}{ll}
0 & \omega_{t}
\end{array}\right]^{T} \\
q_{\text {predicted }}=q_{t-1}+\dot{q}_{\text {predicted }} \Delta t \\
P_{k+1, K}=\Phi P_{k, k} \Phi^{T}+\Lambda Q \Lambda^{T} \\
K=P_{k+1, K} H^{T}\left(H P_{k+1, K} H^{T}+R\right)^{-1} \\
q_{t}=q_{\text {predicted }}+K\left(q_{\text {meas }}-H q_{\text {predicted }}\right) \\
P_{k+1, k+1}=\left(I_{4 \times 4}-K H\right) P_{k+1, k}
\end{gathered}
$$

where $\omega_{t}$ is the current gyroscope reading, $Q$ is the process noise matrix which is given by the equation 128 , and $R$ is the sensor noise.

$$
\frac{\Delta t}{4} q \otimes\left[\begin{array}{rrrr}
0 & 0 & 0 & 0 \\
0 & \sigma_{\omega_{x}}^{2} & 0 & 0 \\
0 & 0 & \sigma_{\omega_{y}}^{2} & 0 \\
0 & 0 & 0 & \sigma_{\omega_{z}}^{2}
\end{array}\right] \otimes q^{T}
$$

The matrix $H$ is just an identity matrix of size $4 \times 4$, the matrices $\Phi$ and $\Lambda$ are discrete versions of a zero matrix and identity matrix of size $4 \times 4$ respectively, and $\sigma_{\omega_{x}}^{2}, \sigma_{\omega_{y}}^{2}, \sigma_{\omega_{z}}^{2}$ are the rate gyro noise characteristics.

The $\mathrm{R}$ matrix can be adjusted in an additive manner or be set equal to equation 129 :

$$
R=J^{T}\left[\sigma_{a}^{2}\right]\left[\sigma_{a}^{2}\right]^{T} J
$$

where:

$$
J=J_{g}(q)
$$




$$
\sigma_{a}^{2}=\left[\begin{array}{ccc}
\sigma_{a_{x}}^{2} & 0 & 0 \\
0 & \sigma_{a_{y}}^{2} & 0 \\
0 & 0 & \sigma_{a_{z}}^{2}
\end{array}\right]
$$

$\sigma_{a}^{2}$ is the noise characteristics of the accelerometer and $\sigma_{m}^{2}$ is the noise characteristics of the magnetometer.

In the fourth part, the rate gyro bias drift is compensated using an integral feedback of the error in the rate of change of attitude. This is shown in equation 130:

$$
\begin{aligned}
\omega_{t} & =\omega_{t}-\omega_{\text {corr }} \\
\omega_{\text {corr }} & =\zeta \sum_{t} \omega_{\text {err }} \Delta t \\
\omega_{\text {err }} & =2 q_{t-1} \dot{q}_{\text {meas }}
\end{aligned}
$$

The filter gain $\beta$ are set such that beta is equal to the modulus of the current rotation rate of the quadcopter multiplied by the time step. $\zeta$ is $\sqrt{\frac{3}{4}} \dot{\omega}_{d r i f t}$, where $\dot{\omega}_{\text {drift }}$ is the estimated rate of the gyro bias drift.

Finally, in the fifth part, the yaw estimate is obtained using equation 131:

$$
\psi=\varepsilon \psi_{m a g}+(1-\varepsilon) \psi_{\text {gyro }}
$$

where:

$$
\begin{gathered}
\psi_{\text {mag }}=\arctan \left(\frac{m_{y_{c o m}}}{m_{x_{c o m}}}\right) \\
\psi_{\text {gyro }}=\psi_{t-1}+\omega_{z} \Delta t \\
{\left[\begin{array}{l}
m_{x_{c o m}} \\
m_{y_{c o m}} \\
m_{z_{c o m}}
\end{array}\right]=\left[\begin{array}{c}
m_{x} \cos (\phi)-m_{z} \sin (\phi) \\
m_{x} \sin (\theta) \sin (\phi)+m_{y} \cos (\theta)+m_{z} \sin (\theta) \cos (\phi) \\
m_{x} \cos (\theta) \sin (\phi)+m_{y} \sin (\theta)+m_{z} \cos (\theta) \cos (\phi)
\end{array}\right]}
\end{gathered}
$$

The gain $\varepsilon$ is calculated using fuzzy logic to safeguard against magnetic field distortion and gyro saturation. Furthermore, since only the yaw estimation utilises the magnetometer reading, the magnetic distortion does not effect the roll and pitch estimates.

\subsection{Research Plan Summary}

- Design an attitude estimator as outlined in the previous section.

- Validate the estimator using an already existing simulation.

- Conduct flights with the Parrot Bepop 2 drone to obtain the real time flight test data.

- The raw sensor data will be fed to the attitude estimator to obtain the attitude of the system, which will be validated against the attitude estimates gained through the OptiTrack system.

- If successful, it will show that the filter is working as well as the OptiTrack system.

To achieve the main objective of the research, firstly a thorough review on the sources of error and shortcomings of the on-board sensors needed to be performed, which has been done in this literature review. The literature review also served to identify the most popular attitude estimation methods in the field of online quadcopter attitude estimation as well as their shortcomings that prevent them from being suitable for estimating the states of a quadcopter in a high-speed yaw rate spinning condition while only using the listed on-board sensors. 
After this, an attitude estimator needs to be developed, taking into account existing techniques that can cater for all the sensor errors and is able to estimate the attitude of the quadcopter with sufficient accuracy. The state estimator is being designed using Matlab.

The next task is to use an existing high-fidelity simulation developed by the aerospace control group (Sun et al. (2018a)) to validate the developed attitude estimator. The high-fidelity model on which the simulation is based on is obtained via the grey-box modeling technique. The model is verified against the real parrot bebop drone for wind velocity up to $10 \mathrm{~m} / \mathrm{sec}$. The quadcopter simulation used to validate the estimator has also been developed on Simulink and Matlab. The simulation is based on the high-fidelity model of a quadcopter developed by Sun et al. (2018a). The model is based on the Parrot Bebop 2 drone, which is why all the experiments will be carried out on the same drone.

In the next step, the real-time sensor data and true estimates from the OptiTrack system have to be collected by conducting flight tests on the Parrot Bepop 2 drone in the CyberZoo of TU Delft. The CyberZoo is fitted with an OptiTrack system and thus best suited to obtain the true attitude estimates. The quadcopter sensor readings are stored within the quadcopter and can be easily extracted after each flight test. The attitude estimation provided by the OptiTrack system comes as close as possible to the real attitude estimate of the quadcopter. Although the attitude estimates by the OptiTrack system might have some errors, they are very small and are considered to be zero and thus the OptiTrack system obtained attitude estimates are used as true estimates for validating the developed estimator.

The collected sensor data must be fed to the developed estimator in order for it to be validated against the collected true estimates in an open loop setup. If the estimator is able to estimate the quadcopter attitude with a sufficient accuracy, then the filter is working and the research objective is has been reached. 


\section{References}

A. S. Al-Fahoum and M. S. Abadir. Design of a Modified Madgwick Filter for Quaternion-Based Orientation Estimation Using AHRS. International Journal of Computer and Electrical Engineering, 10(3):174-186, 2018. 10.17706/ijcee.2018.10.3.174-186.

A. J. Baerveldt and R. Klang. Low-cost and low-weight attitude estimation system for an autonomous helicopter. IEEE International Conference on Intelligent Engineering Systems, Proceedings, INES, pages 391-395, 1997. 10.1109/ines.1997.632450.

S. A. Banani and M. A. Masnadi-Shirazi. A new version of unscented Kalman filter. World Academy of Science, Engineering and Technology, 26(2):192-197, 2007.

I. Y. Bar-Itzhack. Request: a recursive quest algorithm for sequential attitude determination. Journal of Guidance, Control, and Dynamics, 19(5):1034-1038, 1996.

Z. Benić, P. Piljek, and D. Kotarski. Mathematical modelling of unmanned aerial vehicles with four rotors. Interdisciplinary Description of Complex Systems, 14:88-100, 01 2016. 10.7906/ indecs.14.1.9.

B. Boada, M. Boada, and V. Diaz. Vehicle sideslip angle measurement based on sensor data fusion using an integrated anfis and an unscented kalman filter algorithm. Mechanical Systems and Signal Processing, 72-73:832-845, 2016a. 10.1016/j.ymssp.2015.11.003.

B. L. Boada, M. J. L. Boada, A. Gauchía, E. Olmeda, and V. Díaz. Sideslip angle estimator based on ANFIS for vehicle handling and stability. Journal of Mechanical Science and Technology, 29 (4):1473-1481, 2015a. ISSN 1738494X. 10.1007/s12206-015-0320-x.

B. L. Boada, M. J. L. Boada, A. Gauchía, E. Olmeda, and V. Díaz. Sideslip angle estimator based on anfis for vehicle handling and stability. Journal of Mechanical Science and Technology, 29 (4):1473-1481, 2015b. 10.1007/s12206-015-0320-x.

B. L. Boada, M. J. Boada, and V. Diaz. Vehicle sideslip angle measurement based on sensor data fusion using an integrated ANFIS and an Unscented Kalman Filter algorithm. Mechanical Systems and Signal Processing, 72-73:832-845, 2016b. ISSN 10961216. 10.1016/j.ymssp.2015.11 .003. URL http://dx.doi.org/10.1016/j.ymssp.2015.11.003.

S. Bonnabel, P. Martin, and E. Salaun. Invariant extended Kalman filter: Theory and application to a velocity-aided attitude estimation problem, page 297-1304. 2009b.

J. Calusdian, X. Yun, and E. Bachmann. Adaptive-gain complementary filter of inertial and magnetic data for orientation estimation. Proceedings - IEEE International Conference on Robotics and Automation, pages 1916-1922, 2011. ISSN 10504729. 10.1109/ICRA.2011.5979957.

A. Carmi and Y. Oshman. Fast particle filtering for attitude and angular-rate estimation from vector observations. Journal of Guidance, Control, and Dynamics, 32(1):70-78, 2009. 10.2514/ 1.36979 .

A. Cavallo, A. Cirillo, P. Cirillo, G. De Maria, P. Falco, C. Natale, and S. Pirozzi. Experimental comparison of sensor fusion algorithms for attitude estimation. IFAC Proceedings Volumes (IFAC-PapersOnline), 19:7585-7591, 2014. ISSN 14746670. 10.3182/20140824-6-za-1003.01173.

Y. Chang, T. Jiang, and Z. Pu. Adaptive control of hypersonic vehicles based on characteristic models with fuzzy neural network estimators. Aerospace Science and Technology, 68:475-485, 2017. 10.1016/j.ast.2017.05.043.

Y. Cheng and J. L. Crassidis. Particle filtering for sequential spacecraft attitude estimation. 2004.

Y. Cheng and M. D. Shuster. The speed of attitude estimation. Advances in the Astronautical Sciences, 127:101-116, 2007a. 
Y. Cheng and M. D. Shuster. simple kalman filter and smoother for spacecraft attitude. Advances in the Astronautical Sciences, 37(1):89-106, 2007b.

D. Choukroun. Adaptive optimal-request algorithm for attitude determination. AIAA Guidance, Navigation and Control Conference and Exhibit, 2007. 10.2514/6.2007-6813.

D. Choukroun, I. Y. Bar-Itzhack, and Y. Oshman. Optimal-request algorithm for attitude determination. Journal of Guidance, Control, and Dynamics, 27(3):418-425, 2004. 10.2514/1.10337.

J. A. Christian and E. G. Lightsey. Sequential optimal attitude recursion filter. Journal of Guidance, Control, and Dynamics, 33(6):1787-1800, 2010. 10.2514/1.49561.

J. Crassidis. Sigma-point kalman filtering for integrated gps and inertial navigation. IEEE Transactions on Aerospace and Electronic Systems, 42(2):750-756, 2006. 10.1109/taes.2006.1642588.

J. L. Crassidis, F. Landis Markley, and Y. Cheng. Survey of nonlinear attitude estimation methods. Journal of Guidance, Control, and Dynamics, 30(1):12-28, 2007a. ISSN 07315090. 10.2514/ 1.22452 .

J. L. Crassidis, F. L. Markley, and Y. Cheng. Survey of nonlinear attitude estimation methods. Journal of Guidance, Control, and Dynamics, 30(1):12-28, 2007b. 10.2514/1.22452.

H. G. De Marina, F. J. Pereda, J. M. Giron-Sierra, and F. Espinosa. UAV attitude estimation using unscented Kalman filter and TRIAD. IEEE Transactions on Industrial Electronics, 59 (11):4465-4474, 2012. ISSN 02780046. 10.1109/TIE.2011.2163913.

M. B. Del Rosario, N. H. Lovell, and S. J. Redmond. Quaternion-Based Complementary Filter for Attitude Determination of a Smartphone. IEEE Sensors Journal, 16(15):6008-6017, 2016. ISSN 1530437X. 10.1109/JSEN.2016.2574124.

G. Dong, X. Zhang, C. Zhang, and Z. Chen. A method for state of energy estimation of lithium-ion batteries based on neural network model. Energy, 90:879-888, 2015. 10.1016/j.energy.2015.07 .120 .

H. Eric Tseng, L. Xu, and D. Hrovat. Estimation of land vehicle roll and pitch angles. Vehicle System Dynamics, 45(5):433-443, 2007. 10.1080/00423110601169713.

J. L. Farrell, J. C. Stuelpnagel, R. H. Wessner, J. R. Velman, and J. E. Brook. A least squares estimate of satellite attitude (grace wahba). SIAM Review, 8(3):384-386, 1966. 10.1137/1008080.

K. Feng, J. Li, X. Zhang, C. Shen, Y. Bi, T. Zheng, and J. Liu. A new quaternion-based kalman filter for real-time attitude estimation using the two-step geometrically-intuitive correction algorithm. Sensors (Switzerland), 17(9), 2017. ISSN 14248220. 10.3390/s17092146.

X. Gao, Z. Yu, J. Neubeck, and J. Wiedemann. Sideslip angle estimation based on input-output linearisation with tire-road friction adaptation. Vehicle System Dynamics, 48(2):217-234, 2010. $10.1080 / 00423110902721824$.

N. Gordon, D. Salmond, and A. Smith. Novel approach to nonlinear/non-gaussian bayesian state estimation. IEE Proceedings F Radar and Signal Processing, 140(2):107, 1993. 10.1049/ip-f-2 .1993 .0015 .

H. F. Grip, L. Imsland, T. A. Johansen, J. C. Kalkkuhl, and A. Suissa. Vehicle sideslip estimation: Design, implementation, and experimental validation. IEEE Control Systems, 29(5):36-52, 2009. 10.1109/mcs.2009.934083.

H. Guo, D. Cao, H. Chen, C. Lv, H. Wang, and S. Yang. Vehicle dynamic state estimation: State of the art schemes and perspectives. IEEE/CAA Journal of Automatica Sinica, 5(2):418-431, 2018. ISSN 23299274. 10.1109/JAS.2017.7510811.

M. Gwak, K. Jo, and M. Sunwoo. Neural-network multiple models filter (nmm)-based position estimation system for autonomous vehicles. International Journal of Automotive Technology, 14 (2):265-274, 2013. 10.1007/s12239-013-0030-2. 
A. Hac and M. D. Simpson. Estimation of vehicle side slip angle and yaw rate. SAE Technical Papers, 2000(724), 2000. ISSN 26883627. 10.4271/2000-01-0696.

K. S. Hatamleh, M. Al-Shabi, A. Al-Ghasem, and A. A. Asad. Unmanned aerial vehicles parameter estimation using artificial neural networks and iterative bi-section shooting method. Applied Soft Computing, 36:457-467, 2015. 10.1016/j.asoc.2015.06.031.

P. C. Hughes. Spacecraft Attitude Dynamics. Dover Publications, 2012.

L. Imsland, T. A. Johansen, T. I. Fossen, H. Fjær Grip, J. C. Kalkkuhl, and A. Suissa. Vehicle velocity estimation using nonlinear observers. Automatica, 42(12):2091-2103, 2006. 10.1016/ j.automatica.2006.06.025.

L. Imsland, H. F. Grip, T. A. Johansen, T. I. Fossen, J. C. Kalkkuhl, and A. Suissa. Nonlinear observer for vehicle velocity with friction and road bank angle adaptation-validation and comparison with an extended kalman filter. Proc. Society of Automotive Engineers (SAE), Michigan, USA, 2007.

K. J. Jensen. Generalized nonlinear complementary attitude filter. Journal of Guidance, Control, and Dynamics, 34(5):1588-1593, 2011.

X. Jin, G. Yin, and N. Chen. Advanced estimation techniques for vehicle system dynamic state: A survey. Sensors (Switzerland), 19(19):1-26, 2019. ISSN 14248220. 10.3390/s19194289.

A. Jouybari, A. A. Ardalan, and M. H. Rezvani. Experimental comparison between Mahoney and Complementary sensor fusion algorithm for attitude determination by raw sensor data of Xsens IMU on buoy. International Archives of the Photogrammetry, Remote Sensing and Spatial Information Sciences - ISPRS Archives, 42(4W4):497-502, 2017. ISSN 16821750. 10.5194/ isprs-archives-XLII-4-W4-497-2017.

S. Julier and J. Uhlmann. Unscented filtering and nonlinear estimation. Proceedings of the IEEE, 92(3):401-422, 2004. 10.1109/jproc.2003.823141.

J. E. Keat. Analysis of least-squares attitude determination routine DOAOP. 1977.

W. Khalaf, I. Chouaib, and M. Wainakh. Novel adaptive UKF for tightly-coupled INS/GPS integration with experimental validation on an UAV. Gyroscopy and Navigation, 8(4):259-269, 2017. ISSN 20751109. 10.1134/S2075108717040083.

H. U. Kim and T. S. Bae. Deep learning-based GNSS network-based real-time kinematic improvement for autonomous ground vehicle navigation. Journal of Sensors, 2019, 2019. ISSN 16877268. $10.1155 / 2019 / 3737265$.

G. M. Lerner. Three-Axis Attitude Determination Methods, page 420-428. D. Reidel, 1978.

H. Li. Examples From Intelligent Vehicle Applications ( Part III ): Handling Nonlinear Estimation Problems And The Unscented Kalman Filter A Brief Tutorial On Recursive Estimation With Examples From Intelligent Vehicle Applications ( Part III ): Handling Nonline. (Part III), 2014.

Y. Liu, X. Jiang, and G. Ma. Marginalized particle filter for spacecraft attitude estimation from vector measurements. Journal of Control Theory and Applications, 5(1):60-66, 2007. 10.1007/ s11768-005-5294-2.

S. Ludwig, K. Burnham, A. Jimenez, and P. Touma. Comparison of attitude and heading reference systems using foot mounted MIMU sensor data: basic, Madgwick, and Mahony. page 96, 2018. ISSN 1996756X. 10.1117/12.2296568.

S. A. Ludwig and K. D. Burnham. Comparison of Euler Estimate using Extended Kalman Filter, Madgwick and Mahony on Quadcopter Flight Data. 2018 International Conference on Unmanned Aircraft Systems, ICUAS 2018, pages 1236-1241, 2018. 10.1109/ICUAS.2018.8453465. 
S. O. Madgwick, A. J. Harrison, and R. Vaidyanathan. Estimation of IMU and MARG orientation using a gradient descent algorithm. IEEE International Conference on Rehabilitation Robotics, pages 1-7, 2011. ISSN 19457898. 10.1109/ICORR.2011.5975346.

R. Mahony, T. Hamel, and J. M. Pflimlin. Nonlinear complementary filters on the special orthogonal group. IEEE Transactions on Automatic Control, 53(5):1203-1218, 2008. ISSN 00189286. 10.1109/TAC.2008.923738.

R. Mahony, T. Hamel, J. Trumpf, and C. Lageman. Nonlinear attitude observers on so(3) for complementary and compatible measurements: A theoretical study. Proceedings of the $48 \mathrm{~h}$ IEEE Conference on Decision and Control (CDC) held jointly with 2009 28th Chinese Control Conference, 2009.

P. Marantos, Y. Koveos, and K. J. Kyriakopoulos. Uav state estimation using adaptive complementary filters. IEEE Transactions on Control Systems Technology, 24(4):1214-1226, 2016.

J. L. Marins, X. Yun, E. R. Bachmann, R. B. McGhee, and M. J. Zyda. An extended Kalman filter for quaternion-based orientation estimation using MARG sensors. IEEE International Conference on Intelligent Robots and Systems, 4:2003-2011, 2001. 10.1109/iros.2001.976367.

F. Markley. Attitude determination and parameter estimation using vector observations. Journal of the Astronautical Sciences, 36(6):245-258, 1988. 10.2514/6.1988-4225.

F. Markley. Attitude determination and parameter estimation using vector observations: Theory. Journal of the Astronautical Sciences, 37(1):41-58, 1989. 10.2514/6.1988-4225.

F. Markley. Attitude determination and parameter estimation using vector observations: Application. Journal of the Astronautical Sciences, 39(3):367-381, 1991.

F. Markley. Attitude determination using vector observations: a fast optimal matrix algorithm. Journal of the Astronautical Sciences, 41(2):261-280, 1993.

F. L. Markley and D. Mortari. How to estimate attitude from vector observations. Advances in the Astronautical Sciences, 103(PART III):1979-1996, 2000. ISSN 00653438.

P. Martin and E. Salaün. Generalized Multiplicative Extended Kalman Filter for Aided Attitude and Heading Reference System. 10.2514/6.2010-8300.

R. Mehra. Approaches to adaptive filtering. IEEE Transactions on Automatic Control, 17(5): 693-698, 1972 .

D. Mortari. Esoq: A closed-form solution to the wahba problem. Journal of the Astronautical Sciences, 45(2):195-204, 1997.

M. W. Mueller and R. D'Andrea. Stability and control of a quadrocopter despite the complete loss of one, two, or three propellers. Proceedings - IEEE International Conference on Robotics and Automation, pages 45-52, 2014. ISSN 10504729. 10.1109/ICRA.2014.6906588.

M. W. Mueller and R. D'Andrea. Relaxed hover solutions for multicopters: Application to algorithmic redundancy and novel vehicles. International Journal of Robotics Research, 35(8): 873-889, 2016. ISSN 17413176. 10.1177/0278364915596233.

NCEI. Magnetic declination, models, data and services - ncei, 2020. URL https://www.ngdc .noaa.gov/geomag/geomag. shtml.

M. Nguyen-H and C. Zhou. Improving gps/ins integration through neural networks, 2010.

M. Oudghiri, M. Chadli, and A. El Hajjaji. Lateral vehicle velocity estimation using fuzzy sliding mode observer,. Proc. Mediterranean Conf. Control and Automation, pages 1-6, 2007.

M. L. Psiaki. Attitude-determination filtering via extended quaternion estimation. Journal of Guidance, Control, and Dynamics, 23(2):206-214, 2000. 10.2514/2.4540. 
M. L. Psiaki. Backward-smoothing extended kalman filter. Journal of Guidance, Control, and Dynamics, 28(5):885-894, 2005. 10.2514/1.12108.

M. L. Psiaki. Generalized wahba problems for spinning spacecraft attitude and rate determination. The Journal of the Astronautical Sciences, 57(1-2):73-92, 2010. 10.1007/bf03321495.

R. Rajamani, D. Piyabongkarn, V. Tsourapas, and J. Y. Lew. Parameter and state estimation in vehicle roll dynamics. IEEE Transactions on Intelligent Transportation Systems, 12(4):15581567, 2011. 10.1109/tits.2011.2164246.

M. Roth and F. Gustafsson. An efficient implementation of the second order extended Kalman filter. 2011.

K. Saadeddin, M. F. Abdel-Hafez, M. A. Jaradat, and M. A. Jarrah. Performance enhancement of low-cost, high-accuracy, state estimation for vehicle collision prevention system using ANFIS. Mechanical Systems and Signal Processing, 41(1-2):239-253, 2013a. ISSN 08883270. 10.1016/ j.ymssp.2013.06.013. URL http://dx.doi.org/10.1016/j.ymssp.2013.06.013.

K. Saadeddin, M. F. Abdel-Hafez, M. A. Jaradat, and M. A. Jarrah. Performance enhancement of low-cost, high-accuracy, state estimation for vehicle collision prevention system using anfis. Mechanical Systems and Signal Processing, 41(1-2):239-253, 2013b. 10.1016/j.ymssp.2013.06 .013 .

A. M. Sabatini. Quaternion-based extended Kalman filter for determining orientation by inertial and magnetic sensing. IEEE Transactions on Biomedical Engineering, 53(7):1346-1356, 2006. ISSN 00189294. 10.1109/TBME.2006.875664.

A. M. Sabatini. Estimating three-dimensional orientation of human body parts by inertial/magnetic sensing. Sensors, 11(2):1489-1525, 2011. ISSN 14248220. 10.3390/s110201489.

S. Särkkä. On unscented Kalman filtering for state estimation of continuous-time nonlinear systems. IEEE Transactions on Automatic Control, 52(9):1631-1641, 2007. ISSN 00189286. 10.1109/ TAC.2007.904453.

M. D. Shuster. A survey of attitude representation. Journal of the Astronautical Sciences, 41(4): 439-517, 1993.

M. D. Shuster. The generalized wahba problem. The Journal of the Astronautical Sciences, 54(2): 245-259, 2006. 10.1007/bf03256485.

M. D. Shuster. Filter quest or request. Journal of Guidance, Control, and Dynamics, 32(2): 643-645, 2009. 10.2514/1.40423.

M. D. Shuster and S. D. Oh. Three-axis attitude determination from vector observation. Journal of Guidance, Control, and Dynamics, 4(1):70-77, 1981.

D. Simon. Optimal State Estimation: Kalman, H, and Nonlinear Approaches. 2006. ISBN 0471708585. 10.1002/0470045345.

H. Singer. Continuous-Discrete Unscented Kalman Filtering. pages 1-28, 2006.

Y. Song and J. W. Grizzle. The extended kalman filter as a local asymptotic observer for discretetime nonlinear systems. Estimation and Control, 5(1):59-78, 1995.

J. Stephant, A. Charara, and D. Meizel. Virtual sensor: Application to vehicle sideslip angle and transversal forces. IEEE Transactions on Industrial Electronics, 51(2):278-289, 2004. 10.1109/ tie.2004.824857.

J. Stuelpnagel. On the parametrization of the three-dimensional rotation group. SIAM Review, 6 (4):422-430, 1964. 10.1137/1006093. 
S. Sun, R. J. Schilder, and C. C. De Visser. Identification of quadrotor aerodynamic model from high speed flight data. AIAA Atmospheric Flight Mechanics Conference, 2018, (209999), 2018a. $10.2514 / 6.2018-0523$.

S. Sun, L. Sijbers, X. Wang, and C. De Visser. High-Speed Flight of Quadrotor Despite Loss of Single Rotor. IEEE Robotics and Automation Letters, 3(4):3201-3207, 2018b. ISSN 23773766. 10.1109/LRA.2018.2851028.

Y. Tian, H. Wei, and J. Tan. An adaptive-gain complementary filter for real-time human motion tracking with MARG sensors in free-living environments. IEEE Transactions on Neural Systems and Rehabilitation Engineering, 21(2):254-264, 2013. ISSN 15344320. 10.1109/TNSRE.2012 .2205706 .

S. Vathsal. Spacecraft attitude determination using a second-order nonlinear filter. Journal of Guidance, Control, and Dynamics, 10(6):559-566, 1987. 10.2514/3.20256.

G. Wahba. Problem 65-1: A least squares estimate of satellite attitude. SIAM Review, 7(3):409, 1966.

E. A. Wan, R. van der Merwe, and S. I. Julier. Sigma-point Kalman filters for nonlinear estimation and sensor fusion applications to integrated navigation. 2004.

W. Wei, B. Shaoyi, Z. Lanchun, Z. Kai, W. Yongzhi, and H. Weixing. Vehicle Sideslip Angle Estimation Based on General Regression Neural Network. Mathematical Problems in Engineering, 2016, 2016. ISSN 15635147. 10.1155/2016/3107910.

R. B. Widodo and C. Wada. Attitude Estimation Using Kalman Filtering: External Acceleration Compensation Considerations. Journal of Sensors, 2016, 2016. ISSN 16877268. 10.1155/2016/ 6943040 .

D. R. Williams. Earth fact sheet, 2020. URL https://nssdc.gsfc.nasa.gov/planetary/ factsheet/earthfact.html.

J. Wu, Z. Zhou, J. Chen, H. Fourati, and R. Li. Fast Complementary Filter for Attitude Estimation Using Low-Cost MARG Sensors. IEEE Sensors Journal, 16(18):6997-7007, 2016. ISSN 1530437X. 10.1109/JSEN.2016.2589660.

Y. Xiaoping, C. Aparicio, E. R. Bachmann, and R. B. McGhee. Implementation and experimental results of a quaternion-based Kalman filter for human body motion tracking. Proceedings - IEEE International Conference on Robotics and Automation, 2005(6):317-322, 2005. ISSN 10504729. 10.1109/ROBOT.2005.1570138.

X. Yuan, S. Yu, S. Zhang, G. Wang, and S. Liu. Quaternion-based unscented kalman filter for accurate indoor heading estimation using wearable multi-sensor system. Sensors (Switzerland), 15(5):10872-10890, 2015. ISSN 14248220. 10.3390/s150510872.

Z. Q. Zhang, X. L. Meng, and J. K. Wu. Quaternion-based kalman filter with vector selection for accurate orientation tracking. IEEE Transactions on Instrumentation and Measurement, 61 (10):2817-2824, 2012. ISSN 00189456. 10.1109/TIM.2012.2196397.

L.-H. Zhao, Z.-Y. Liu, and H. Chen. Design of a nonlinear observer for vehicle velocity estimation and experiments. IEEE Transactions on Control Systems Technology, 19(3):664-672, 2011. 10.1109/tcst.2010.2043104. 
Part II

Scientific Paper 



\title{
Attitude Estimation of a Quadcopter with one fully damaged rotor using on-board MARG Sensors
}

\author{
Dr ir C.C. de Visser, Sihao Sun, and Prashant Solanki \\ Delft University of Technology, 2629 HS Delft, The Netherlands
}

\begin{abstract}
Quadcopters are becoming increasingly popular across diverse sectors such as mapping, photography, or surveillance. Since rotor damages occur frequently, it is essential to improve the attitude estimation and thus ultimately the ability to control a damaged quadcopter. The Control and Simulation group of TU Delft developed a quadcopter controller for the case of a single damaged rotor in an inside environment, where the attitude and position of the quadcopter are provided by an external system. In the present research, a novel attitude estimator called Adaptive Fuzzy Complementary Kalman Filter (AFCKF) has been developed and validated that works independently of any external systems. It is able to estimate the attitude of a quadcopter with one fully damaged rotor while only relying on the on-board sensors. The results show that the AFCKF is able to provide significantly better attitude estimates for flights with a damaged rotor than mainstream filters, estimating the roll and pitch of the quadcopter with an RMS error of less than 1.7 degrees and a variance of less than 2 degrees. It is the only filter that is able to track the quadcopter's yaw angle for the case of a damaged rotor, while showing only a comparatively small rise in the computational cost.
\end{abstract}




\section{Introduction}

By virtue of mechanical simplicity, quadcopters have become very popular in multiple industries such as delivery, surveillance, photography, mapping, and more. Although cheap and versatile in nature, quadcopters suffer from the inherent problem of a lack of redundancy in the control surface (rotors). A wide range of research has been conducted to develop a fault-tolerant controller for a quadcopter subjected to rotor failure (Mueller and D'Andrea (2014), Mueller and D'Andrea (2016), Sun et al. (2018b)). In the scenario of a complete single rotor failure, the quadcopter has to abandon yaw control and spin at a high angular rate. This motion improves the stability of the system due to the gyroscopic effect, but deteriorates the position and attitude estimation. Rotor damages occur frequently, which is why research that improves the attitude estimation and thus ultimately the ability to control a damaged quadcopter is of high importance in the field.

The Control and Simulation group of TU Delft has developed a fault-tolerant controller and successfully conducted an indoor flight of a quadcopter subjected to a complete single rotor failure (Sun et al. (2018b)). The attitude of the quadcopter was attained using a motion-capture system that provides the system's/vehicle's attitude in order to test the controller. Unfortunately, no such motion capture system exists for the outside environment. Thus, the objective of the present research is to develop an attitude estimation method that can estimate the states of the quadcopter with a damaged rotor with sufficient accuracy while using only the on-board sensors (magnetometer, accelerometer, and rate gyroscope, or 'MARG' sensors) and is consequently suitable for an outside environment.

The quadcopter's on-board sensors have a variety of problems that makes the task of attitude estimation more complicated. The accelerometer measures the specific force that is applied to the sensor. In an ideal situation, the accelerometer is situated at the center of mass of the quadcopter, but in reality that is not possible. Furthermore, the accelerometer readings suffer from high noise, bias issues, and other external accelerations. The rate gyroscope measures the rotation velocity of the quadcopter, which under ideal circumstances can be integrated (provided the initial conditions are known) to prove the current attitude estimates (Bortz (1971), Ignagni (1990)). In low-cost applications, microelectromechanical systems (MEMS) gyroscopes are used because of their low cost and light weight (Yazdi et al. (1998)). These sensors suffer from high random noise and are furthermore affected by time-varying bias and gyroscopic drift. The magnetometer measures the local magnetic field. In an ideal situation, it is identical to the earth's local magnetic field, which is known, but in reality, the measured reading is corrupted with local magnetic disturbance, noise, and bias-related issues.

There exist a wide spectrum of attitude estimation techniques based on different approaches to cater for the problem of attitude estimation using on-board MARG sensors. There are multiple stochastic estimation techniques which are based on Kalman Filters or Extended Kalman Filters (EKF), such as Foxlin (1996), Marins et al. (2001), Xiaoping et al. (2005), and Wang et al. 
(2015). The issue with Kalman-based estimators is that they tend to be computationally heavy and are affected by linearization errors. Furthermore, the magnetic distortions and the external accelerations of the quadcopter adversely affect the attitude estimates. Another approach is based on Wahba's problem (Wahba (1966)), which inspired filters such as Markley (1988), Mortari (1997), Shuster and Oh (1981), and Christian and Lightsey (2010). However, although they do not need to be initiated, these filters tend to be highly susceptible to noise, which means that applying them on low-cost sensors can lead to high estimation errors. Additionally, just like the Kalman Filter-based methods, the magnetic distortions and external accelerations of the quadcopter also adversely affect the attitude estimates. Lastly, complementary filter based methods such as Madgwick et al. (2011), Mahony et al. (2008), Baerveldt and Klang (1997), Tian et al. (2013), and Calusdian et al. (2011) tend to be computationally lighter and are developed for low-cost MARG sensors, but they too are adversely affected by magnetic distortions and external accelerations.

Consequently, none of these attitude estimation techniques is able to perform sufficiently well under highly dynamic motion such as is the case when one rotor is fully damaged and the quadcopter rotates about the yaw axis, which causes the accelerometer to measure centrifugal acceleration because it is not placed exactly at the center of rotation. The present research intends to fill that gap. There are a number of recent attitude estimators that can cater for short-term external accelerations and magnetic distortions, such as Del Rosario et al. (2016), Wu et al. (2016), Yuan et al. (2015), Feng et al. (2017), and Sabatini (2006). However, if there are long-term external accelerations or magnetic distortions, such as when the quadcopter rotates about the yaw axis, these filters are not able to provide satisfactory attitude estimates.

The main contribution of this paper is that it overcomes these obstacles and is able to accurately estimate the attitude of the quadcopter under highly dynamic conditions, as in the case of a fully damaged rotor. The Adaptive Fuzzy Complementary Kalman Filter (AFCKF) developed here is designed in such a way that the yaw estimate is obtained through a channel separate from the channels of the roll and pitch estimates. Furthermore, the accelerometer readings are compensated for centrifugal acceleration using a simple model scheme. The AFCKF is a generic filter that can be used for attitude estimation given a MARG for any system, although it has been developed with the mindset of a quadcopter. The AFCKF is a combination of an EKF and a complementary filter which is able to provide accurate attitude estimates while keeping the computational cost low. Unlike traditional filters, the filter gains are adaptive in nature and are adjusted based on different criteria.

To validate the attitude estimator, its estimates are compared with the estimates obtained via the OptiTrack motion capture system that are extremely close to the real attitude of the quadcopter. To measure the performance of the AFCKF relative to existing attitude estimation techniques used for quadcopters, it is compared to the three most popular mainstream attitude estimators, namely the one developed by Madgwick et al. (2011) (from here on called 'Madgwick 
Filter'), the one developed by Valenti et al. (2015) (from here on called 'Complementary Filter'), and the one developed by Roetenberg et al. (2005) (from here on called 'Kalman Filter'). The comparison is undertaken for four different cases: Hover flight with and without damaged rotor as well as motion flight with and without damaged rotor. As the results show, the AFCKF is able to estimate the quadcopter's attitude as accurate as the other filters for the cases of non-damaged rotor flights, and has a significant better performance than the other three filters for the cases in which the rotor is damaged. For the latter case, the AFCKF estimates the roll and pitch of the quadcopter with an RMS error of less than 1.7 degrees and a variance of less than 2 degrees. For the yaw estimate, although it can be seen that as the yaw rate increases, the estimation error increases as well, it is the only filter that is able to track the quadcopter's yaw angle.

For the case without damaged rotor, the AFCKF has an RMS yaw error of less than 2.5 degrees and roll and pitch RMS errors of about 2 degrees. It has a variance of less than 2.3 degrees (yaw) and less than 1.4 degrees (roll and pitch) respectively. Although the Madgwick Filter consistently shows the lowest execution time and is on average 1.4 times faster than the AFCKF, the AFCKF is still on average five times faster than the EKF-based filter and about six times faster than the complex complementary filter.

The AFCKF is primarily developed in the quaternion representation scheme, which will be explained in the following chapter. After that, the filter will be derived in detail in chapter three. Chapter four shows and discusses the results of the experiments and the comparison with other filters, which is followed by a conclusion and a look at possible future works in chapter five.

\section{Quaternion representation scheme}

In order to develop an attitude estimator for a quadcopter, its orientation with respect to a certain frame needs to be known. The attitude or orientation of a system can be represented in multiple ways. There exist a variety of parameterisation schemes, such as Euler angles, quaternions, Rodrigues parameters, or modified Rodrigues parameters, which each have different benefits and drawbacks. Stuelpnagel (1964), Shuster (1993) and Hughes (2012) provide a more detailed insight into different ways of representing attitude. The AFCKF is based on the quaternion representation of the attitude, which is chosen because it does not suffer form singularity issues like the Euler representation, and because the mathematical interpolation of a quaternion is simpler. 


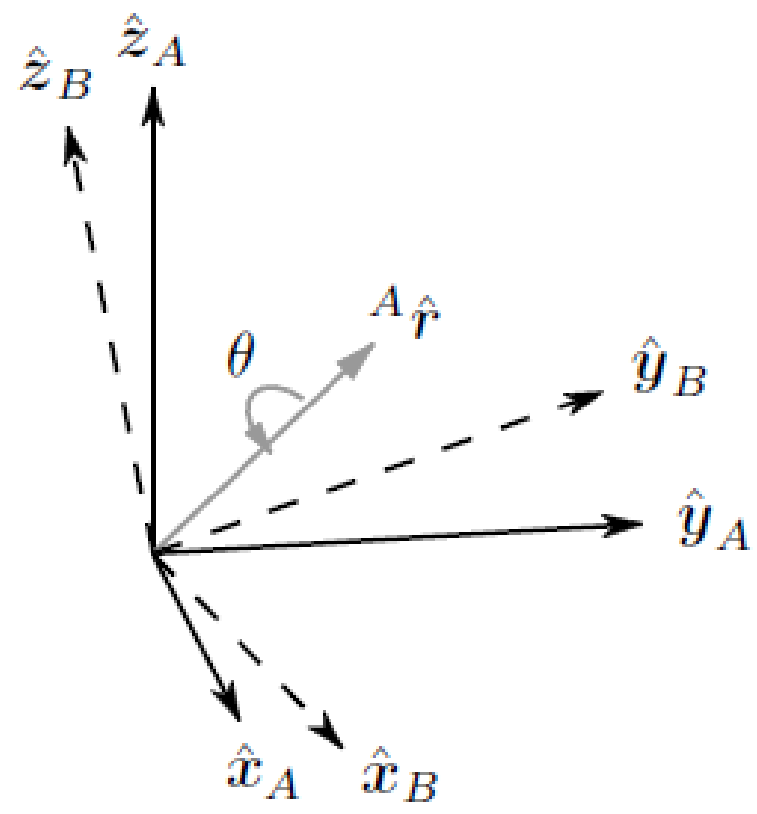

Figure 2.1: Frame B orientation achieved via rotating Frame A

A quaternion is a four-dimensional number. Quaternions are used to describe the orientation of a frame with respect to another frame: A reference frame $A$ can be rotated about an axis (unit axis) in frame $A\left(r_{A}\right)$ by an angle theta $(\theta)$ to transform frame $A$ into frame $B$, provided that both the frames follow either left- or right-hand notation. Thus $q_{A / B}$ provides the orientation of the frame $B$ with respect to frame $A$. The quaternion is defined as shown in equation 1 , with $r_{A}$ being a unit vector as shown by equation 2 .

$$
\begin{gathered}
q_{A / B}=\left[\begin{array}{c}
q_{1} \\
q_{2} \\
q_{3} \\
q_{4}
\end{array}\right]^{T}=\left[\begin{array}{c}
\cos (\theta / 2) \\
-r_{x} \sin (\theta / 2) \\
-r_{y} \sin (\theta / 2) \\
-r_{z} \sin (\theta / 2)
\end{array}\right]^{T} \\
r_{A}^{2}=r_{x}^{2}+r_{y}^{2}+r_{z}^{2}
\end{gathered}
$$

Furthermore, quaternions are constrained to be normalised as shown in equation 3, where $q^{T}$ is the quaternion transpose.

$$
q q^{T}=q_{1}^{2}+q_{2}^{2}+q_{3}^{2}+q_{4}^{2}=1
$$

The quaternion conjugate, denoted with ${ }^{\prime} *^{\prime}$ on the superscript, describes the orientation of frame 
$A$ with respect to frame $B$ and is given by equation 4 .

$$
q_{B / A}=q_{A / B}^{*}=\left[\begin{array}{c}
q_{1} \\
-q_{2} \\
-q_{3} \\
-q_{4}
\end{array}\right]^{T}
$$

The quaternion product is used to find the compound orientation. If two quaternions $q_{B / C}$ and $q_{A / B}$ are known, then $q_{A / C}$ can be found using equation 5 (Kuipers (1999)). The quaternion product can be obtained using the Hamilton theorem, given by equation 6 . The quaternion product is not commutative, i.e., $q_{B / C} \otimes q_{A / B} \neq q_{A / B} \otimes q_{B / C}$.

$$
\begin{gathered}
q_{A / C}=q_{B / C} \otimes q_{A / B} \\
p \otimes r=\left[\begin{array}{c}
p_{1} \\
p_{1} \\
p_{3} \\
p_{4}
\end{array}\right]^{T}\left[\begin{array}{l}
r_{1} \\
r_{2} \\
r_{3} \\
r_{4}
\end{array}\right]^{T}=\left[\begin{array}{l}
p_{1} r_{1}-p_{2} r_{2}-p_{3} r_{3}-p_{4} r_{4} \\
p_{1} r_{2}+p_{2} r_{1}+p_{3} r_{4}-p_{4} r_{3} \\
p_{1} r_{3}-p_{2} r_{4}+p_{3} r_{1}+p_{4} r_{2} \\
p_{1} r_{4}+p_{2} r_{3}-p_{3} r_{2}+p_{4} r_{1}
\end{array}\right]^{T}
\end{gathered}
$$

If $q_{A / B}$ is known, then a three-dimensional vector in frame $A\left(\vec{v}^{A}\right)$ can be converted into frame $B$ using equation 7. An additional zero is added as the fourth element of the vector in frame $A\left(\vec{v}^{A}\right)$ and in frame $B\left(\vec{v}^{B}\right)$.

$$
\left[\begin{array}{ll}
0 & \vec{v}^{B}
\end{array}\right]=q_{A / B} \otimes\left[\begin{array}{ll}
0 & \vec{v}^{A}
\end{array}\right] \otimes q_{A / B}^{*}
$$

Equation 7 can be represented in the form of a rotation matrix $\left(C_{B / A}\right)$ as shown in equation 8 (Kuipers (1999)), where $C_{B / A}$ is the rotation matrix that transforms the vectors in frame $A$ into the vectors in frame $B$.

$$
C_{B / A}=\left[\begin{array}{ccc}
-1+2\left(q_{1}^{2}+q_{2}^{2}\right) & 2\left(q_{2} q_{3}+q_{4} q_{1}\right) & 2\left(q_{2} q_{4}-q_{3} q_{1}\right) \\
2\left(q_{2} q_{3}-q_{4} q_{1}\right) & -1+2\left(q_{1}^{2}+q_{3}^{2}\right) & 2\left(q_{3} q_{4}+q_{2} q_{1}\right) \\
2\left(q_{2} q_{4}+q_{3} q_{1}\right) & 2\left(q_{3} q_{4}-q_{2} q_{1}\right) & -1+2\left(q_{1}^{2}+q_{4}^{2}\right)
\end{array}\right]
$$

The relationship between the quaternion Euler angles, i.e., roll angle $(\phi)$, pitch angle $(\theta)$ and yaw angle $(\psi)$, is given by equation 9 .

$$
\begin{gathered}
\phi=\tan ^{-1}\left\{\frac{2\left(q_{3} q_{2}-q_{1} q_{4}\right)}{1-2 q_{1}^{2} 2 q_{2}^{2}}\right\} \\
\theta=\sin ^{-1}\left\{2\left(q_{2} q_{4}+q_{1} q_{3}\right)\right\} \\
\psi=\tan ^{-1}\left\{\frac{2\left(q_{2} q_{3}-q_{1} q_{2}\right)}{2 q_{1}^{2} 2 q_{4}^{2}-1}\right\}
\end{gathered}
$$


The attitude quaternion is later converted into Euler angles using equation 9. The reasons for this are twofold: Firstly, it is easier to understand the orientation of the system if represented in Euler angles - it is more intuitive - and secondly, the fault tolerant controller is based on Euler representation.

\section{Filter Derivation}

In this section, the filter is derived and presented along with the equations of the attitude estimator. The schematics of the AFCKF are shown in figure 3.1. For a better understanding, the AFCKF is divided into five parts, as can be seen in the attitude estimator schematics. Part one describes the removal of the external accelerations from the accelerometer readings, which leads to these readings being dominated by the gravity vector. Part two constitutes the gradient descent algorithm that utilises the corrected accelerometer readings to obtain a set of the quadcopter's attitude estimates. Part three consists of the Kalman filter that uses the gyroscope readings and attitude estimates obtained from part two in order to provide the optimal roll and pitch estimates. Part four comprises a gyroscope bias drift correction method based on Mahony et al. (2008). Lastly, in part five, the yaw estimate is obtained by processing the gyroscope and magnetometer readings through a fuzzy linear complementary filter. 


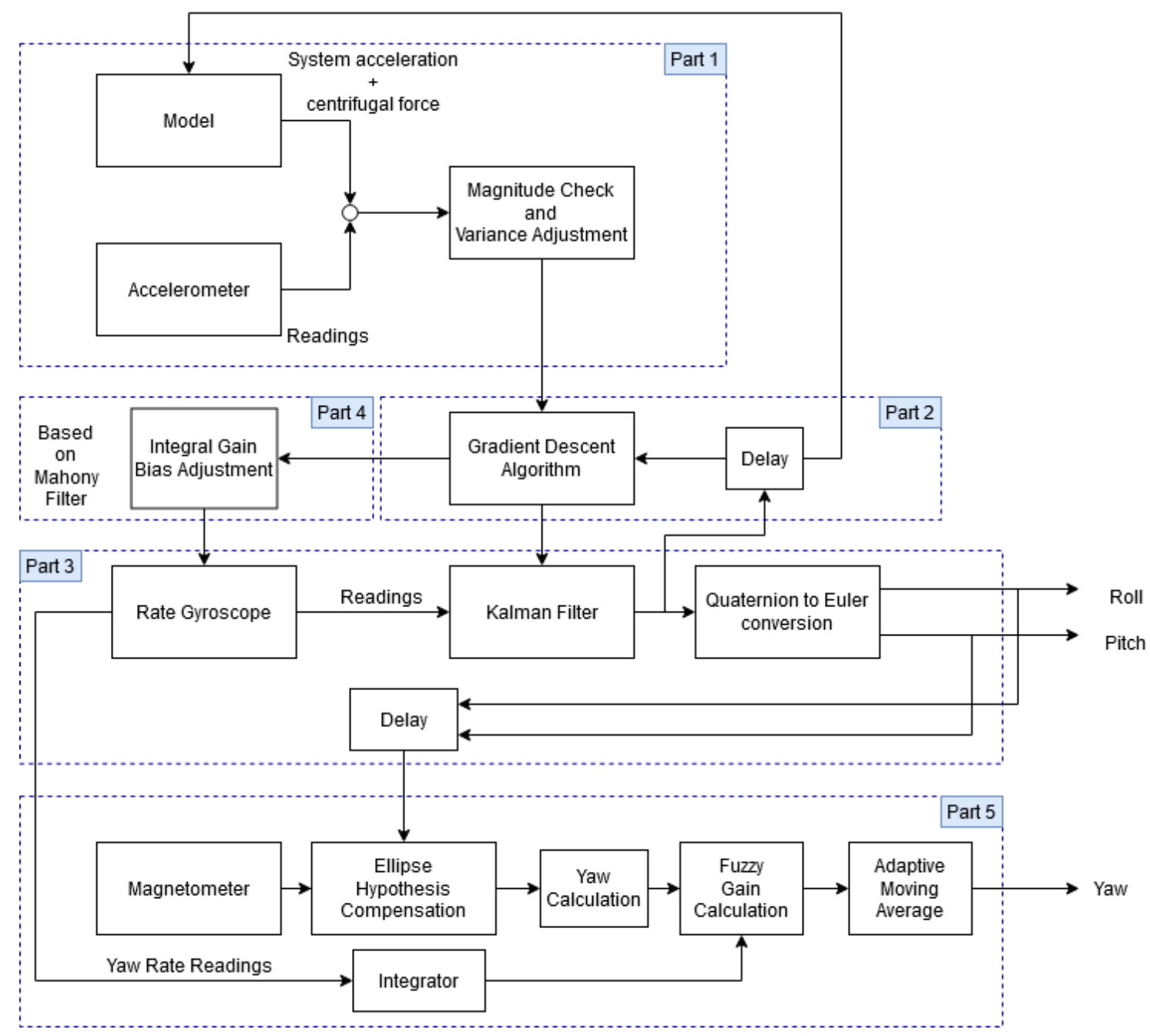

Figure 3.1: Schematics of the AFCKF

\subsection{Correcting the accelerometer readings}

The accelerometer measures the specific force applied to the sensor in the body frame of reference (Titterton and Weston (2004)). Thus, the ideal measurement model of an accelerometer in space is given by equation 10 , where $\vec{a}_{B}$ is the acceleration of the body, $\vec{r}_{B}$ is the position vector of the accelerometer from the center of rotation, $\vec{\omega}_{B}$ is the rotational velocity of the body reference frame, $2 \vec{\omega}_{B} \times \dot{\vec{r}}_{B}$ is the Coriolis acceleration (which is zero in the case of a fixed sensor, because the radius from the center of rotation stays constant), $\vec{g}_{B}$ is the gravity vector in the body frame of reference, $\vec{\omega}_{B} \times\left(\vec{\omega}_{B} \times \vec{r}_{B}\right)$ is the centrifugal acceleration and $\dot{\vec{\omega}}_{B} \times \vec{r}_{B}$ is zero under the assumption that $\dot{\vec{\omega}}_{B}$ is zero for a quadcopter, i.e., the rotational acceleration of the quadcopter is zero. Furthermore, the accelerometer readings are corrupted with noise denoted by $e_{a}$ and bias denoted by $\delta_{a, t}$. The noise is assumed to be zero mean white Gaussian noise with a standard deviation $\Sigma_{a}$, as given by equation 12. Equation 10 reduces to 11 due to the aforementioned reasons. 


$$
\begin{gathered}
\vec{a}_{B_{\text {measured }}}=\vec{a}_{B}+\dot{\vec{\omega}}_{B} \times \vec{r}_{B}+2 \vec{\omega}_{B} \times \dot{\vec{r}}_{B}+\vec{\omega}_{B} \times\left(\vec{\omega}_{B} \times \vec{r}_{B}\right)+\vec{g}_{B} \\
\vec{a}_{B_{\text {measured }}}=\vec{a}_{B}+\vec{\omega}_{B} \times\left(\vec{\omega}_{B} \times \vec{r}_{B}\right)+\vec{g}_{B}+e_{a}+\delta_{a, t} \\
\Sigma_{a}=\left[\begin{array}{ccc}
\sigma_{a c c_{x}} & 0 & 0 \\
0 & \sigma_{a c c_{y}} & 0 \\
0 & 0 & \sigma_{a c c_{z}}
\end{array}\right]
\end{gathered}
$$

To obtain the attitude estimate of the quadcopter using the accelerometer readings, the accelerometer readings are aligned with the gravity vector. Thus, in order to obtain correct attitude estimates, the accelerometer readings must be dominated by the gravity vector. However, the quadcopter's linear acceleration $\left(\vec{a}_{B}\right)$ and the centrifugal acceleration $\left(\vec{\omega}_{B} \times\left(\vec{\omega}_{B} \times \vec{r}_{B}\right)\right)$ are also measured by the accelerometer (the latter because the accelerometer is not exactly placed at the center of rotation), and are considered to be external noise and thus need to be accounted for. These external noises are modeled using predetermined models. The estimator is designed in such a way that only a crude model of the centrifugal force about the z-axis is required, which is given by equation 13, where $\omega_{z}^{2}$ is the yaw rate of the quadcopter, and $\varpi_{1}$ and $\varpi_{2}$ are constants. The value of these constants is determined and shown in appendix B. The centrifugal force about only the body frame z-axis is modeled because the controller developed by Sun et al. (2018b) rotates about the body frame yaw axis after the rotor damage occurs.

$$
\vec{a}_{C_{B}}=\left[\begin{array}{lll}
\vec{F}_{C_{B x}} & \vec{F}_{C_{B y}} & \vec{F}_{C_{B_{z}}}
\end{array}\right]=\omega_{z}^{2} \cdot\left[\begin{array}{lll}
\varpi_{1} & \varpi_{2} & 0
\end{array}\right]
$$

The developed model is used to compensate for the centrifugal acceleration. Furthermore, it is assumed that the linear acceleration of the quadcopter is short-lived and small compared to the acceleration due to gravity. Thus, after the centrifugal acceleration is compensated for, the measured acceleration is given by equation 14. The obtained accelerometer reading is normalised against the gravity vector as given by equation 15 , where $g$ is the acceleration due to gravity.

$$
\begin{gathered}
\vec{a}_{B_{\text {corrected }}}=\vec{a}_{B_{\text {measured }}-\vec{a}_{C_{B}}} \\
\vec{a}_{\text {cor }}=\frac{\vec{a}_{B_{\text {corrected }}}}{|g|}
\end{gathered}
$$

\subsection{Obtaining the attitude from the accelerometer reading}

As explained in the previous section, the accelerometer readings are corrected using a model to remove the external acceleration from the readings. Thus it is safe to assume that the vector $\vec{a}_{B_{\text {corrected }}}$ obtained after correcting the accelerometer reading is dominated by the gravity vector, 
i.e., vector $\vec{a}_{B}$ is the measurement of the gravity vector in the quadcopter body reference frame. If magnitude and direction of a field in the earth frame are known, the attitude of the body frame relative to the earth can be calculated using an observation of the corresponding field in the sensor/body frame. However, the calculated orientation is not unique. There exist infinite solutions represented by rotating the true attitude about an axis parallel to the direction of the field in the earth frame. Thus, since only accelerometer readings are used to obtain an attitude estimate, the corresponding yaw estimates cannot be trusted (because the gravity vector runs parallel to the earth frame's yaw axis).

This attitude estimation problem can be converted into an optimisation problem where a rotation operation, given by equation 7 , aligns the known field in the earth frame, $\hat{v}$, to the corresponding measurement of the field in the sensor frame, $\hat{s}$, thus providing the attitude of the sensor with respect to the inertial frame. An optimisation algorithm scheme is formulated to acquire an attitude estimate. The minimisation function is given by equation 16 .

$$
\begin{gathered}
\min [f(q, \hat{v}, \hat{s})] \\
f(q, \hat{v}, \hat{s})=q_{t-1} \otimes \hat{v} \otimes q_{t-1}-\hat{s} \\
\hat{v}=\left[\begin{array}{llll}
0 & v_{x} & v_{y} & v_{z}
\end{array}\right] \\
\hat{s}=\left[\begin{array}{llll}
0 & s_{x} & s_{y} & s_{z}
\end{array}\right]
\end{gathered}
$$

The gradient descent algorithm is used for optimising and obtaining an attitude estimate. This method is chosen for the purpose of optimisation because of its low computational cost and simplicity. The algorithm is given by equations 17 and 18, used for obtaining the attitude estimate $q_{n}$, given the initial estimate $q_{0}$, where $n$ is the number of iterations, $\hat{v}$ is the field vector in the earth frame with an added null dimension, $\hat{s}$ consists of the measurement of the field in the sensor frame with an added null dimension, and $\mu$ is the step size. The optimal value of the step size ensures the convergence of the $q_{k}$. Equation 19 and 20 give the gradient, defined by the minimisation function and its Jacobian respectively.

$$
\begin{gathered}
q_{k}=q_{k-1}-\mu \frac{\nabla f\left(q_{k-1}, v, s\right)}{\left\|\nabla f\left(q_{k-1}, v, s\right)\right\|}, k=1,2 \ldots n \\
\nabla f\left(q_{k-1}, v, s\right)=J^{T}\left(q_{k-1}, v\right) f\left(q_{k-1}, v, s\right) \\
f\left(q_{k-1}, v, s\right)=\left[\begin{array}{l}
2 v_{x}\left(\frac{1}{2}-q_{3}^{2}-q_{4}^{2}\right)+2 v_{y}\left(q_{1} q_{4}+q_{2} q_{3}\right)+2 v_{z}\left(q_{2} q_{4}-q_{1} q_{3}\right)-s_{x} \\
2 v_{x}\left(q_{2} q_{3}-q_{1} q_{4}\right)+2 v_{y}\left(\frac{1}{2}-q_{2}^{2}-q_{4}^{2}\right)+2 v_{z}\left(q_{2} q_{1}+q_{4} q_{3}\right)-s_{y} \\
2 v_{x}\left(q_{3} q_{1}+q_{4} q_{2}\right)+2 v_{y}\left(q_{3} q_{4}-q_{1} q_{2}\right)+2 v_{z}\left(\frac{1}{2}-q_{2}^{2}-q_{3}^{2}\right)-s_{z}
\end{array}\right]
\end{gathered}
$$


$J\left(q_{k-1}, v\right)=\left[\begin{array}{cccc}2 v_{y} q_{4}-2 v_{z} q_{3} & 2 v_{z} q_{4}+2 v_{y} q_{3} & 2 v_{y} q_{2}-4 v_{x} q_{3}-2 v_{z} q_{1} & 2 v_{z} q_{2}-4 v_{x} q_{4}+2 v_{y} q_{1} \\ 2 v_{z} q_{2}-2 v_{x} q_{4} & 2 v_{z} q_{1}-4 v_{y} q_{2}+2 v_{x} q_{3} & 2 v_{z} q_{4}+2 v_{x} q_{2} & 2 v_{z} q_{3}-4 v_{y} q_{4}-2 v_{x} q_{1} \\ 2 v_{x} q_{3}-2 v_{y} q_{2} & 2 v_{x} q_{4}-4 v_{z} q_{2}-2 v_{y} q_{1} & 2 v_{y} q_{4}-4 v_{z} q_{3}+2 v_{x} q_{1} & 2 v_{y} q_{3}+2 v_{x} q_{2}\end{array}\right]$

Equation 19 and 20 provide the expression of the algorithm in a general form and thus can be used for any known field and its corresponding observation. For the AFCKF, the known field is the acceleration due to gravity, which is having components along only one principle axis of the earth frame. Equation 21 and 22 give the normalised gravity field with an added null dimension and the normalised corrected accelerometer readings with an added null dimension respectively. Substituting equations 21 and 22 in equations 19 and 20 reduces these equations to equations 23 and 24 respectively.

$$
\begin{gathered}
\hat{g}=\left[\begin{array}{llll}
0 & 0 & 0 & 1
\end{array}\right] \\
\hat{a}_{c o r}=\left[\begin{array}{llll}
0 & a_{c o r_{x}} & a_{\text {cor }_{y}} & a_{\text {cor }_{z}}
\end{array}\right] \\
f_{g}(q, \hat{g}, \hat{a})=\left[\begin{array}{lll}
2\left(q_{2} q_{4}-q_{1} q_{3}\right)-a_{c o r_{x}} \\
2\left(q_{2} q_{1}+q_{4} q_{3}\right)-a_{c o r_{y}} \\
2\left(\frac{1}{2}-q_{2}^{2}-q_{3}^{2}\right)-a_{c o r_{z}}
\end{array}\right] \\
J_{g}^{T}(q, \hat{g})=\left[\begin{array}{cccc}
-2 q_{3} & 2 q_{4} & -2 q_{1} & 2 q_{2} \\
2 q_{2} & 2 q_{1} & 2 q_{4} & 2 q_{3} \\
0 & -4 q_{2} & -4 q_{3} & 0
\end{array}\right]
\end{gathered}
$$

Equation 25 provides the attitude estimate at the current time step given the optimum attitude estimate of previous time steps and the current accelerometer reading. $q_{\nabla, t}$ is the attitude estimate of the current time step obtained using the gradient descent algorithm.

$$
\begin{gathered}
q_{\nabla, t}=q_{t-1}-u_{t} \frac{\nabla f_{g}}{\left\|\nabla f_{g}\right\|} \\
\nabla f_{g}=J_{g}^{T}\left(q_{t-1}, \hat{g}\right) f_{g}\left(q_{t-1}, \hat{g}, \hat{a}_{c o r_{t}}\right)
\end{gathered}
$$

The convergence of the optimisation process depends on the value of the step size $\left(\mu_{t}\right)$. A typical optimisation approach needs multiple iterations to obtain a new attitude estimate given a new accelerometer observation. To increase the efficiency of the optimisation process, the step size $\left(\mu_{t}\right)$ needs to be adjusted at every time step. This can be done using the second derivative of the minimisation function called Hessian. However, calculating the Hessian increases the computational load and is not required for the application, and thus a simpler approach is used instead. If the 
convergence rate is equal to or higher than the physical rate of change of the attitude, then only one iteration per sample time is enough to obtain an attitude estimate with sufficient accuracy. Since the convergence rate is governed by $\mu_{t}$, the appropriate value of $\mu_{t}$ can ensure that the convergence of the attitude is limited by the physical rate of change of the attitude, avoiding overshooting. The value of $\mu_{t}$ is given by equation 26 , where $\beta$ is a scaling constant that accounts for accelerometer noise, $\dot{q}_{\omega, t}$ is the current physical rate of change of the attitude (measured by the gyroscope) and $\Delta t$ is the time step.

$$
\mu_{t}=\beta\left|\dot{q}_{\omega, t}\right| \Delta t, \beta>1
$$

This process ensures that the step size is adapted according to the physical rate of change of the attitude. Thus, if the attitude is changing slowly, the step size is smaller, and vice versa.

\subsection{Attitude from angular velocity}

The rate gyroscope measures the quadcopter's angular rate (angular velocity) with respect to the inertial frame represented in the body frame of reference. The angular rate is used to obtain the rate quaternion $(\dot{q})$ using equation 27 (Cooke et al. (1992)), where $\omega_{t}$ is the current time step gyro reading and $q_{t-1}$ is the previous time step optimum attitude estimate.

$$
\dot{q}_{\omega, t}=\frac{1}{2} q_{t-1} \otimes\left[\begin{array}{llll}
0 & \omega_{x_{t}} & \omega_{y_{t}} & \omega_{z_{t}}
\end{array}\right]
$$

Given the previous time step optimum attitude estimate and $\Delta t$, a numerical integration can be used to obtain the current time step attitude. This is shown in equation 28 , where $q_{\omega, t}$ is the attitude estimate obtained from the gyroscope.

$$
q_{\omega, t}=q_{t-1}+\dot{q}_{\omega, t} \Delta t
$$

\subsection{Optimal roll and pitch estimates using the Kalman filter}

As mentioned in the introduction, an extended Kalman filter is used to obtain the optimum roll and pitch estimates of the quadcopter. The Kalman filter consists of a process model and a sensor model. The gyroscope readings are the input into the process model, while the sensor model consists of the attitude estimate obtained from the gradient descent algorithm. Both multiplicative (MEKF) and additive (AEKF) Kalman Filters are widely used for quaternion representation based attitude estimators (Marins et al. (2001), Xiaoping et al. (2005), Liu et al. (2014), Qin et al. (2018)). The MEKF conceptually aligns with the quaternion representation, but the AEKF and MEKF are mathematically equivalent, provided that the AEKF is properly constrained (Crassidis et al. (2007), Lefferts et al. (1982), Shuster (2003a), Shuster (2003b)). For the sake of completion, both an additive and a multiplicative version of the Kalman Filter used in the present estimator are shown in the following sections. 


\subsubsection{AEKF}

In quaternion-based AEKF, all four quaternions are considered to be separate variables. The AEKF utilises summation operations, which does not conceptually align with the quaternion representation of the attitude. However, as mentioned above, it is numerically equivalent to the MEKF if properly constrained, as is done in the present research.

\section{Process model}

The process model of the Kalman filter consists of the gyroscope readings and is given by equations 27 and 28. The gyroscope is used to obtain the angular rate of the quadcopter. However, gyroscope readings are corrupted by noise and bias. The stationary bias $\left(\delta_{\omega}\right)$ is removed from the gyroscope readings through calibration. The gyroscope noise, $e_{\omega}$, is assumed to be Gaussian distributed white noise with zero mean and a known standard deviation given by equation $29\left(I_{3 \times 3}\right.$ is an identity matrix of three dimensions). It is also assumed that there is no correlation between the noise of the three gyroscope axis readings. Furthermore, the gyroscope is plagued with a bias drift. Thus, after the stationary bias correction, the gyroscope measurements consist of real angular velocity $(\omega)$ that is unknown, gyro noise $\left(e_{\omega}\right)$, and the gyro bias rift $\left(b_{\omega_{t}}\right)$ as shown in equation 30 .

$$
\begin{gathered}
\Sigma_{\omega}=\left[\begin{array}{ccc}
\sigma_{\omega_{x}} & \sigma_{\omega_{y}} & \sigma_{\omega_{z}}
\end{array}\right] I_{3 \times 3} \\
\omega_{\text {gyro }_{t}}=\omega_{t}+e_{\omega}+b_{\omega_{t}}
\end{gathered}
$$

The gyroscope readings are corrected for the bias drift using an orientation filter based on the feedback of the error in the rate of attitude change, as is shown in the later section of this paper. Thus, after the bias drift correction and using equation 27,28 , and 30 , the process mode is given by equation 31 .

$$
q_{\omega, t}=q_{t-1}+\frac{1}{2} q_{t-1} \otimes\left(\left[\begin{array}{ll}
0 & \omega_{t}
\end{array}\right]+\left[\begin{array}{ll}
0 & e_{\omega}
\end{array}\right]\right) \Delta t
$$

Where:

$$
\begin{gathered}
\omega_{t}=\left[\begin{array}{lll}
\omega_{x_{t}} & \omega_{y_{t}} & \omega_{z_{t}}
\end{array}\right] \\
e_{\omega}=\left[\begin{array}{lll}
e_{x} & e_{y} & e_{z}
\end{array}\right]
\end{gathered}
$$

Equation 31 can be expressed as equation 32 , where $e_{q, \omega}$ is a zero mean white noise (process model noise). Thus, the final Kalman filter process model and process noise co-variance matrix are given by equation 33 , where $\omega_{c o r, t}$ is the gyroscope reading compensated for bias drift, and $E\left[x x^{T}\right]$ is 
the expectation operator.

$$
q_{\omega, t}=q_{t-1}+\frac{1}{2} q_{t-1} \otimes\left[\begin{array}{ll}
0 & \omega_{t}
\end{array}\right] \Delta t+e_{q, \omega}
$$

Where:

$$
\begin{aligned}
& e_{q, \omega}=\frac{1}{2} q_{t-1} \otimes\left[\begin{array}{ll}
0 & e_{\omega}
\end{array}\right] \Delta t \\
& q_{\omega, t}=q_{t-1}+\frac{1}{2} q_{t-1} \otimes\left[\begin{array}{ll}
0 & \omega_{c o r, t}
\end{array}\right] \Delta t \\
& Q_{t}=E\left[e_{q, \omega} e_{q, \omega}^{T}\right]=\frac{\Delta t^{2}}{4} q_{t-1} \otimes\left[\begin{array}{llll}
0 & \sigma_{\omega_{x}}^{2} & \sigma_{\omega_{y}}^{2} & \sigma_{\omega_{z}}^{2}
\end{array}\right] I_{4 \times 4} \otimes q_{t-1}^{T}
\end{aligned}
$$

\section{Sensor model}

The sensor model consists of the attitude estimate obtained via the gradient descent algorithm, as well as the measurement model noise, and is given by equation 34 . The measurement noise covariance matrix is given by equation 35 , where $\mu_{t}$ is the step size of the gradient descent algorithm. $J_{g}$ is given by equation $24, \nabla f_{g}$ is given by equation 25 , and $\Sigma_{a c c}$ is the known noise characteristics of the accelerometer, given by equation 36. It is assumed that there is no correlation between the noise of the different axes of the accelerometer.

$$
\begin{gathered}
q_{\nabla, t}=I_{4 \times 4} q_{\nabla, t} \\
R_{t}=\frac{\mu_{t}^{2}}{\left\|\nabla f_{g}\right\|^{2}} J_{g}^{T} \Sigma_{a c c}^{2} J_{g} \\
\Sigma_{a c c}=\left[\begin{array}{lll}
\sigma_{a c c_{x}} & \sigma_{a c c_{y}} & \sigma_{a c c_{z}}
\end{array}\right] I_{3 \times 3}
\end{gathered}
$$

\section{Derivation of the sensor model}

Similar to the process noise co-variance matrix, the measurement noise co-variance matrix is also given by the expectation of the measurement model noise $\left(E\left[e_{q, a}, e_{q, a}\right]\right)$. Equation 25 can be written as equation 37 ,

$$
q_{\nabla, t}=q_{t-1}-\Upsilon\left[\begin{array}{l}
2\left(q_{2} q_{4}-q_{1} q_{3}\right)-a_{c o r_{x}} \\
2\left(q_{2} q_{1}+q_{4} q_{3}\right)-a_{c o r_{y}} \\
2\left(\frac{1}{2}-q_{2}^{2}-q_{3}^{2}\right)-a_{c o r z}
\end{array}\right]
$$

where for every time-step, $\Upsilon$ is a deterministic constant matrix and is given by equation 38 . 


$$
\Upsilon=u_{t} \frac{J_{g}^{T}\left(q_{t-1}, \hat{g}\right)}{\left\|\nabla f_{g}\right\|}
$$

The corrected accelerator reading consists of unknown real quadcopter acceleration and noise (zero mean white Gaussian noise with a known standard deviation), as given by equation 39 .

$$
\vec{a}_{\text {cor }}=\vec{a}_{\text {real }}+e_{a}
$$

Using equations 37 and 39 , equation 40 can be obtained.

$$
q_{\nabla, t}=q_{t-1}-\Upsilon\left[\begin{array}{l}
2\left(q_{2} q_{4}-q_{1} q_{3}\right)-a_{x} \\
2\left(q_{2} q_{1}+q_{4} q_{3}\right)-a_{y} \\
2\left(\frac{1}{2}-q_{2}^{2}-q_{3}^{2}\right)-a_{z}
\end{array}\right]-\Upsilon\left[\begin{array}{c}
e_{a_{x}} \\
e_{a_{y}} \\
e_{a_{z}}
\end{array}\right]
$$

Since the output of equation 40 is the Kalman filter measurement model, the measurement model noise is given by equation 41 .

$$
e_{q, a}=\Upsilon\left[\begin{array}{l}
e_{a_{x}} \\
e_{a_{y}} \\
e_{a_{z}}
\end{array}\right]
$$

The measurement model noise co-variance matrix that is equal to $E\left[e_{q, a} e_{q, a}^{T}\right]$ is given by equation 42 .

$$
E\left[e_{q, a}, q_{q, a}^{T}\right]=\Upsilon E\left[e_{a} e_{a}^{T}\right] \Upsilon^{T}=\Upsilon \Sigma_{a c c}^{2} \Upsilon^{T}=\frac{\mu_{t}^{2}}{\left\|\nabla f_{g}\right\|^{2}} J_{g}^{T} \Sigma_{a c c}^{2} J_{g}
$$

\section{AEKF Algorithm}

Given the previous time-step's optimum attitude estimate and co-variance matrix, the following steps are followed to obtain the current time step's attitude estimate. The Kalman filter is initiated with the initial estimate $\left(q_{0}\right)$ being the true initial attitude and the state estimation error covariance matrix $\left(P_{0,0}\right)$ as a large value matrix $\left(P_{0,0}=\infty\right)$. The matrices $\Phi, \Lambda$ and $H$ are identity matrices of four dimensions. 


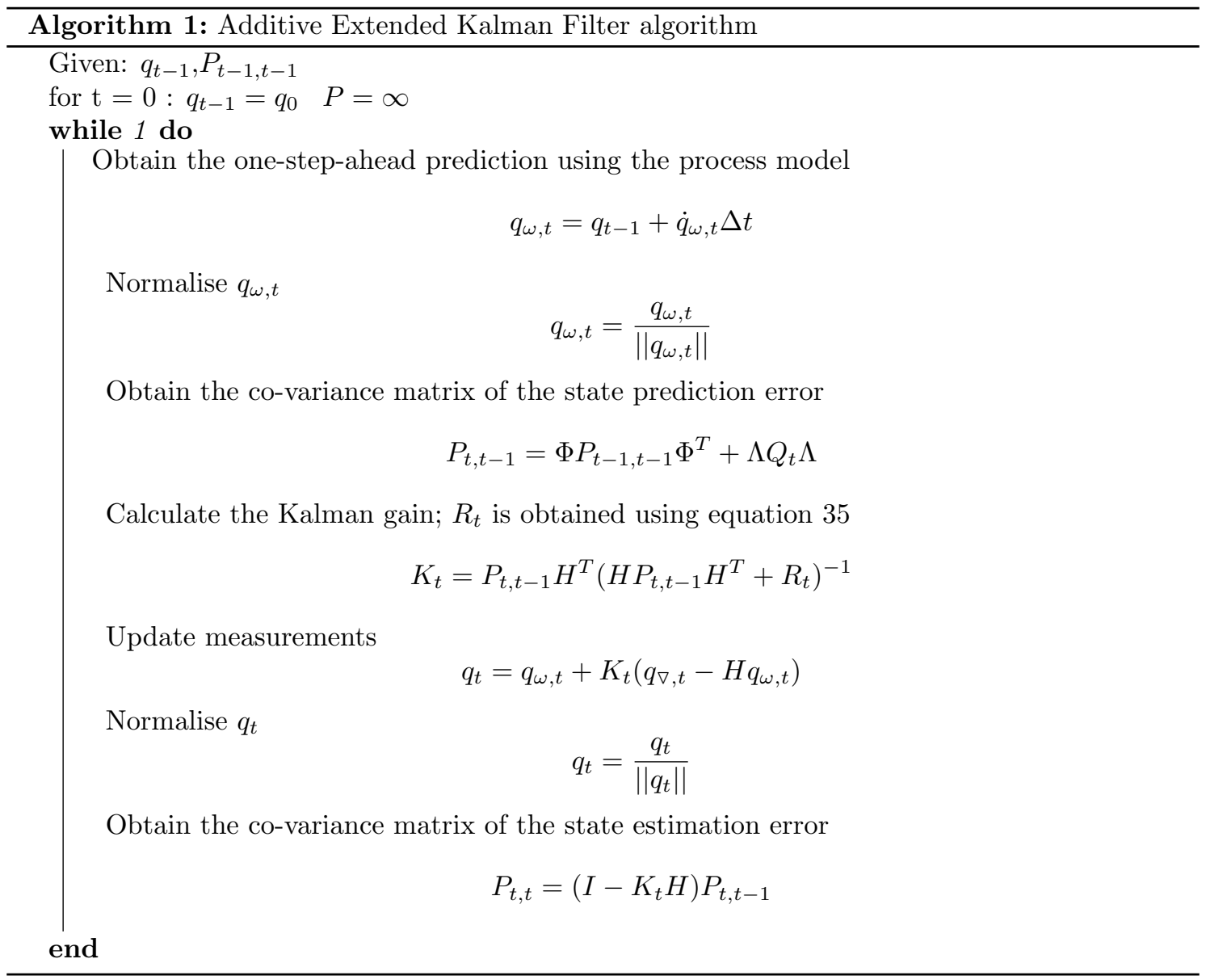

Although the centrifugal model is meant to remove some of the external acceleration from the accelerometer readings, the quadcopter can still be affected by some linear acceleration. The effects of such unanticipated acceleration are reduced by adapting the measurement noise covariance matrix $\left(R_{t}\right)$. The difference between the norm of the corrected accelerometer $\left(\vec{a}_{c o r}\right)$ reading and the norm of the normalised gravity vector (unity) is obtained and then used to alter the measurement noise co-variance matrix, as shown in equation 43 , where $\xi$ is given by equation 44 . Adapting the measurement model noise co-variance matrix in such a manner reduces the impact of a sudden linear acceleration on the attitude estimates, because an increase in the measurement model noise co-variance matrix shifts the Kalman gain to rely stronger on the measurement model estimates, i.e., the attitude estimates obtained via the rate gyroscope.

$$
\begin{gathered}
\left\{\begin{array}{c}
R_{t}=\infty \text { if }|\xi|>\lambda_{a} \\
R_{t}=R_{y} \text { if }|\xi|<\lambda_{a}
\end{array}\right\} \\
\xi=\left\|\vec{a}_{c o r}\right\|-1
\end{gathered}
$$

The value of $\lambda_{a}$ depends on the value of the known standard deviation of the accelerometer, i.e., $\lambda_{a}$ 
depends on the noise of the accelerometer. Given the standard deviation of Gaussian distribution (or any distribution), it is possible to tell how far the measurements can spread out from the mean. Thus, if no external acceleration (external noise) is present, i.e., the accelerometer readings consist of only the gravity vector and random white noise, and the standard deviation of the accelerometer is known, then the spread of the accelerometer readings is given by equation 45 , where $\vec{a}_{\text {spread }_{I}}$ is the spread of the accelerometer readings in the earth frame. Equation 45 is only valid under the assumption that the noise in the accelerometer readings along each principle axis are uncorrelated, i.e., the standard deviation of the accelerometer readings is given by equation 12 .

$$
\vec{a}_{\text {spread }}=\frac{\vec{g}}{\|\vec{g}\|} \pm \alpha\left[\begin{array}{lll}
1 & 1 & 1
\end{array}\right] \Sigma_{a}, \alpha=1,2, \ldots n
$$

Thus, if the spread of the accelerometer readings in the non-accelerated scenario is known, the maximum deviation of the modulus of the accelerometer readings can be obtained using equation 46 , where $\lambda_{a}$ is the modulus of the spread of the accelerometer readings.

$$
\lambda_{a}=\left\|\frac{\vec{g}}{\|\vec{g}\|}+\alpha\left[\begin{array}{lll}
1 & 1 & 1
\end{array}\right] \Sigma_{a}\right\|, \alpha=1,2, \ldots n
$$

Furthermore, if the value of alpha is chosen to be equal to 3, then, assuming a Gaussian distribution of the noise, $\lambda_{a}$ includes $99.9 \%$ of the accelerometer readings. If the $\xi$ exceeds $\lambda$, it implies that the corrected accelerometer readings are corrupted with external acceleration. Thus, the measurement noise co-variance matrix is adjusted accordingly. The quaternion estimates obtained from the Kalman filter are the optimum attitude estimates of the roll $(\phi)$ and pitch $(\theta)$ attitude of the quadcopter. The obtained yaw $(\psi)$ estimates cannot be trusted, as only gyroscope readings and the gravity vector are used for the attitude estimate, and the gravity vector runs parallel to the yaw axis of the quadcopter in the earth frame.

\subsubsection{MEKF}

Other than the AEKF, the MEKF conceptually aligns with the quaternion representation, because it adheres to the dimensionality of the rotation group. Furthermore, its attitude estimate is defined as being a unit quaternion (Markley (2004)).

\section{Process model}

As quaternion addition and subtraction are not defined, the MEKF utilises the quaternion multiplicative error as a state Liu et al. (2014). Using equation 5, the error quaternion is given by equation 47 .

$$
\delta q=q \otimes \hat{q}^{*}
$$


The MEKF is based on the assumption that the attitude of the system/quadcopter only changes by a minimal amount $((\delta \phi, \delta \theta, \delta \psi)<<1)$ in every time step (Liu et al. (2014), Burton et al. (2013)). Using this assumption, the error quaternion can be written as shown in equation 48 .

$$
\delta q=\delta q(a) \approx\left[\begin{array}{ll}
1 & a
\end{array}\right]^{T}
$$

Where $a$ is a three-parameter vector, i.e., $a \in \mathbb{R}^{3}$. Furthermore, $a$ is approximately equal to half of the change in the Euler angles between two quaternions (under the assumption that Euler angles $((\phi, \theta, \psi)<<1)$, as shown by equation 49 (Jiang et al. (2016)).

$$
a \approx \frac{1}{2}\left[\begin{array}{lll}
\phi & \theta & \psi
\end{array}\right]^{T}
$$

Differentiating equation 49 leads to equation 50.

$$
\dot{a} \approx \frac{1}{2}\left[\begin{array}{lll}
\dot{\phi} & \dot{\theta} & \dot{\psi}
\end{array}\right]^{T}
$$

The kinematic model of the quadcopter is given by equation 27 . Using equation 50 and 27 and the differentiation of equation 47 , the process model in terms of the error quaternion is given by equation 51 (Battiston et al. (2017)).

$$
\delta \dot{q}=\left[\begin{array}{ll}
0 & \delta \dot{\vec{q}}
\end{array}\right]^{T}=\left[\begin{array}{ll}
0 & -[\omega \times] \delta \vec{q}+I_{3 \times 3} e_{\omega}
\end{array}\right]^{T}
$$

where $\delta \vec{q}$ is the vector part of the quaternion error, and $[\omega \times]$ is given by equation 52 .

$$
[\omega \times]=\left[\begin{array}{ccc}
0 & -\omega_{3} & \omega_{2} \\
\omega_{3} & 0 & -\omega_{1} \\
-\omega_{2} & \omega_{1} & 0
\end{array}\right]
$$

In the MEKF, the scalar part of the quaternion is neglected because it does not change due to the small angle assumption (Liu et al. (2014), Markley (2004)). Thus, the final process model and the process model noise co-variance matrix $(Q)$ are given by equations 53 and 54 respectively.

$$
\begin{gathered}
\delta \dot{\vec{q}}=-[\omega \times] \delta \vec{q}+I_{3 \times 3} e_{\omega}=-\left[\omega_{c o r} \times\right] \delta \vec{q} \\
Q=\Sigma_{\omega} \Sigma_{\omega}^{T}=\left[\begin{array}{lll}
\sigma_{\omega_{x}}^{2} & \sigma_{\omega_{y}}^{2} & \sigma_{\omega_{z}}^{2}
\end{array}\right] I_{3 \times 3}
\end{gathered}
$$


Furthermore, equation 53 can be written as equation 55.

$$
\delta \dot{\vec{q}}=-[\omega \times] \delta \vec{q}+I_{3 \times 3} e_{\omega}=F \delta \vec{q}+G e_{\omega}
$$

\section{Sensor model}

The MEKF sensor model is same as the sensor model of the AEKF. Since only the error quaternion is used and the change in the scalar part of the quaternion is neglected, the sensor model noise co-variance matrix is adjusted by removing the respective row and column, i.e., $R_{t}$ of the MEKF is given by removing the first row and column from the noise co-variance matrix obtained from equation 35. Matrix $H$ is a three dimension identity matrix.

\section{MEKF Algorithm}

Setting the initial attitude estimates $\left(q_{0}\right)$ as true estimates and the initial estimate error co-variance matrix $\left(P_{0,0}\right)$ as a large value matrix $\left(P_{0,0}=\infty\right)$, the MEKF algorithm is given by algorithm 2 .

\subsection{Gyroscope bias drift correction}

Due to temperature changes and the motion of the quadcopter, the gyroscope's bias changes over time. One way to correct for the drift in bias is to estimate the bias using a Kalman filter (Foxlin (1996), Jurman et al. (2007), Gebre-Egziabher et al. (2004), Sabatini (2006)), but doing so leads to an increase in the number of states of the Kalman filter, which directly affects the computational cost of the overall filter. However, another filter developed by Mahony et al. (2008) utilises the integrated feedback of error between the current accelerometer reading and the projection of the gravity vector in the sensor frame to correct for the gyroscopic bias drift. The gravity vector is projected using the last time step's optimum attitude estimate. An approach based on Mahony et al. (2008) will be utilised to correct for the gyroscope bias drift in the AFCKF. The compensated gyroscope readings are given by equation 56 , where $\omega_{\text {gyro,t }}$ is the current gyroscope reading.

$$
\begin{aligned}
\omega_{c o r, t} & =\omega_{g y r o, t}-b_{\omega, t} \\
b_{\omega, t} & =\rho \sum_{t} \omega_{e, t} \Delta t
\end{aligned}
$$

where:

$$
\omega_{e, t}=\vec{a}_{c o r} \times\left(q_{t-1} \otimes \frac{\vec{g}_{I}}{\left\|\vec{g}_{I}\right\|} \otimes q_{t-1}\right)
$$

$\rho$ is the integral gain and $\omega_{e, t}$ is the error between the corrected accelerometer readings and the projection of the gravity vector (inertial frame) onto the body frame, which is made using the 


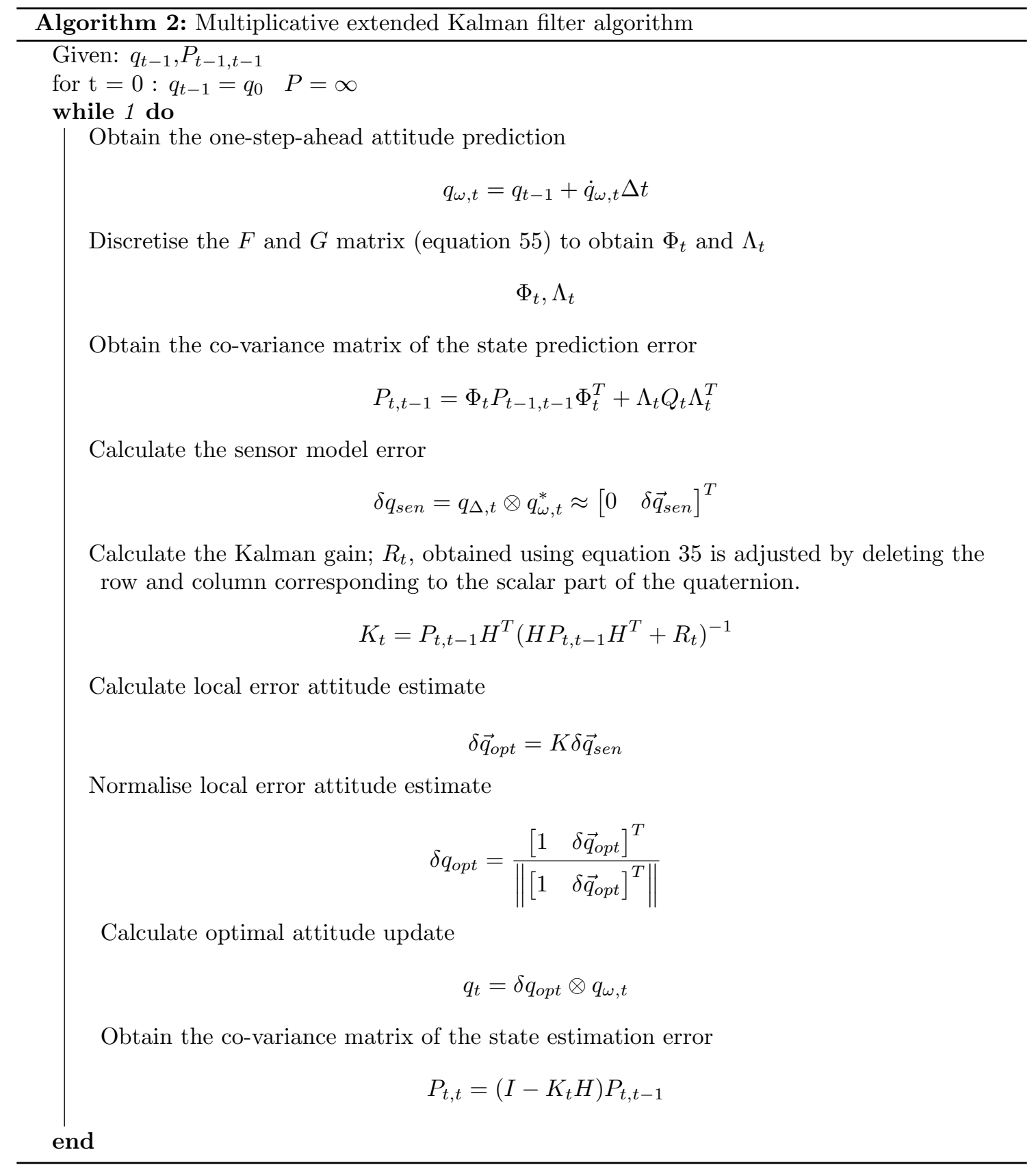


last time step's attitude estimates. The integral gain $\rho$ depends on the estimated rate of change of the gyroscope bias drift. The integral constant value can be obtained in a manner similar to Madgwick et al. (2011). Equation 58 provides the value of the integral constant $\rho$, where $\hat{q}$ is any unit quaternion and $\omega_{d}$ is the estimated rate of change of the gyroscopic bias drift. If the estimated rate of change of the gyroscope bias drift is assumed to be the same along each axis of the gyroscope, equation 58 can be simplified as equation 59 , where $\omega_{d}$ is the same along each axis of the gyroscope.

$$
\begin{gathered}
\rho=\left\|\frac{1}{2} \hat{q} \otimes\left[\begin{array}{llll}
0 & \omega_{d_{x}} & \omega_{d_{y}} & \omega_{d_{z}}
\end{array}\right]\right\| \\
\rho=\sqrt{\frac{3}{4} \omega_{d}}
\end{gathered}
$$

\subsection{Yaw estimate}

The attitude estimator is designed in such a way that the yaw $(\psi)$ estimate is obtained via a channel separate from the roll and pitch estimates of the quadcopter. This approach has a significant advantage over the approach in which the magnetometer readings are incorporated in obtaining the complete attitude. Since the magnetometer is only used for obtaining the yaw estimate, external magnetic disturbance affects only this estimateand not he roll and pitch estimates, which are more crucial for the stability of the quadcopter.

To obtain the yaw estimate, the yaw rate measured by the gyroscope is integrated and added to the yaw estimate obtained via the magnetometer reading through a linear gain. The linear gain is decided using fuzzy logic to cater for magnetic disturbance. The yaw estimate obtained from the gyroscope reading via Newton integration is shown in equation 60 .

$$
\psi_{\omega, t}=\psi_{t-1}+\dot{\psi}_{t} \Delta t
$$

Another set of yaw estimates is obtained from the magnetometer. The magnetometer readings need to be compensated for the roll and pitch of the quadcopter in order to be used for yaw estimation. Eclipse hypothesis compensation (Tao et al. (2013)) is used to compensate for the roll and pitch of the quadcopter as given by equation 61 .

$$
\left[\begin{array}{l}
m_{\text {com }_{x}} \\
m_{c o m_{y}} \\
m_{\operatorname{com}_{z}}
\end{array}\right]^{T}=\left[\begin{array}{c}
m_{x} \cos \left(\phi_{t}\right)-m_{z} \sin \left(\phi_{t}\right) \\
m_{x} \sin \left(\phi_{t}\right) \sin \left(\theta_{t}\right)+m_{y} \cos \left(\theta_{t}\right)+m_{z} \cos \left(\psi_{t}\right) \sin \left(\theta_{t}\right) \\
m_{x} \sin \left(\phi_{t}\right) \cos \left(\theta_{t}\right)-m_{y} \sin \left(\theta_{t}\right)+m_{z} \cos \left(\phi_{t}\right) \cos \left(\theta_{t}\right)
\end{array}\right]^{T}
$$

The compensated magnetometer reading is used to calculate the yaw angle using equation 62 .

$$
\psi_{m, t}=\operatorname{acrtan}\left(\frac{m_{\text {com }_{y}}}{m_{\text {com }_{y}}}\right)+\nu
$$


where $\nu$ is the angle between the geographical north and magnetic north for the given geographical location. The final yaw estimate, which is a weighted sum of the yaw estimate obtained via the magnetometer and the yaw estimate obtained via the rate gyroscope, is obtained using a fuzzy complementary filter, as shown in equation 63 .

$$
\psi_{t}=(1-\kappa) \psi_{\omega, t}+\kappa \psi_{m, t}
$$

$\kappa$ is the filter gain that is obtained using fuzzy logic. The gain of the filter is based on fuzzy logic to cater for the external magnetic field in the environment and the saturation of the gyroscope, since the gyro readings cannot be trusted once the gyroscope is saturated. The accuracy of the weighted summation, i.e., the optimum yaw estimate, depends on the choice of the weight (gain), which needs to be carefully tuned. If a constant gain was used, it would lead to sub-optimal yaw estimates and would not be able to accommodate external magnetic field disturbances and rate gyroscope saturation.

Therefore, a fuzzy gain interpolation scheme is used, such that the gain is altered based on two criteria: Firstly, the error between the norm of the magnetic field in the inertial frame and the norm of the magnetometer readings, and secondly, the modulus of difference between the gyroscope yaw saturation point and the current gyroscope readings. The fuzzy gain calculation consists of three steps: fuzzification of the crisp input, inference based on the rules, and defuzzification to return a crisp value back (Cox (1992)). Fuzzification is based on two variables (also called fuzzifying variables): firstly, the modulus error between the norm of the local earth's magnetic field and the norm of the measured magnetometer reading, given by $\varrho_{|m|}$, and secondly, the modulus of the yaw rate measured by the gyroscope, given by $\left|\omega_{\psi}\right|$. The membership functions used for fuzzifying $\varrho_{|m|}$ and $\omega_{\psi}$ are given by equations 64 and 65 respectively.

$$
\begin{gathered}
y\left(\varrho_{|m|}\right)=\frac{e^{\frac{\ln \left(\vartheta_{m}\right)}{\lambda_{m}} \varrho_{|m|}}-1}{\vartheta_{m}-1} \\
y^{\prime}\left(\varrho_{|m|}\right)=1-y\left(\varrho_{|m|}\right) \\
y\left(\omega_{\psi}\right)=\frac{e^{\frac{\ln \left(\vartheta_{\omega}\right)}{\omega_{\psi}, s a t}\left|\omega_{\psi}\right|}-1}{\vartheta_{\omega}-1} \\
y^{\prime}\left(\omega_{\psi}\right)=1-y\left(\omega_{\psi}\right)
\end{gathered}
$$

The membership functions' graphs are shown in figure 3.2. 


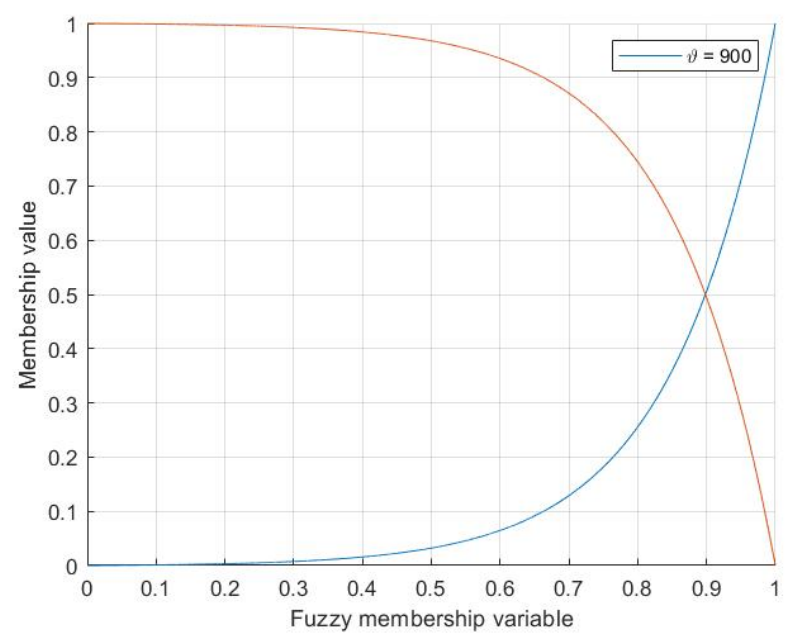

Figure 3.2: Fuzzifying membership function

It can be observed in equations 64 and 65 that the curvature of the membership function depends on the value of $\vartheta\left(\vartheta_{\omega}\right.$ or $\left.\vartheta_{m}\right)$, as shown in figure 3.3. For a higher value of $\vartheta$, the respective membership function curves more radically towards the higher value of the respective fuzzifying variable. $\vartheta_{|m|}$ needs to be tuned depending upon the noise level of the magnetometer. Thus $\vartheta_{|m|}$ is chosen such that the curvature of the function is towards the higher error. Similarly, $\vartheta_{\omega}$ is also tuned in a similar manner.

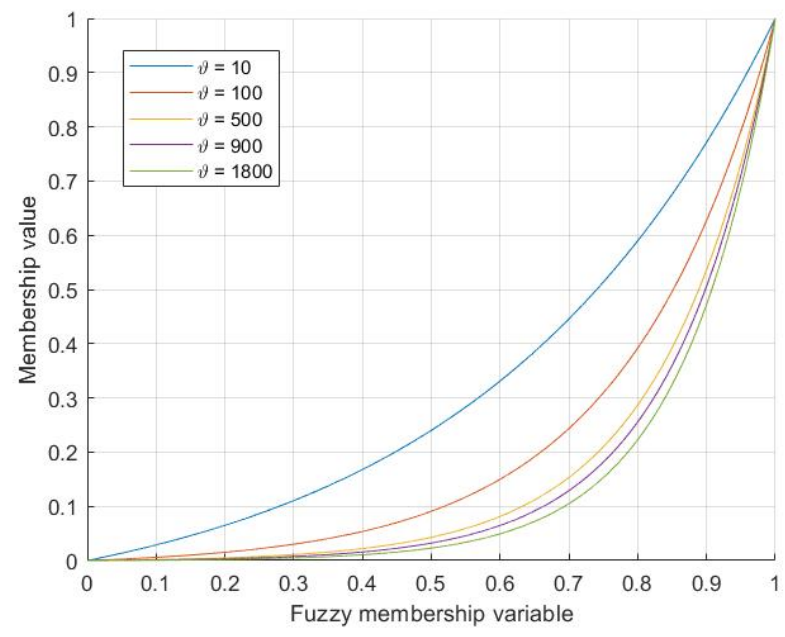

Figure 3.3: Function shape based on different values of $\vartheta$

In equations 64 and $65, \omega_{\psi, s a t}$ is the gyro yaw rate saturation, i.e., the maximum yaw rate the on-board rate gyro can measure, $\omega_{\psi}$ is the current measured yaw rate, and $\lambda_{m}$ is the maximum acceptable error between the norm of the local magnetic field and the norm of the magnetic field measured by the magnetometer. The value of $\lambda_{m}$ depends on the standard deviation of the 
magnetometer and is given by equation 66 .

$$
\lambda_{m}=\alpha\left\|\left[\begin{array}{lll}
1 & 1 & 1
\end{array}\right] \Sigma_{m}\right\|, \alpha=1,2, \ldots n
$$

The value of the maximum acceptable error $\left(\lambda_{m}\right)$ is obtained based on a similar reasoning as the reasoning used to obtain $\lambda_{a}$, as given in equation 46. The error between the norm of the local earth's magnetic field and the norm of the measured magnetometer reading $\left(\varrho_{|m|}\right)$ is given by equation 67 . This implies that if $\varrho_{|m|}$ is greater than the maximum acceptable error, it is set to be equal to the maximum acceptable error.

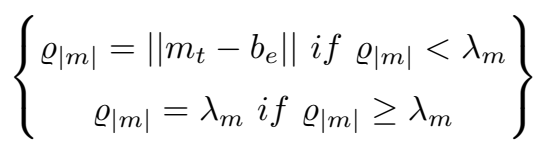

Once the membership values are obtained based on equations 64 and 65 (four membership values are obtained, two for each fuzzyfying variable), then the minimum weight method, also called fuzzy intersection, is used for inference (Beg and Ashraf (2009)). For implementing the inference step, ideal gain values $\left(\kappa_{i}\right)$ for the four extreme case sets needs to be known. This means that for implementing the fuzzy inference steps, we need to know the ideal gains for the combinations of all the extreme cases. For this filter, four gain values are required for the following four cases:

1. Zero magnetometer $\left(y\left(\varrho_{|m|}\right)\right)$ error and no gyroscope saturation $\left(y\left(\omega_{\psi}\right)\right)$

2. Zero magnetometer error $\left(y\left(\varrho_{|m|}\right)\right)$ and gyroscope saturation $\left(y^{\prime}\left(\omega_{\psi}\right)\right)$

3. High magnetometer error $\left(y^{\prime}\left(\varrho_{|m|}\right)\right)$ and no gyroscope saturation $\left(y\left(\omega_{\psi}\right)\right)$

4. High magnetometer $\left(y^{\prime}\left(\varrho_{|m|}\right)\right)$ error and gyroscope saturation $\left(y^{\prime}\left(\omega_{\psi}\right)\right)$

For case one, the gain value is decided by tuning and depends upon the noise of the gyroscope and magnetometer. For case two, since the gyroscope is saturated, its readings cannot be trusted. Thus, it is obvious that the gain needs to be chosen to be close or equal to unity so that the fuzzy complementary filter relies more on the yaw estimates obtained from the magnetometer. For case three, since the magnetometer noise is high, the gain is chosen in such a way that the yaw estimates obtained from the magnetometer readings are not given much weight, i.e., the gain is close to zero. The gain should not be equal to zero because some input is required to correct for errors due to gyroscopic drift. Case four is problematic because no choice of gain can provide reliable yaw estimates.

Each of the above cases has two membership values. For each case, the minimum membership value is chosen and multiplied with the respective gain $\kappa_{i}$. The product is further used for the defuzzification with the center of gravity method, which provides the final gain for the linear complementary filter as shown in equation 68. There are different defuzzification methods (Madau and Feldkamp (1996), Eisele et al. (1994), Leekwijck and Kerre (1999)), but the center 
of gravity/centroid method is chosen because it has a low computational cost and is simple to implement.

$$
\kappa=\frac{\sum y_{i} \kappa_{i}}{\sum y_{i}}
$$

Finally, to cater for the noise in the estimation, an adaptive window moving average scheme is adopted. The moving average is given by equation 69 .

$$
\psi=\frac{\sum_{i} \psi_{i}}{i}, i=1,2,3 \ldots n
$$

An exponential function is used to calculate the window size $i$ as shown in equation 70 . The window size depends on the yaw rate $(\dot{\psi})$, therefore, as the yaw rate increases the window size decreases. Furthermore, $\iota$ is a scaling constant that depends on the sensor frequency, as shown by equation 71 , where $\eta$ is a positive constant that needs to be tuned based on the sensor noise level.

$$
\begin{gathered}
i=\iota e^{-|\dot{\psi}|} \\
\iota=\eta \min \left(\text { freq }_{\omega}, f r e q_{m}\right) \\
\eta>0
\end{gathered}
$$

\section{Experiments, results, and discussion}

After the estimator is derived as described in chapter three, it undergoes a twofold validation: firstly through a high-fidelity simulation developed by Sun et al. (2018a), and secondly through tests with real-time flight data. In the first part of this chapter, the experimental set-up for these tests will be described. After that, the results will be shown and discussed. The flight tests were divided into four cases: Hover flight with and without a damaged rotor, as well as aggressive motion flight with and without a damaged rotor. The estimates obtained by the AFCKF are discussed and compared to three mainstream filters: the filter developed by Madgwick et al. (2011) (for the sake of convenience called 'Madgwick Filter'), the filter developed by Valenti et al. (2015) (for the sake of convenience called 'Complementary Filter'), and the filter developed by Roetenberg et al. (2005) (for the sake of convenience called 'Kalman Filter'). The Madgwick filter is one of the most widely used attitude filters due to its fast execution time, resulting in a low computational cost and its high accuracy, and thus serves as a benchmark for comparison. The other two filters are used as the main Kalman and Complementary filters respectively in Matlab's sensor fusion and tracking toolbox. The estimates obtained from all filters are also compared to the real estimates, i.e., those obtained from the OptiTrack system. In the last part of the chapter, it is shown that 
the additive and multiplicative versions of the AFCKF yield similar results.

\subsection{Experimental set-up}

The filter was tested using data obtained from the flight of a Parrot Bepop 2 drone, which is equipped with a tri-axis accelerometer, magnetometer and rate gyroscope. The sensor measurements provided by the on-board MARG sensor were calibrated and later post-processed. The logged data were then processed through the AFCKF and the other three filters to obtain the attitude estimates of the quadcopter. Since the attitude estimates are obtained using the same sensor data, these estimates from different filters could be compared among each other and used to evaluate the respective filters's performances.

The attitude estimates obtained from the different filters are compared against another set of attitude estimates which is obtained from the Cyber Zoo at TU Delft. The Cyber Zoo is fitted with an OptiTrack motion capture system that consists of twelve high resolution and high frame rate cameras. Using the OptiTrack system, it is possible to obtain the real-time attitude estimates of the quadcopter to an accuracy of $0.1 \mathrm{~mm}$. Thus the attitude estimates obtained from the OptiTrack system are considered to be true estimates of the quadcopter. The drawback of conducting flights inside the Cyber Zoo are unwanted and undetermined magnetic field disturbances. Since real attitude estimates (obtained using the OptiTrack system) are necessary for the attitude estimator validation and there is no OptiTrack system available in the outside environment, it is unavoidable to have indoor flights.

Four different types of flight tests were conducted: Hover flight with and without a damaged single rotor and aggressive motion flight with and without a damaged single rotor. The MARG sensor data, i.e., the magnetometer readings, accelerometer readings and rate gyro readings, were logged. The logged data were then fed to the four different attitude estimators. The four respective attitude estimates obtained were compared against the real attitude of the quadcopter obtained via OptiTrack. Apart from that, the average execution times of all the estimators were noted, which can be used to estimate their relative computational costs. Since all attitude estimators were run on the same device, the relative execution times can be compared with each other. The software used for the comparison was Matlab2020a and the hardware a Dell G7 157588 laptop.

\subsection{Results and Discussion}

The attitude estimator performance is quantified by calculating the RMS (root mean square) of the error between the attitude estimates obtained via the OptiTrack system and the attitude estimates obtained using the attitude estimator. This is a common practice (e.g. Sabatini (2006), Jurman et al. (2007), Xing et al. (2016), Shan et al. (2017)) that is utilised to quantify the performance of an attitude estimator. Furthermore, Euler representation is used to represent the roll $(\phi)$, pitch $(\theta)$ and yaw $(\psi)$ of the quadcopter, since Euler representation is intuitive for understanding the 
motion of the quadcopter. At the same time, Euler representation is subjected to large errors due to the wrapping of the angles, which do however not affect the controller's performance because they only occur because of the representation scheme. For example, if the angles are wrapped from $180^{\circ}$ to $-180^{\circ}$, then small errors at the boundary (at $180^{\circ}$ or $-180^{\circ}$ ) will be represented as very high errors. Since all the filters are based on quaternion representation, the Euler angles are computed using quaternion attitude estimates and equation 9. The results in this section are obtained using the additive version of the AFCKF. As explained previously and shown in a later section, there is no numerical difference between the additive and the multiplicative versions of the Kalman Filter, which is why the additive and multiplicative versions of the AFCKF lead to similar results.

\subsubsection{Hover flight with damaged rotor}

\section{Flight description}

In the first flight test, the quadcopter was made to hover at a point without giving any linear motion commands. Figure 4.1 shows the linear motion of the quadcopter along with the Euler angles of the quadcopter measured using the OptiTrack system. Because of the damaged rotor, the quadcopter rotates about the yaw axis with a high rotational rate of about $15.5 \mathrm{rad} / \mathrm{sec}$. It can be observed that its roll and pitch are maintained at near zero. Furthermore, very little linear motion is observed. 

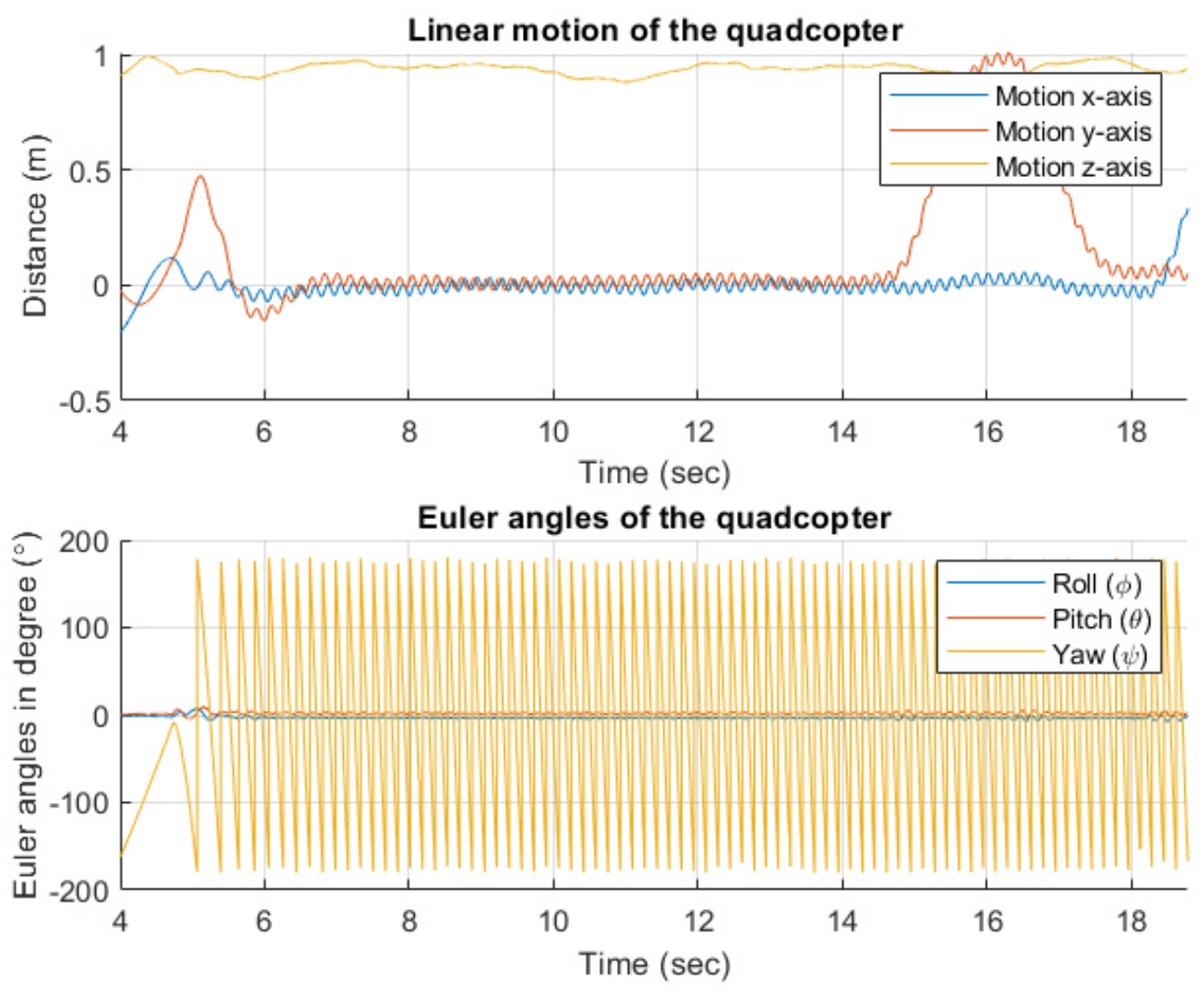

Figure 4.1: Flight path: Hover with damaged rotor

\section{Performance of the estimators}

The MARG sensor data obtained from the flight test were fed to the respective estimators to obtain the quadcopter's attitude estimates. Figures 4.2, 4.3, and 4.4 show the yaw, pitch, and roll estimation error of the different filters respectively. 


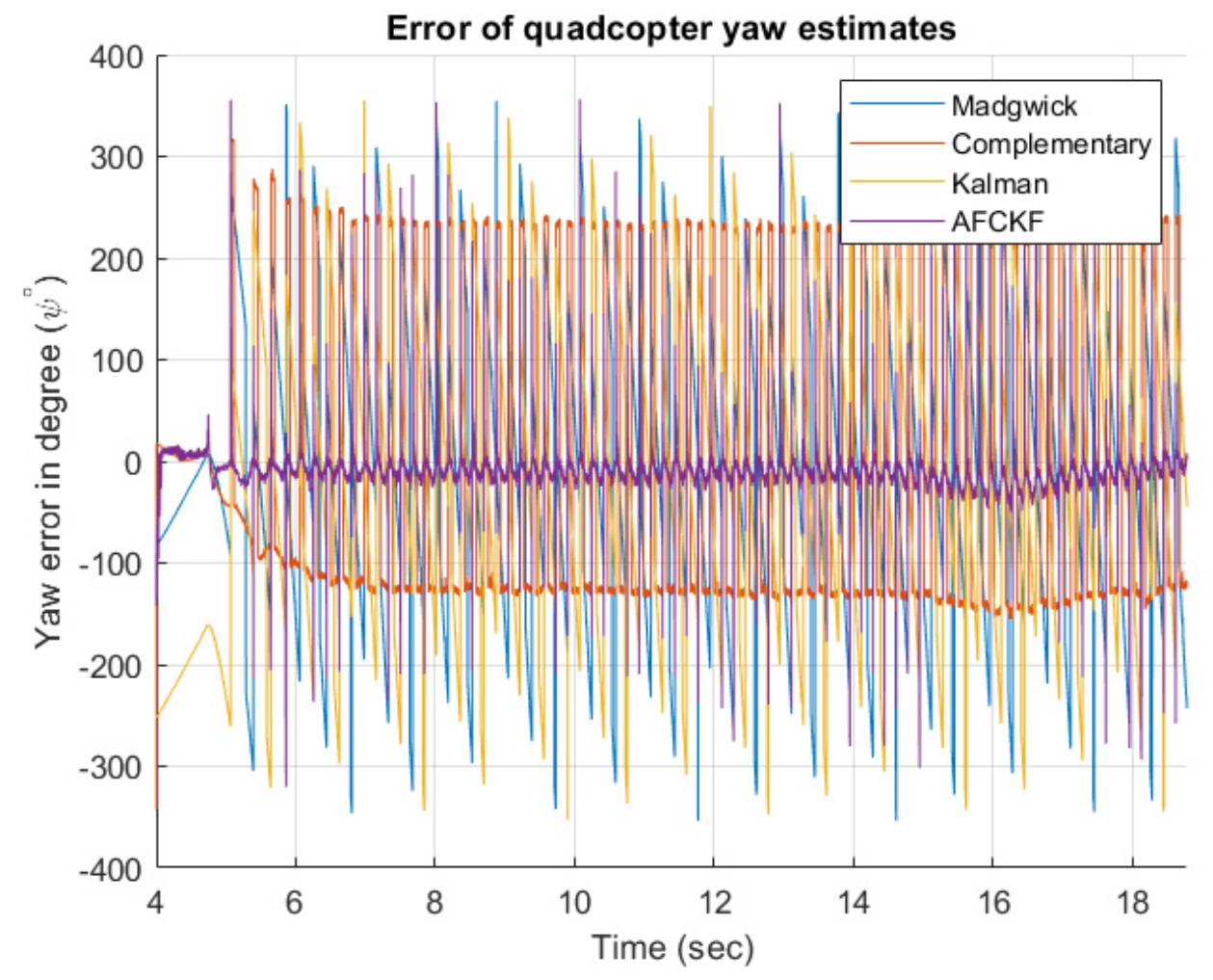

Figure 4.2: Yaw estimate error of different filters [Case: Hover with damaged rotor]

As can be seen in figure 4.2 , in the case of a damaged rotor flight with a high yaw rate, only the AFCKF is able to track the quadcopter's yaw. Although the error stays low in general, sudden peaks can be observed, which occur due to the wrapping of the Euler angles from $-180^{\circ}$ to $180^{\circ}$. 


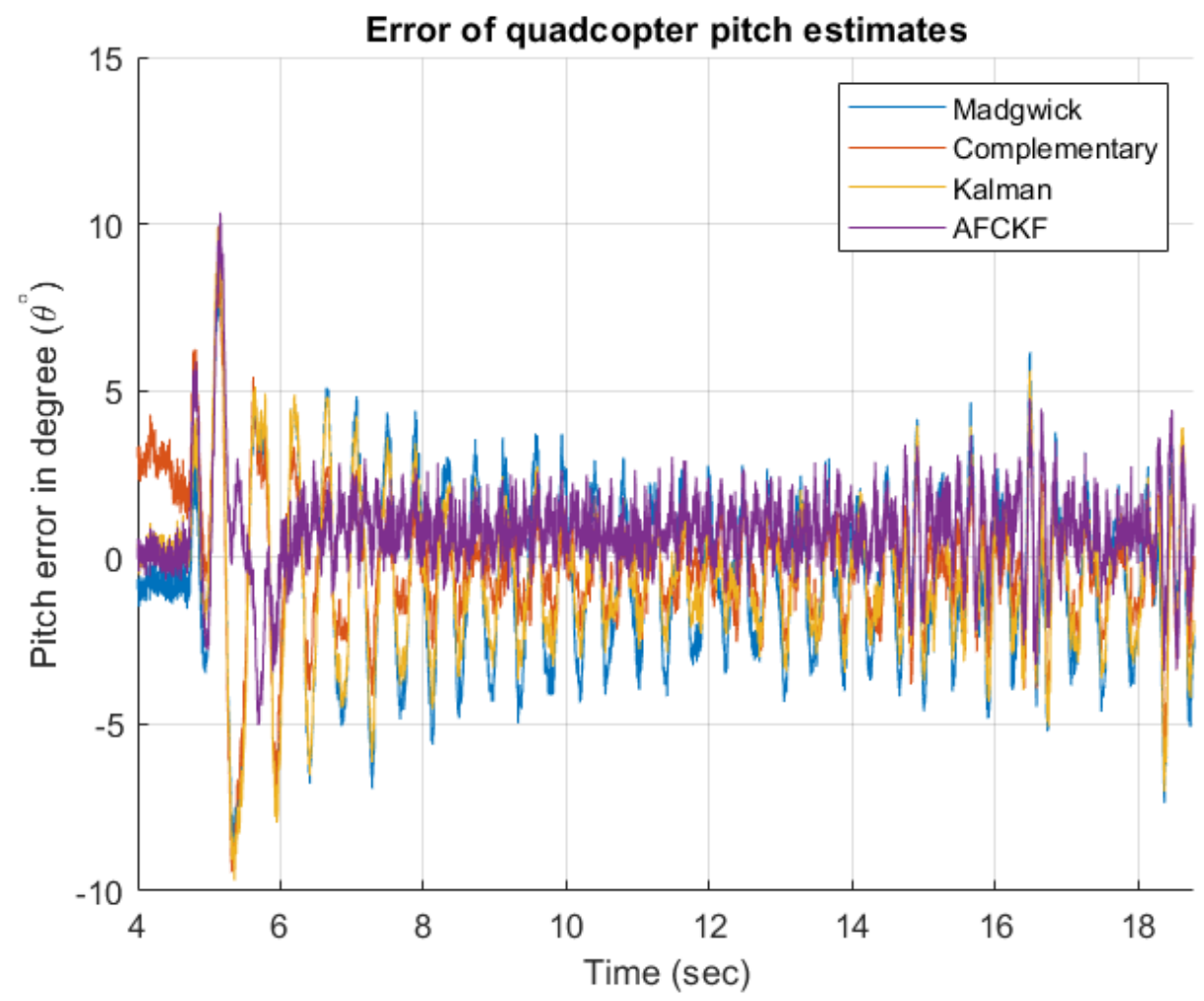

Figure 4.3: Pitch estimate error of different filters [Case: Hover with damaged rotor]

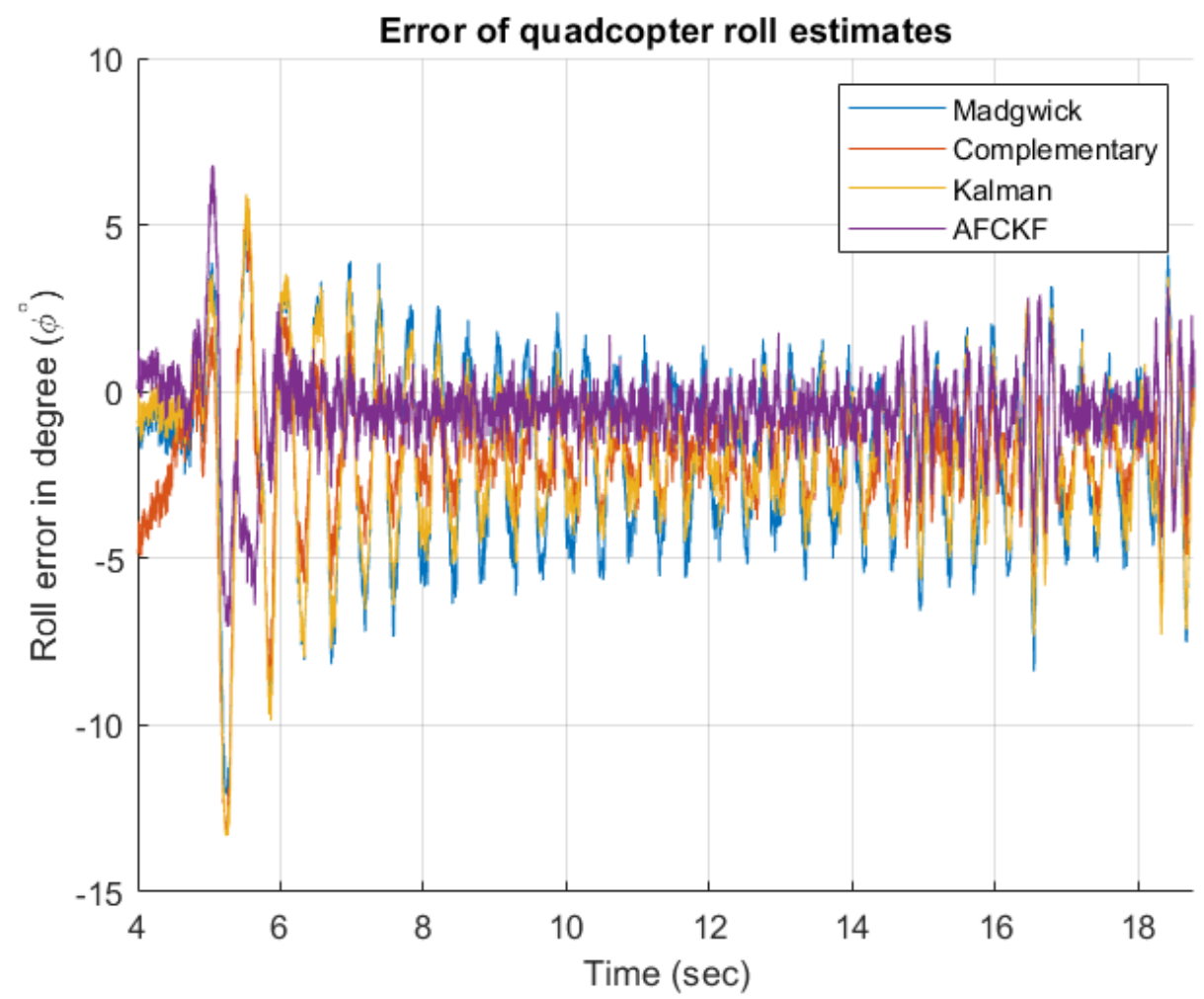

Figure 4.4: Roll estimate error of different filters [Case: Hover with damaged rotor] 
In figures 4.3 and 4.4, it can be observed that although all filters are tracking the roll and pitch estimates, the AFCKF achieves the best tracking result for both. The RMS error, variance and execution time of the different attitude estimators are compared in tables $4.1,4.2$, and 4.3 respectively.

\begin{tabular}{|c|c|c|c|c|}
\hline & Madgwick & Complementary & Kalman & AFCKF \\
\hline Yaw $\left(\psi^{\circ}\right)$ & 143.5094 & 164.8690 & 150.4092 & 38.5072 \\
\hline Pitch $\left(\theta^{\circ}\right)$ & 2.7121 & 2.0417 & 2.4125 & 1.5717 \\
\hline Roll $\left(\phi^{\circ}\right)$ & 3.2761 & 2.7165 & 3.0184 & 1.4599 \\
\hline
\end{tabular}

Table 4.1: RMS error of different attitude estimators [Case: Hover with damaged rotor]

\begin{tabular}{|c|c|c|c|c|}
\hline & Madgwick & Complementary & Kalman & AFCKF \\
\hline Yaw $\left(\psi^{\circ}\right)$ & 20591.4895 & 27182.5512 & 22415.3247 & 1432.8709 \\
\hline Pitch $\left(\theta^{\circ}\right)$ & 6.9058 & 4.0811 & 5.5746 & 1.8775 \\
\hline Roll $\left(\phi^{\circ}\right)$ & 6.9263 & 3.4681 & 5.5789 & 1.7705 \\
\hline
\end{tabular}

Table 4.2: Variance of different attitude estimators [Case: Hover with damaged rotor]

\begin{tabular}{|c|c|c|c|c|}
\hline & Madgwick & Complementary & Kalman & AFCKF \\
\hline Time (sec) & 0.0000708081 & 0.0005973110 & 0.0004862559 & 0.0001110586 \\
\hline
\end{tabular}

Table 4.3: Execution time of different attitude estimators [Case: Hover with damaged rotor]

It can be observed that the attitude estimates obtained using the AFCKF are the best among all the estimators. However, the Madgwick filter is the fastest, with an execution time of about 0.00007 seconds, and thus the least computationally heavy. Compared to the Madgwick filter, the AFCKF is approximately 1.4 times slower, while the Kalman-based filter is approximately five times slower than the AFCKF and the complementary filter is approximately six times slower.

\subsubsection{Hover flight without damaged rotor}

\section{Flight description}

The filter is not only tested for cases with a damaged rotor, but also for the cases in which it works normally. A flight test was conducted with the quadcopter hovering without any linear or angular motion commands. Linear motion and the Euler angles of the quadcopter obtained via the OptiTrack system are presented in figure 4.5. It can be observed that there is no motion along any axis and the Euler angles of the quadcopter also remain constant. 


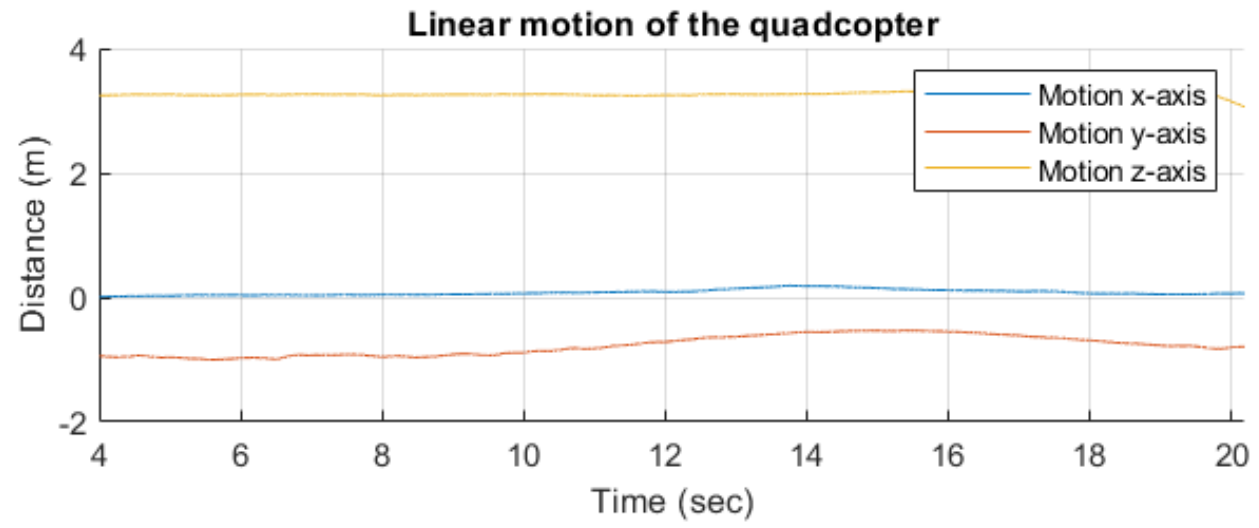

Euler angles of the quadcopter

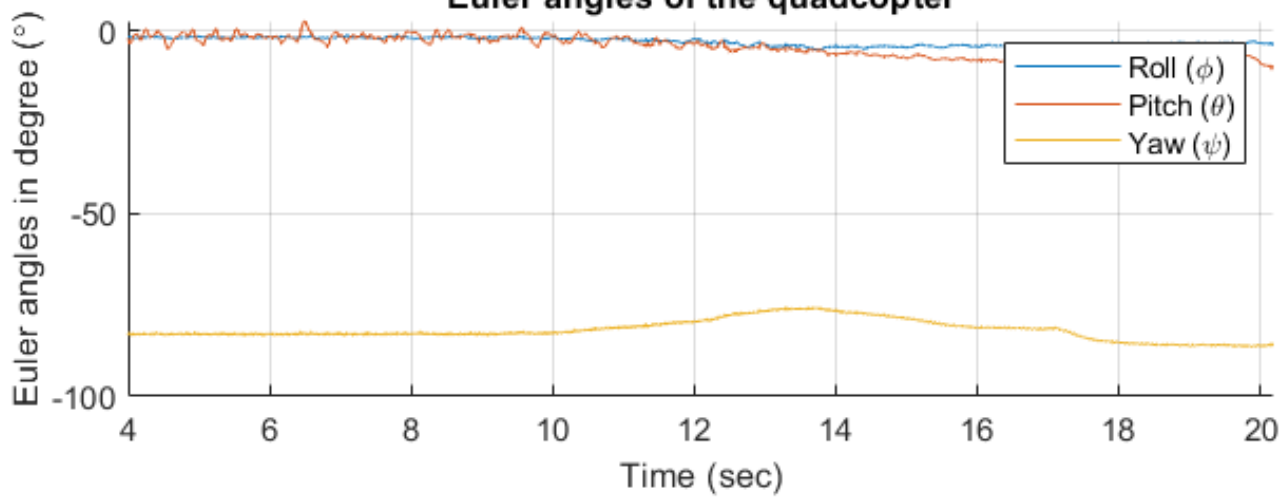

Figure 4.5: Flight path : Hover with all rotor working

\section{Performance of the estimators}

Figures 4.6, 4.7 and 4.8 show the yaw, pitch and roll estimation error of different filters respectively.

The RMS error, variance and execution time of the different attitude estimators are compared in tables $4.4,4.5$, and 4.6 . 


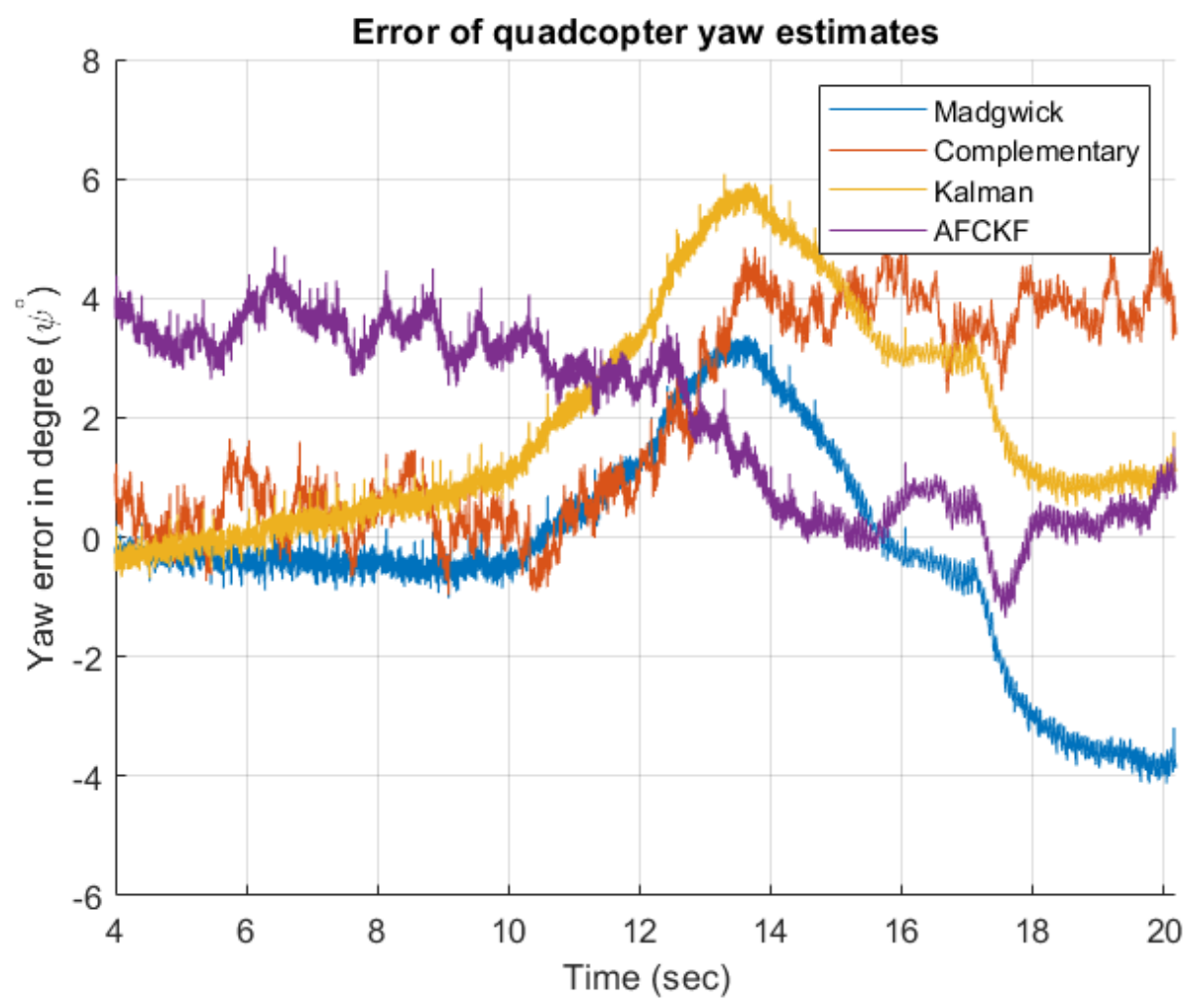

Figure 4.6: Yaw estimate error of different filters [Case: Hover without damaged rotor]

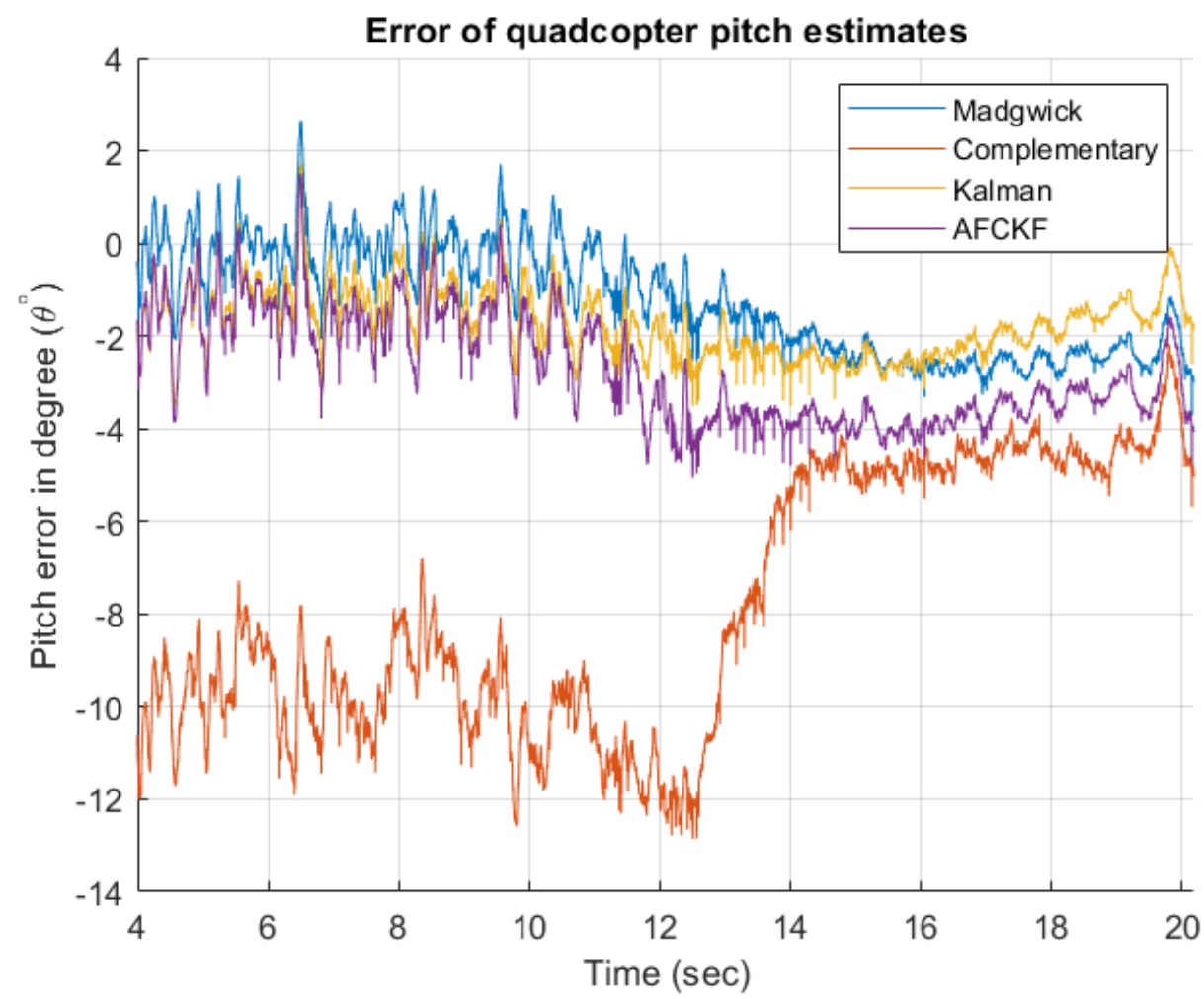

Figure 4.7: Pitch estimate error of different filters [Case: Hover without damaged rotor] 


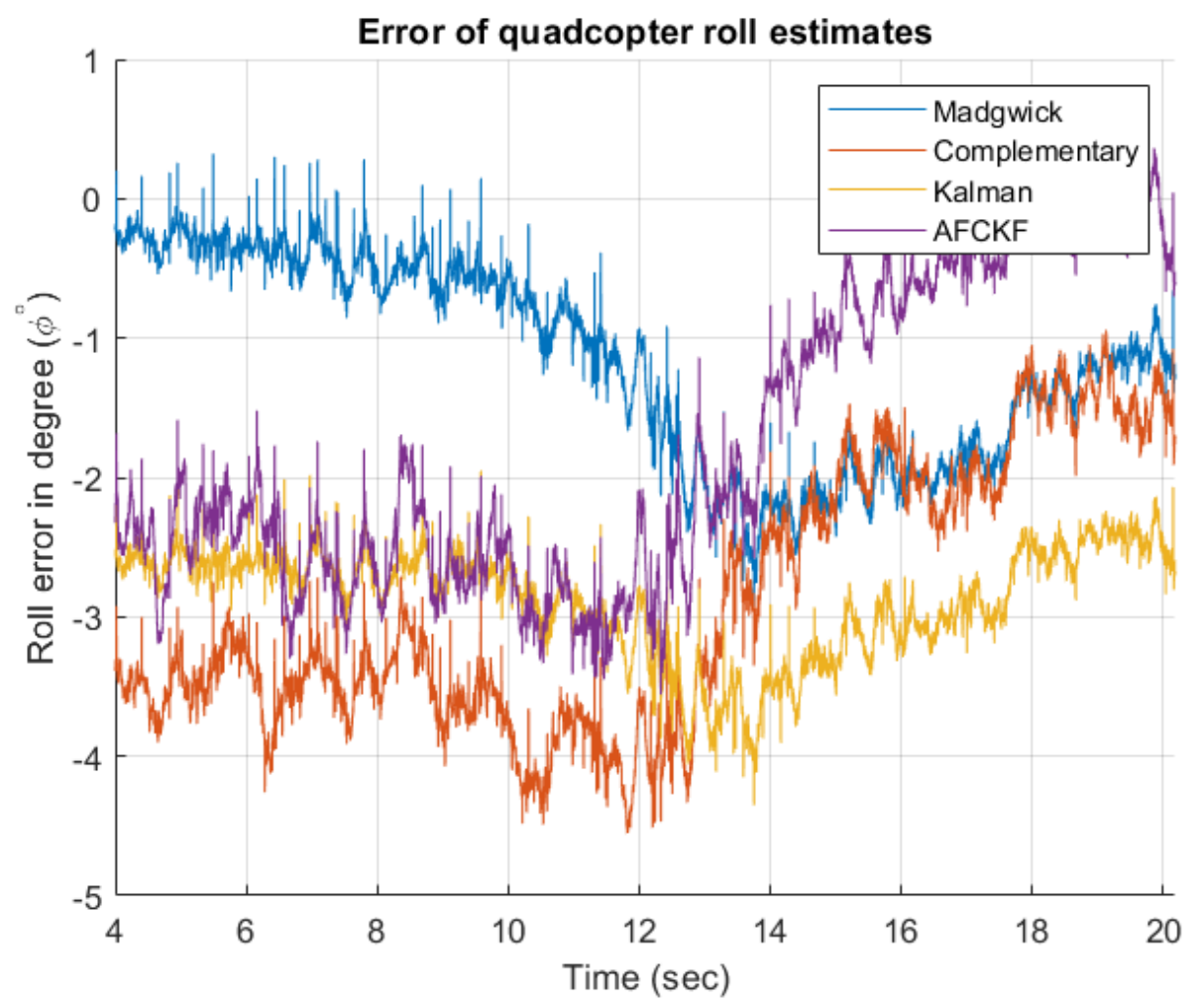

Figure 4.8: Roll estimate error of different filters [Case: Hover without damaged rotor]

\begin{tabular}{|c|c|c|c|c|}
\hline & Madgwick & Complementary & Kalman & AFCKF \\
\hline Yaw $\left(\psi^{\circ}\right)$ & 1.8073 & 2.6526 & 2.6571 & 2.4921 \\
\hline Pitch $\left(\theta^{\circ}\right)$ & 1.6893 & 8.2690 & 1.8590 & 2.0445 \\
\hline Roll $\left(\phi^{\circ}\right)$ & 1.3670 & 3.0333 & 2.9042 & 2.0558 \\
\hline
\end{tabular}

Table 4.4: RMS error of different attitude estimators [Case: Hover without damaged rotor]

\begin{tabular}{|c|c|c|c|c|}
\hline & Madgwick & Complementary & Kalman & AFCKF \\
\hline Yaw $\left(\psi^{\circ}\right)$ & 3.1942 & 3.0433 & 3.3033 & 2.2183 \\
\hline Pitch $\left(\theta^{\circ}\right)$ & 1.4312 & 7.8106 & 0.5657 & 1.3689 \\
\hline Roll $\left(\phi^{\circ}\right)$ & 0.5101 & 0.8680 & 0.1548 & 1.1665 \\
\hline
\end{tabular}

Table 4.5: Variance of different attitude estimators [Case: Hover without damaged rotor]

\begin{tabular}{|c|c|c|c|c|}
\hline & Madgwick & Complementary & Kalman & AFCKF \\
\hline Time (sec) & 0.0000700675 & 0.0005967342 & 0.0004834122 & 0.0001097318 \\
\hline
\end{tabular}

Table 4.6: Average execution time of different attitude estimators [Case: Hover without damaged rotor] 
As can be observed from figures 4.6, 4.7, and 4.8, as well as tables 4.4 and 4.5, all filters are able to track the roll, pitch, and yaw of the quadcopter with high accuracy. There is no significant difference between the performance of different filters. The differences in the execution time are about the same as in the previous case.

\subsubsection{Aggressive motion flight with damaged rotor}

\section{Flight description}

In the third test, the quadcopter was made to move from one point to another with one rotor fully damaged. Linear motion and the Euler angles of the quadcopter obtained via the OptiTrack system are presented in figure 4.9. The quadcopter moved from one point to another while experiencing an extremely high yaw rate of approximately $24 \mathrm{rad} / \mathrm{sec}$ due to the damaged rotor. The flight is highly aggressive in nature, with sudden high values of roll and pitch being given to the quadcopter.
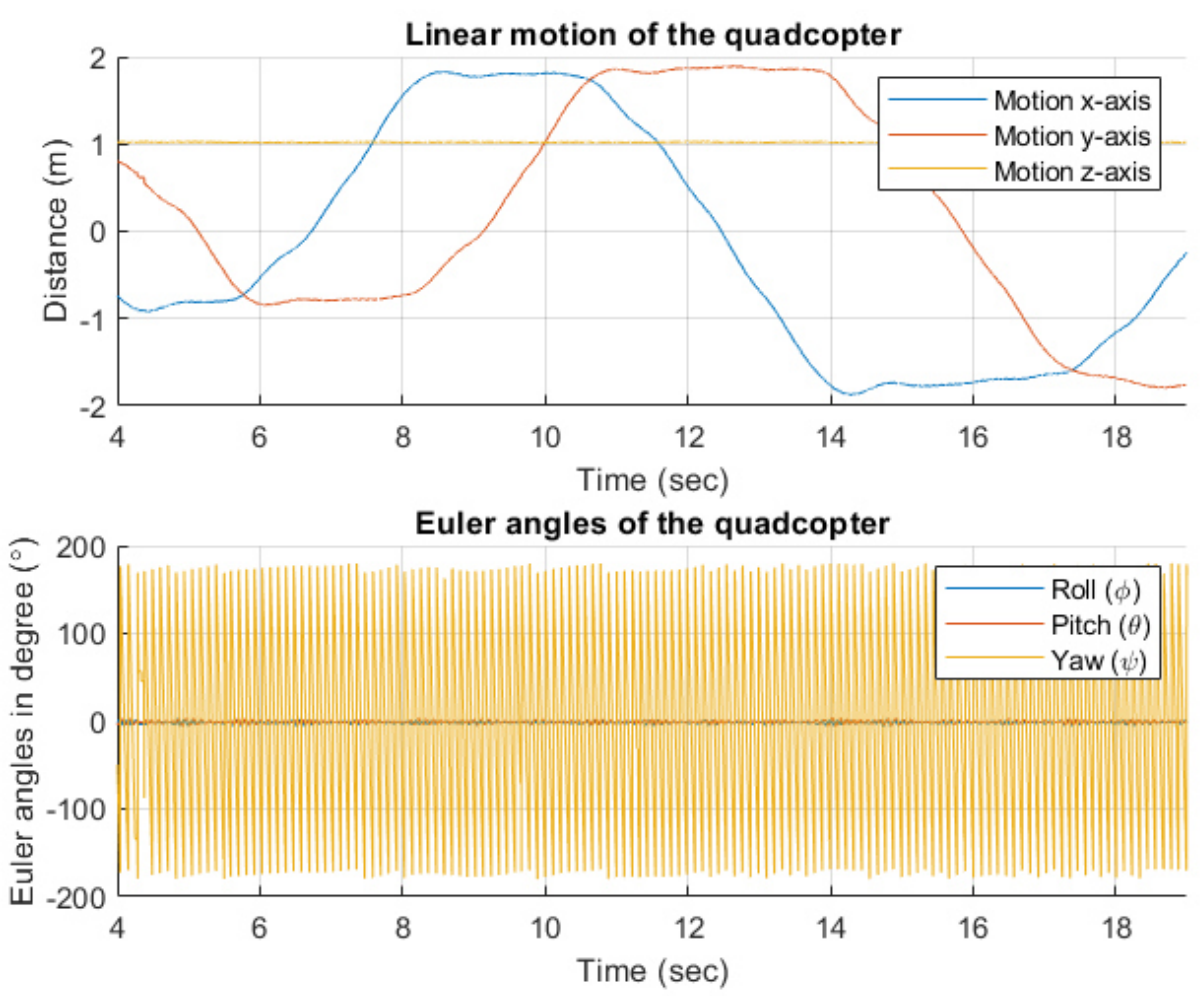

Figure 4.9: Flight path: Motion with damaged rotor

\section{Performance of the estimators}

Figures 4.10, 4.11, and 4.12 show the yaw, pitch and roll estimation error of the different filters respectively, while the RMS error, variance, and execution time of the different attitude estimators are compared in tables $4.7,4.8$, and 4.9 . 


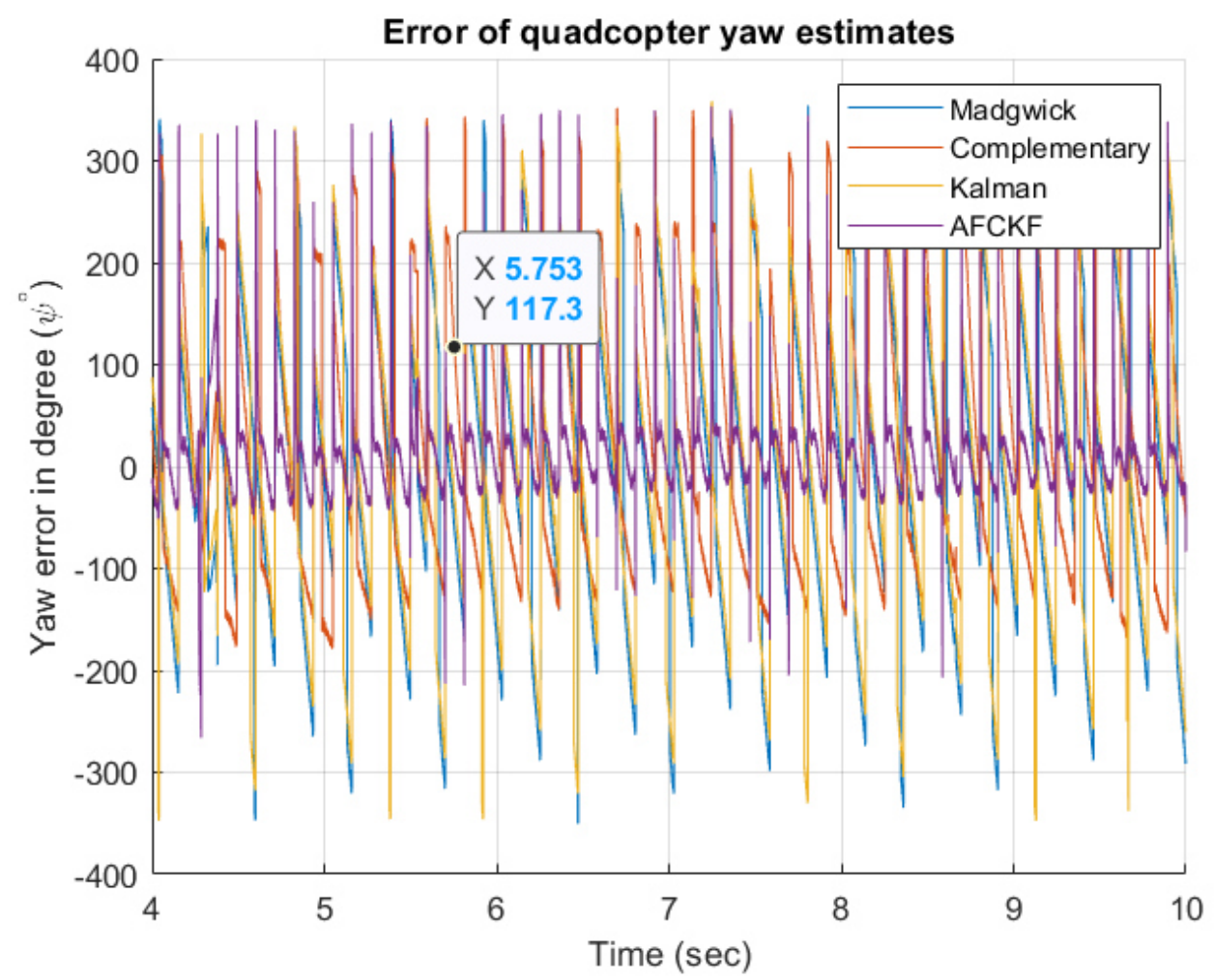

Figure 4.10: Yaw estimate error of different filters [Case: Motion with damaged rotor]

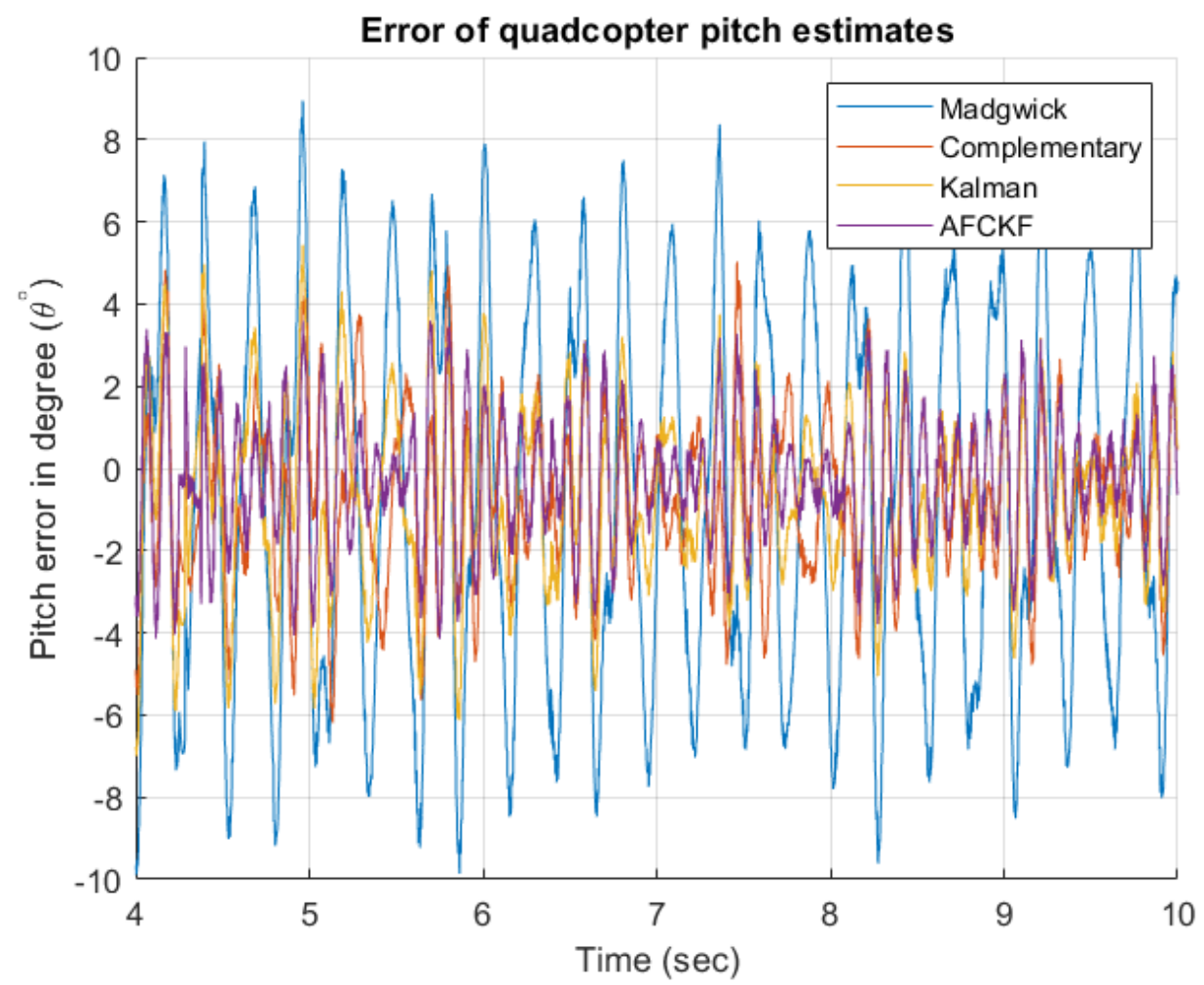

Figure 4.11: Pitch estimate error of different filters [Case: Motion with damaged rotor] 


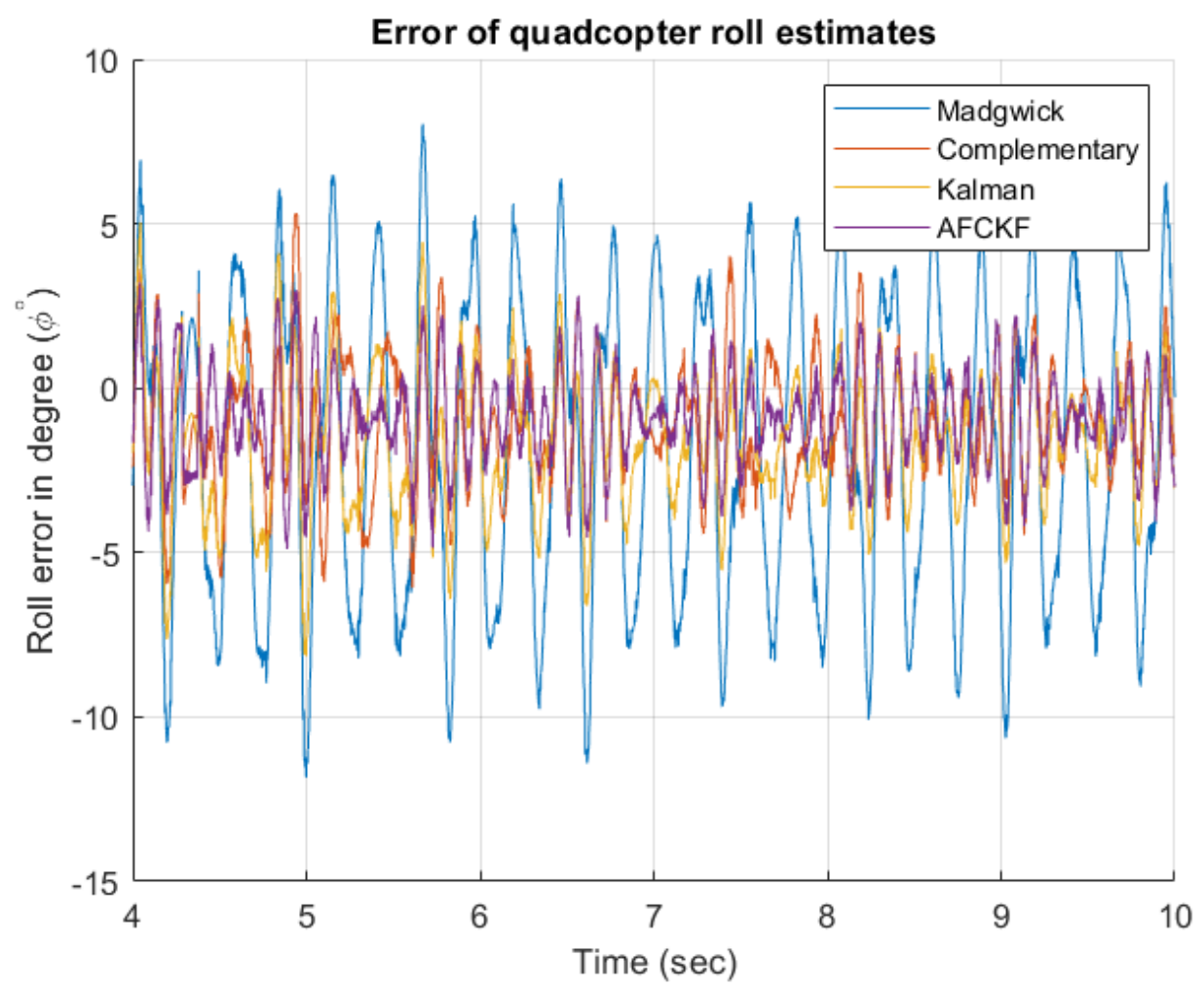

Figure 4.12: Roll estimate error of different filters [Case: Motion with damaged rotor]

\begin{tabular}{|c|c|c|c|c|}
\hline & Madgwick & Complementary & Kalman & AFCKF \\
\hline Yaw $\left(\psi^{\circ}\right)$ & 146.3061 & 141.0257 & 144.3796 & 58.5453 \\
\hline Pitch $\left(\theta^{\circ}\right)$ & 4.7062 & 2.0910 & 2.1813 & 1.5606 \\
\hline Roll $\left(\phi^{\circ}\right)$ & 4.9585 & 2.1326 & 2.5622 & 1.7269 \\
\hline
\end{tabular}

Table 4.7: RMS error of different attitude estimators [Case: Motion with damaged rotor]

\begin{tabular}{|c|c|c|c|c|}
\hline & Madgwick & Complementary & Kalman & AFCKF \\
\hline Yaw $\left(\psi^{\circ}\right)$ & 21409.0225 & 19193.9648 & 20848.3964 & 3196.6480 \\
\hline Pitch $\left(\theta^{\circ}\right)$ & 21.7041 & 3.8913 & 4.3624 & 2.3661 \\
\hline Roll $\left(\phi^{\circ}\right)$ & 21.4318 & 3.5279 & 4.1451 & 2.1017 \\
\hline
\end{tabular}

Table 4.8: Variance of different attitude estimators [Case: Motion with damaged rotor]

\begin{tabular}{|c|c|c|c|c|}
\hline & Madgwick & Complementary & Kalman & AFCKF \\
\hline Time (sec) & 0.0000713500 & 0.0006036904 & 0.0004910872 & 0.0001104184 \\
\hline
\end{tabular}

Table 4.9: Average execution time of different attitude estimators [Case: Motion with damaged rotor] 
Similar to the case of hover flight with a damaged rotor, it can be seen that only the AFCKF is able to track the yaw estimate. As explained before, the peaks seen in the figure stem from the wrapping of the Euler angles. Looking at the roll and pitch estimates, although all estimators are able to track the roll and pitch, the AFCKF outperforms the others. The execution time shows the same results as in the cases above, implying that the computational cost remains the same.

\subsubsection{Aggressive motion flight without damaged rotor}

\section{Flight description}

In the last flight test, the quadcopter was made to move from one point to another with all rotors working perfectly. Linear motion and the Euler angles of the quadcopter obtained via the OptiTrack system are presented in figure 4.13. As before, the flight is highly aggressive in nature, with sudden high values of roll and pitch being given to the quadcopter.
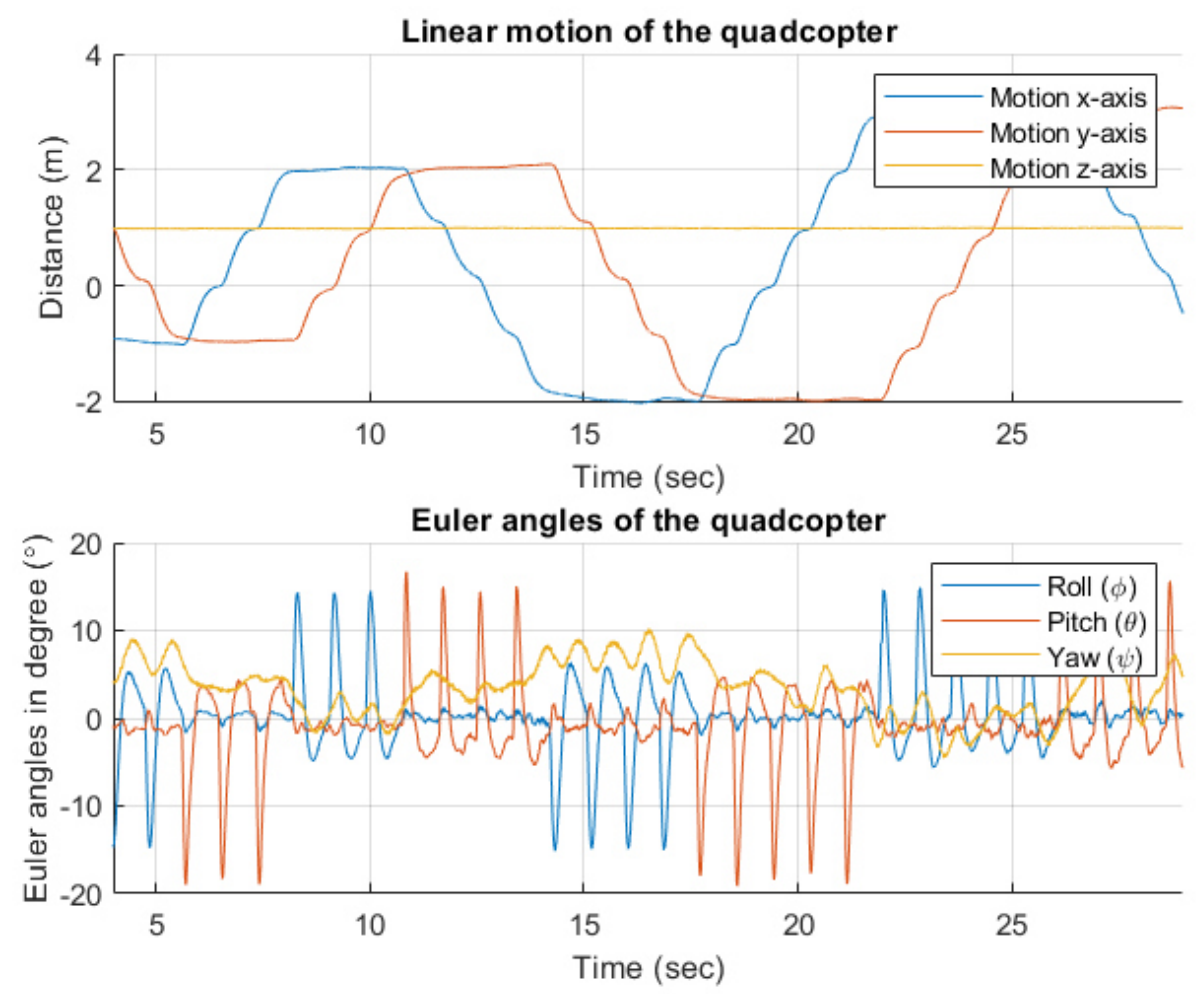

Figure 4.13: Flight path: Motion with working rotor and aggressive maneuver

\section{Estimators performance}

Figures 4.14, 4.15, and 4.16 show the yaw, pitch and roll estimation error of different filters respectively. The RMS error, variance, and execution time of the different attitude estimators are compared in tables 4.10, 4.11, and 4.12. 


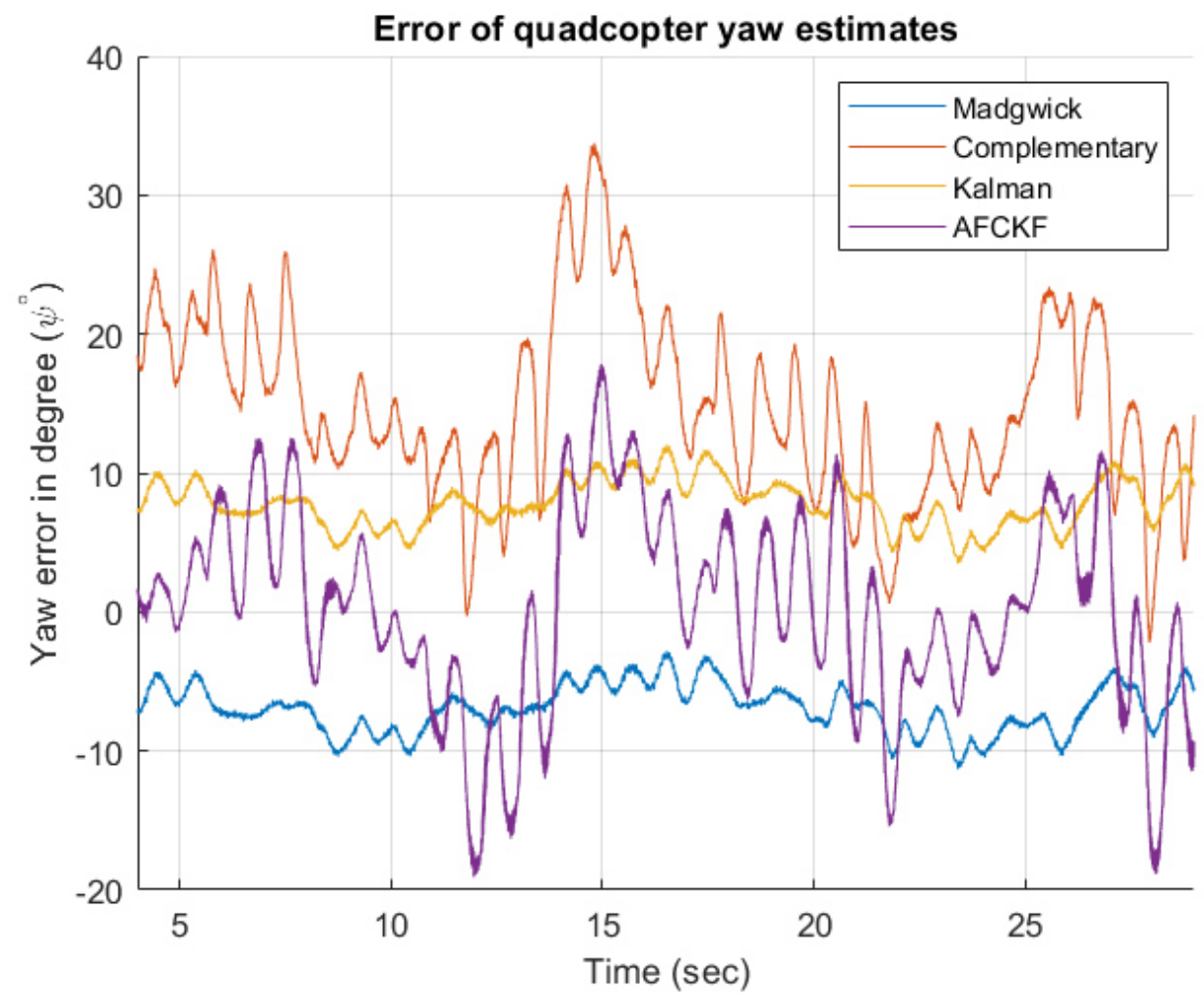

Figure 4.14: Yaw estimate error of different filters [Case: Working rotors and aggressive maneuver]

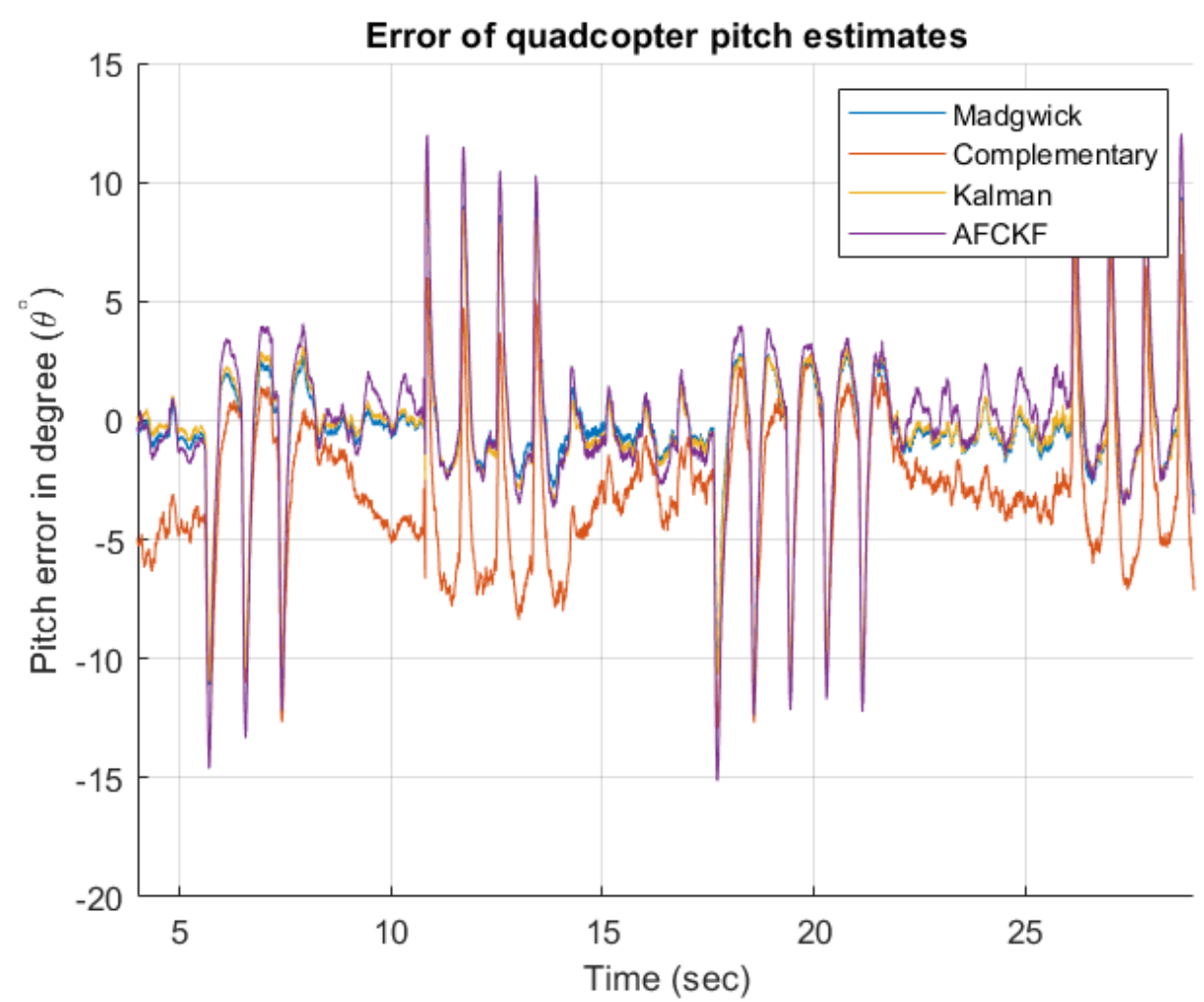

Figure 4.15: Pitch estimate error of different filters [Case: Working rotors and aggressive maneuver] 


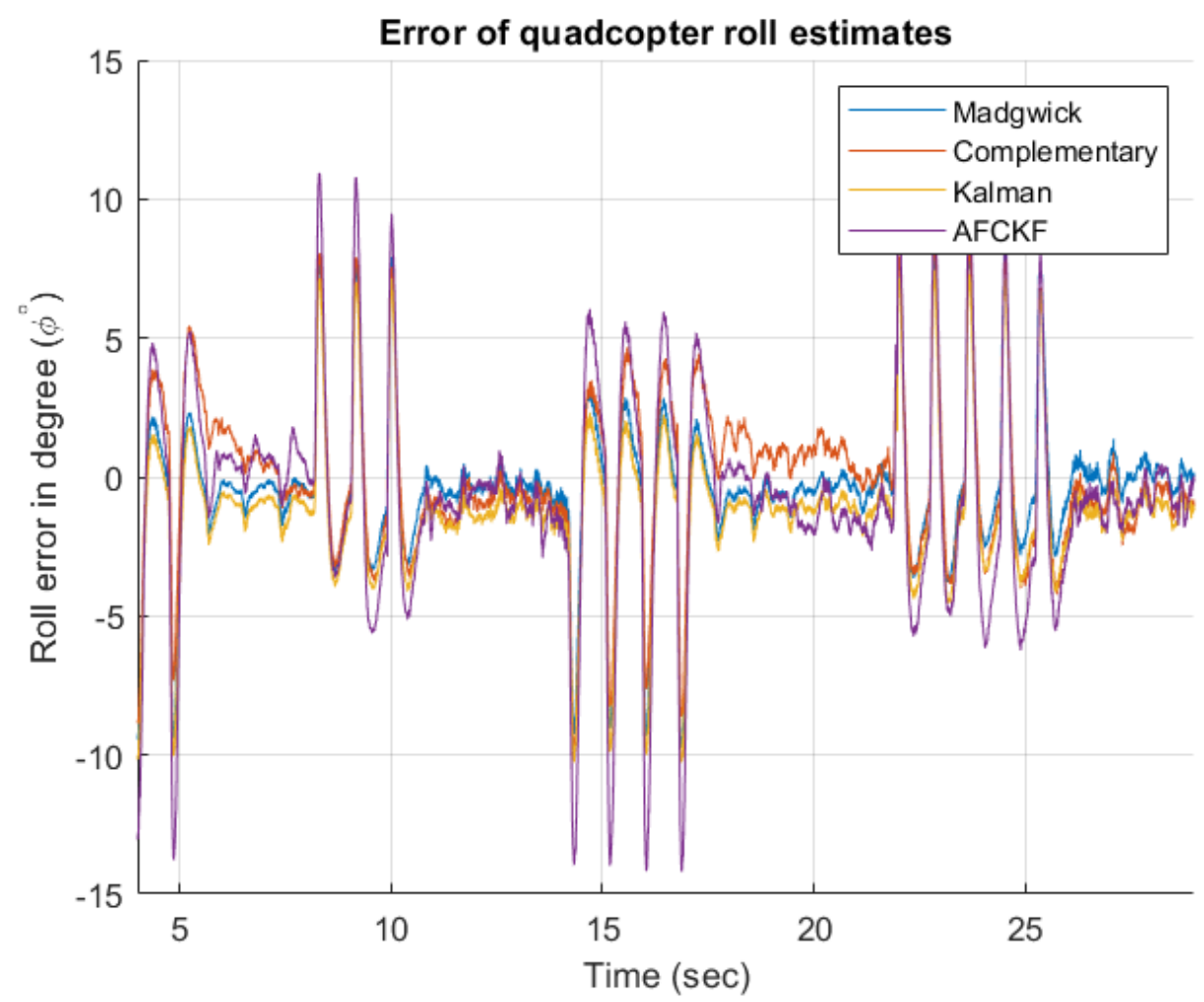

Figure 4.16: Roll estimate error of different filters [Case: Working rotors and aggressive maneuver]

\begin{tabular}{|c|c|c|c|c|}
\hline & Madgwick & Complementary & Kalman & AFCKF \\
\hline Yaw $\left(\psi^{\circ}\right)$ & 7.1113 & 16.2907 & 8.0117 & 7.0631 \\
\hline Pitch $\left(\theta^{\circ}\right)$ & 2.7577 & 4.3293 & 2.7746 & 2.8609 \\
\hline Roll $\left(\phi^{\circ}\right)$ & 2.5452 & 2.7714 & 2.7852 & 2.9435 \\
\hline
\end{tabular}

Table 4.10: RMS error of different attitude estimators [Case: Working rotors and aggressive maneuver]

\begin{tabular}{|c|c|c|c|c|}
\hline & Madgwick & Complementary & Kalman & AFCKF \\
\hline Yaw $\left(\psi^{\circ}\right)$ & 2.8549 & 42.7581 & 2.8042 & 49.7615 \\
\hline Pitch $\left(\theta^{\circ}\right)$ & 9.4771 & 10.5227 & 9.6016 & 11.9753 \\
\hline Roll $\left(\phi^{\circ}\right)$ & 10.3513 & 11.6798 & 10.3800 & 14.3129 \\
\hline
\end{tabular}

Table 4.11: Variance of different attitude estimators [Case: Working rotors and aggressive maneuver]

\begin{tabular}{|c|c|c|c|c|}
\hline & Madgwick & Complementary & Kalman & AFCKF \\
\hline Time (sec) & 0.0000707470 & 0.0006009932 & 0.0004862533 & 0.0001096870 \\
\hline
\end{tabular}

Table 4.12: Average execution time of different attitude estimators [Case: Working rotors and aggressive maneuver] 
As can be observed from figures 4.14. 4.15, and 4.16, as well as tables 4.10 and 4.11, all filters are able to track the roll, pitch, and yaw of the quadcopter with a high accuracy. There is no significant difference between the filters' performances. Furthermore it can be observed from table 4.12 that the execution time is about the same as in the previous cases.

To sum up, it can be seen from the results that in the cases in which one rotor is fully damaged and the quadcopter is spinning rigorously about the yaw axis, the AFCKF estimates the roll and pitch of the quadcopter much more accurate than the other estimators, with an RMS error of less than 1.7 degrees and a variance of less than 2 degrees. For the yaw estimate, although it can be seen that as the yaw rate increases, the estimation error increases as well, it is the only filter that is able to track the quadcopter's yaw angle. In the cases in which the rotor is not damaged, all filters show roughly similar performances with small differences that do not point to the dominance of any individual filter. Looking at the execution time, it can be observed that the Madgwick Filter is the fastest in all cases, while the AFCKF is on average 1.4 times slower than the Madgwick filter. However, it is still on average five times faster than the EKF-based filter and about six times faster than the complex complementary filter.

One drawback of the AFCKF is that if there are long-term magnetic distortions, the filter's yaw estimates will diverge from the real yaw estimates. The fuzzy gain can only compensate short-term magnetic field distortions. However, in outdoor environments (which this filter is designed for) it is highly unlikely that there is a long-term magnetic distortion. All in all, it can be observed that the AFCKF performs well and, if a rotor failure occurs during an outdoor flight, it will be able to provide accurate estimates so that the controller should be able to stabilize the quadcopter's flight.

\subsubsection{Comparison of the additive and multiplicative versions of the AFCKF}

As explained earlier, both the additive and multiplicative versions of the AFCKF are providing similar results with only negligible differences. This is evidenced by figures 4.17, 4.18, and 4.19, which show the comparison for the case of hover flight with a damaged rotor. The comparisons for the other cases have similar results and can be found in appendix C. It can also be observed that the yaw estimates are exactly the same in both the additive and the multiplicative version, which is because they are obtained via a different channel, as explained above. 


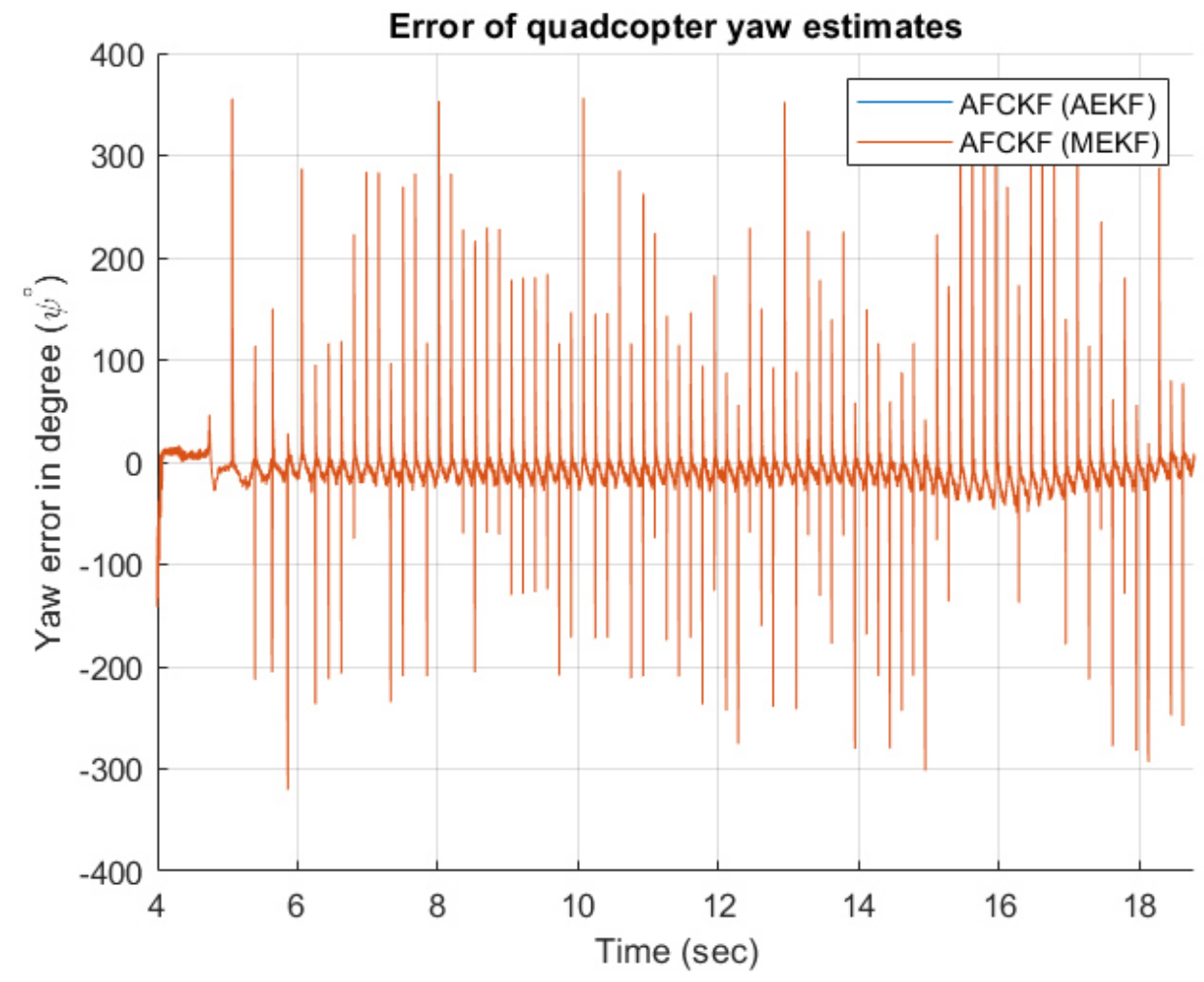

Figure 4.17: Yaw estimate error of additive and multiplicative versions of the AFCKF

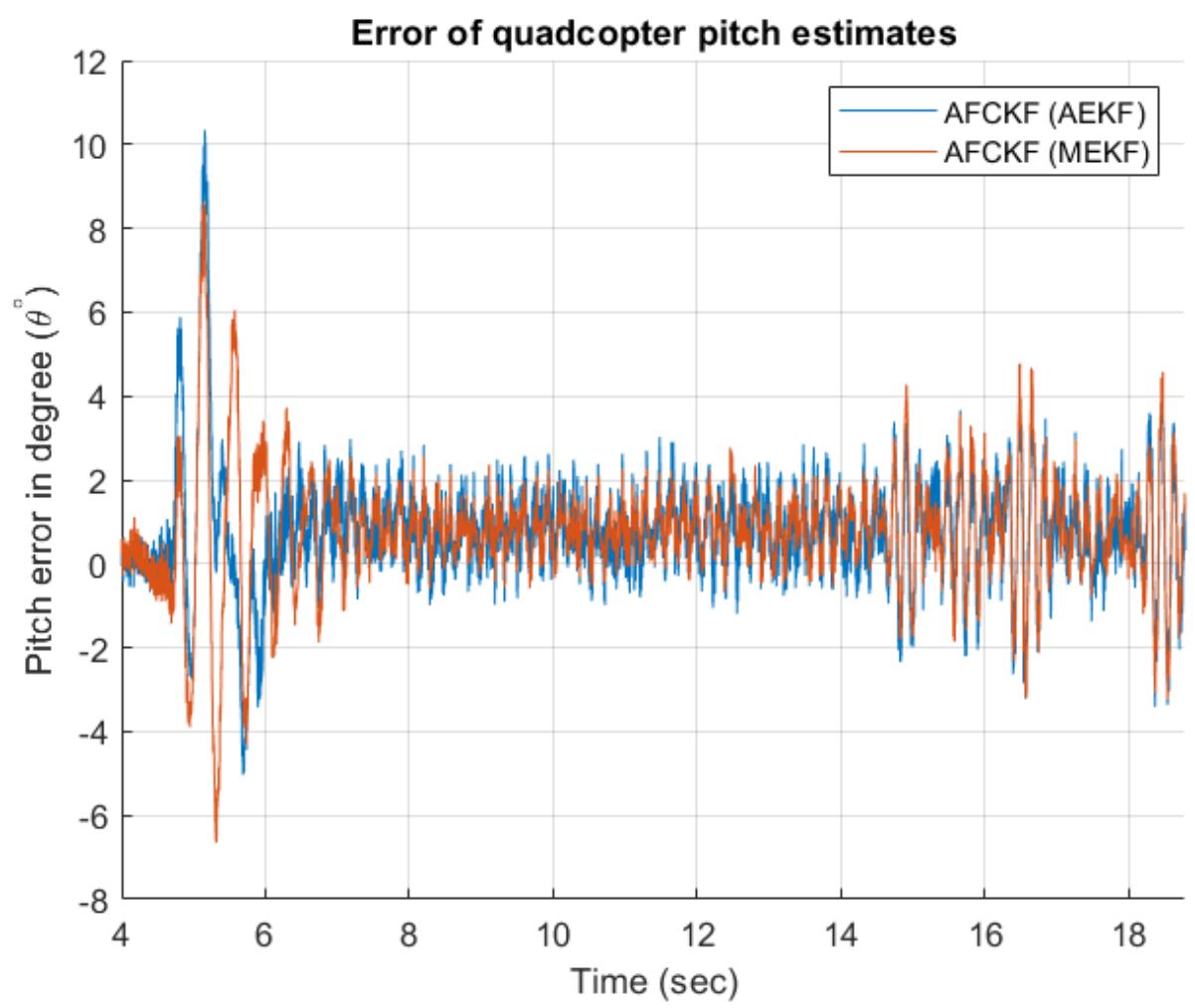

Figure 4.18: Pitch estimate error of additive and multiplicative versions of the AFCKF 


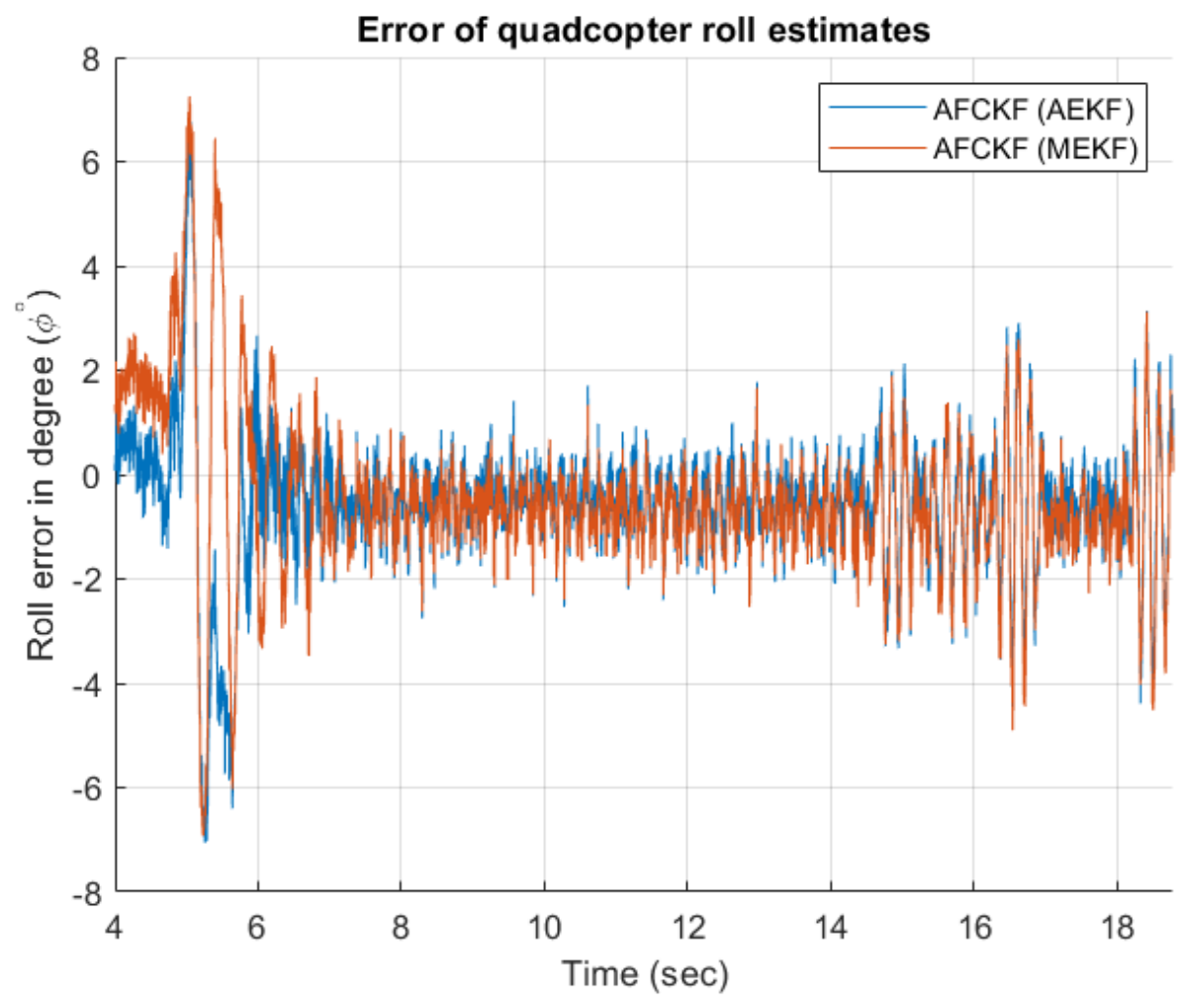

Figure 4.19: Roll estimate error of additive and multiplicative versions of the AFCKF

\section{Conclusion}

In this paper, an attitude estimator that is able to estimate the attitude of a quadcopter with one fully damaged rotor has been developed, validated, and compared to a set of mainstream estimators to evaluate its performance. Quadcopters are valued for their versatility and are getting increasingly common across various fields such as surveillance, delivery, photography and others. Rotor damages occur frequently, which is why research that improves the attitude estimation and thus ultimately the ability to control a damaged quadcopter is of high importance in the field. Once a quadcopter's rotor is damaged, it goes through a highly dynamic motion - especially along the yaw axis - to maintain stability about the roll and pitch axes. This motion contributes to the external acceleration noise of the accelerometer. Because of that, the on-board MARG sensor used in low-cost devices is plagued with high noise and bias. Apart from that, the rate gyroscope bias tends to drift with time due to a change in the temperature of the sensor and the motion of the quadcopter. The magnetometer readings are also affected by the external magnetic field.

To cater for these problems and fill a gap in the existing research, the AFCKF is designed in such a way that the yaw estimates are obtained via a separate channel than the roll and pitch estimates. This prevents the yaw estimation errors from affecting the roll and pitch estimates, which is important because these estimates are essential for the quadcopter's stability. The AFCKF 
caters for the problem of the centrifugal acceleration by using a predictive model. The centrifugal acceleration is predicted using the model and is then subtracted from the accelerometer readings. Furthermore, the accelerometer noise characteristics are adapted to cater for any remaining external accelerations (linear accelerations of the quadcopter). A model is not required to accommodate these linear accelerations because they are short-lived and can be removed by adapting the accelerometer noise characteristics.

The roll and pitch estimates are obtained from the corrected accelerometer readings by using a minimisation scheme. For that, an adaptive gradient descent algorithm is utilised. The algorithm minimises the error between the accelerometer readings and the projection of the gravity vector in the sensor frame. Minimising this function provides the attitude estimates. These estimates are further fused with the roll and pitch estimates obtained from integrating the rate gyro readings. A Kalman filter - additive or multiplicative - is used for fusing the two estimates and consequently providing the optimum roll and pitch estimates. The yaw estimates are obtained through a fuzzy complementary filter, which functions by summing the yaw estimates obtained through integrating the rate gyroscope's yaw estimates and the yaw estimates obtained from the magnetometer readings through a fuzzy gain. The filter gain is obtained using fuzzy logic in order to incorporate external magnetic noise and rate gyro saturation.

The AFCKF was validated using real-time flight data of the quadcopter. The attitude estimates obtained via the AFCKF were validated against the real-time attitude estimates obtained from the OptiTrack motion capture system. Additionally, the AFCKF was also compared against three widely used attitude estimation algorithms in order to examine its relative performance. Four different types of flight tests were conducted: hover flight, hover with damaged rotor, aggressive motion flight, and aggressive motion flight with damaged rotor. As the results show, the AFCKF is able to estimate the quadcopter's attitude as accurate as the other filters for the cases of nondamaged rotor flights, and has a significant better performance than the other three filters for the cases in which the rotor is damaged. For the latter case, the AFCKF estimates the roll and pitch of the quadcopter with an RMS error of less than 1.7 degrees and a variance of less than 2 degrees. For the yaw estimate, although it can be seen that as the yaw rate increases, the estimation error increases as well, it is the only filter that is able to track the quadcopter's yaw angle. For the case without damaged rotor, the AFCKF has an RMS yaw error of less than 2.5 degrees and roll and pitch RMS errors of about 2 degrees. It has a variance of less than 2.3 degrees (yaw) and less than 1.4 (roll and pitch) respectively.

There are a number of future research objectives that can be inspired by the present research. Firstly, in case of a quadcopter damage, it might happen that the inertia matrix of the quadcopter changes, for example if the rotor falls off. Thus, an online parameter estimation scheme might be needed to update the controller to be able to control the damaged quadcopter. Secondly, the attitude estimates can further be improved by using a quadcopter model to predict the linear 
accelerations and subtracting them from the accelerometer readings. Lastly, an online identification scheme can be added, so that the centrifugal acceleration model is improved by the quadcopter during the flight. 


\section{References}

A. J. Baerveldt and R. Klang. Low-cost and low-weight attitude estimation system for an autonomous helicopter. IEEE International Conference on Intelligent Engineering Systems, Proceedings, INES, pages 391-395, 1997. 10.1109/ines.1997.632450.

A. Battiston, I. Sharf, and M. Nahon. Attitude estimation for normal flight and collision recovery of a quadrotor uav. pages 840-849, 06 2017. 10.1109/ICUAS.2017.7991468.

I. Beg and S. Ashraf. Similarity measures for fuzzy sets. Applied and computational mathematics, 8(2):192-202, 2009.

J. Bortz. A new mathematical formulation for strapdown inertial navigation. IEEE Transactions on Aerospace and Electronic Systems, AES-7(1):61-66, 1971. 10.1109/taes.1971.310252.

R. Burton, S. Rock, J. Springmann, and J. Cutler. Online attitude determination of a passively magnetically stabilized spacecraft. Advances in the Astronautical Sciences, 148:2497-2514, 01 2013. 10.1016/j.actaastro.2017.01.024.

J. Calusdian, X. Yun, and E. Bachmann. Adaptive-gain complementary filter of inertial and magnetic data for orientation estimation. Proceedings - IEEE International Conference on Robotics and Automation, pages 1916-1922, 2011. ISSN 10504729. 10.1109/ICRA.2011.5979957.

J. A. Christian and E. G. Lightsey. Sequential optimal attitude recursion filter. Journal of Guidance, Control, and Dynamics, 33(6):1787-1800, 2010. 10.2514/1.49561.

J. M. Cooke, M. J. Zyda, D. R. Pratt, and R. B. McGhee. Npsnet: Flight simulation dynamic modeling using quaternions. Presence: Teleoperators and Virtual Environments, 1(4):404-420, 1992. 10.1162/pres.1992.1.4.404.

E. Cox. Fuzzy fundamentals. IEEE Spectrum, 29(10):58-61, 1992. 10.1109/6.158640.

J. L. Crassidis, F. L. Markley, and Y. Cheng. Survey of nonlinear attitude estimation methods. Journal of Guidance, Control, and Dynamics, 30(1):12-28, 2007. 10.2514/1.22452.

M. B. Del Rosario, N. H. Lovell, and S. J. Redmond. Quaternion-Based Complementary Filter for Attitude Determination of a Smartphone. IEEE Sensors Journal, 16(15):6008-6017, 2016. ISSN 1530437X. 10.1109/JSEN.2016.2574124.

M. Eisele, K. Hentschel, and T. Kunemund. Hardware realization of fast defuzzification by adaptive integration, page 318-323. Proceedings of the Fourth International Conference on Microelectronics for Neural Networks and Fuzzy Systems, 1994.

K. Feng, J. Li, X. Zhang, C. Shen, Y. Bi, T. Zheng, and J. Liu. A new quaternion-based kalman filter for real-time attitude estimation using the two-step geometrically-intuitive correction algorithm. Sensors (Switzerland), 17(9), 2017. ISSN 14248220. 10.3390/s17092146. 
E. Foxlin. Inertial head-tracker sensor fusion by a complementary separate-bias Kalman filter. Proceedings - Virtual Reality Annual International Symposium, pages 185-194, 1996. 10.1109/ vrais.1996.490527.

D. Gebre-Egziabher, R. Hayward, and J. Powell. Design of multi-sensor attitude determination systems. IEEE Transactions on Aerospace and Electronic Systems, 40(2):627-649, 2004. 10.1109/ taes.2004.1310010.

P. C. Hughes. Spacecraft Attitude Dynamics. Dover Publications, 2012.

M. B. Ignagni. Errata: Optimal strapdown attitude integration algorithms. Journal of Guidance, Control, and Dynamics, 13(3):0576b-0576b, 1990. 10.2514/3.56519.

D. Jiang, X. Deng, J. Wu, S. Mo, and Y. Yang. The application of improved extended kalman filter algorithm in satellite attitude determination. 01 2016. 10.2991/wartia-16.2016.277.

D. Jurman, M. Jankovec, R. Kamnik, and M. Topič. Calibration and data fusion solution for the miniature attitude and heading reference system. Sensors and Actuators A: Physical, 138(2): 411-420, 2007. 10.1016/j.sna.2007.05.008.

J. B. Kuipers. Quaternions and rotation sequences: A Primer with Applications to Orbits, Aerospace and Virtual Reality. Princeton University Press, 1999.

W. V. Leekwijck and E. E. Kerre. Defuzzification: criteria and classification. Fuzzy Sets and Systems, 108(2):159-178, 1999. 10.1016/s0165-0114(97)00337-0.

E. Lefferts, L. Markley, and M. Shuster. Kalman filtering for spacecraft attitude estimation. Journal of Guidance, Control, and Dynamics, 5, 02 1982. 10.2514/3.56190.

B. Liu, Z. Chen, L. Xiangdong, and F. Yang. An efficient nonlinear filter for spacecraft attitude estimation. International Journal of Aerospace Engineering, 2014:1-11, 03 2014. 10.1155/2014/ 540235 .

D. P. Madau and L. Feldkamp. Influence value defuzzification method. Fuzzy Systems, 3:1819-1824, 1996.

S. O. Madgwick, A. J. Harrison, and R. Vaidyanathan. Estimation of IMU and MARG orientation using a gradient descent algorithm. IEEE International Conference on Rehabilitation Robotics, pages 1-7, 2011. ISSN 19457898. 10.1109/ICORR.2011.5975346.

R. Mahony, T. Hamel, and J. M. Pflimlin. Nonlinear complementary filters on the special orthogonal group. IEEE Transactions on Automatic Control, 53(5):1203-1218, 2008. ISSN 00189286. 10.1109/TAC.2008.923738. 
J. L. Marins, X. Yun, E. R. Bachmann, R. B. McGhee, and M. J. Zyda. An extended Kalman filter for quaternion-based orientation estimation using MARG sensors. IEEE International Conference on Intelligent Robots and Systems, 4:2003-2011, 2001. 10.1109/iros.2001.976367.

F. Markley. Attitude determination and parameter estimation using vector observations. Journal of the Astronautical Sciences, 36(6):245-258, 1988. 10.2514/6.1988-4225.

L. Markley. Multiplicative vs. additive filtering for spacecraft attitude determination. 072004.

D. Mortari. Esoq: A closed-form solution to the wahba problem. Journal of the Astronautical Sciences, 45(2):195-204, 1997.

M. W. Mueller and R. D'Andrea. Stability and control of a quadrocopter despite the complete loss of one, two, or three propellers. Proceedings - IEEE International Conference on Robotics and Automation, pages 45-52, 2014. ISSN 10504729. 10.1109/ICRA.2014.6906588.

M. W. Mueller and R. D'Andrea. Relaxed hover solutions for multicopters: Application to algorithmic redundancy and novel vehicles. International Journal of Robotics Research, 35(8): 873-889, 2016. ISSN 17413176. 10.1177/0278364915596233.

F. Qin, L. Chang, S. Jiang, and F. Zha. A sequential multiplicative extended kalman filter for attitude estimation using vector observations. Sensors, 18:1414, 05 2018. 10.3390/s18051414.

D. Roetenberg, H. Luinge, C. Baten, and P. Veltink. Compensation of magnetic disturbances improves inertial and magnetic sensing of human body segment orientation. IEEE Transactions on Neural Systems and Rehabilitation Engineering, 13(3):395-405, 2005. 10.1109/tnsre.2005 .847353 .

A. M. Sabatini. Quaternion-based extended Kalman filter for determining orientation by inertial and magnetic sensing. IEEE Transactions on Biomedical Engineering, 53(7):1346-1356, 2006. ISSN 00189294. 10.1109/TBME.2006.875664.

S. Shan, Z. Hou, and J. Wu. Linear Kalman Filter for Attitude Estimation from Angular Rate and a Single Vector Measurement. Journal of Sensors, 2017, 2017. ISSN 16877268. 10.1155/ $2017 / 9560108$.

M. Shuster. Constraint in attitude estimation part i: Constrained estimation. Journal of the Optical Society of America, 51, 03 2003a. 10.1007/BF03546315.

M. Shuster. Constraint in attitude estimation part ii: Unconstrained estimation. Journal of the Optical Society of America, 51, 03 2003b. 10.1007/BF03546316.

M. D. Shuster. A survey of attitude representation. Journal of the Astronautical Sciences, 41(4): 439-517, 1993. 
M. D. Shuster and S. D. Oh. Three-axis attitude determination from vector observation. Journal of Guidance, Control, and Dynamics, 4(1):70-77, 1981.

J. Stuelpnagel. On the parametrization of the three-dimensional rotation group. SIAM Review, 6 (4):422-430, 1964. 10.1137/1006093.

S. Sun, R. Schilder, and C. De Visser. Identification of Quadrotor Aerodynamic Model from High Speed Flight Data. AIAA Atmospheric Flight Mechanics Conference, 2018a. 10.2514/6.2018 -0523 .

S. Sun, L. Sijbers, X. Wang, and C. De Visser. High-Speed Flight of Quadrotor Despite Loss of Single Rotor. IEEE Robotics and Automation Letters, 3(4):3201-3207, 2018b. ISSN 23773766. 10.1109/LRA.2018.2851028.

Q. Tao et al. Error compensation method for electronic compass based on best ellipse-matching error compensation algorithm. Chinese Journal of Sensors and Actuators, 11:1499-1503, 2013.

Y. Tian, H. Wei, and J. Tan. An adaptive-gain complementary filter for real-time human motion tracking with MARG sensors in free-living environments. IEEE Transactions on Neural Systems and Rehabilitation Engineering, 21(2):254-264, 2013. ISSN 15344320. 10.1109/TNSRE.2012 .2205706 .

D. H. Titterton and J. L. Weston. Strapdown inertial navigation technology. Institution of Electrical Engineers, 2004.

R. Valenti, I. Dryanovski, and J. Xiao. Keeping a good attitude: A quaternion-based orientation filter for imus and margs. Sensors, 15(8):19302-19330, 2015. 10.3390/s150819302.

G. Wahba. Problem 65-1: A least squares estimate of satellite attitude. SIAM Review, 7(3):409, 1966.

L. Wang, Z. Zhang, and P. Sun. Quaternion-Based Kalman Filter for AHRS Using an AdaptiveStep Gradient Descent Algorithm. International Journal of Advanced Robotic Systems, 12(9): 1-12, 2015. ISSN 17298814. 10.5772/61313.

J. Wu, Z. Zhou, J. Chen, H. Fourati, and R. Li. Fast Complementary Filter for Attitude Estimation Using Low-Cost MARG Sensors. IEEE Sensors Journal, 16(18):6997-7007, 2016. ISSN 1530437X. 10.1109/JSEN.2016.2589660.

Y. Xiaoping, C. Aparicio, E. R. Bachmann, and R. B. McGhee. Implementation and experimental results of a quaternion-based Kalman filter for human body motion tracking. Proceedings - IEEE International Conference on Robotics and Automation, 2005(6):317-322, 2005. ISSN 10504729. 10.1109/ROBOT.2005.1570138. 
L. Xing, Y. Hang, Z. Xiong, J. Liu, and Z. Wan. Accurate attitude estimation using ARS under conditions of vehicle movement based on disturbance acceleration adaptive estimation and correction. Sensors (Switzerland), 16(10), 2016. ISSN 14248220. 10.3390/s16101716.

N. Yazdi, F. Ayazi, and K. Najafi. Micromachined inertial sensors. Proceedings of the IEEE, 86 (8):1640-1659, 1998. 10.1109/5.704269.

X. Yuan, S. Yu, S. Zhang, G. Wang, and S. Liu. Quaternion-based unscented kalman filter for accurate indoor heading estimation using wearable multi-sensor system. Sensors (Switzerland), 15(5):10872-10890, 2015. ISSN 14248220. 10.3390/s150510872. 


\section{APPENDIX}

\section{A All results}

A.1 All results for the case of hover flight without damaged rotors

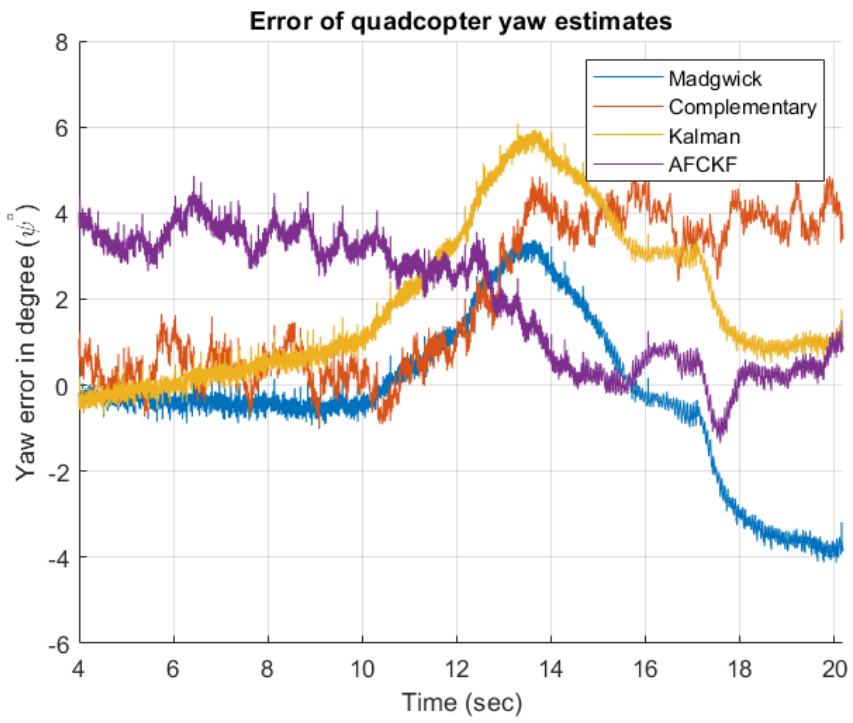

Figure A.1: Yaw estimate error of different filters [Case: Hover without damaged rotor]

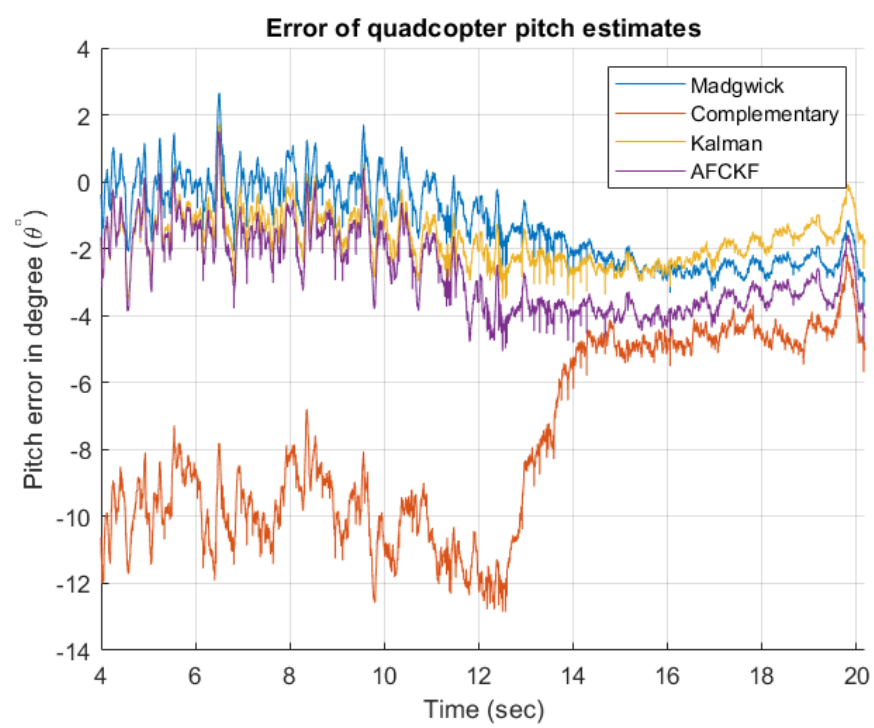

Figure A.2: Pitch estimate error of different filters [Case: Hover without damaged rotor] 


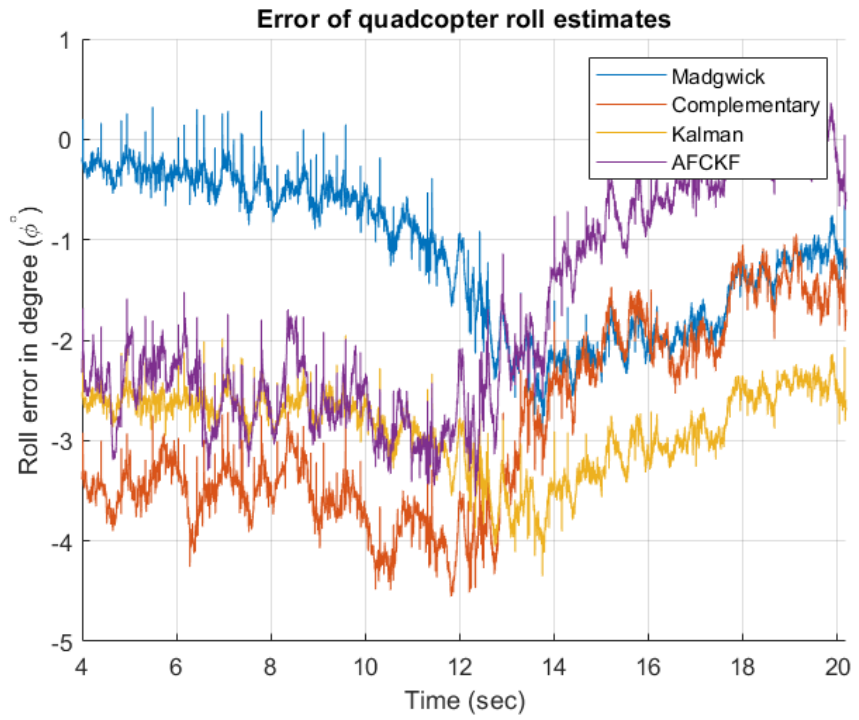

Figure A.3: Roll estimate error of different filters [Case: Hover without damaged rotor]

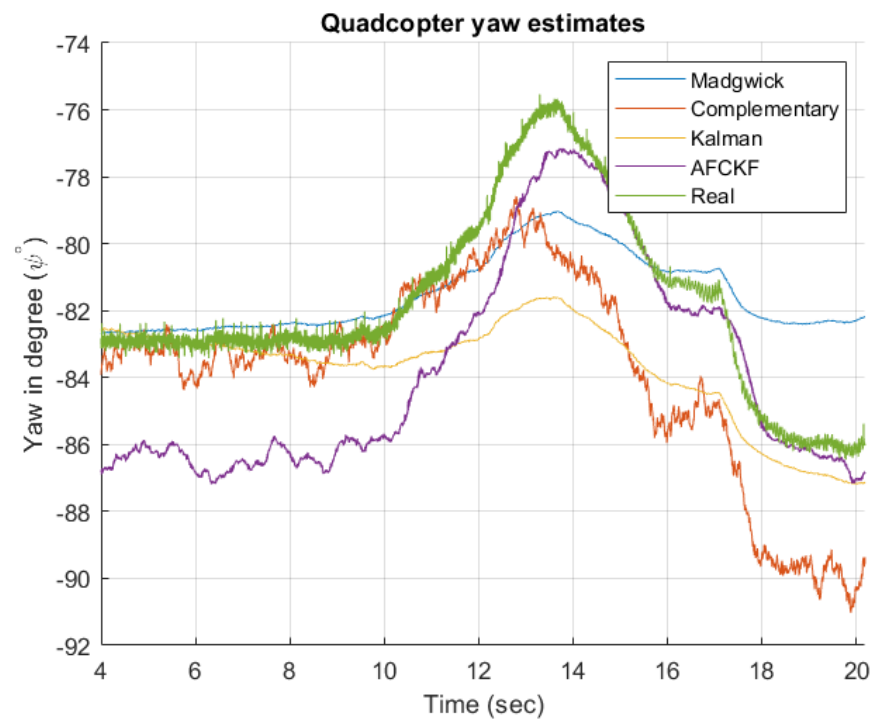

Figure A.4: Yaw estimates [Case: Hover without damaged rotor] 


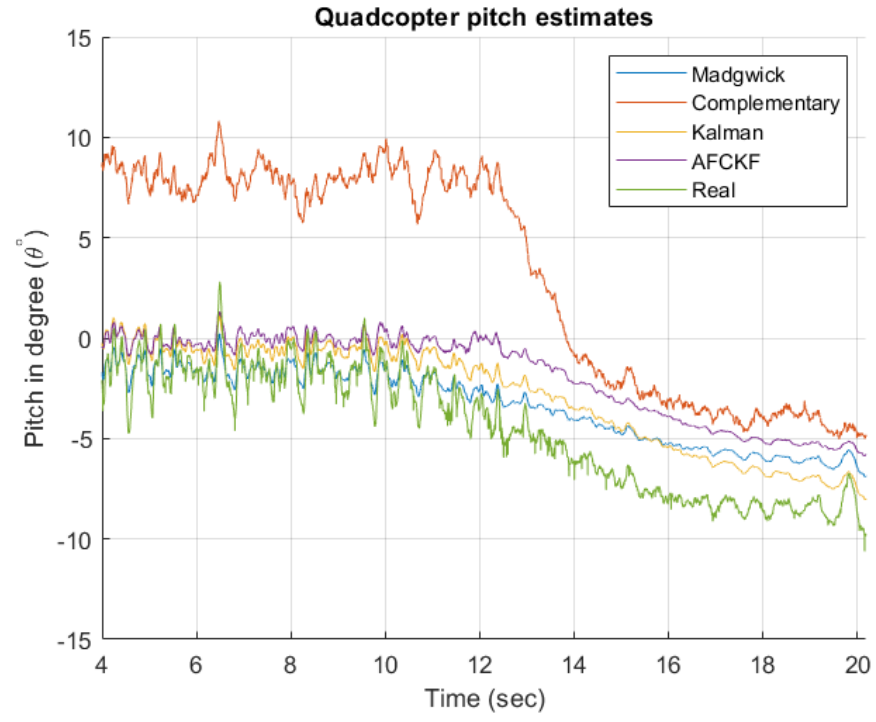

Figure A.5: Pitch estimates [Case: Hover without damaged rotor]

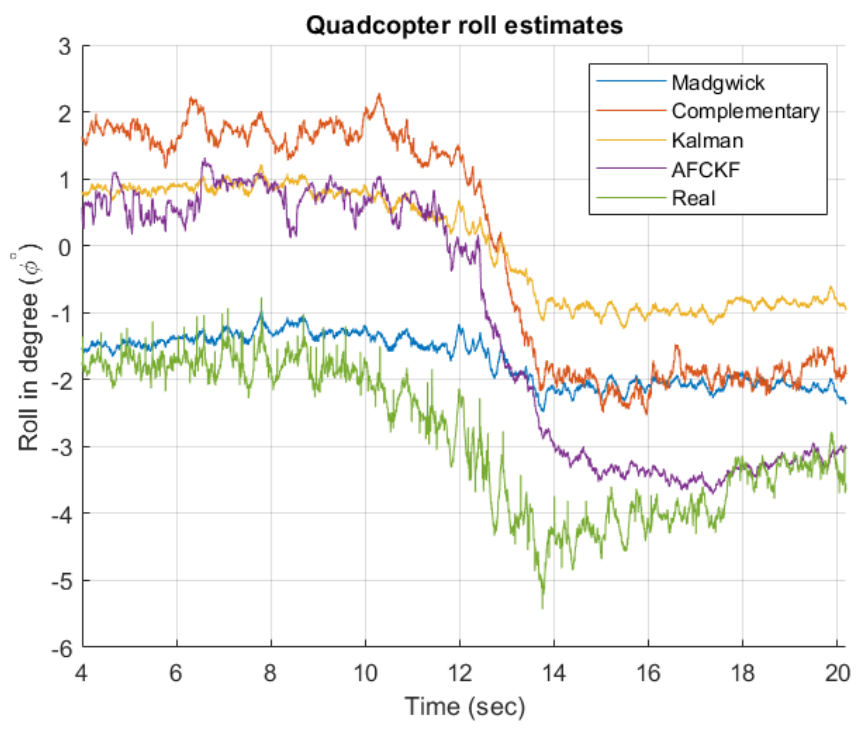

Figure A.6: Roll estimates [Case: Hover without damaged rotor] 

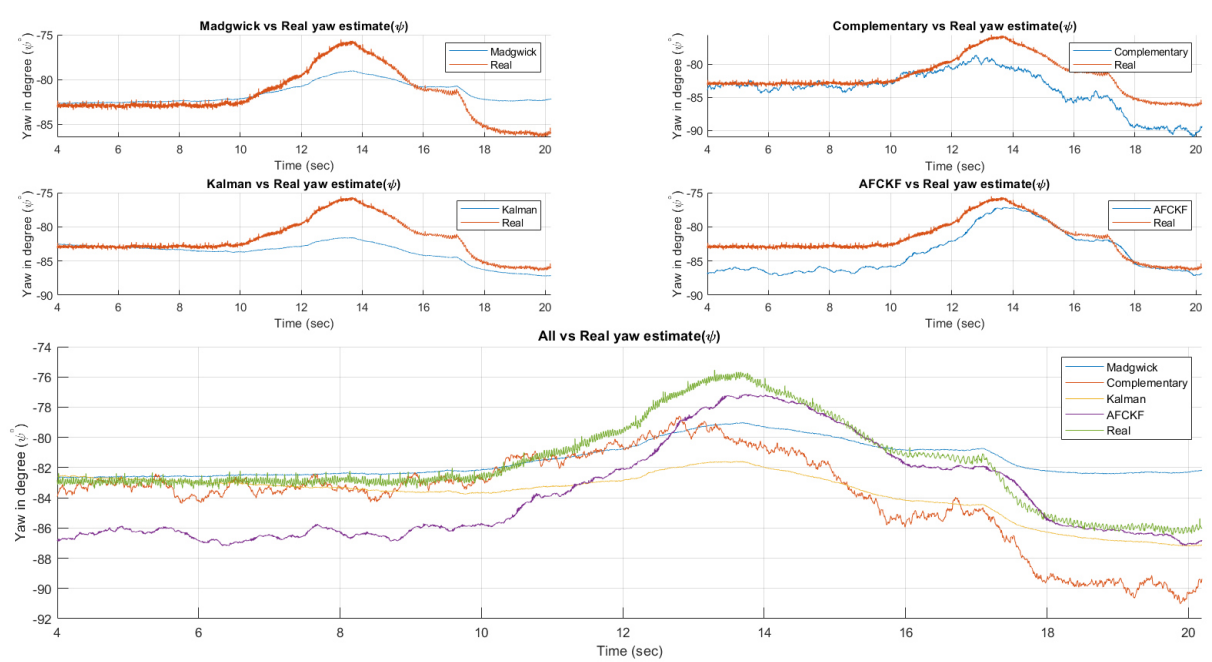

Figure A.7: Yaw estimates [Case: Hover without damaged rotor]
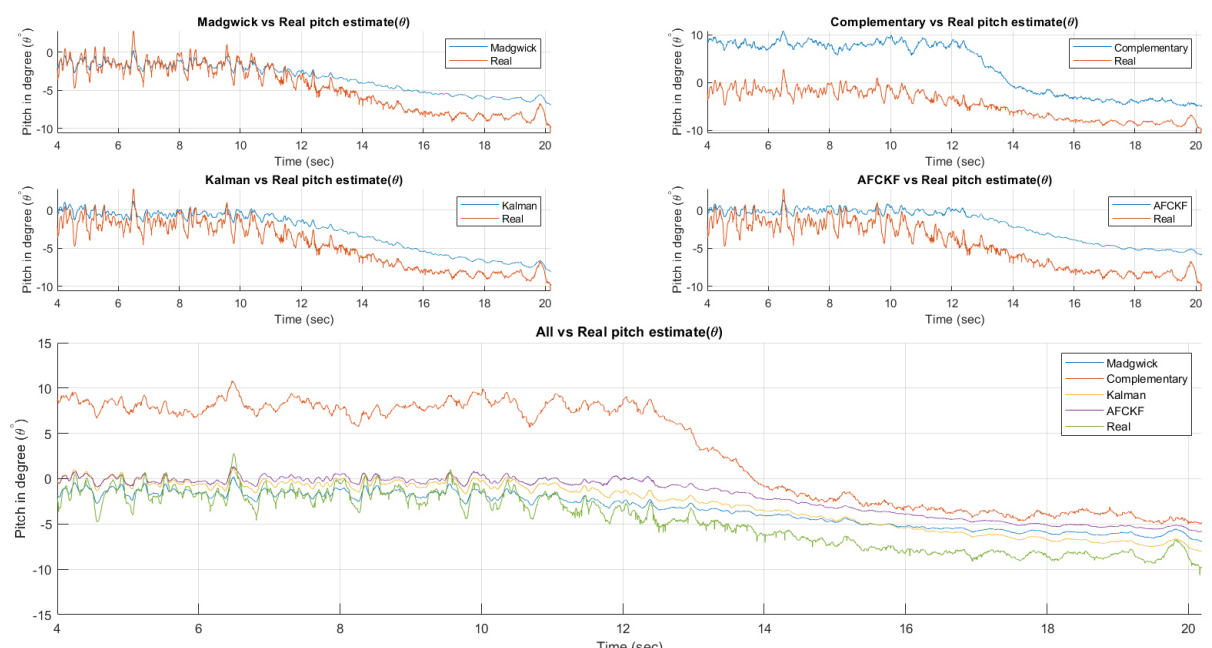

Figure A.8: Pitch estimates [Case: Hover without damaged rotor] 

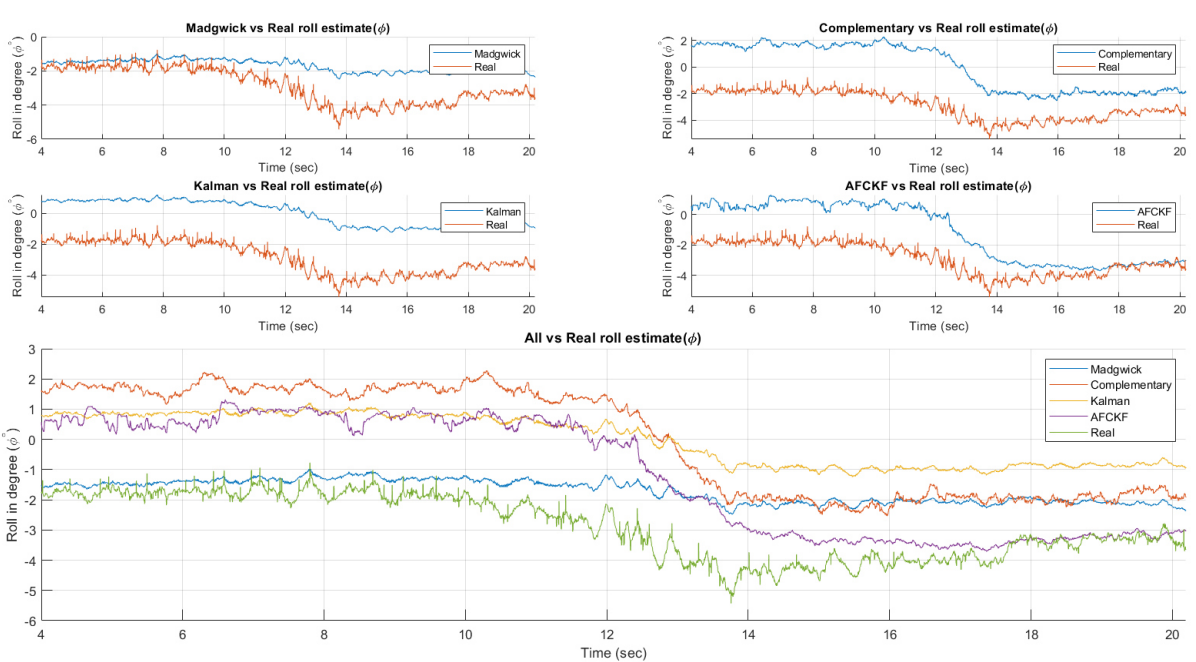

Figure A.9: Roll estimates [Case: Hover without damaged rotor]
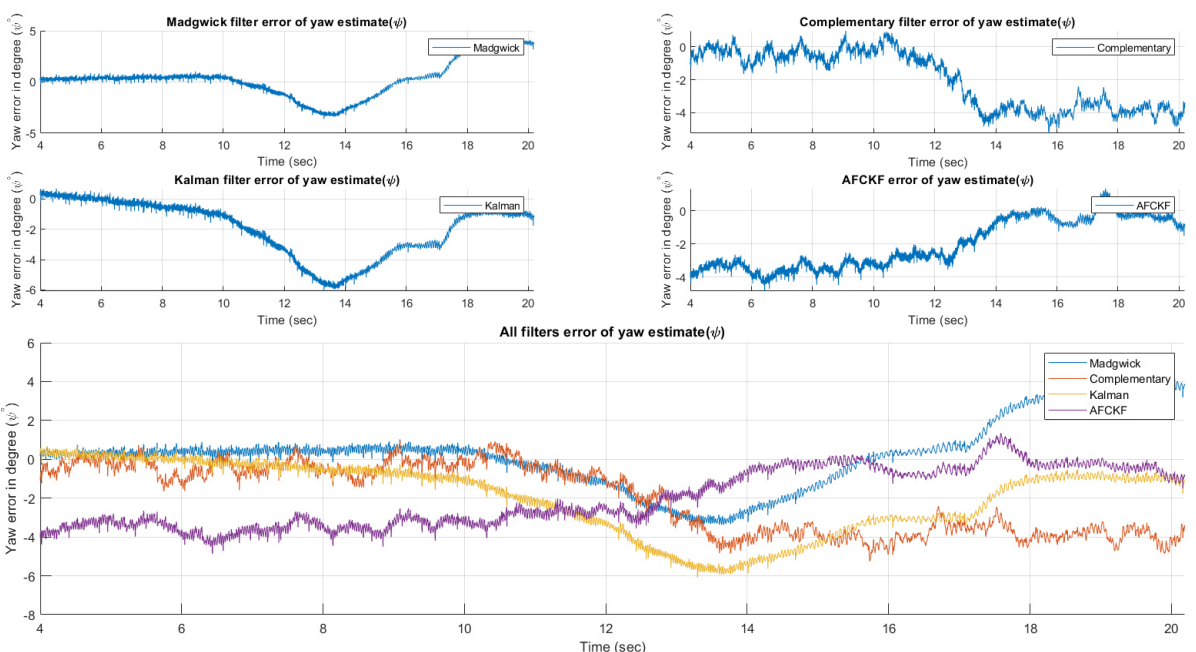

Figure A.10: Yaw estimate error of different filters [Case: Hover without damaged rotor] 


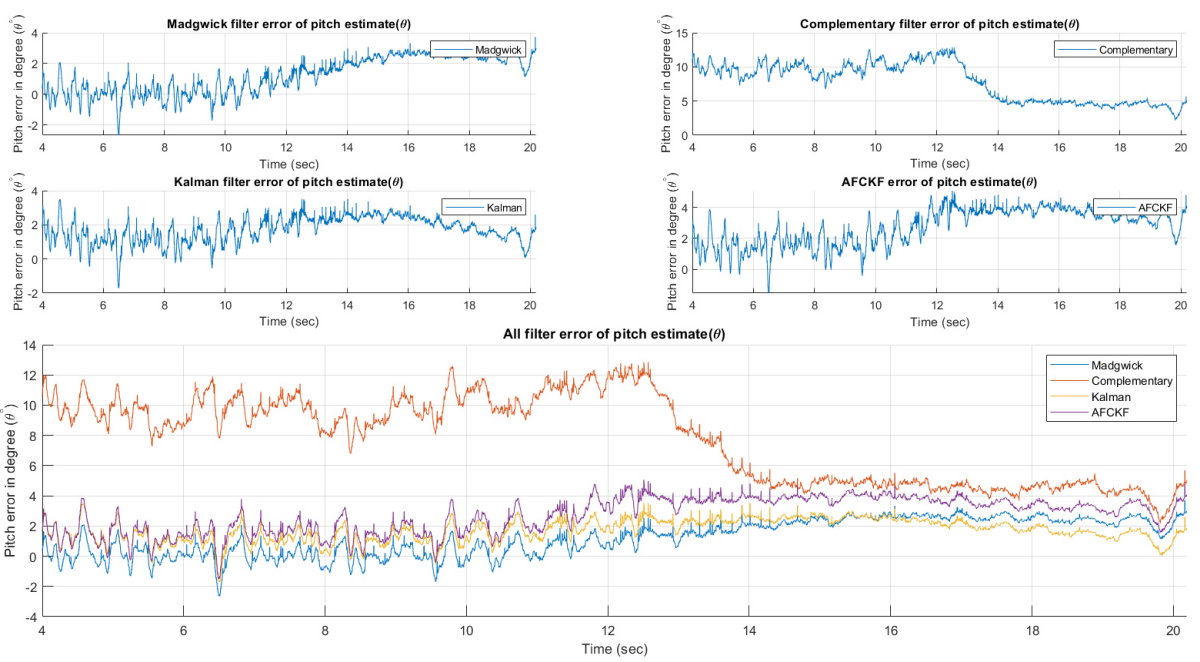

Figure A.11: Pitch estimate error of different filters [Case: Hover without damaged rotor]
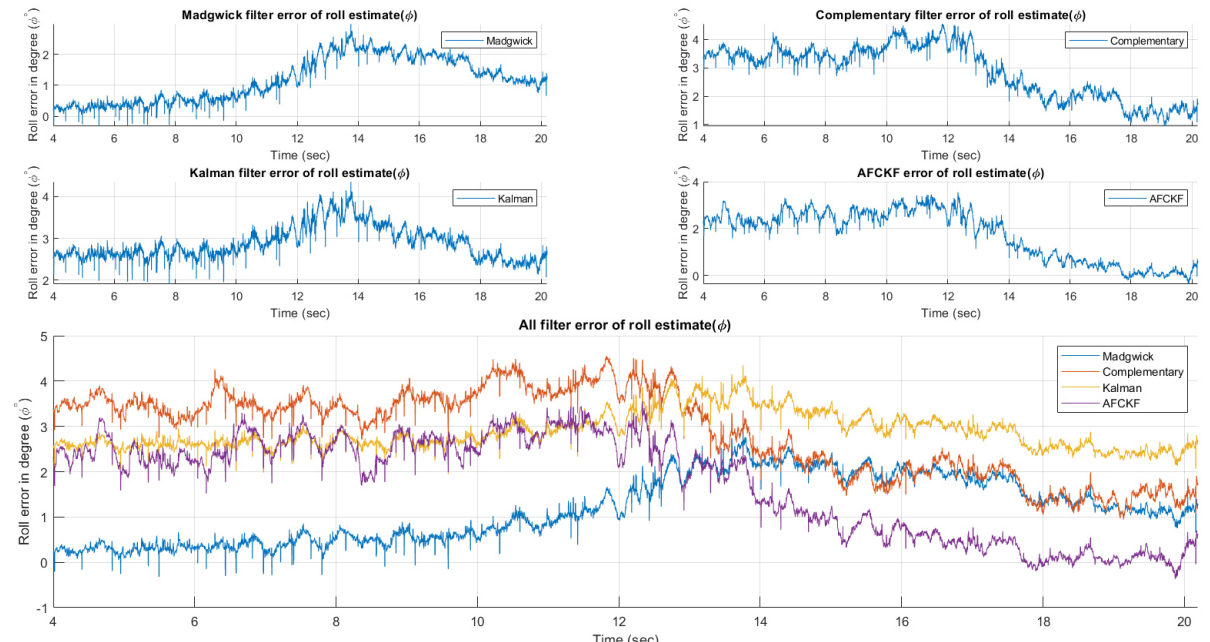

Figure A.12: Roll estimate error of different filters [Case: Hover without damaged rotor] 


\section{A.2 All results for the case of hover flight with damaged rotor}

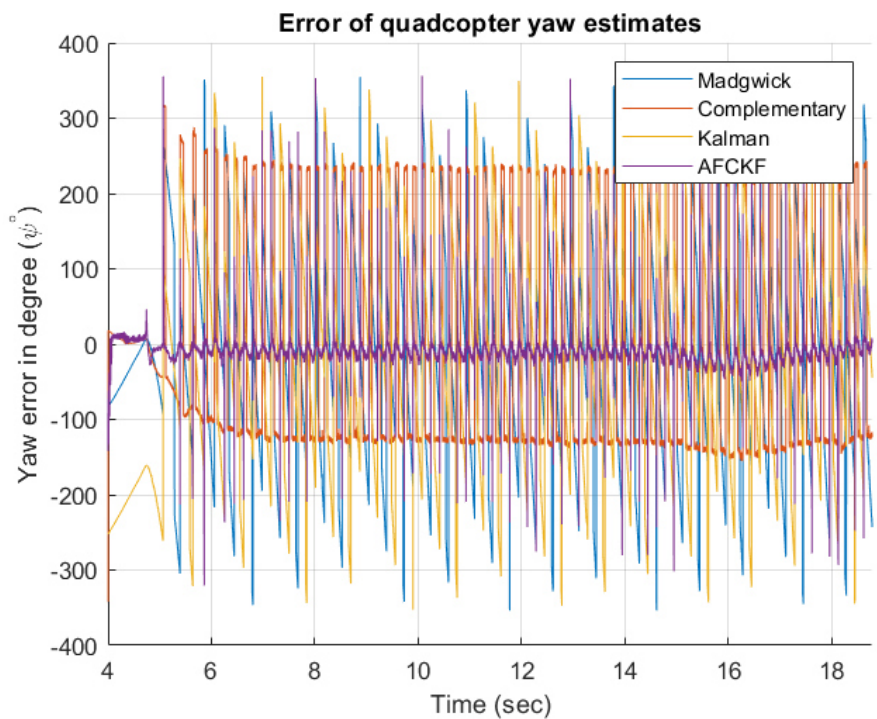

Figure A.13: Yaw estimate error of different filters [Case: Hover with damaged rotor]

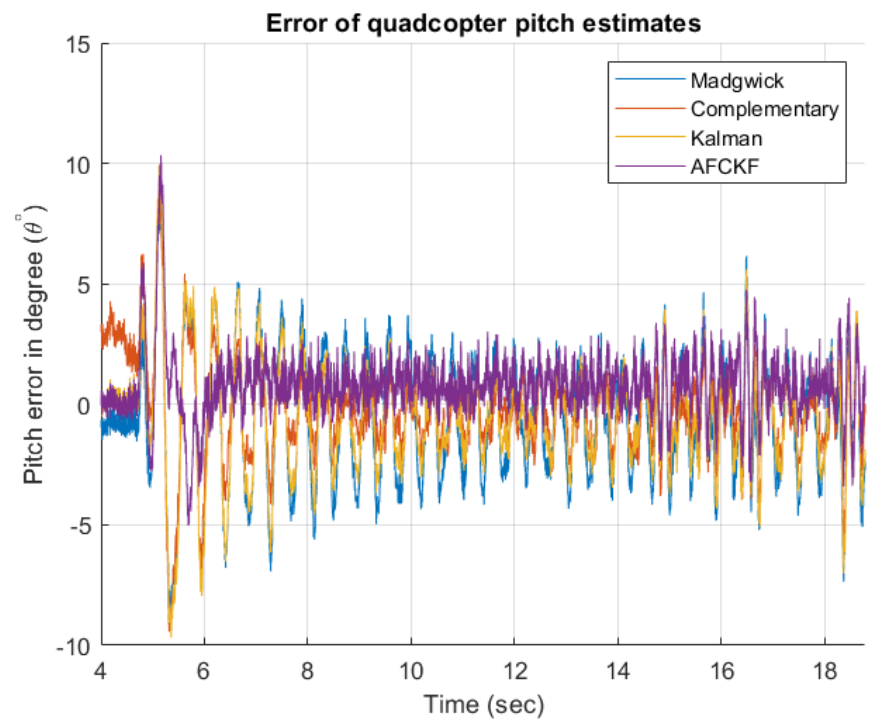

Figure A.14: Pitch estimate error of different filters [Case: Hover with damaged rotor] 


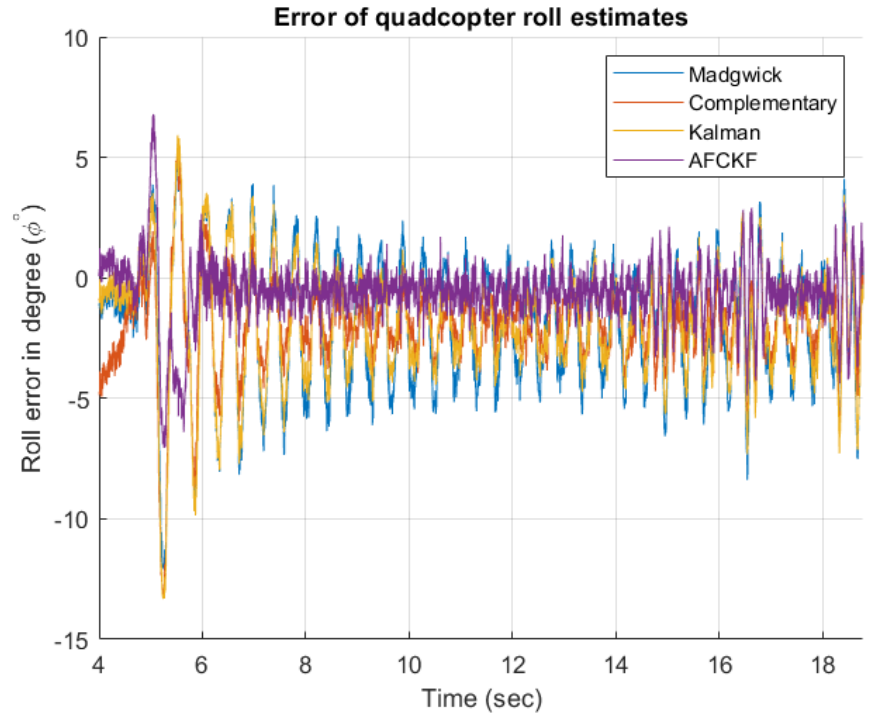

Figure A.15: Roll estimate error of different filters [Case: Hover with damaged rotor]

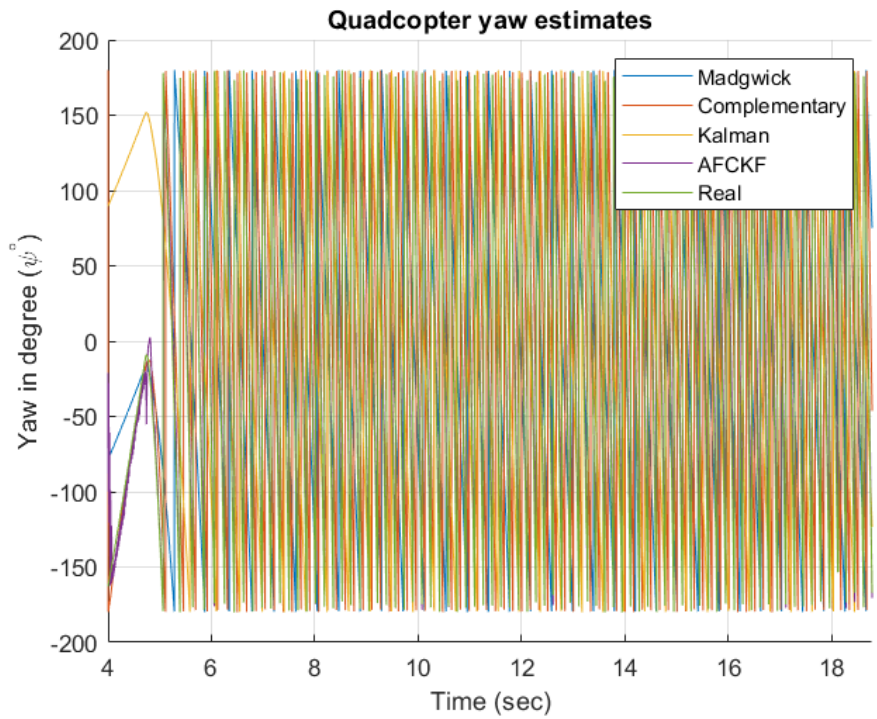

Figure A.16: Yaw estimates [Case: Hover with damaged rotor] 


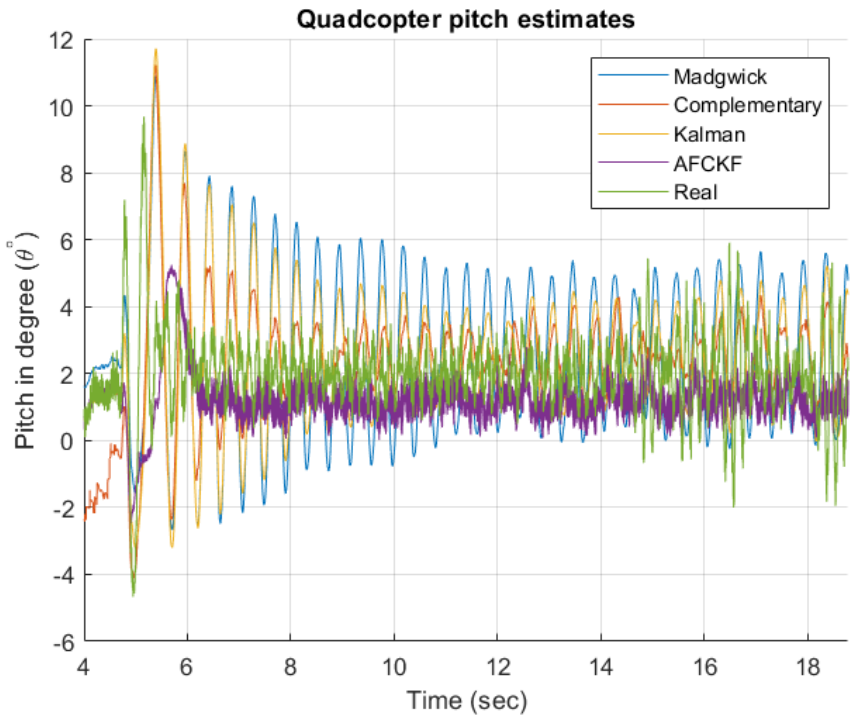

Figure A.17: Pitch estimates [Case: Hover with damaged rotor]

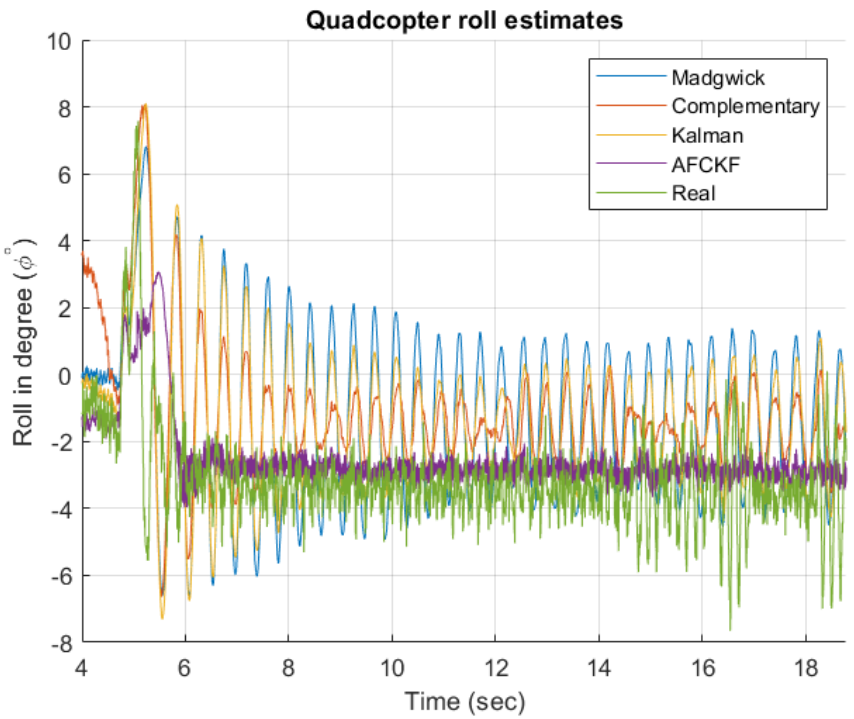

Figure A.18: Roll estimates [Case: Hover with damaged rotor] 


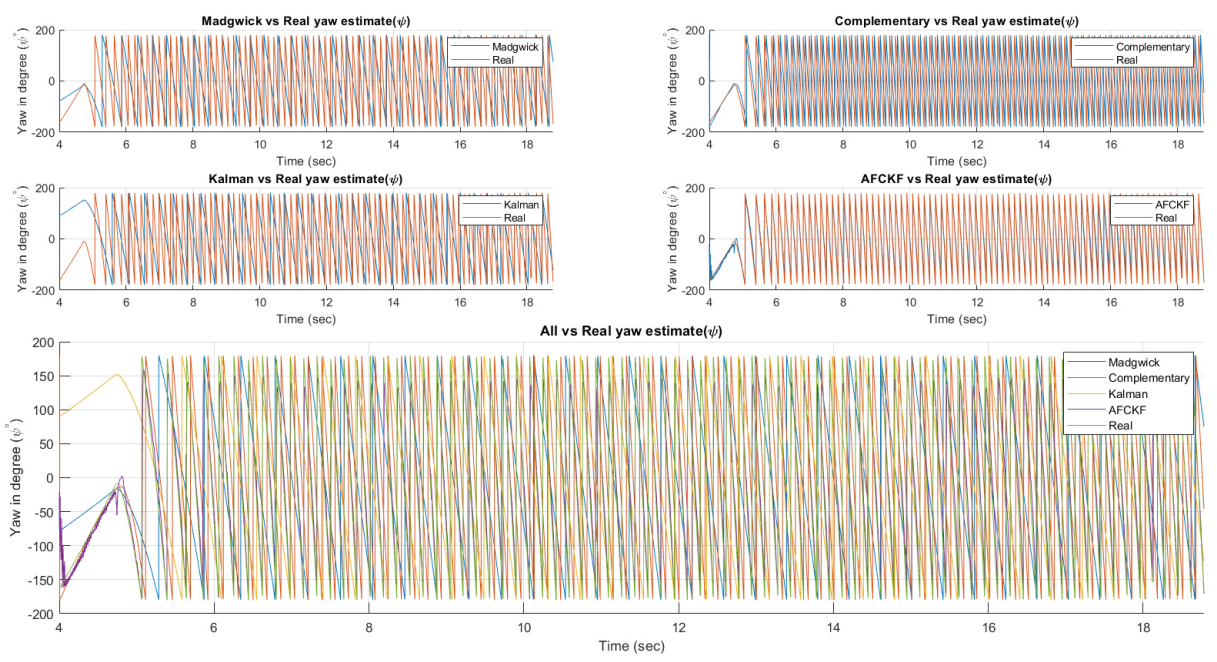

Figure A.19: Yaw estimates [Case: Hover with damaged rotor]
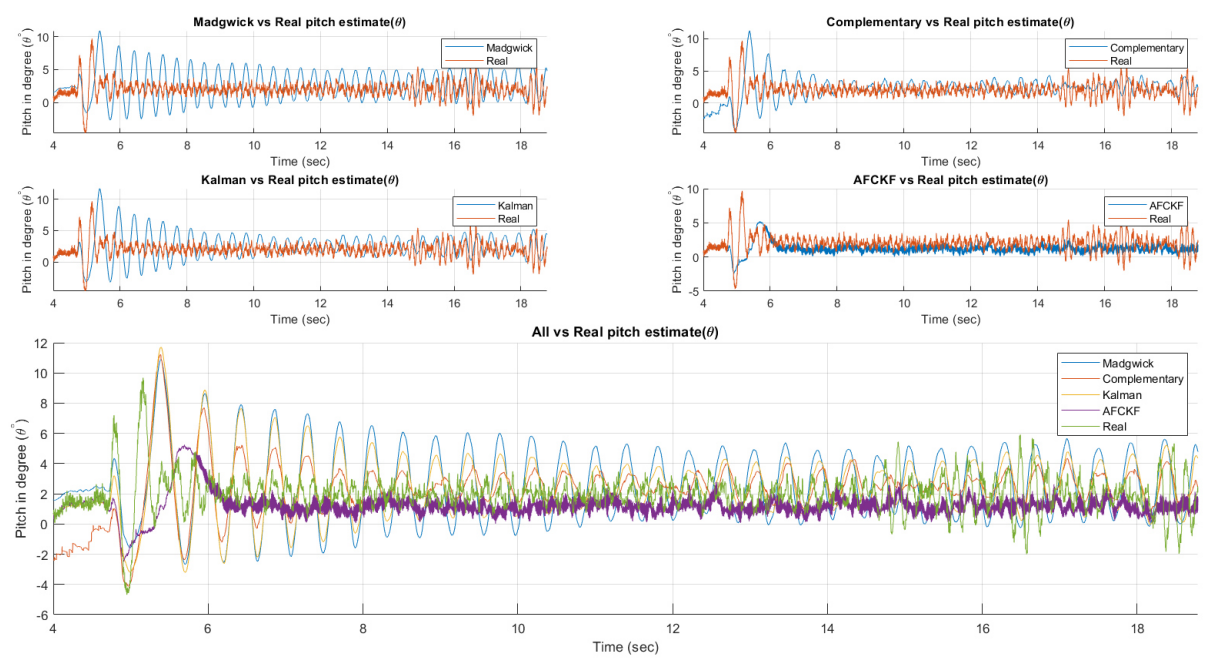

Figure A.20: Pitch estimates [Case: Hover with damaged rotor] 

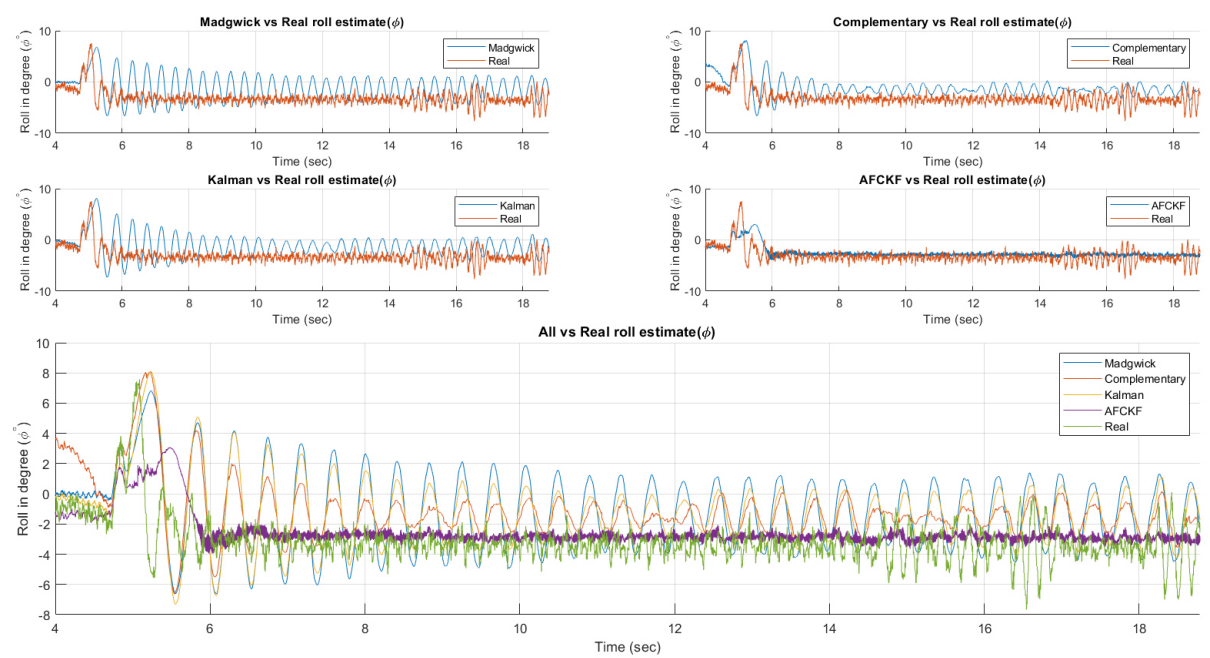

Figure A.21: Roll estimates [Case: Hover with damaged rotor]

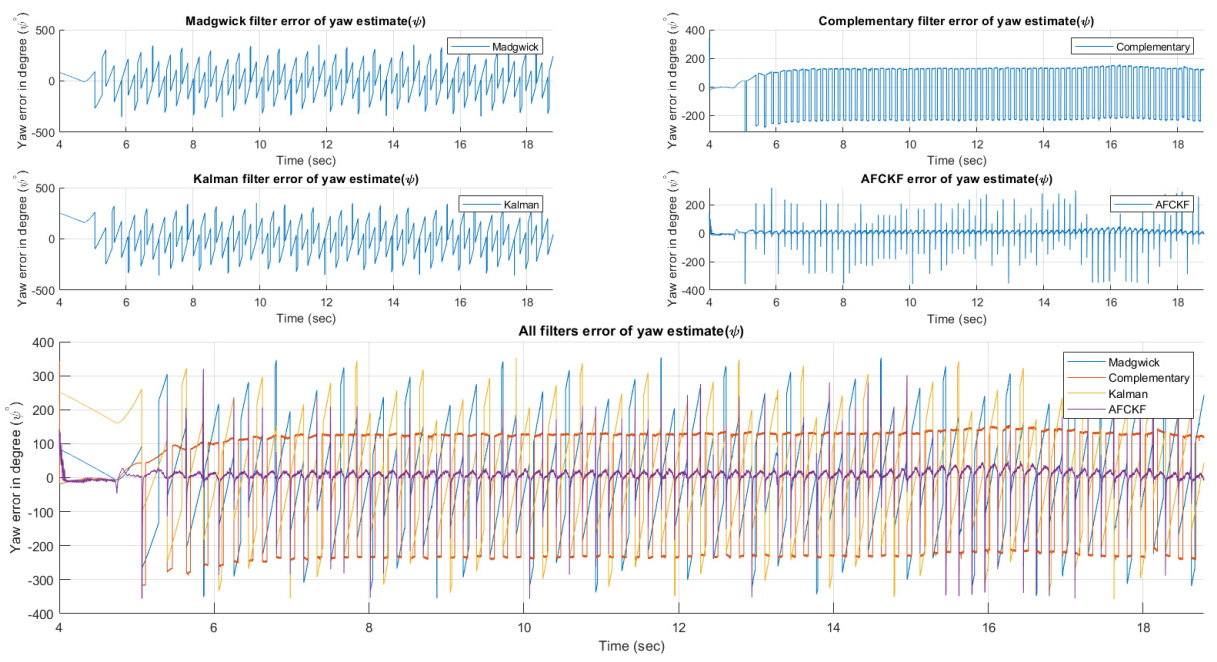

Figure A.22: Yaw estimate error of different filters [Case: Hover with damaged rotor] 

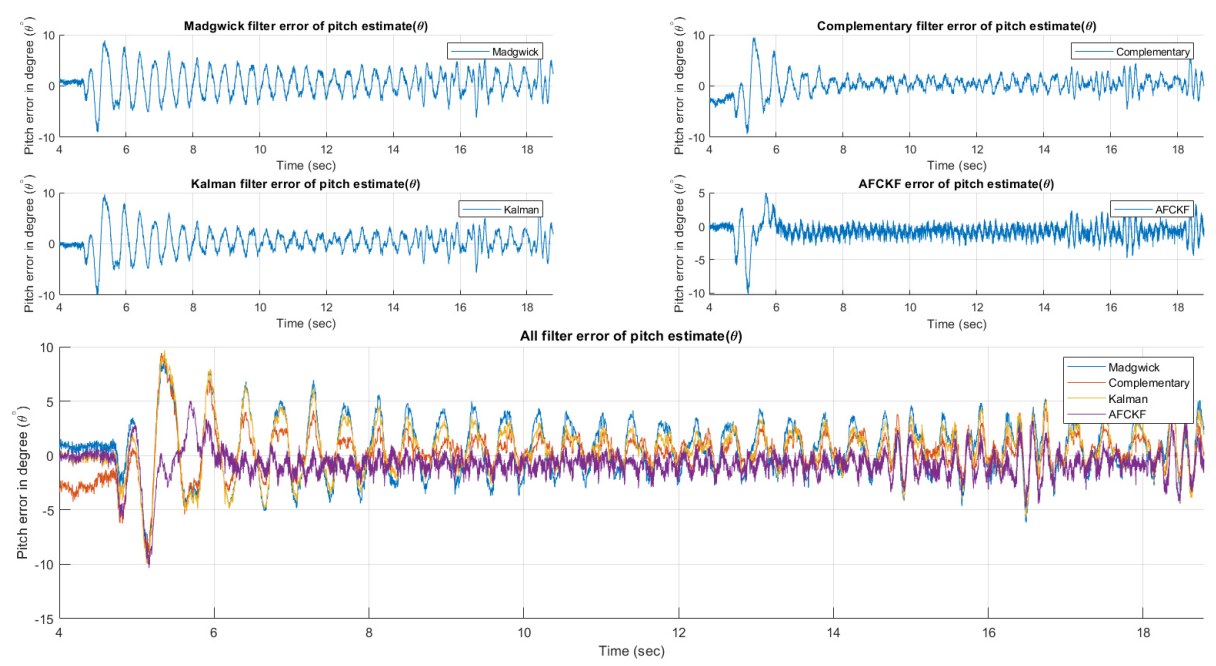

Figure A.23: Pitch estimate error of different filters [Case: Hover with damaged rotor]
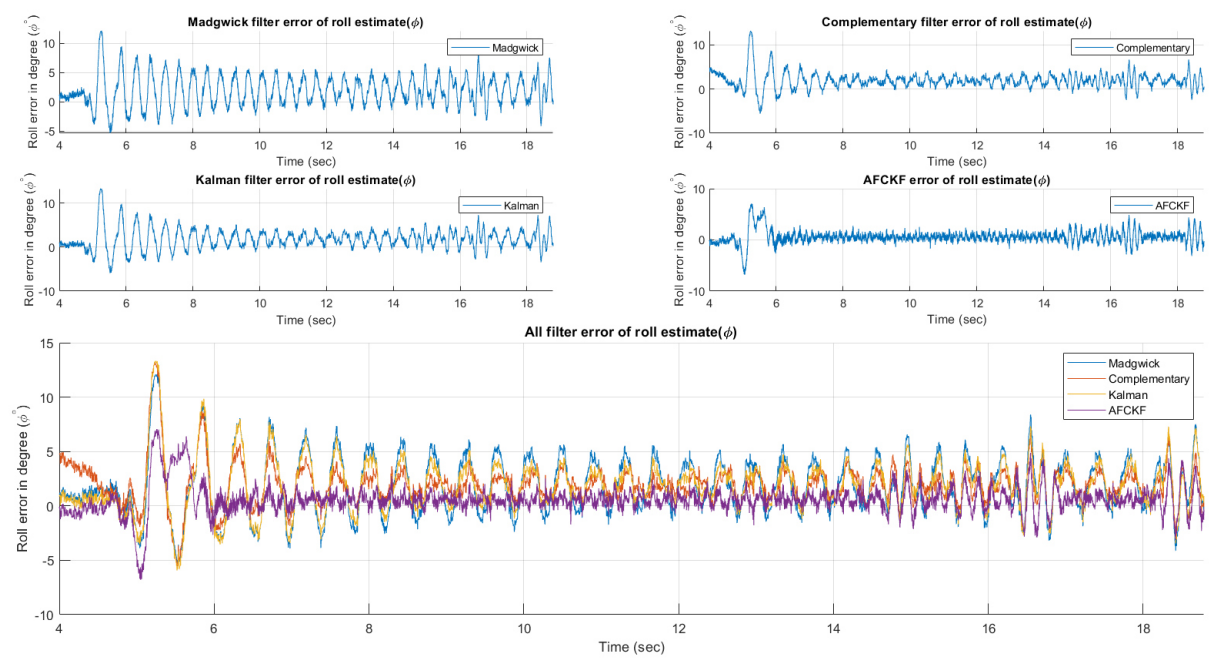

Figure A.24: Roll estimate error of different filters [Case: Hover with damaged rotor] 


\section{A.3 All results for the case of aggressive flight without damaged rotor}

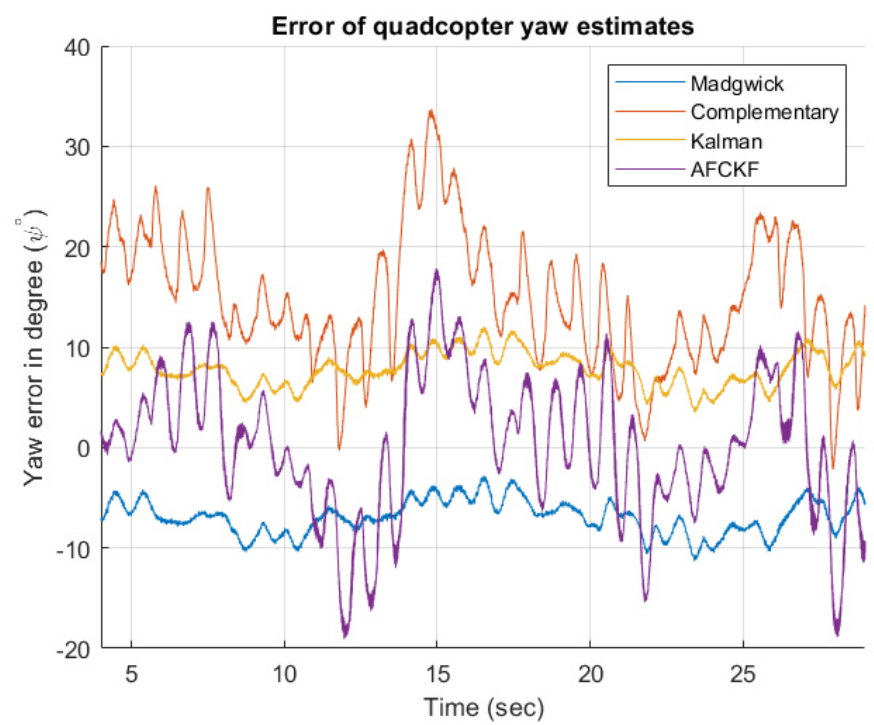

Figure A.25: Yaw estimate error of different filters [Case: Aggressive motion without damaged rotor]

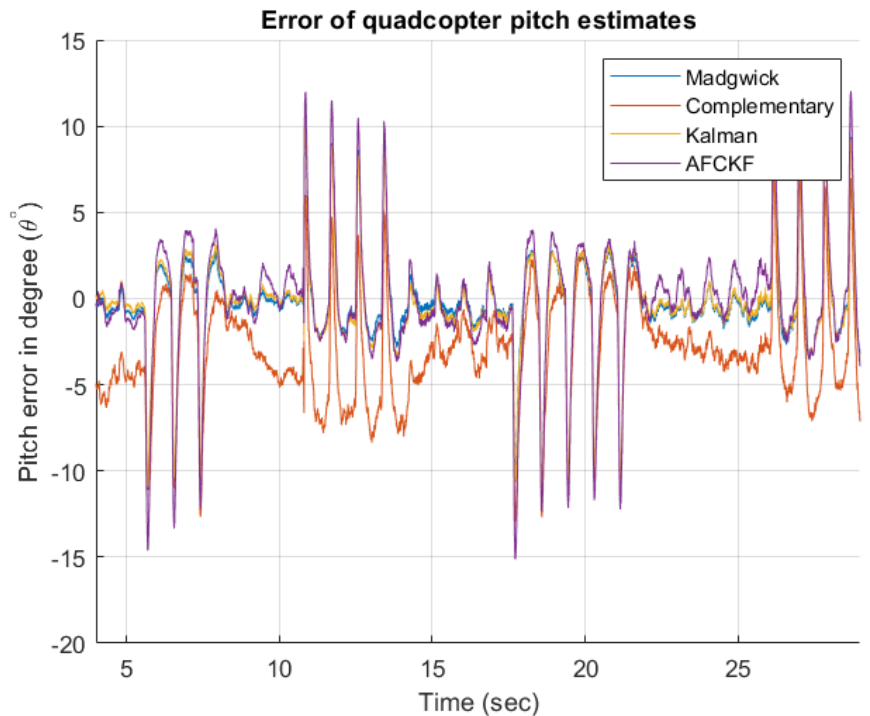

Figure A.26: Pitch estimate error of different filters [Case: Aggressive motion without damaged rotor] 


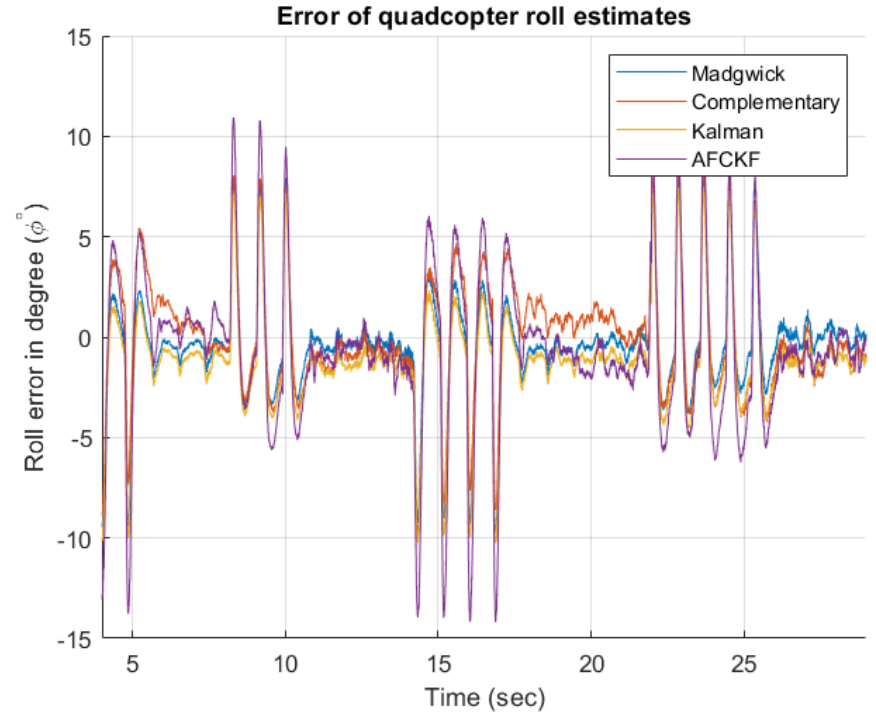

Figure A.27: Roll estimate error of different filters [Case: Aggressive motion without damaged rotor]

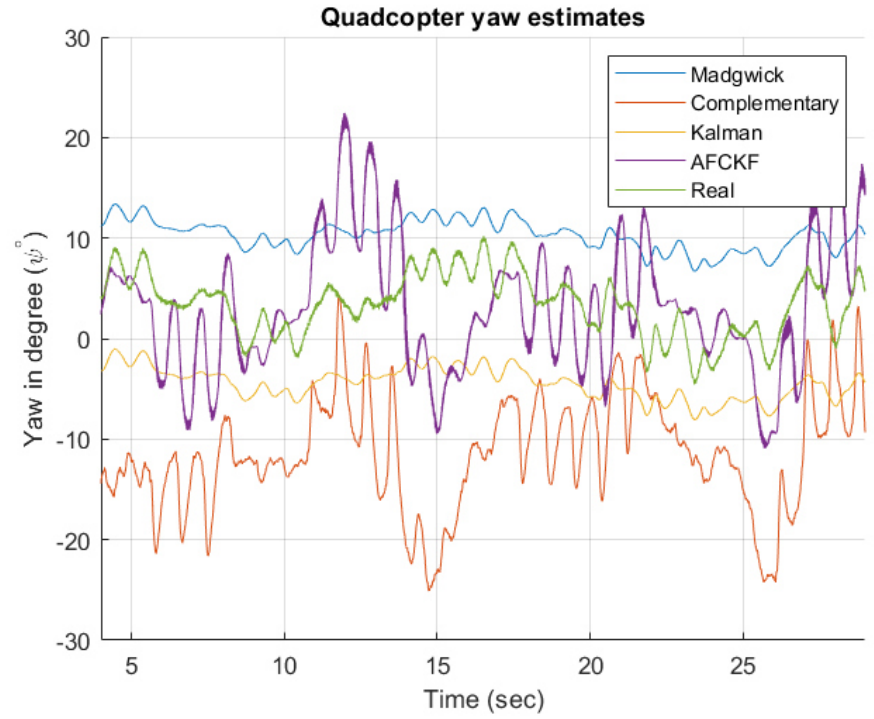

Figure A.28: Yaw estimates [Case: Aggressive motion without damaged rotor] 


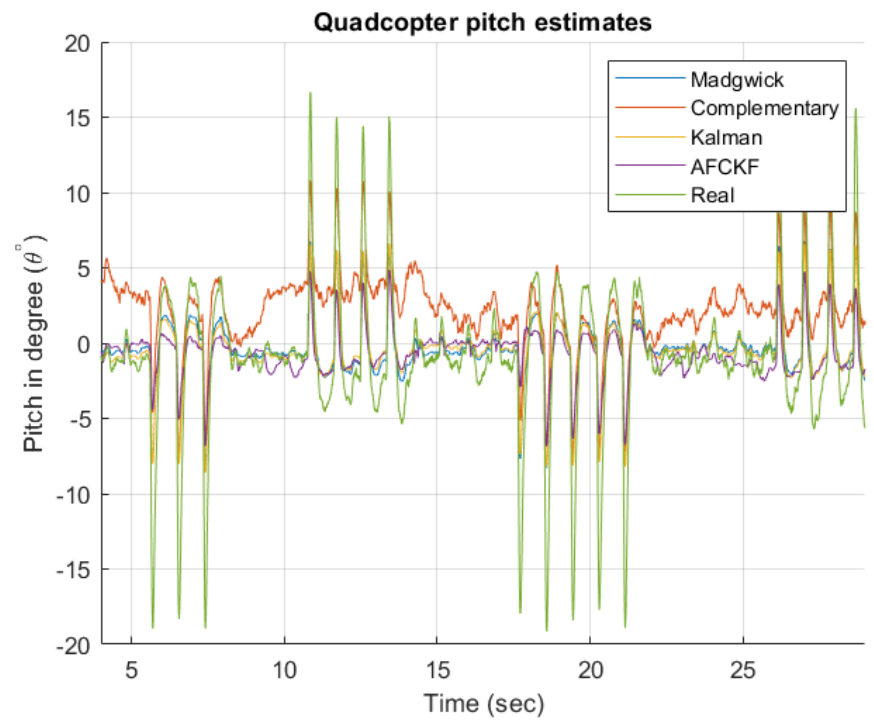

Figure A.29: Pitch estimates [Case: Aggressive motion without damaged rotor]

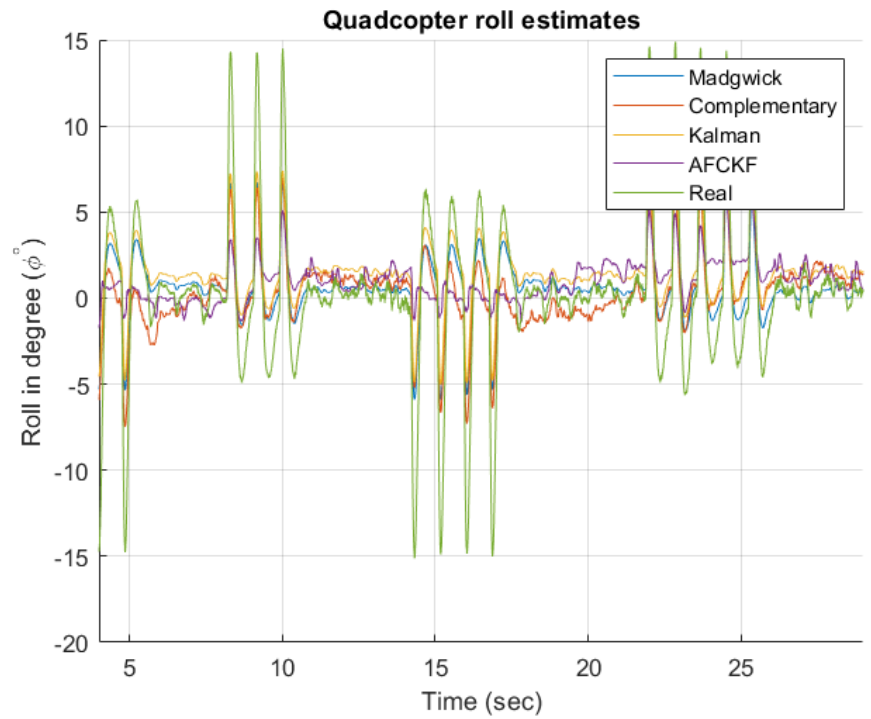

Figure A.30: Roll estimates [Case: Aggressive motion without damaged rotor] 

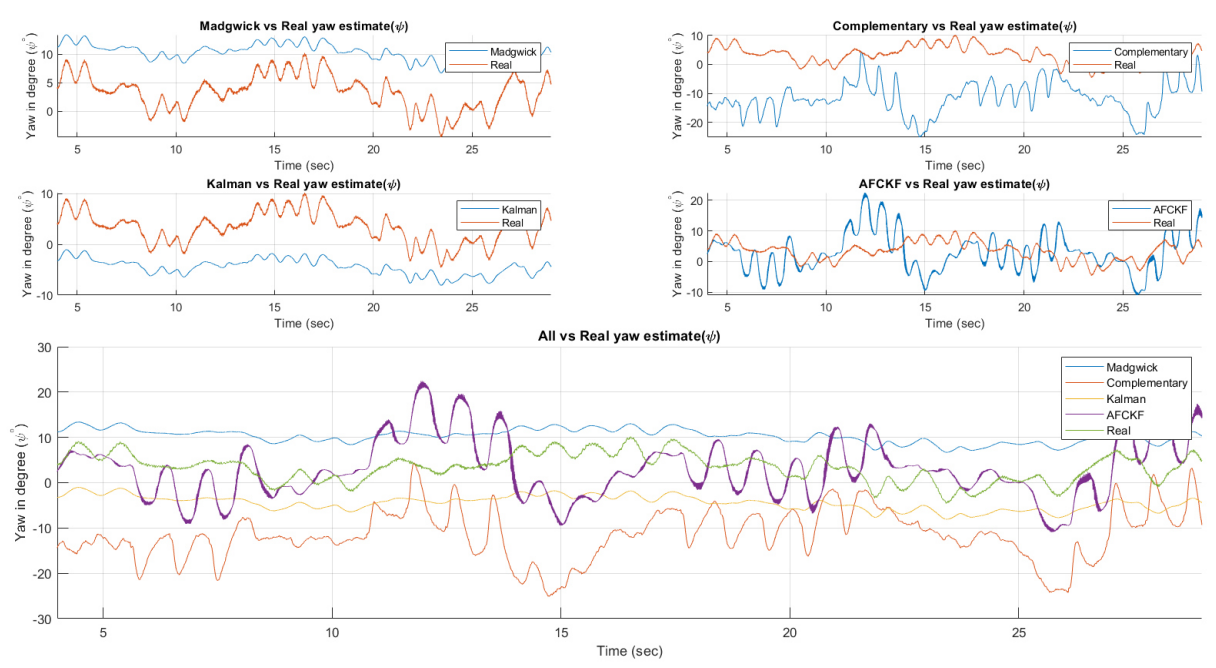

Figure A.31: Yaw estimates [Case: Aggressive motion without damaged rotor]
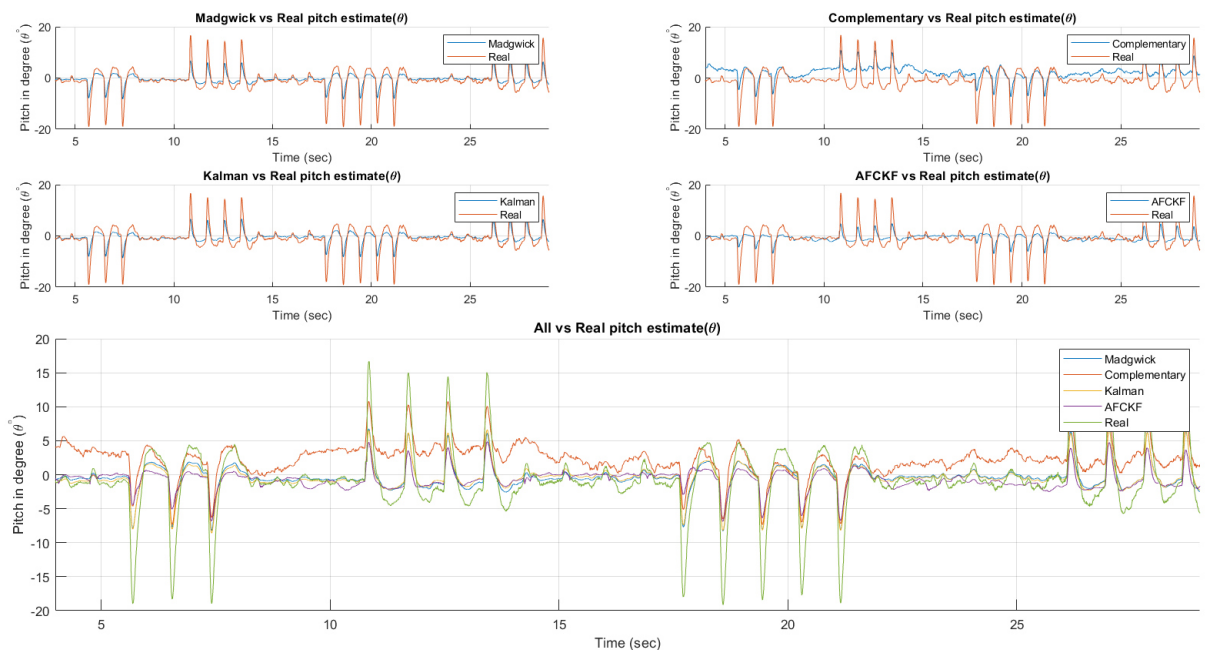

Figure A.32: Pitch estimates [Case: Aggressive motion without damaged rotor] 

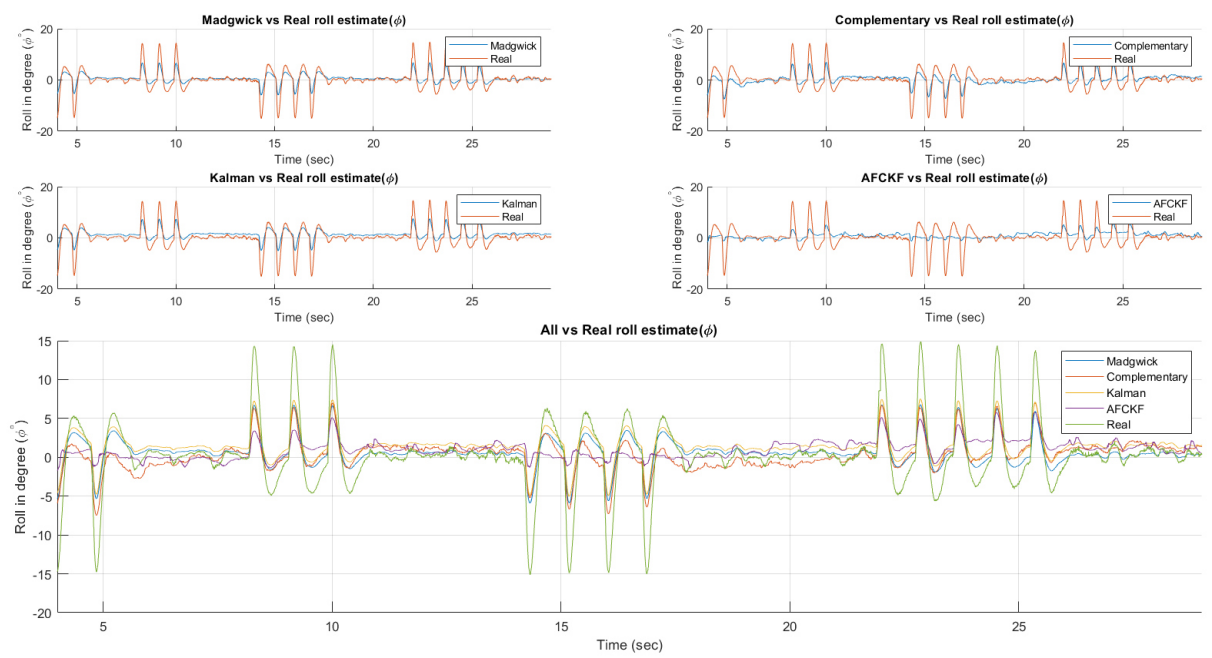

Figure A.33: Roll estimates [Case: Aggressive motion without damaged rotor]
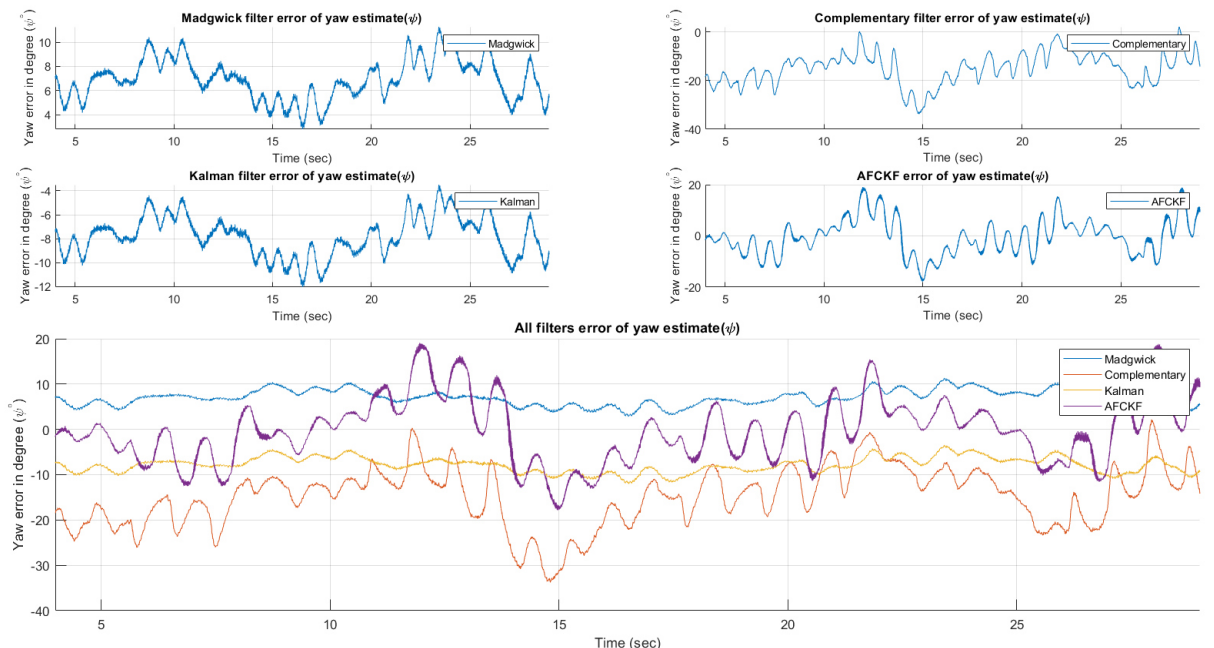

Figure A.34: Yaw estimate error of different filters [Case: Aggressive motion without damaged rotor] 

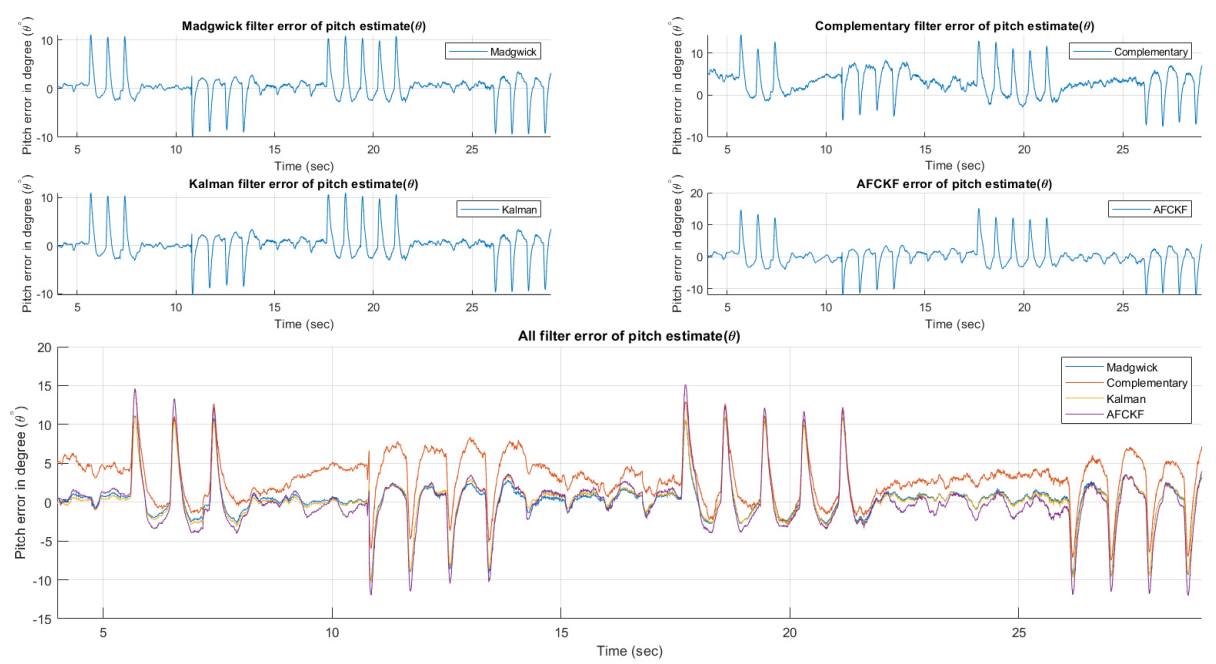

Figure A.35: Pitch estimate error of different filters [Case: Aggressive motion without damaged rotor]
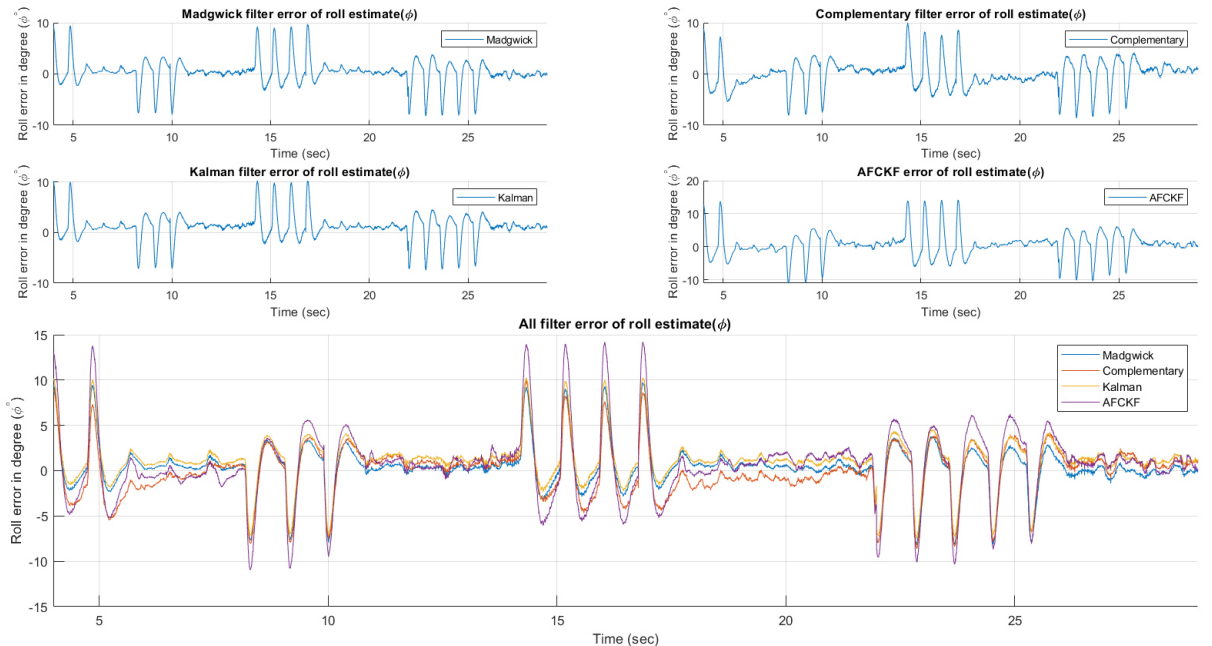

Figure A.36: Roll estimate error of different filters [Case: Aggressive motion without damaged rotor] 


\section{A.4 All results for the case of aggressive flight with damaged rotor}

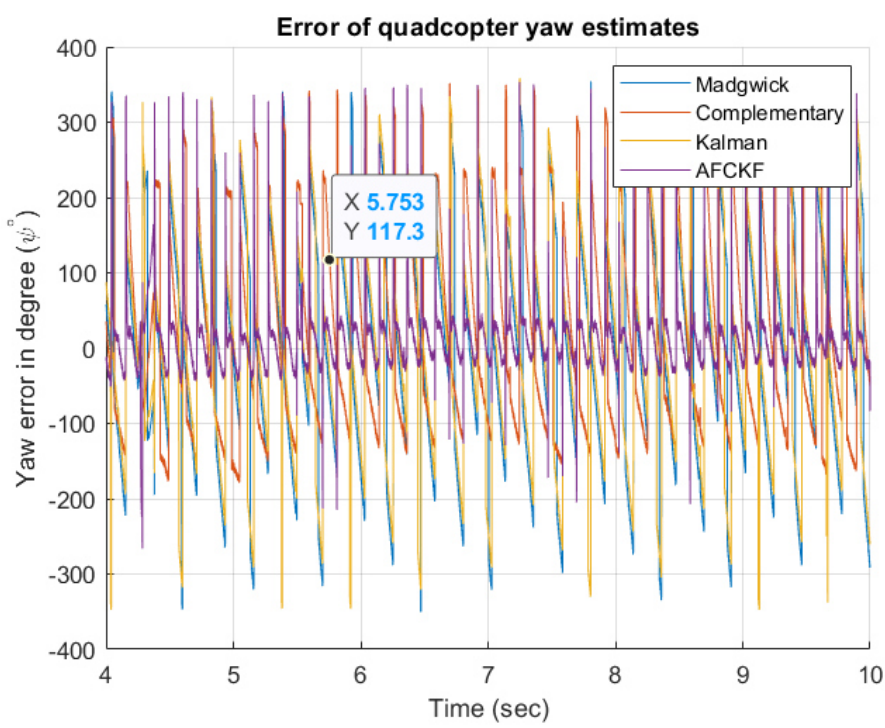

Figure A.37: Yaw estimate error of different filters [Case: Aggressive motion with damaged rotor]

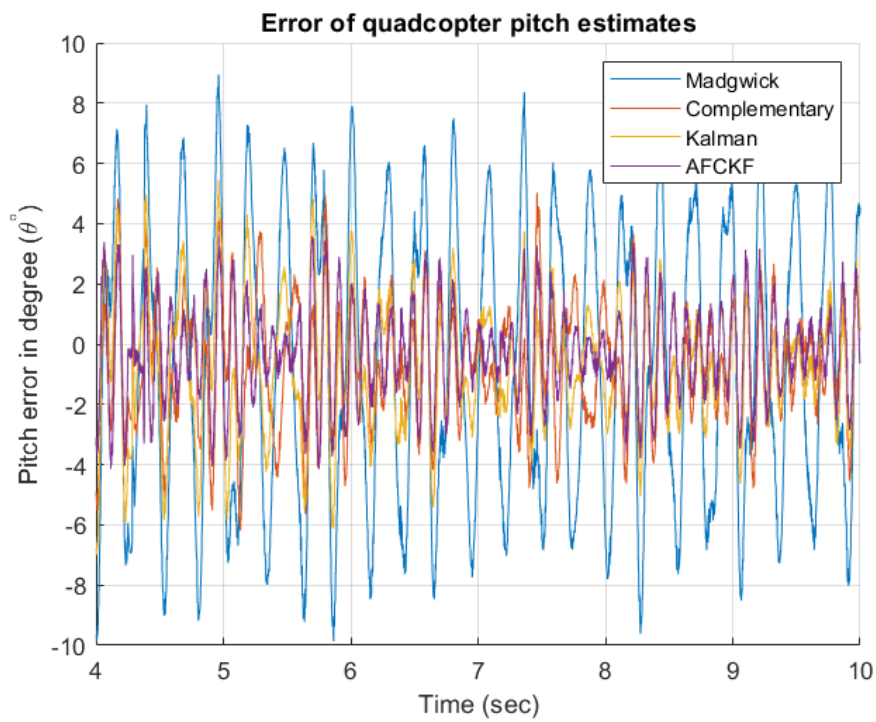

Figure A.38: Pitch estimate error of different filters [Case: Aggressive motion with damaged rotor] 


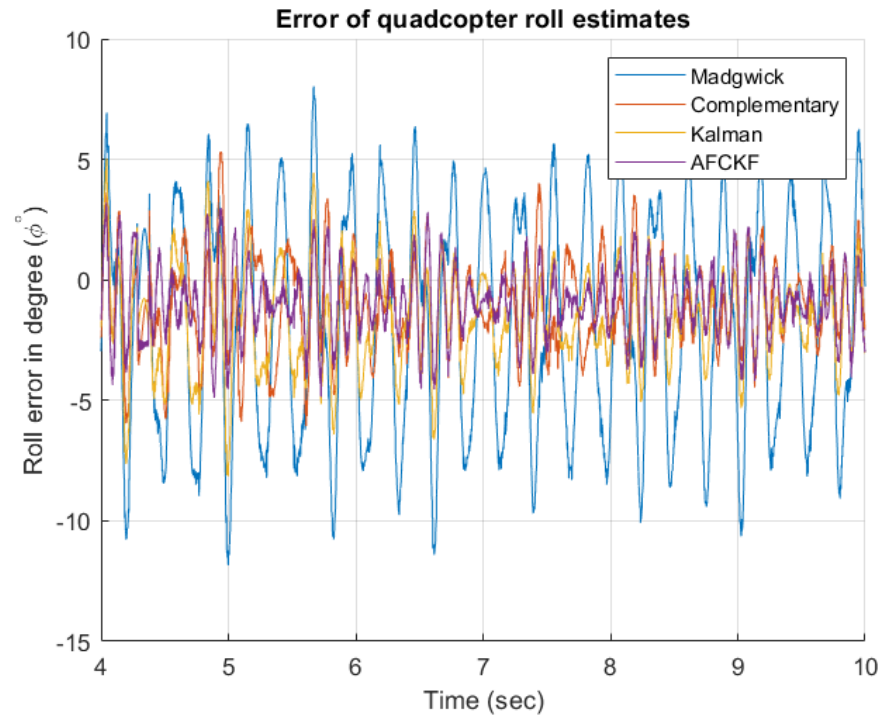

Figure A.39: Roll estimate error of different filters [Case: Aggressive motion with damaged rotor]

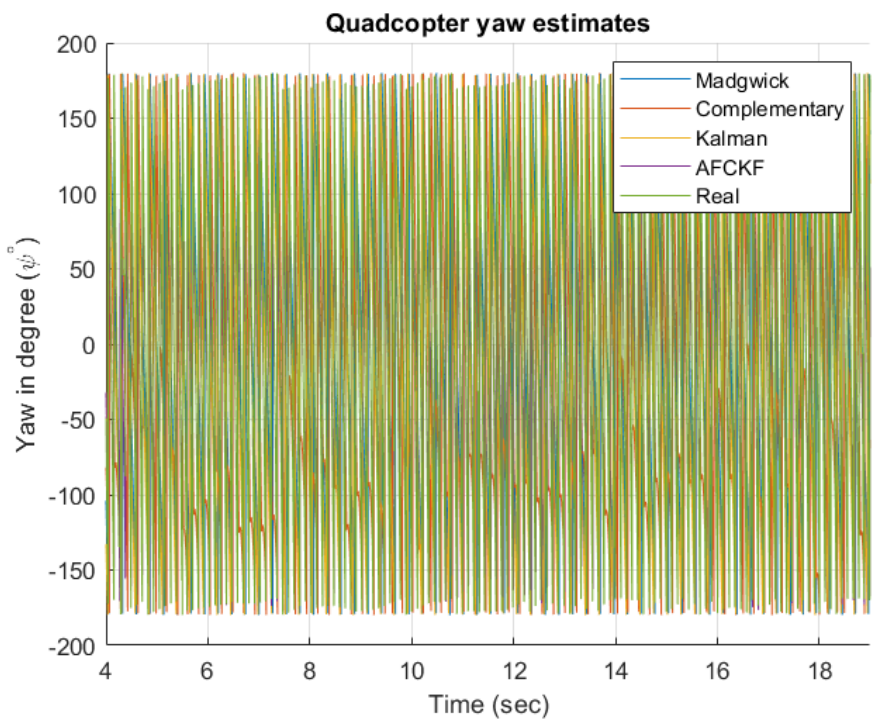

Figure A.40: Yaw estimates [Case: Aggressive motion with damaged rotor] 


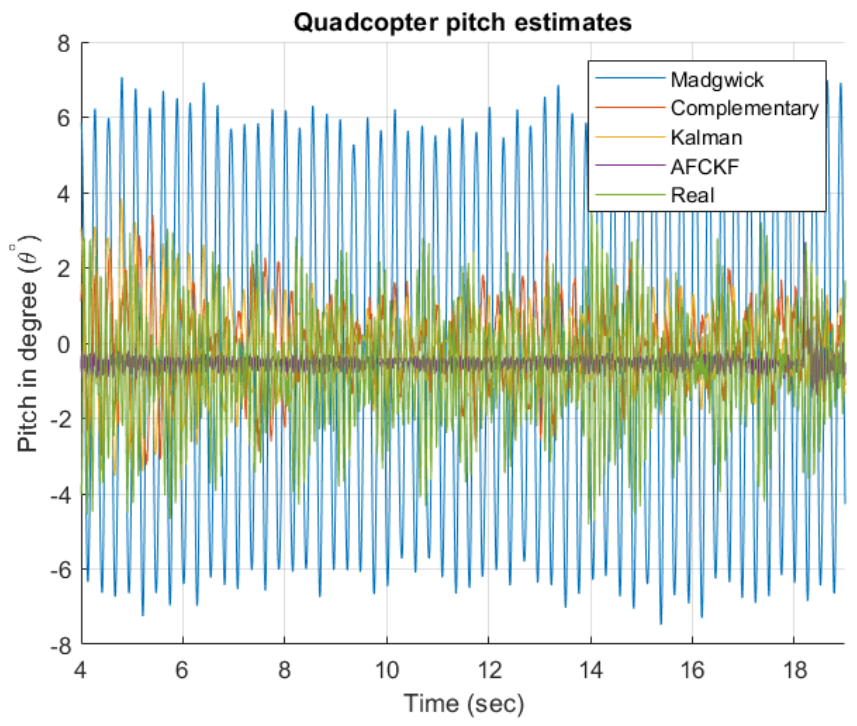

Figure A.41: Pitch estimates [Case: Aggressive motion with damaged rotor]

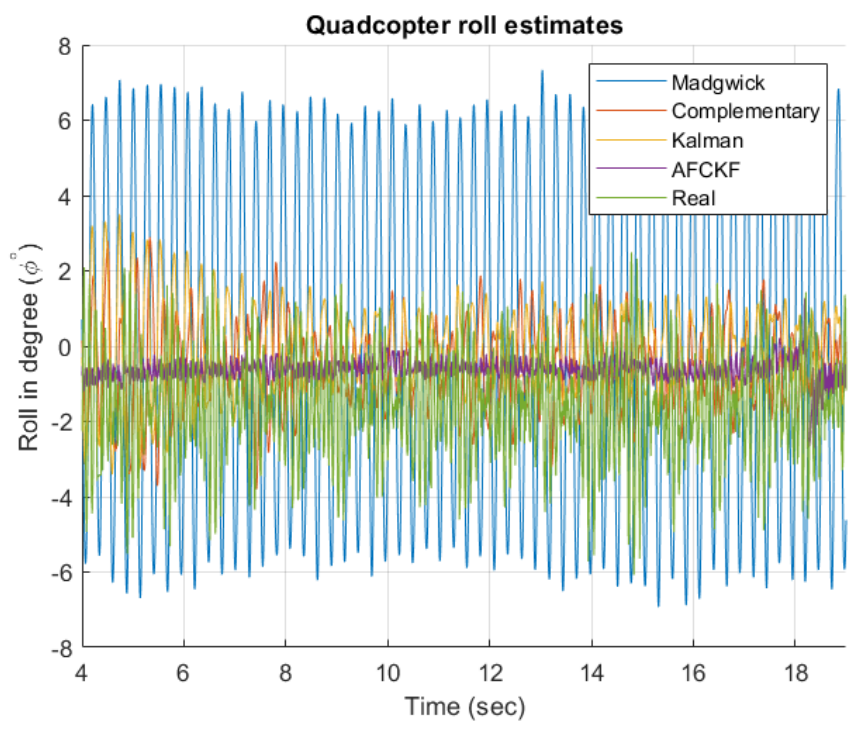

Figure A.42: Roll estimates [Case: Aggressive motion with damaged rotor] 

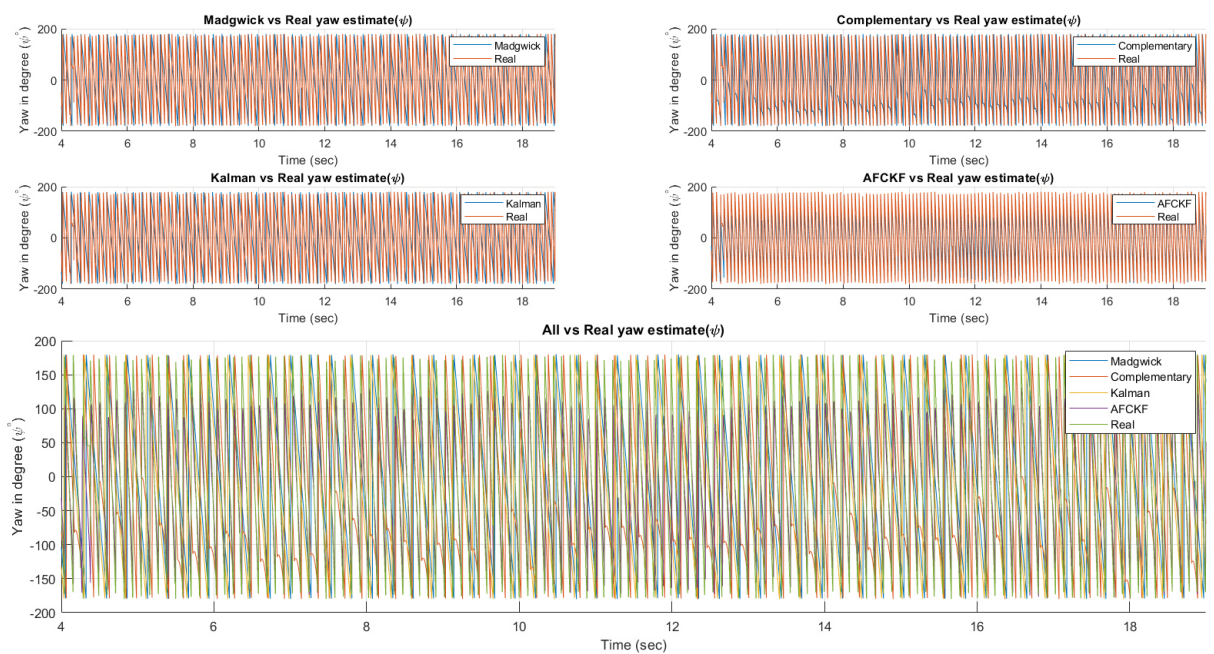

Figure A.43: Yaw estimates [Case: Aggressive motion with damaged rotor]
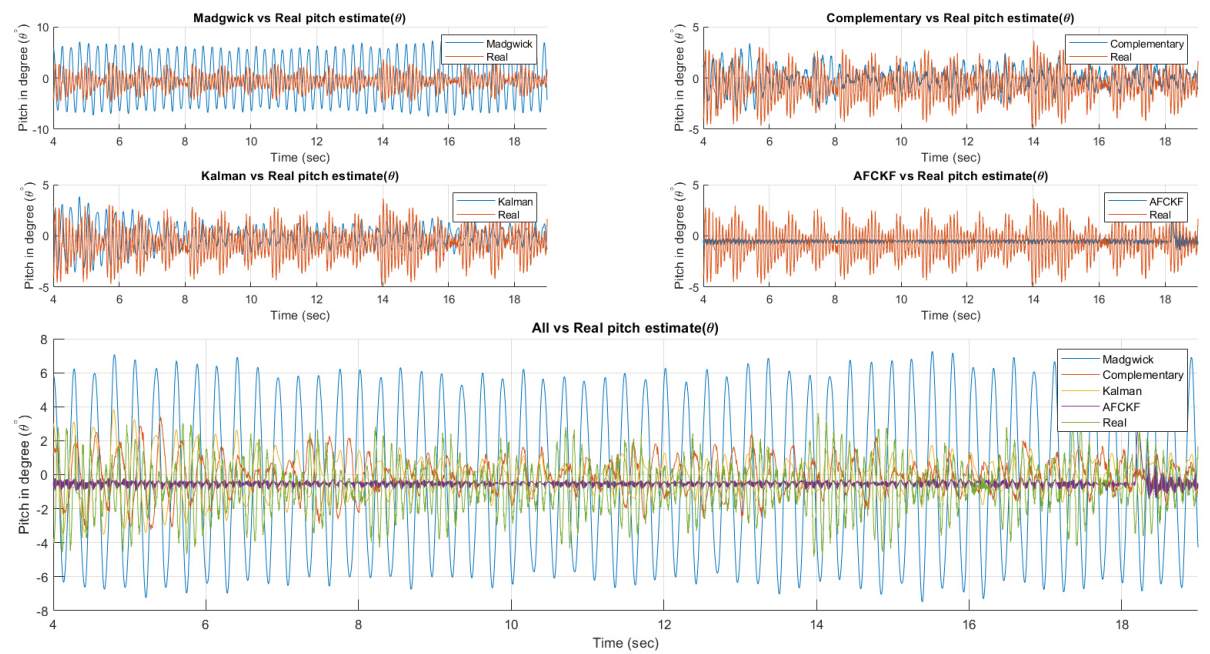

Figure A.44: Pitch estimates [Case: Aggressive motion with damaged rotor] 


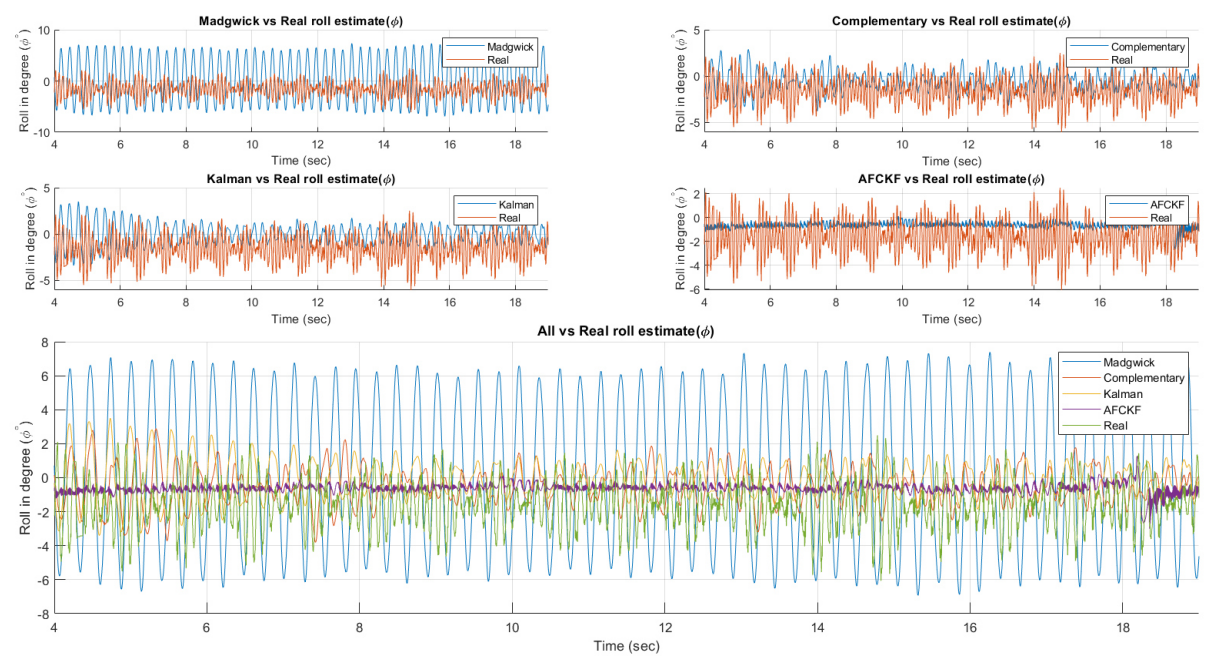

Figure A.45: Roll estimates [Case: Aggressive motion with damaged rotor]

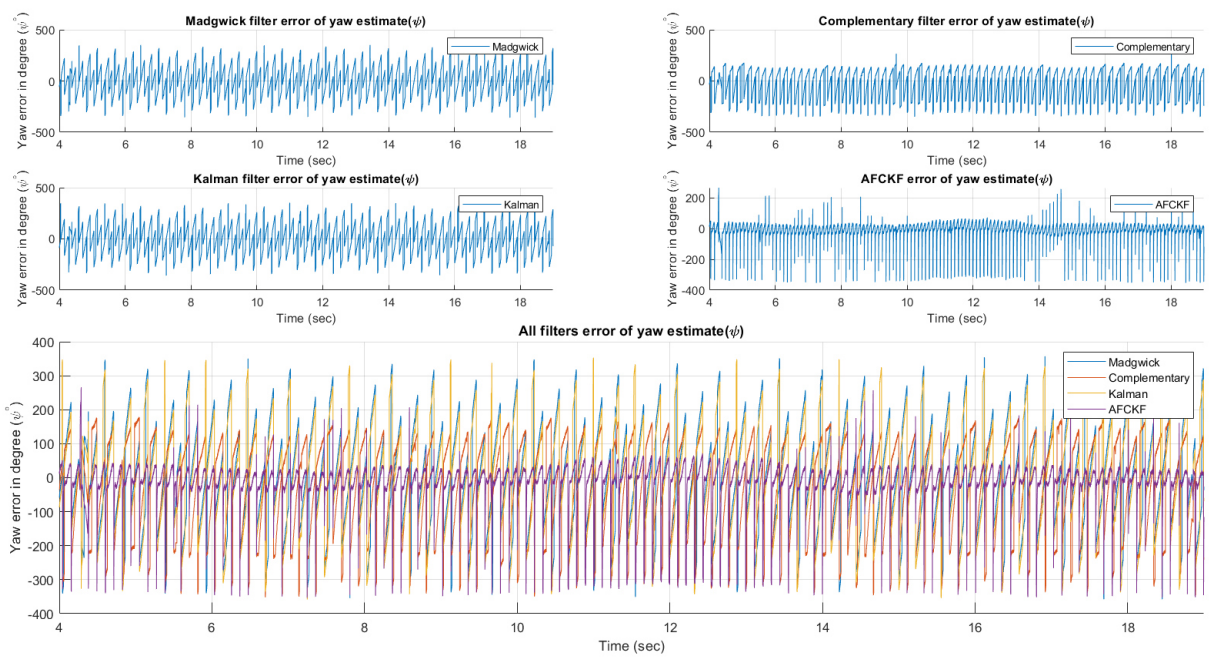

Figure A.46: Yaw estimate error of different filters [Case: Aggressive motion with damaged rotor] 

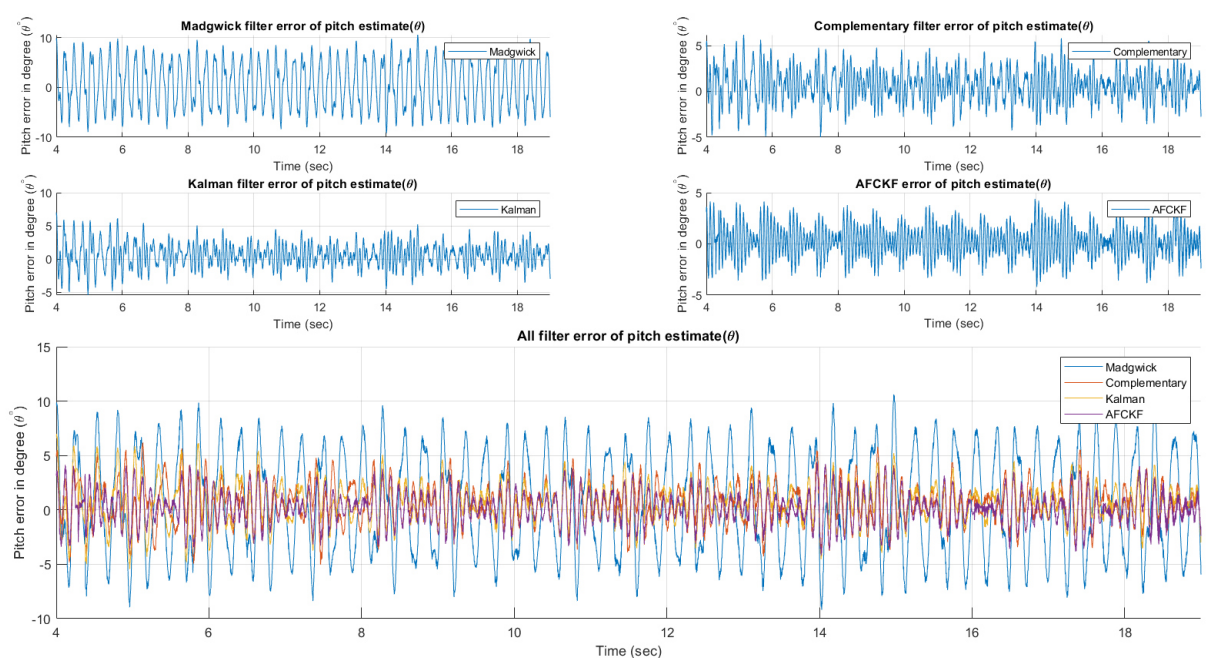

Figure A.47: Pitch estimate error of different filters [Case: Aggressive motion with damaged rotor]
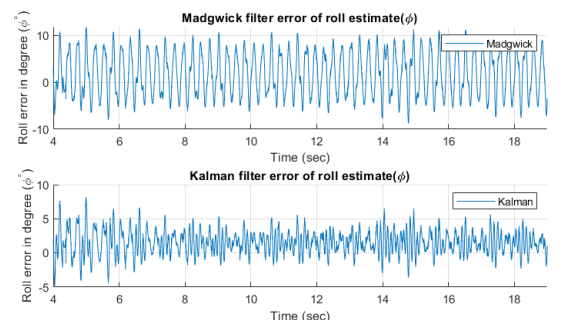
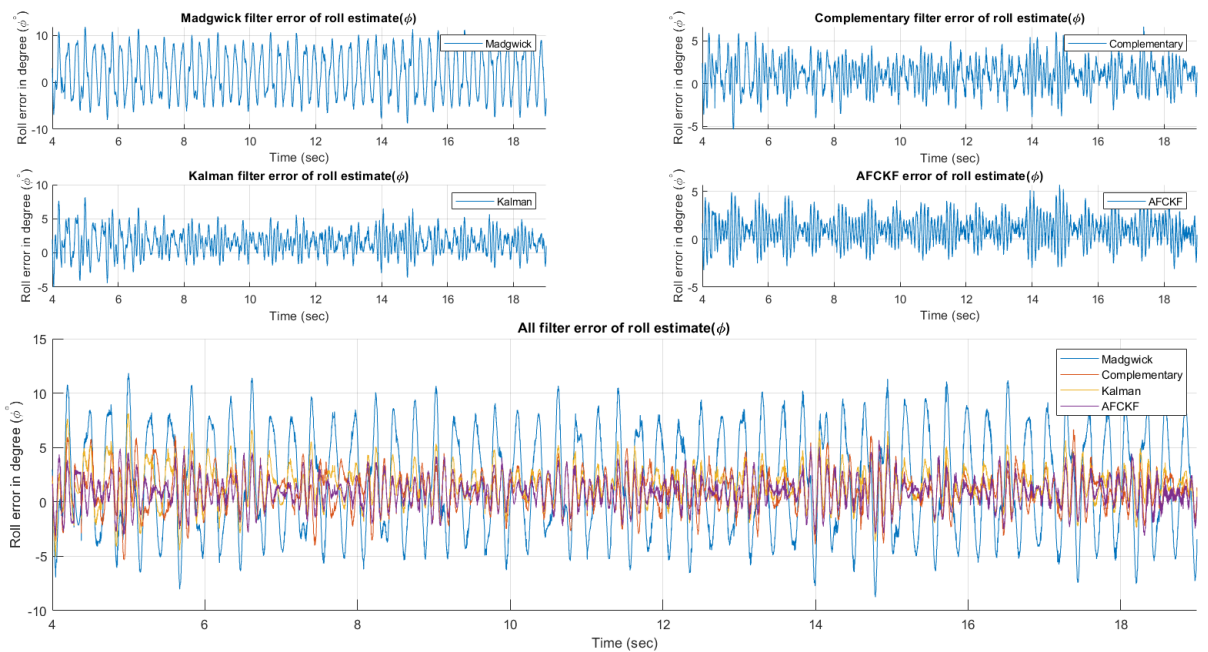

Figure A.48: Roll estimate error of different filters [Case: Aggressive motion with damaged rotor] 


\section{B Determination of the centrifugal model constants}

To determine the value of the centrifugal model constants $\left(\varpi_{1}\right.$ and $\left.\varpi_{2}\right)$, the following approach was adopted: Firstly, experiments were conducted in which the quadcopter was made to rotate about the yaw axis while keeping the roll and pitch of the quadcopter zero or close to zero. The gyroscope and accelerometer readings were collected. Secondly, the accelerometer readings along the $x$ - axis and the $y$-axis were divided by the square of the respective yaw rate gyro readings. Finally, the mean of the obtained value was taken, which resulted in the value of the centrifugal model constants $\left(\varpi_{1}\right.$ and $\left.\varpi_{2}\right)$ :

$$
\left[\begin{array}{l}
\varpi_{1} \\
\varpi_{2}
\end{array}\right]=\frac{\sum_{i} \frac{\left[\begin{array}{l}
a_{x} \\
a_{y}
\end{array}\right]_{i}}{\omega_{z_{i}}^{2}}}{i}
$$

This method was adopted to keep the centrifugal model as simple as possible. The values of the obtained constants are reliable because the accelerometer readings' noise is zero-mean Gaussian distributed and, if the sample size is large enough, the mean equals the real value. 


\section{Comparison of additive and multiplicative versions of the}

\section{AFCKF}

\section{C.1 Case: Hover flight with damaged rotor}

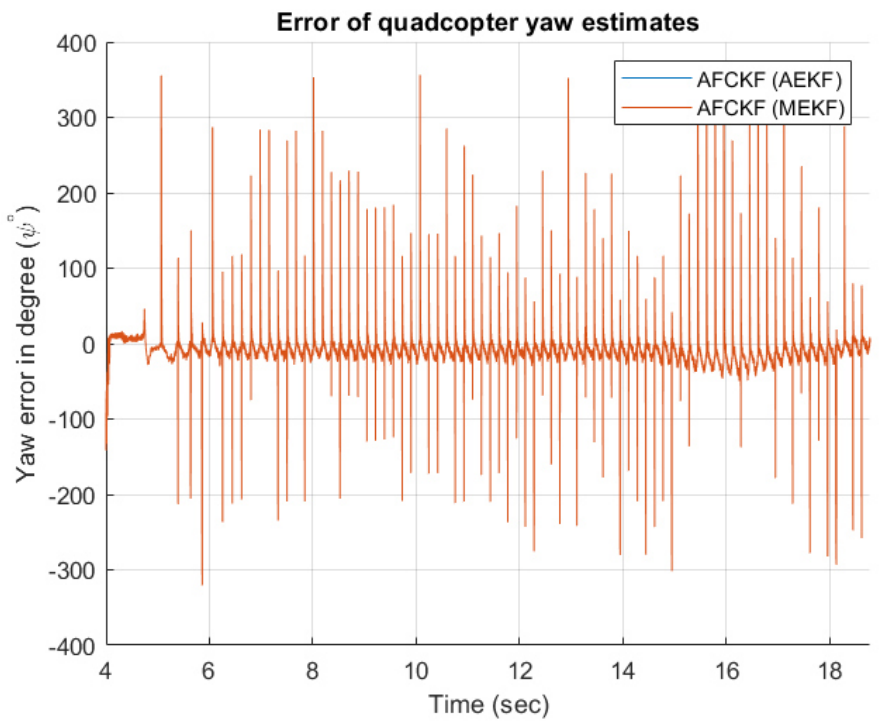

Figure C.1: Yaw estimate error of additive and multiplicative versions of the AFCKF

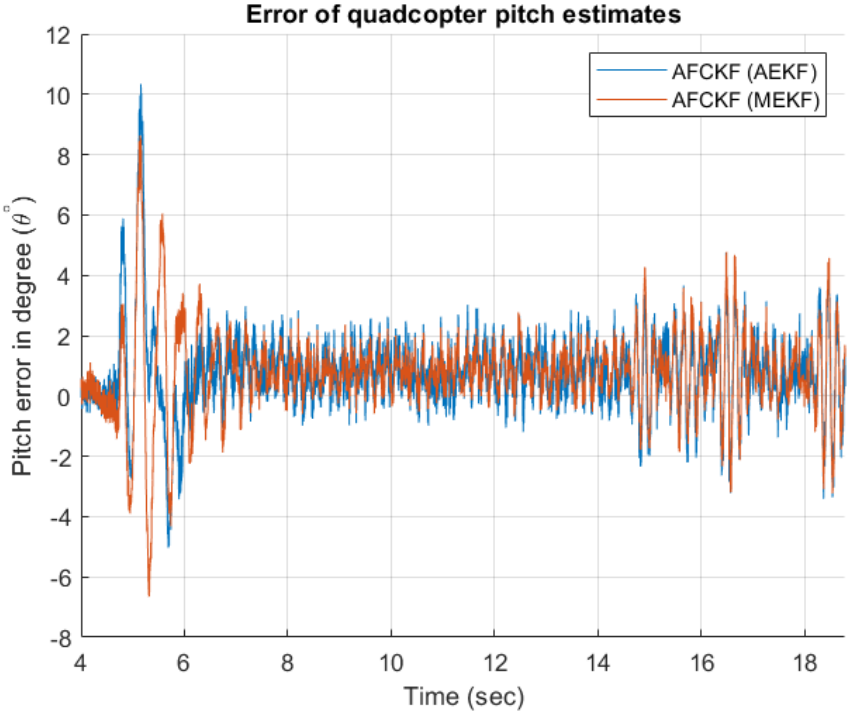

Figure C.2: Pitch estimate error of additive and multiplicative versions of the AFCKF 


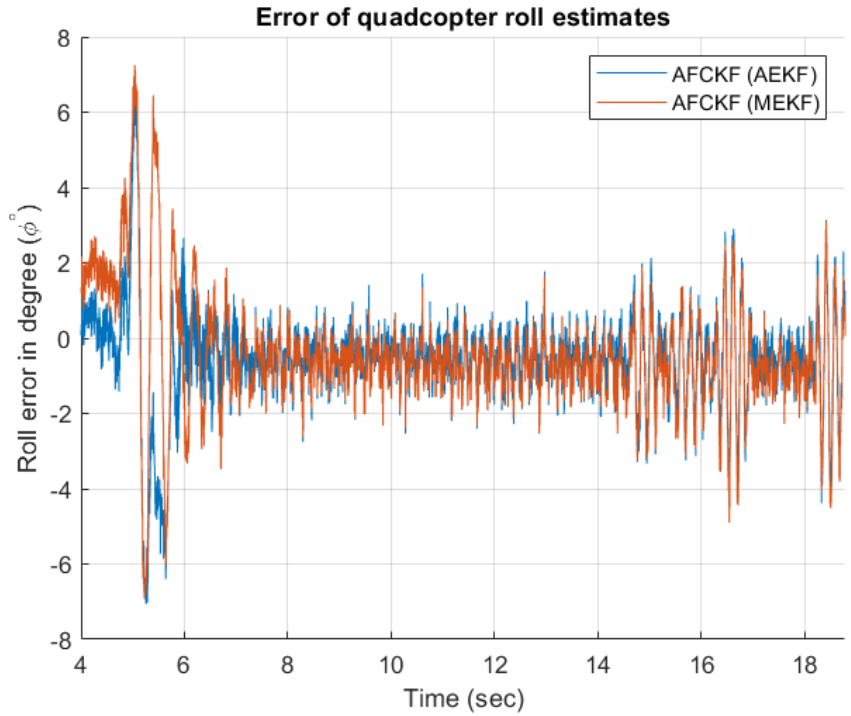

Figure C.3: Roll estimate error of additive and multiplicative versions of the AFCKF

\begin{tabular}{|c|c|c|}
\hline & Additive version & Multiplicative version \\
\hline Yaw $\left(\psi^{\circ}\right)$ & 38.5072 & 38.5072 \\
\hline Pitch $\left(\theta^{\circ}\right)$ & 1.5717 & 1.6108 \\
\hline Roll $\left(\phi^{\circ}\right)$ & 1.4599 & 1.6353 \\
\hline
\end{tabular}

Table C.1: RMS error of additive and multiplicative versions of AFCKF

\begin{tabular}{|c|c|c|}
\hline & Additive version & Multiplicative version \\
\hline Yaw $\left(\psi^{\circ}\right)$ & 1432.8709 & 1432.8709 \\
\hline Pitch $\left(\theta^{\circ}\right)$ & 1.8775 & 2.0200 \\
\hline Roll $\left(\phi^{\circ}\right)$ & 1.7705 & 2.4611 \\
\hline
\end{tabular}

Table C.2: Variance of additive and multiplicative versions of AFCKF 


\section{C.2 Case: Hover flight without damaged rotor}

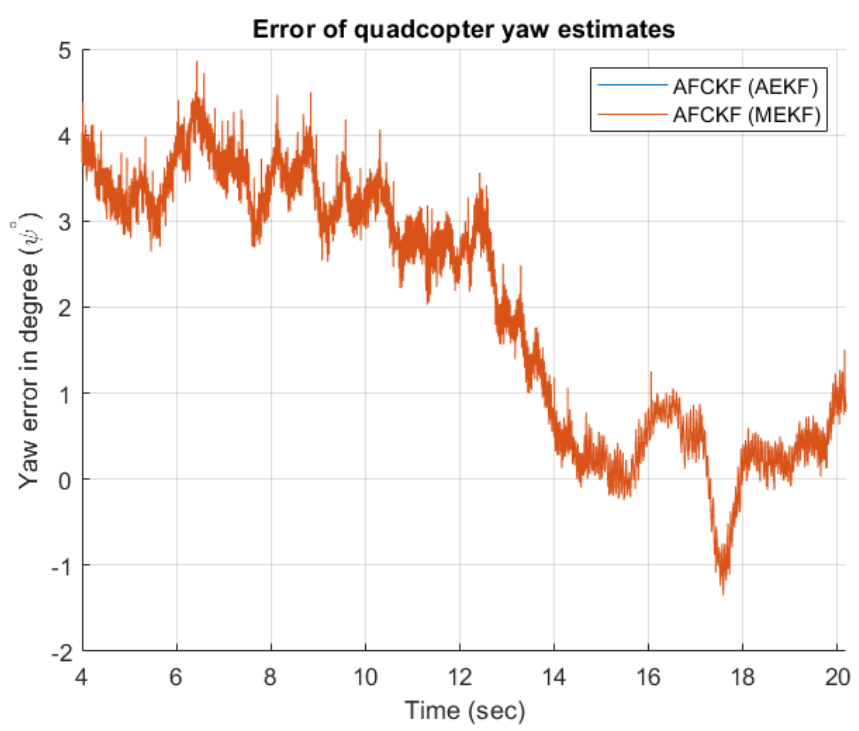

Figure C.4: Yaw estimate error of additive and multiplicative versions of the AFCKF

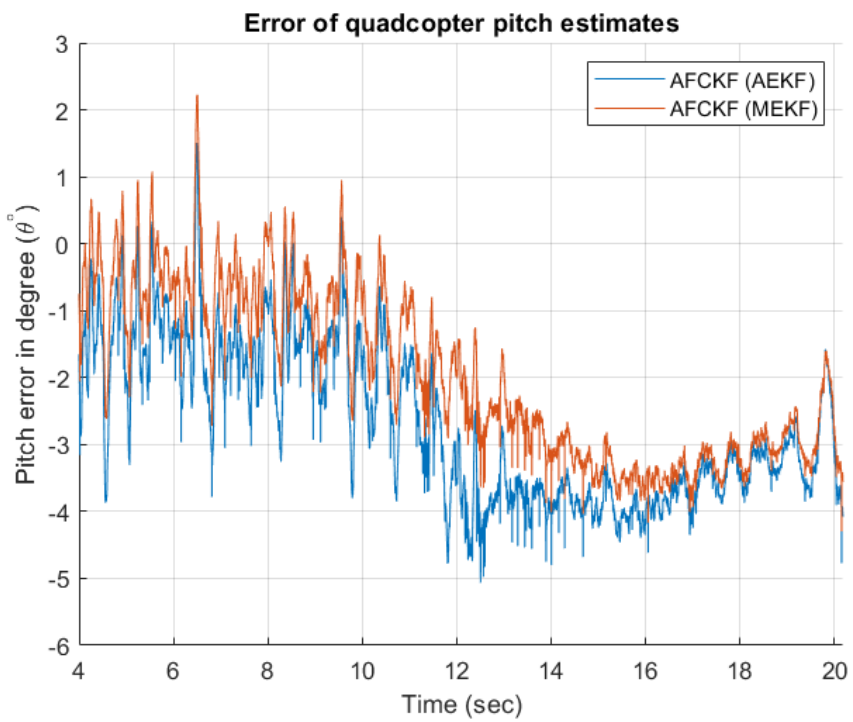

Figure C.5: Pitch estimate error of additive and multiplicative versions of the AFCKF

\begin{tabular}{|c|c|c|}
\hline & Additive version & Multiplicative version \\
\hline Yaw $\left(\psi^{\circ}\right)$ & 2.4921 & 2.4921 \\
\hline Pitch $\left(\theta^{\circ}\right)$ & 2.0445 & 2.0308 \\
\hline Roll $\left(\phi^{\circ}\right)$ & 2.0558 & 1.9986 \\
\hline
\end{tabular}

Table C.3: RMS error of additive and multiplicative versions of AFCKF 


\begin{tabular}{|c|c|c|}
\hline & Additive version & Multiplicative version \\
\hline Yaw $\left(\psi^{\circ}\right)$ & 2.2183 & 2.2183 \\
\hline Pitch $\left(\theta^{\circ}\right)$ & 1.3689 & 1.2039 \\
\hline Roll $\left(\phi^{\circ}\right)$ & 1.1665 & 0.9622 \\
\hline
\end{tabular}

Table C.4: Variance of additive and multiplicative versions of AFCKF

\section{C.3 Case: Aggressive flight with damaged rotor}

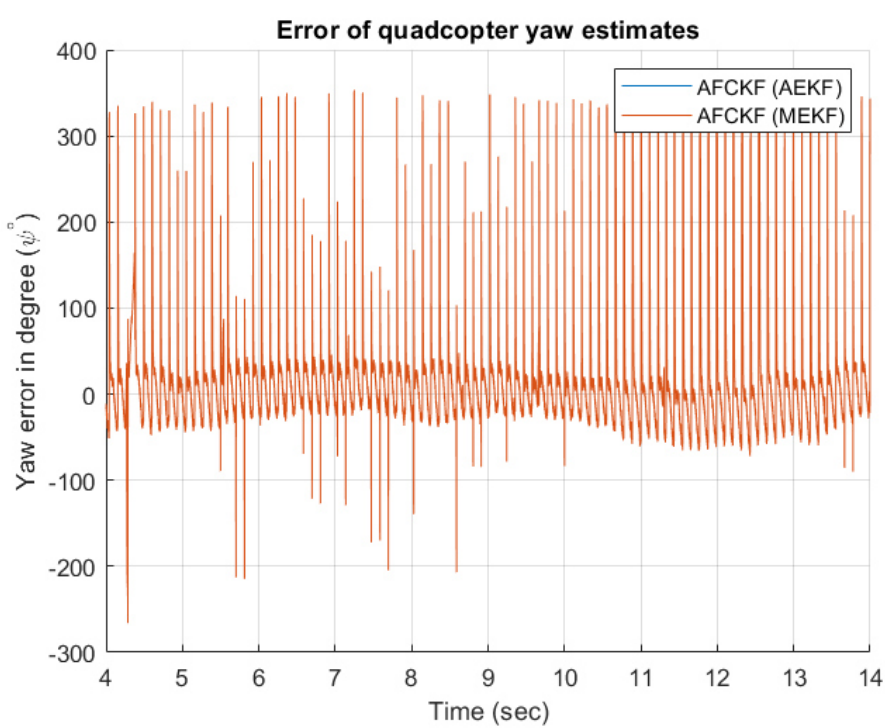

Figure C.6: Yaw estimate error of additive and multiplicative versions of the AFCKF

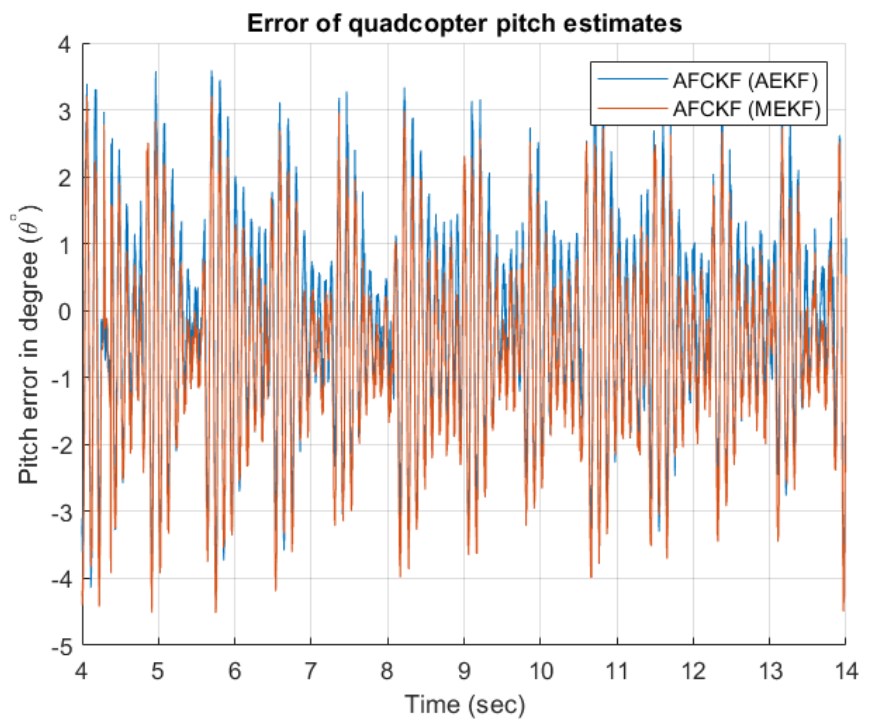

Figure C.7: Pitch estimate error of additive and multiplicative versions of the AFCKF 


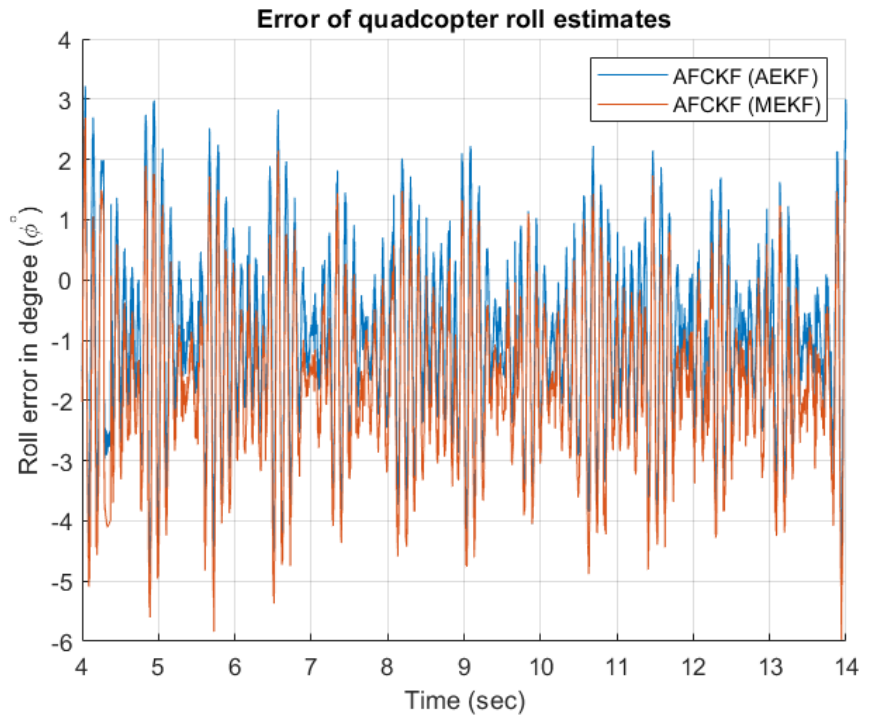

Figure C.8: Roll estimate error of additive and multiplicative versions of the AFCKF

\begin{tabular}{|c|c|c|}
\hline & Additive version & Multiplicative version \\
\hline Yaw $\left(\psi^{\circ}\right)$ & 58.5453 & 58.5453 \\
\hline Pitch $\left(\theta^{\circ}\right)$ & 1.5606 & 1.6223 \\
\hline Roll $\left(\phi^{\circ}\right)$ & 1.7269 & 1.9918 \\
\hline
\end{tabular}

Table C.5: RMS error of additive and multiplicative versions of AFCKF

\begin{tabular}{|c|c|c|}
\hline & Additive version & Multiplicative version \\
\hline Yaw $\left(\psi^{\circ}\right)$ & 3196.6480 & 3196.6480 \\
\hline Pitch $\left(\theta^{\circ}\right)$ & 2.3661 & 2.4245 \\
\hline Roll $\left(\phi^{\circ}\right)$ & 2.1017 & 2.2165 \\
\hline
\end{tabular}

Table C.6: Variance of additive and multiplicative versions of AFCKF 


\section{C.4 Case: Aggressive flight without damaged rotor}

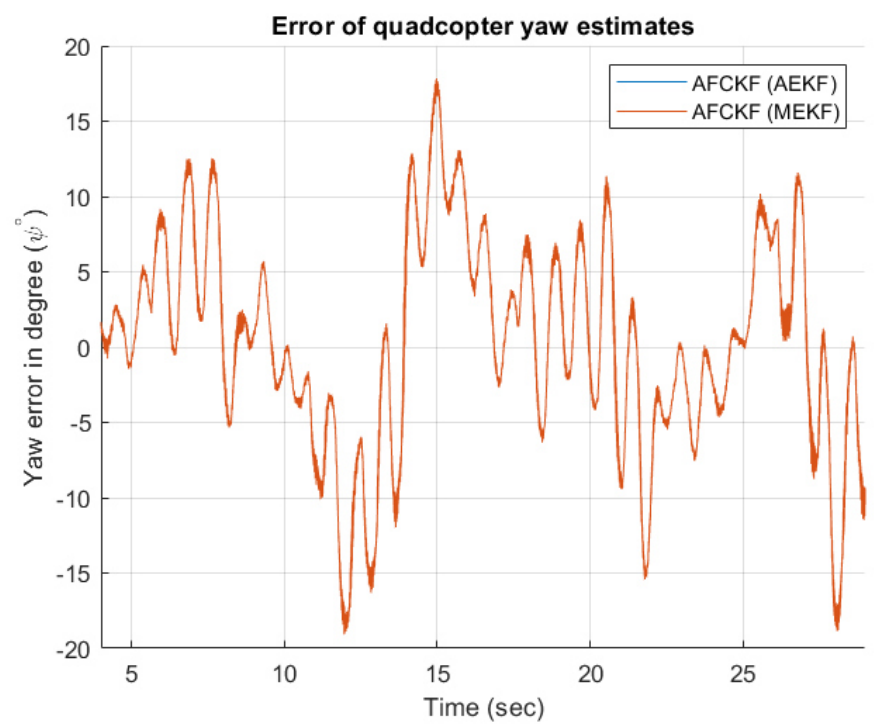

Figure C.9: Yaw estimate error of additive and multiplicative versions of the AFCKF

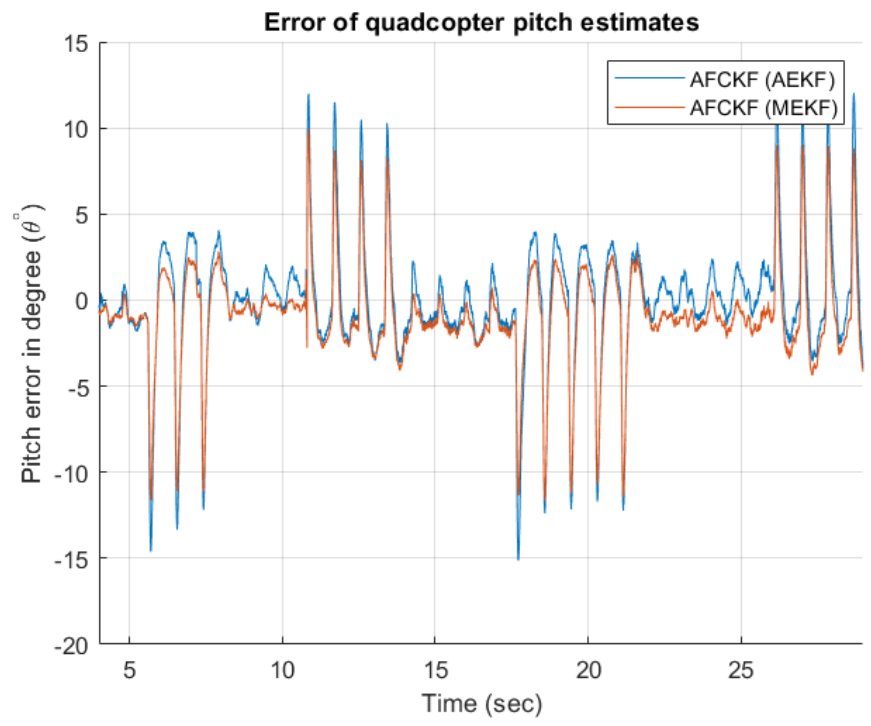

Figure C.10: Pitch estimate error of additive and multiplicative versions of the AFCKF 


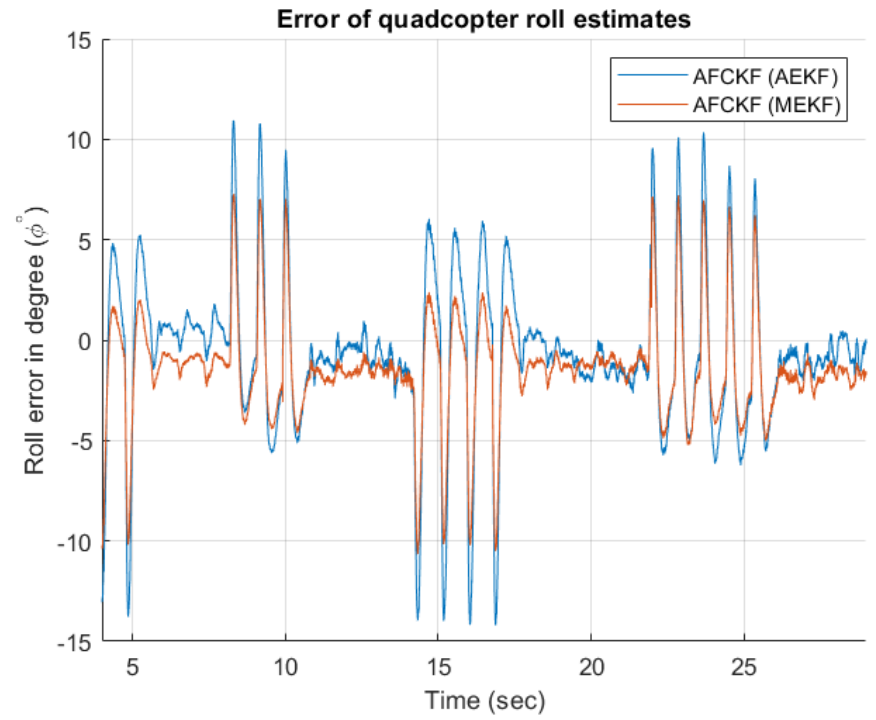

Figure C.11: Roll estimate error of additive and multiplicative versions of the AFCKF

\begin{tabular}{|c|c|c|}
\hline & Additive version & Multiplicative version \\
\hline Yaw $\left(\psi^{\circ}\right)$ & 7.0631 & 7.0219 \\
\hline Pitch $\left(\theta^{\circ}\right)$ & 2.8609 & 2.2267 \\
\hline Roll $\left(\phi^{\circ}\right)$ & 2.9435 & 2.9319 \\
\hline
\end{tabular}

Table C.7: RMS error of additive and multiplicative versions of AFCKF

\begin{tabular}{|c|c|c|}
\hline & Additive version & Multiplicative version \\
\hline Yaw $\left(\psi^{\circ}\right)$ & 49.7615 & 49.7615 \\
\hline Pitch $\left(\theta^{\circ}\right)$ & 11.9753 & 11.3918 \\
\hline Roll $\left(\phi^{\circ}\right)$ & 14.3129 & 14.0119 \\
\hline
\end{tabular}

Table C.8: Variance of additive and multiplicative versions of AFCKF 